\title{
EXAMINING INTERPROFESSIONAL COLLABORATION, PATIENT CARE, STUDENT TRAINING, AND THE EFFECTIVENESS OF INDIVIDUAL COGNITIVE BEHAVIOURAL THERAPY PROVIDED AT AN URBAN FAMILY HEALTH TEAM
}

\author{
by \\ Jennifer R. Rouse \\ Honours Bachelor of Arts, Carleton University, 2008 \\ Master of Arts, Ryerson University, 2011
}

\author{
A dissertation \\ presented to Ryerson University \\ in partial fulfillment of the \\ requirements for the degree of \\ Doctor of Philosophy \\ in the Program of \\ Psychology
}

Toronto, Ontario, Canada, 2016

C) (Jennifer R. Rouse) 2016 


\section{AUTHOR'S DECLARATION FOR ELECTRONIC SUBMISSION OF A DISSERTATION}

I hereby declare that I am the sole author of this dissertation. This is a true copy of the dissertation, including any required final revisions, as accepted by my examiners.

I authorize Ryerson University to lend this dissertation to other institutions or individuals for the purpose of scholarly research

I further authorize Ryerson University to reproduce this dissertation by photocopying or by other means, in total or in part, at the request of other institutions or individuals for the purpose of scholarly research.

I understand that my dissertation may be made electronically available to the public 


\begin{abstract}
Examining Interprofessional Collaboration, Patient Care, Student Training, and the Effectiveness of Individual Cognitive Behavioural Therapy Provided at an Urban Family Health Team
\end{abstract}

Doctor of Philosophy, 2016

Jennifer R. Rouse

Psychology

Ryerson University

Mental illness is highly prevalent in Canada and costs the Canadian economy and health care system billions each year. Yet, Canadians generally do not have access to evidence-based psychotherapy that is considered a frontline treatment in countries such as the United Kingdom and Australia. Family health teams (FHTs) have been proposed as a way to improve access to mental health treatment. Yet, limited research has been conducted on the inclusion of Psychology into FHTs or the training of graduate students in this emerging field. To address this research gap, the novel treatment delivery and student training model at the Ryerson University Psychology Training Clinic (PTC) and St. Michael's Hospital's FHTs was examined in two studies. First, a pilot study examined the effectiveness of individual CBT provided by graduate students to patients with a primary anxiety or depressive disorder. Eighty percent of participants either no longer met diagnostic criteria for their primary mental disorder or were in partial remission. They experienced a significant reduction in symptoms of overall mental health, depression, and anxiety. As well, participants expressed a high level of satisfaction with the services received and reported having a positive working alliance with their student psychotherapist. Results indicate that student-delivered psychotherapy in a FHT setting is an effective treatment delivery model. Second, a two-part mixed methods study was conducted 
evaluating perspectives on patient care, interprofessionalism, and student training through an online study and individual interviews with health care providers, clinical supervisors, graduate students, and patients. Results from this study indicate that the PTC was a positive addition to the FHTs that improved access and provided patients with high quality mental health services. Generally, high levels of interprofessionalism were reported, though some drawbacks and individual differences were noted. The PTC was described as a valuable training experience. The importance of supervision and specific interprofessional and FHT training was highlighted. Findings from these studies represent a worthwhile contribution to the FHT and primary care psychology literature. Furthermore, the inclusion of Psychology and student trainees into a FHT appears to be a successful, viable option to improve access to effective mental health services. 


\section{Acknowledgements}

I am immensely grateful for my time at Ryerson University in the Department of Psychology which has allowed me to grow as a researcher, clinician, and person. The supportive community within the department, including my peers, professors, and support staff, allowed me to pursue projects I am interested in and passion about, including this $\mathrm{PhD}$.

Throughout the past seven years of my MA-PhD, I have been fortunate enough to have two exemplary mentors, my PhD supervisor Dr. Kelly McShane, and fellow dissertation committee member Dr. Diana Brecher. Their integrity, kindness, and insightfulness will continue to guide me throughout my career. I will never be able to fully express my gratitude to you both for your mentorship and support.

This project took the effort of numerous people. I am sincerely grateful to all those who contributed, including considerable support from Dr. Candice Monson. Without the efforts and time of Dr. Kelly Horner and the graduate students' psychotherapists at the PTC, this project would not have been possible. Your contributions were invaluable; I cannot thank you enough. Additional thanks to the health care providers and reception staff at the St. Michael's Hospital for your help and engagement in the project. My most sincere gratitude to all those who participated in this study. Thank you to those who helped in my HLM training. Lastly, I am grateful to Dr. Souraya Sidani and Dr. John Walker for your role on my dissertation committee.

Finally, I would like to thank all of my family, friends, and fellow psychology graduate students for their incredible support over the years. I especially want to thank my husband for his immeasurable support throughout this long process. A special thank you to my son who approved of my time away while I did "The Research." 


\section{Dedication}

This work is dedicated to Sunny (1997-2016) and Miles (2007-2016). Too sad to be apart, they died within months of each other. Their gentle company and constant love will forever be missed. 


\section{Table of Contents}

Author's Declaration for Electronic Submission of a Dissertation...................................ii

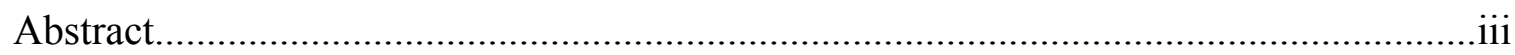

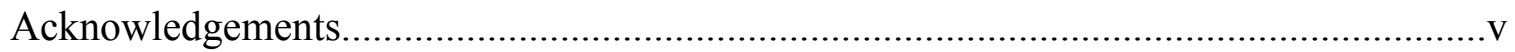

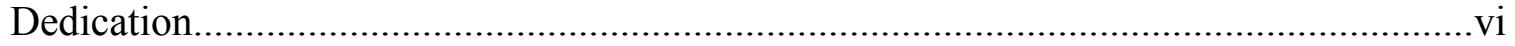

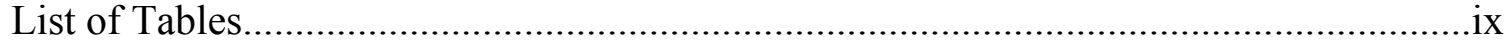

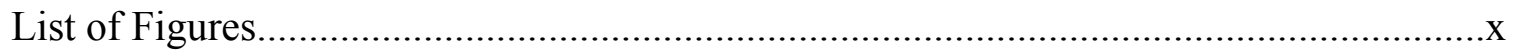

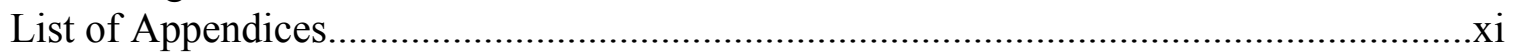

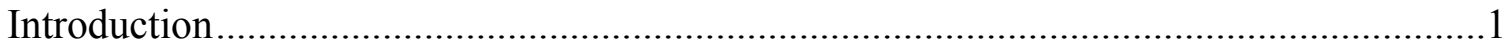

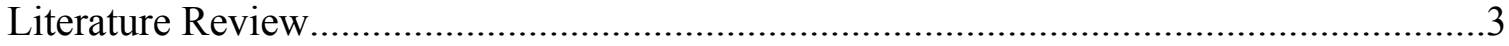

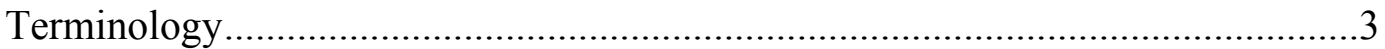

An Overview of Mental Illness in Canada............................................................4

The Effectiveness of Psychotherapy for Mental Disorders ..................................6

Access to Psychotherapy in Canada ....................................................................

Primary Care and Interdisciplinary Family Health Teams ...............................10

Training in Interdisciplinary Primary Care Teams .......................................... 12

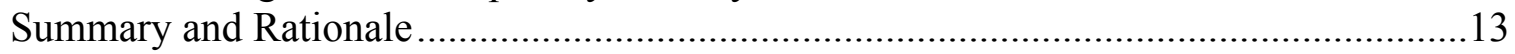

St. Michael's Hospital FHTs and the Ryerson University PTC .........................15

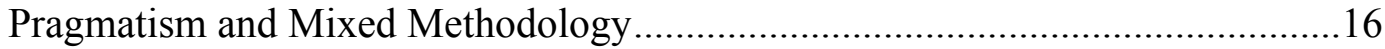

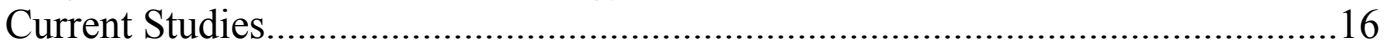

Objectives, Hypotheses, and Research Aims........................................................... 17

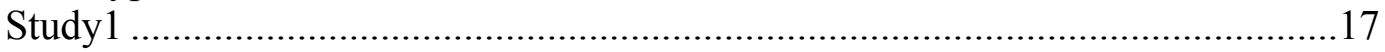

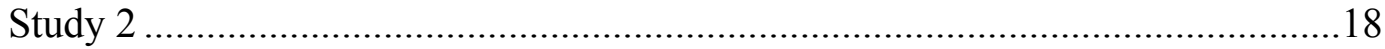

\section{STUDY 1: PSYCHOTHERAPY EFFECTIVENESS PILOT STUDY}

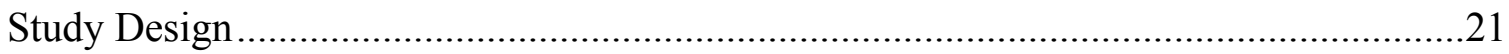

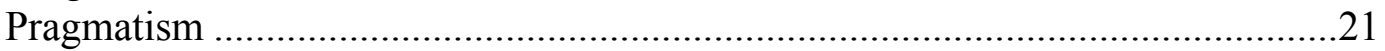

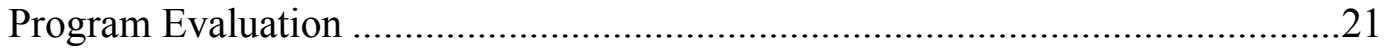

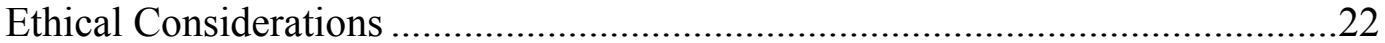

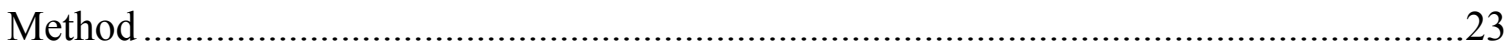

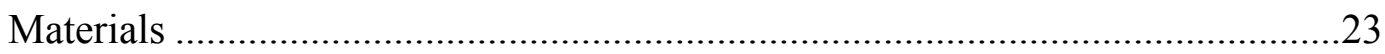

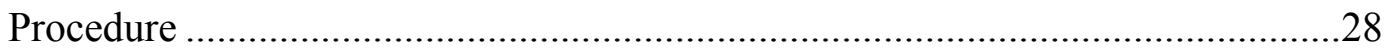

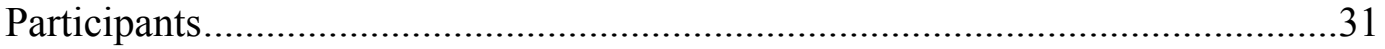

Clinical Psychology Graduate Student Psychotherapists ..................................35

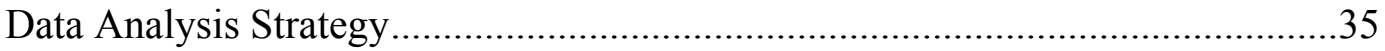

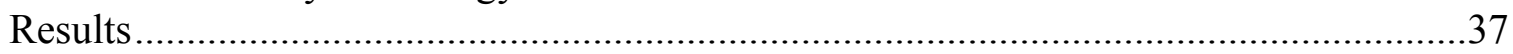

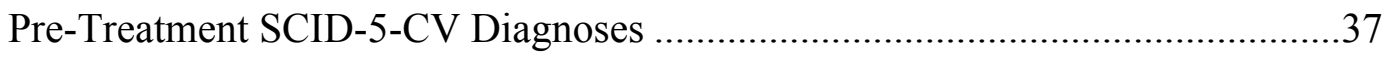

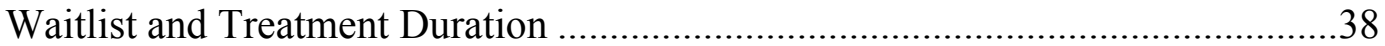

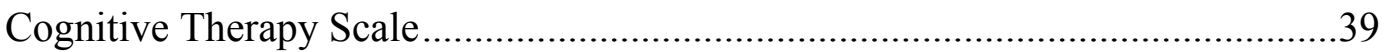

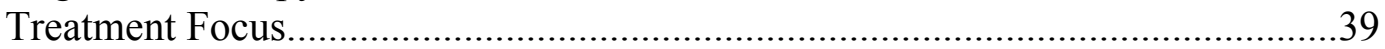

Hypothesis 1: Diagnostics and Symptom Changes .........................................40

Hypothesis 2: Therapeutic Alliance and Satisfaction .....................................67

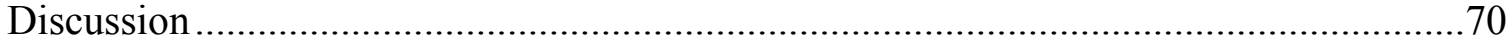

Effectiveness of Individual CBT for Anxiety and Depression ...........................71 
Limitations and Future Directions ......................................................................

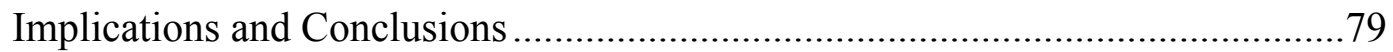

STUDY 2: PERSPECTIVES ON PATIENT CARE, INTERPROFESSIONALISM, AND STUDENT TRAINING AT THE PTC

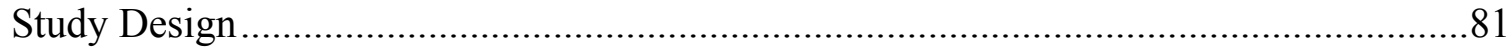

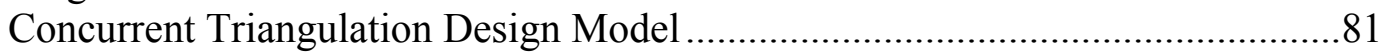

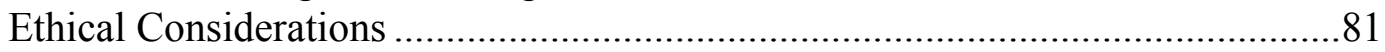

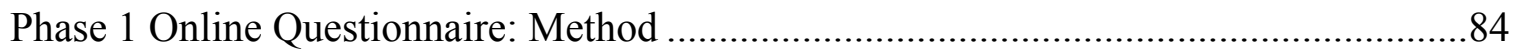

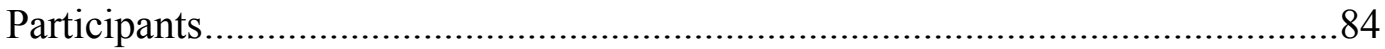

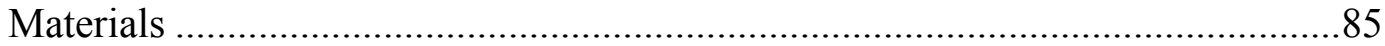

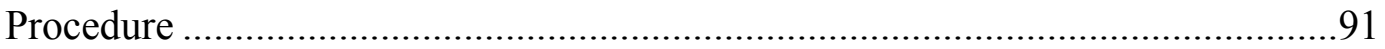

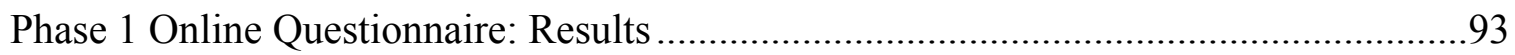

Interprofessional Patient Care ...........................................................................93

Clinical Psychology Graduate Student Training..................................................108

Correlations Between Measures …………………......................................111

Phase 2 Interviews: Methods ……………...................................................112

Participants........................................................................................112

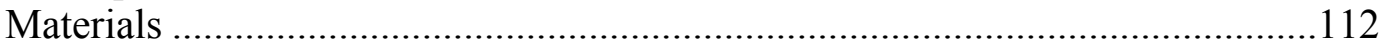

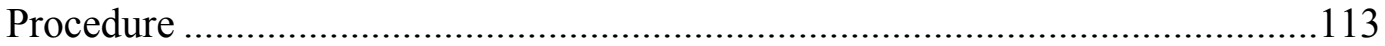

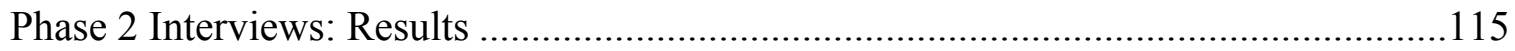

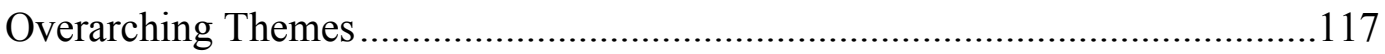

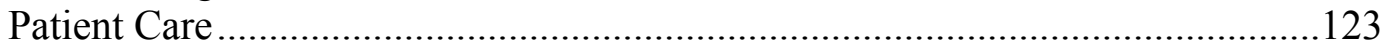

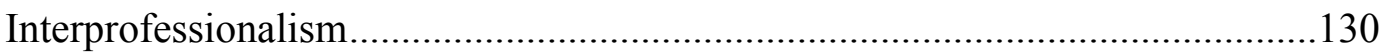

Clinical Psychology Graduate Student Training................................................140

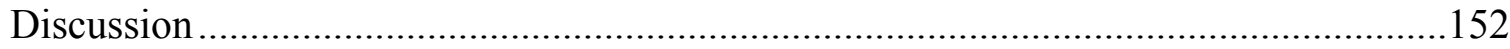

Perspectives on Interprofessional Patient Care and Student Training .................153

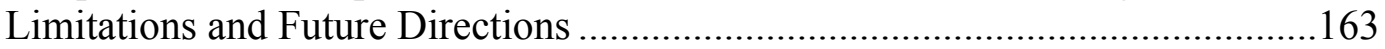

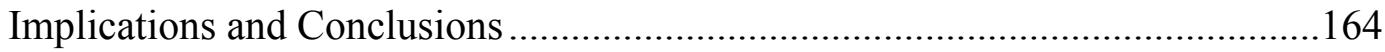

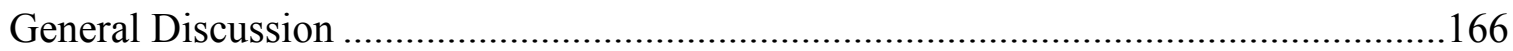

Individual CBT Provided in a FHT and Student Training Clinic .......................167

Perspectives on Patient Care, Interprofessionalism, and Student Training ..........168

Strengths and Limitations ............................................................................171

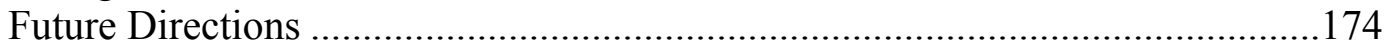

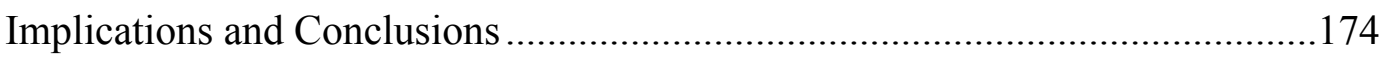

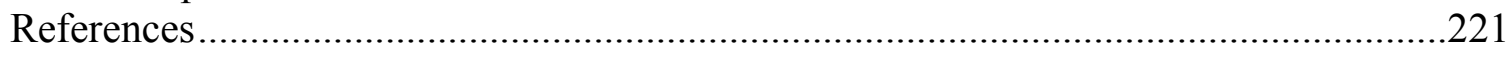




\section{List of Tables}

Table 1. Demographic Information for the Treatment and Intake Only Groups.............. 34

Table 2. SCID-5-CV Diagnoses for Treatment and Intake Only Groups........................ 38

Table 3. Changes in Pre-Post SCID-5-CV Primary Diagnoses Treated........................... 42

Table 4. Medication Use, Social Support, and Working Alliance as Predictors for SCID5-CV Diagnostic Changes........................................................................ 42

Table 5. Pre-Post Treatment OQ-45 Scores in Relation to Clinical Cut-Offs $(\mathrm{N}=31) \ldots \ldots . \quad 44$

Table 6. Descriptive Statistics for HLM for the OQ-45, Session Number, and Predictor Variables.......................................................................................... 45

Table 7. Total OQ-45 Mean Scores by Session...................................................... 46

Table 8. Results of Hierarchical Linear Modeling for the OQ-45 $(\mathrm{N}=31) \ldots \ldots \ldots \ldots \ldots \ldots \ldots \ldots$

Table 9. Frequency of Pre-Post Treatment DASS-21 Scores in Relation to Clinical Cut-

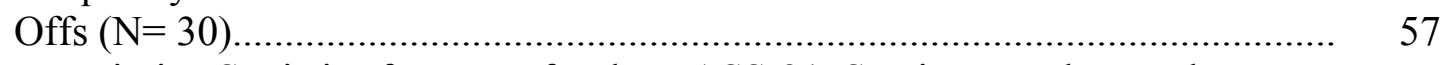

Table 10. Descriptive Statistics for HLM for the DASS-21, Session Number, and Predictor Variables..................................................................................... $\quad 58$

Table 11. Total DASS-21 Mean Scores by Session....................................................... 59

Table 12. Results of Hierarchical Linear Modeling for the DASS-21 $(\mathrm{N}=31) \ldots \ldots \ldots \ldots \ldots \ldots . . . . . . .61$

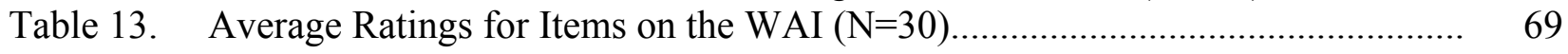

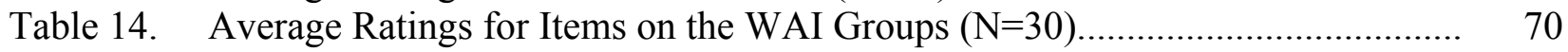

Table 15. Demographic Information for Clinical Psychology Graduate Student, Patient, and Health Care Provider Participants for Online Questionnaire........................ $\quad 85$

Table 16. Comparison of ATHCT Factors and Scores Between Participant Groups.......... 96

Table 17. Comparison of ICS Factors and Scores Between Participant Groups................ 100

Table 18. Health Care Provider's Consultation with Other Team Members: Percentage of Patient Cases, CITM-HCP (N=12)............................................................ 102

Table 19. Clinical Psychology Graduate Students' Consultation with Other Team Members: Percentage of Patient Cases, CITM-HCP (N=25)......................... 103

Table 20. Number of Clinical Psychology Graduate Students and Health Care Providers Who Received and Provided Consultation to Another Health Care Provider at the FHT within the Last Year........................................................................ 105

Table 21. Comparison of IEPS Scores Between Participant Groups (N=32).................. 107

Table 22. Factor Loadings for the Self-efficacy for Interprofessional Experiential

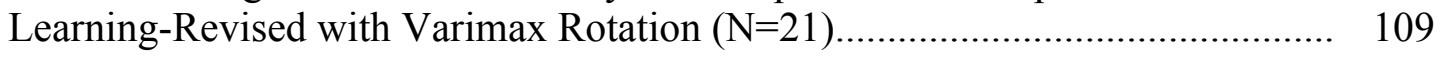

Table 23. Self-Efficacy for Interprofessional Experiential Learning-Revised Scores for

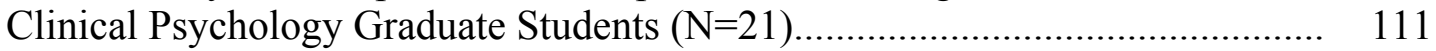

Table 24. Summary of Interview Themes............................................................ 116 


\section{List of Figures}

Figure 1. Effectiveness Pilot Study Patient Flowchart............................................... 33

Figure 2. OQ-45 Total Means by Session Number..................................................... 47

Figure 3. Mean OQ-45 Scores by Session Number.................................................... 49

Figure 4. $\quad$ Participants' Individual OQ-45 Trajectories Over Time................................ 50

Figure 5. $\quad$ OQ-45 Trajectories Over Time For Participants on Waitlist Versus Treatment... 51

Figure 6. OQ-45 Trajectories Over Time For Participants A Primary Depressive.

Disorder Versus Primary Anxiety Disorder.................................................. 52

Figure 7. OQ-45 Trajectories Over Time In Relation To Medication Use........................ 53

Figure 8. OQ-45 Trajectories Over Time In Relation To Working Alliance With......... Graduate Student Psychotherapist...................................................................... 54

Figure 9. OQ-45 Trajectories Over Time In Relation To Social Support......................... 55

Figure 10. DASS-21 Total Means By Session Number.................................................. 60

Figure 11. Mean DASS-21 Scores By Session Number.............................................. 62

Figure 12. Participants' Individual DASS-21 Trajectories Over Time.............................. 62

Figure 13. DASS-21 Trajectories Over Time For Participants With And Without Intake

Data

Figure 14. DASS-21 Trajectories Over Time For Participants A Primary Depressive

Disorder Versus Primary Anxiety Disorder.

Figure 15. DASS-21 Trajectories Over Time In Relation To Medication Use

Figure 16. DASS-21 Trajectories Over Time In Relation To Working Alliance With Graduate Student Psychotherapist.

Figure 17. DASS-21 Trajectories Over Time In Relation To Social Support....................

Figure 18. Interprofessional Collaboration Scale Total And Factor Scores....

Figure 19. Frequency Of Consultation Provided Or Received By Participants As Reported With The Contact With Interprofessional Team Members-Health Care Provider Version. 


\section{List of Appendices}

Appendix A. Informed Consent for Study 1 .............................................................. 179

Appendix B. Clinical Psychology Training Clinic Master Linking Log ........................... 184

Appendix C. Outcome Questionnaire 45.2 (OQ-45)............................................... 187

Appendix D. Depression, Anxiety, and Stress Scales-21 (DASS 21)............................ 188

Appendix E. Client Satisfaction Questionnaire-8 (CSQ-8)........................................... 189

Appendix F. Working Alliance Inventory-Short Form (WAI-S)....................................... 190

Appendix G. Cognitive Therapy Scale...................................................................... 192

Appendix H. Excel Data Abstraction Form for Study 1 ................................................ 196

Appendix I. Psychology Training Clinic Informed Consent................................................... 198

Appendix J. Informed Consent for the Online Questionnaire............................................. 201

Appendix K. Written Debriefing for Study 2 Phases 1 and 2.......................................... 204

Appendix L. Attitudes Toward Health Care Teams Scale (ATHCT) .................................. 206

Appendix M. Interprofessional Collaboration Scale (ICS)................................................ 207

Appendix N. Interdisciplinary Education Perception Scale (IEPS)...................................... 208

Appendix O. Self-Efficacy for Interprofessional Experiential Learning-Revised 209 (SEIEL-R).

Appendix P. Contact with Interprofessional Team Measure - Health Care Provider 211 Version.

Appendix Q. Contact with Interprofessional Team Measure - Patient Version.................... 212

Appendix R. Demographic Questions for Online Questionnaire.......................................... 213

Appendix S. Interview Questions................................................................................ 215

Appendix T. Informed Consent for Individual Interviews................................................. 217 


\section{Introduction}

Mental illness affects one in five Canadians each year and has a yearly billion dollar economic impact in Canada (Lesage et al., 2006; Lim, Jacobs, Ohinmaa, Schopflocher, \& Dewa, 2008; Mental Health Commission of Canada [MHCC], 2012a, 2012b; Smetanin et al., 2011). Individual psychotherapy, particularly cognitive behavioural therapy, is a well-documented effective treatment for a range of mental disorders, including depression, general anxiety, social anxiety, panic, agoraphobia, substance abuse, disordered eating, and post-traumatic stress (Butler, Chapman, Forman, \& Beck, 2006; Chambless \& Hollon, 1998; Chambless \& Ollendick, 2001; Hofmann, Asnaani, Vonk, Sawyer, \& Fang, 2012; Hunsley, 2002; Hunsley, Elliot, \& Therrien, 2014; Myer \& Payne, 2006; United Kingdom Department of Health [UKDH], 2001). Yet, many Canadians do not have access to psychotherapy due to the lack of support in the Canadian Medicare system and the high cost to access it in private practice settings (Hunsley, 2002; MHCC, 2012a; Myer \& Payne, 2006; Vasiliadis, Tempier, Lesage, \& Kates, 2009). One promising mental health care delivery method is interdisciplinary family health teams (FHTs), which may improve access to psychological services, while at the same time reduce costs (Mulvale, Danner, \& Pasic, 2008; Swenson et al., 2008). St. Michael's Hospital and Ryerson University in Toronto developed a novel approach to provide marginalized populations with access to psychotherapy at no direct cost (Rouse, McShane, \& Monson, 2012). With the establishment of the Ryerson University Psychology Training Clinic (PTC), patients of St. Michael's Hospital's six academic FHTs have access to psychological assessment and treatment provided by clinical psychology graduate students from Ryerson University, under the supervision of a team of clinical psychologists. The PTC is the first training clinic in Canada integrated into an interdisciplinary academic FHT environment, and as such, it is important to 
examine the outcomes of this unique student training and mental health treatment delivery model. And although there is research on collaboration between primary care physicians and mental health care providers (e.g. Kates, Crustolo, Farrar, \& Nikolaou, 2002; Kates, 2008; Swenson et al., 2008), there is limited research on the provision of psychotherapy by clinical psychologists in a FHT setting save for a few (e.g. Chomienne at al., 2011; Cordeiro, Foroughe, \& Mastorakos, 2015). In fact, because the FHT model is relatively new, research exploring the impact of FHTs on mental health outcomes in general is limited (Collier, 2011; Cordeiro et al., 2015).

To address this research gap and evaluate this novel mental health care delivery model, a mixed method study of the PTC at St. Michael's Hospital FHTs was conducted. First, the effectiveness of individual cognitive behavioural therapy (CBT) provided at the PTC was examined through a pre-post pilot study assessing changes in diagnoses and psychological symptomatology over time, from intake to waitlist to the end of treatment (Study 1). In particular, this study investigated individual CBT provided by supervised clinical psychology graduate students at the PTC for consenting FHT patients diagnosed with a primary anxiety or depressive disorder, two of the most common presenting disorders in interdisciplinary and primary care settings (Archer et al., 2012; Bland, Newman, \& Orn, 1997; Bray, Frank, McDaniel, \& Heldring, 2003; Chomienne et al., 2011; White, 2008). For Study 2, perspectives on patient care, interprofessionalism, and student training at the PTC were explored using quantitative and qualitative approaches. For Phase 1, health care providers at St. Michael's Hospital FHT and clinical psychology graduate students completed an online questionnaire comprised of a set of standardized and tailored self-report measures on interprofessionalism, including the impact on patient care and students training in the area. For Phase 2, FHT health 
care providers, clinical psychology graduate students, PTC clinical supervisors, and one patient took part in individual interviews exploring attitudes and experiences on patient care, interprofessionalism, and student training at the St. Michael's Hospital FHTs and PTC. Before outlining these studies in full, the relevant literature will be reviewed, starting with a review of terminology.

\section{Literature Review}

\section{Terminology}

Terms for mental health. First, there is a variety of terms related to mental health care (i.e. mental health, mental illness, mental disorder, mental health issues) that although similar in nature, have distinct meanings (Rouse, 2011). The term mental health denotes the general wellbeing, ability to cope, and productivity of a person based on a continuum from unhealthy to healthy (World Health Organization, 2007). Mental disorder is a more specified term that indicates specific disorders as outlined by the Diagnostic and Statistical Manual of Mental Disorders, currently in its fifth revision, such as major depressive disorder or schizophrenia (American Psychiatric Association, 2013). Mental illness can encompass the term mental disorder, as well as include any distress, dysfunction, or impairment that impedes a person's ability to function in one or more area of their life (Canadian Psychiatric Association, 2010;

Mowbray et al., 2006). Finally, the use of mental health issue, concern, or problem speaks more generally to areas of poor mental health (e.g. poor sleep, life adjustment difficulties, stress), but can also suggest mental illness or a mental disorder (Rouse, 2011). This last group of terms is often used more as a catchall term and is frequently used interchangeably with mental illness.

Terms for multi-professional health care teams. Often the terms interdisciplinary, interprofessional, multidisciplinary, and transdisciplinary are used interchangeably, but they 
actually denote different approaches (Hall et al., 2006). First, interdisciplinary means that professionals from a range of disciplines work collaboratively together to provide patient care or undertake a team project with each professional taking on work based on their unique expertise (Hall et al., 2006). This is similar, but different from interprofessional or multidisciplinary teams, which work toward similar goals but complete work independently rather than more collaboratively (Hall et al., 2006). Lastly, transdisciplinary teams work to integrate different professionals at every level, explore and bridge differing theories, knowledge, and skill sets, and may even include input from community members (Hall et al., 2006; Rosenfield, 1992). FHTs by their definition are interdisciplinary in theory. However, the approach that appears to best match the level of collaboration at the FHT where the PTC is housed is interprofessional.

\section{An Overview of Mental Illness in Canada}

In Canada, the yearly prevalence of having a mental disorder is $20 \%$ or 6.7 million Canadians per year (Lesage et al., 2006; MHCC, 2012a, 2012b; Smetanin et al., 2011); the lifetime prevalence is approximately $43 \%$ (MHCC, 2012a, 2012b; Smetanin et al., 2011). This is similar to the United States where approximately $46.6 \%$ of Americans will develop at least one mental disorder in their lifetime (Kessler et al., 2005a). Anxiety and depressive disorders are among the most common disorders in Canada (Vasiliadis, Lesage, Adair, \& Boyer, 2005), the United Kingdom (Jenkins et al., 2003), Australia (Andrews, Hall, Teeson, \& Henderson, 1999), and United States (Kessler et al., 2005a; White, 2008). The economic burden of mental illness in Canada is upwards of $\$ 50$ billion per year (Lim et al., 2008; MHCC, 2012a; Moore, Mao, Zhang, Clarke, \& Laboratory Centre for Disease Control, 1997; Statistics Canada, 2003; Stephens \& Joubert, 2001; Smetanin et al., 2011). The MHCC, which recently developed a national mental health strategy, indicates that in 2011 alone, $\$ 42.3$ billion went toward the delivery of treatment, 
services, and other health care options for individuals suffering from mental illness (MHCC, 2012b). To put this into perspective, $13.4 \%$ of the economic burden of all illnesses in Canada is accounted for by mental and nervous system disorders, while cardiovascular diseases account for 15.2\%, only two percent more (Hunsley, 2002).

Despite the economic burden of mental illness and the fact that close to half of Canadians will experience a mental illness, evidence suggests that Canadians living with mental illness do not typically receive effective treatment or do not receive treatment at all (Kirby \& Keon, 2006; MHCC, 2012a; Ontario Ministry of Health and Long-Term Care [OMHLTC], 2011). In the United States, the wait for individuals actively seeking treatment is upwards of 11 years between the onset symptoms and treatment (Eisenberg, Golberstein, \& Gollust, 2007; Wang, Berglund, Olfson, \& Kessler, 2004; Wang et al., 2005). Moreover, the majority of individuals who meet criteria for a mental disorder do not receive treatment (Kessler et al., 2005b). These trends can be seen in Canada as well; only $33-50 \%$ of Canadian adults and $25 \%$ of children with a mental illness actually undergo treatment (Bartram, 2012; Bland et al., 1997; Statistics Canada, 2003; Vasiliadis et al., 2005). Individuals with severe mental illness are particularly reluctant to seek treatment due to concerns about the effectiveness of treatment, stigma, and symptoms of the disorder itself (Bebbington et al., 2000; Meltzer et al., 2000). Other prominent barriers to treatment in Canada are as follows: challenges accessing treatment, stigma, lack of acceptability of mental illness, limited services available, long wait times, chronic under-funding of services, lack of access to suitable health care professionals, and inconsistent services across provinces and territories (Gagne, 2005; Kirby \& Keon, 2006; Lesage et al., 2006; MHCC, 2009).

However, the climate in Canada surrounding mental illness is changing (Mulvale, Ableson, \& Goering, 2007). This can be noted in the numerous policy papers and government 
documents (Hunsley, 2002; Mulvale et al., 2007), as well as the recent development of the Ontario mental health strategy, Open Minds, Healthy Minds: Ontario's Comprehensive Mental Health and Addictions Strategy (OMHLTC, 2011), and the Canadian Mental Health Strategy developed by the MHCC, Changing Directions, Changing Lives: The Mental Health Strategy for Canada (MHCC, 2012a). Each of these mental health strategies acknowledge the importance of three key areas to improve mental health care access moving forward: 1) increased access to evidence-based strategies, including psychotherapy; 2) increased access to mental health care in primary care settings; and 3) interdisciplinary, collaborative primary care teams (MHCC, 2012a; OMHLTC, 2011). Evidence suggests that mental health care delivered with these three factors in mind is cost-efficient and effectively treats mental illness (Mulvale et al., 2008; Swenson et al., 2008).

\section{The Effectiveness of Psychotherapy for Mental Disorders}

The effectiveness of psychotherapy for a variety of mental disorders, including major depressive disorder, substance abuse, social anxiety disorder, obsessive-compulsive disorder, generalized anxiety disorder, panic disorder, agoraphobia, eating disorders, specific phobias, and post-traumatic stress disorder, has been well-established, particularly for CBT (Butler et al., 2006; Chambless \& Hollon, 1998; Chambless \& Ollendick, 2001; Health Care, 2002; Hofmann et al., 2012; Hunot, Churchill, Silva de Lima, \& Teixeira, 2007; Hunsley, 2002; Hunsley et al., 2014; Myer \& Payne, 2006; Olatunji, Davis, Powers, \& Smits, 2012; Seligman, 1995; Smith \& Glass, 1977; UKDH, 2001). Psychotherapy, predominantly CBT, has been found to be a costeffective treatment with costs equal to or less than those of pharmacotherapy (Chrisholm et al., 2016; Dobson et al., 2008; Hunsley, 2002; Hunsley et al., 2014; Myer \& Payne, 2006; Roberge, Marchand, Reinharz, Marchand, \& Cloutier, 2004). The World Health Organization reports that 
any modest additional cost of psychotherapy to traditional treatment is offset by substantial improvement in the lives of individuals with a diagnosable mental disorder, as well as economic gains related to improved health and productivity (Chrisholm, 2006; Chrisholm et al., 2016). Moreover, these treatments are often effective in a range of different clinical settings and with diverse patient populations (Chambless \& Ollendick, 2001).

With this evidence in mind, the United Kingdom recommends CBT as a first line treatment for a range of mental disorders, including anxiety disorders (generalized anxiety disorder, panic, agoraphobia, social phobia, specific phobia, obsessive-compulsive disorder, and post-traumatic stress disorder), bulimia nervosa, chronic fatigue, pain disorder, and depressive disorders (UKDH, 2001). Australia also recommends evidence-based psychotherapy, the majority of which is CBT-based; citizens can access psychologists for psychotherapy through their primary care physicians and results, thus far, are positive (Fletcher et al., 2009; Moulding et al., 2009). Mental health groups and researchers advocate for improved access to CBT and psychologists in Canada, which may lead to better mental health outcomes and cost less than current pharmacological treatments offered (Cohen \& Peachey, 2014; Hunsley, 2002; Myer \& Payne, 2006; MHCC, 2009). Furthermore, there are patients who prefer psychotherapy over pharmacological interventions; yet, there continues to be a number of barriers to accessing psychotherapy (Mohr et al., 2006).

\section{Access to Psychotherapy in Canada}

By and large, Canadian Medicare does not cover the costs of psychotherapy. As a result, Canadians typically must access psychotherapy through fee-for-service private practices (Hunsley, 2002; MHCC, 2012a; Mulvale \& Bourgeault, 2007; Myer \& Payne, 2006; Vasiliadis et al., 2009). However, there are some exceptions. First, Medicare covers the costs of 
psychotherapy provided by psychiatrists, but due to long-waitlists and short supply of psychiatrists, access to this option is limited (Kates, 2008; MHCC, 2012a; Mulvale \& Bourgeault, 2007). Second, depending on the severity of a symptoms and geographic location, there are services available in some hospitals, community health centres, and twelve mental health centres across Canada, including the Centre of Addiction and Mental Health in Toronto (Closson, 2008; MHCC, 2012a). However, these services are often only accessible for those with severe or persistent mental disorders or symptomatology, leaving those with mild-tomoderate conditions with even more restricted access to psychotherapy (MHCC, 2012a; White, 2008). Post-secondary institutions also often offer psychotherapy, though this is typically an exclusive service to students and unavailable to the general population (Eisenberg et al., 2007; Gallagher, 2010; Storrie et al., 2010). One final option Canadians are eligible to receive at no direct cost is psychotherapy from primary care physicians.

Primary care providers are typically the first point of access for health care treatment and unlike other specialties, they provide patients with comprehensive, continuous, and coordinated care over the course of their lifespan (Bray et al., 2003). In Canada, primary care physicians are the most frequently visited health care provider for mental health treatment (Fournier, LeSage, Toupin, \& Cyr, 1997; Lesage, et al., 2006; Vasiliadis et al., 2009). Primary care physicians are also often the only professional from which Canadians seek treatment for mental illness (Health Canada, 2002a). In fact, the mental disorders category is the most frequently billed category by primary care physicians (Health Canada, 2002b). Put another way, anywhere from 25-33\% of all primary care physician visits are devoted to mental illness, while another $75-80 \%$ of visits for physical ailments include a mental health element (Gunn \& Blout, 2009; Sharp \& Morrell, 1989; Simon, 1992; White 2008). The most common disorders for which patients seek treatment in 
primary care settings are anxiety and depression (Bland et al., 1997; Bray et al., 2003; White, 2008), which are more frequently treated with medication rather than psychotherapy (Roberge et al., 2011; Roberge, Fournier, Menear, \& Duhoux, 2014). The majority of primary care physicians, who traditionally provide pharmacological treatment, also report providing some form of psychotherapy or counselling to their patients for mental health purposes (Grenier, Chomienne, Gaboury, Ritchie, \& Hogg, 2008; Moulding et al., 2009). In fact, one account lists the amount paid to primary care physicians for counselling or psychotherapy was $\$ 356$ million from 2009 to 2010 (Cohen \& Peachey, 2014; Peachey, Hicks, \& Adams, 2013). Yet, many primary care physicians are uncomfortable providing psychotherapy, consider their formal training in psychotherapy to be inadequate, and are dissatisfied with the level of mental health treatment they are able to provide their patients (Clatney, MacDonald, \& Shah, 2008; Grenier et al., 2008). Primary care physicians have indicated that they would like more support from mental health specialists in the diagnosis and treatment of mental illness (Kainz, 2002; Mulvale, 2006; Vickers et al., 2013). In fact, satisfaction with mental health treatment delivery increases when physicians work in collaboration with mental health specialists (Clatney et al., 2008; Vickers et al., 2013).

To address this gap, mental health groups and advocates have urged the Canadian government to fund psychotherapy and integrate clinical psychologists into routine primary health care (Cohen \& Peachey, 2014; MHCC, 2009, 2012a; Peachey et al., 2013; Romanow \& Marchildon, 2003). This message has also been prominent in the news. For example, a recent article in The Globe and Mail directly argued for Medicare to cover the costs of psychotherapy, criticizing the financial barrier to this evidence-based treatment (Anderssen, 2015). Canadian psychologists similarly espoused their frustration that their services are largely inaccessible to 
the general public due to financial barriers, despite having team of professionals who are trained, licensed, and ready to facilitate, create, and research psychological treatments for Canadians (Cohen \& Peachey, 2014).

The delivery of mental health care through interdisciplinary family health teams (FHTs) is a promising new method that may improve access to psychological services and lead to positive patient outcomes, while at the same time reducing costs (Cuijpers, van Straten, van Schaik, \& Andersson, 2009; Linde et al., 2015; Mulvale \& Bourgeault, 2007; Mulvale et al., 2008; Roy-Sannerud et al., 2012; Swenson et al., 2008). This model may also improve support to primary care physicians and utilize resources in an efficient manner (Kates et al., 2002; MHCC, 2012a; Mulvale \& Bourgeault, 2007). Primary care professionals acknowledge the benefits of adding clinical psychologists to primary care teams, noting improved patient outcomes and access to psychotherapy (Bryan et al., 2012; Chomienne et al., 2011; Turcotte, 2005; Vickers et al., 2013). Fortunately, these recommendations are being put into action with sweeping reforms to primary care across Canada, specifically in relation to interdisciplinary, collaborative teams and targets to improve mental health care (Gagné, 2005; Gagné, Dungeon, \& Kates, 2006; Kates, Gagné, \& Whyte, 2008; Moulding et al., 2009).

\section{Primary Care and Interdisciplinary Family Health Teams}

In the last decade, provinces have revolutionized their primary care delivery models with a surge of interdisciplinary, collaborative primary care clinics, many of which have mental health initiatives (Gagné et al., 2006; Kates, 2008; Kates, McPherson-Doe, \& George, 2011a; Mulvale et al., 2007). In Ontario alone, over 200 interdisciplinary family health teams (FHTs) have been established in the last decade (OMHLTC, 2016; Rosser, Colwill, Kasperski, \& Wilson, 2011). FHTs are designed to provide tailored services to meet the needs of their patient population and 
the community in which they are situated (OMHLTC, 2005b; Mulvale et al., 2007; Rosser et al., 2011). A key element of FHTs is the integration of primary care professionals, which moves beyond the traditional solo primary care physician model (Farmanova, Grenier, \& Chomienne, 2013; Rosser et al., 2011). FHTs typically include primary care physicians, nurses and/or nurse practitioners and any combination of allied health professionals, including, but not limited to, pharmacists, social workers, occupational therapists, psychiatrists, dieticians, counsellors, chiropractors, physiotherapists, and psychologists (OMHLTC, 2005b; Soklaridis, Oandasan, \& Kimpton, 2007). Although clinical psychologists may be viewed as valued members of primary care teams, the number of FHTs that include a clinical psychologist in Ontario, particularly fulltime, is limited (Cordeiro et al., 2015; Moulding et al., 2009; Mulvale \& Bourgeaut, 2007). However, with each year, the number of psychologists in primary care in Ontario is expanding, with current estimates between 20 and 30 part-time or full-time psychologists.

There are a number key elements that promote positive interprofessional collaboration between primary care providers and mental health specialists, including the quality of collaborative relationship, frequency of collaboration, availability of specialties, and onsite mental health professionals (Gerdes, Yuen, Wood, \& Frey, 2001). Collocation of psychologists and physicians, in particular, has been shown to increase interprofessional satisfaction (Clatney et al., 2008; Craven \& Bland, 2006; Farrar et al., 2001; Swenson et al., 2008; Vickers et al., 2013; Xyrichis \& Lowton, 2008). As well, a clear division of labour, specific goals, measureable outcomes, interdisciplinary team training, effective communication, and clinical and administrative support are key to successful primary care team cohesiveness, which in turn can lead to better patient outcomes and satisfaction (Grumbach \& Bodenheimer, 2004; Xyrichis \& Lowton, 2008). Research in practical settings re-affirms these recommendations and proffers 
further ones. For example, a recent Ontario study of 14 FHTs identified five main themes relating to collaborative interprofessional team functioning: 1) re-evaluating health care provider traditional roles and areas of practice; 2) having clear management and leadership; 3) sufficient shared time and physical space to allow for communication and relationship building; 4) facilitation of interprofessional activities and initiatives; and 5) patient education on the FHT model (Goldman, Meuser, Rogers, Lawrie, \& Reeves, 2010a). Although there are a number of recommendations for the promotion of positive interdisciplinary team functioning, there is a lack of formalized training programs at academic FHTs (Soklaridis et al., 2007). As well, the bulk of existing interprofessional training is geared toward physicians rather than other health professionals (Soklaridis et al., 2007). Nonetheless there are some general training guidelines for clinical psychologists in interdisciplinary primary care settings.

\section{Training in Interdisciplinary Primary Care Psychology}

As health care shifts toward primary care, it is recommended that clinical psychology programs include more curricula in the area to better prepare students for jobs in this growing field, including clinical experience in community and primary care environments (GarciaShelton \& Vogel, 2002; Linden, Moseley, Erskine, 2005; Pingitore, 1999; Nash, McKay, Vogel, \& Masters, 2012; Schulte et al., 2004; Sladen, 1979). Primary care psychology is a relatively new specialty (Bray, 2004; Frank et al., 2003) and is defined as the delivery of psychological services relating to psychological and overall health, including health promotion and disease prevention for communities, families, and individuals (Bray et al., 2003). The OMHLTC designates the role of FHT clinical psychologists and psychological associates in FHTs to assess, diagnose, treat, and prevent the development of mental disorders and dysfunction in social, familial, physical, intellectual, and emotional domains (OMHLTC, 2005a). To help meet the 
objectives, it is proposed that clinical psychologists be acculturated early on in their training to interdisciplinary functioning and principles of primary care (Talen, Fraser, Cauley, 2005). Training in this area can also improve collaboration and interprofessional team functioning, as well as lead to a greater understanding of and respect for the knowledge and expertise of different disciplines operating within primary care teams (Schulte, Isley, Link, Shealy, \& Winfrey, 2004). In fact, a hallmark of primary care psychology is working in the same physical space as physicians and other health care providers (Garcia-Shelton, 2006), though this is not always the case (Clatney et al., 2008; Goldman et al., 2010a).

Although several training opportunities have become available (Anderson \& Lovejoy, 2000; Bray, 2004; Dobmeyer, Rowan, Etherage, \& Wilson, 2003; Pingitore, 1999; Schulte et al., 2004; Talen et al., 2005; Twilling, Sockell, \& Sommers, 2000), the vast majority of these are in the United States. Few psychologists are trained to work in interdisciplinary primary care settings, and internship and post-doctoral positions are limited (Bray et al., 2003; Grenier et al., 2008; Masters, Stillman, Browning, \& Davis, 2005; Twilling et al., 2000). Moreover, few training programs provide joint, interdisciplinary training with physicians and psychologists, and those that do exist target specialized illnesses or patient populations (Twilling et al., 2000).

\section{Summary and Rationale}

Psychotherapy is an effective treatment for a range of mental disorders, including anxiety and depression (Butler et al., 2006; Chambless \& Hollon, 1998; Chambless \& Ollendick, 2001; Health Care, 2002a; Hunsley, 2002; Hunsley et al., 2014; Myer \& Payne, 2006; Seligman, 1995; Smith \& Glass, 1977; UKDH, 2001), which are two of the most common presenting problems in interdisciplinary primary health care settings (Archer et al., 2012; Bland, Newman, \& Orn, 1997; Bray, Frank, McDaniel, \& Heldring, 2003; White, 2008). However, there are a number of 
barriers to access this treatment in the Canadian health care system, chief among them is the lack of funding of mental health specialists, including psychologists. A new, promising mental health delivery method is interdisciplinary primary care teams that include access to allied health professionals (Mulvale et al., 2008). In Ontario alone, over 200 FHTs have been established in less than 10 years (OMHLTC, 2016). However, this expansive growth has not been matched by research on the impact of FHTs on health and the integration of health care professionals (Collier, 2011; Rosser et al., 2011). While there is research on collaboration between primary care physicians and mental health care providers (e.g. Kates et al., 2002; Kates, 2008; Swenson et al., 2008), there are few studies directly examining the provision of psychotherapy by clinical psychologists in a FHT setting (e.g. Chomienne at al., 2011; Cordeiro et al., 2015). This is due, in part, to the fact that although there are some practicing primary care psychologists in Canada (e.g. Grenier, 2010), psychologists appear to be largely excluded from primary care clinics (Moulding et al., 2009; Mulvale \& Bourgeaut, 2007; Turcotte, 2005). Though, there is some evidence that this is changing with a growing number of psychologists in primary care setting, with as much as 30 part-time or full-time psychologists working in this area in Ontario, alone. Furthermore, there are limited training opportunities in primary care settings (Bray et al., 2003; Grenier et al., 2008; Masters et al., 2005; Twilling et al., 2000) and interdisciplinary academic FHTs (Soklaridis et al., 2007). Moreover, there is limited outcome data or research examining treatment effectiveness, patient satisfaction, or interdisciplinary team functioning and its impact on patient care and mental health (Collier, 2011; Cordeiro et al., 2015; Rosser et al., 2011). St. Michael's Hospital and Ryerson University developed a novel mental health treatment delivery model that addresses many of the challenges. 


\section{St. Michael's Hospital FHTs and the Ryerson University PTC}

St. Michael's Hospital operates six FHT sites, described as Family Practice Clinics through its Department of Community and Family Medicine. These FHTs are located in dense urban centres in central-east downtown Toronto, serving mixed cultural and socioeconomic populations, including homeless persons, students, and urban professionals. In a joint venture with St. Michael's Hospital, Ryerson University opened the PTC at the Health Centre at 80 Bond clinic in 2011, located less than one block south of the psychology research labs at Ryerson. The PTC provides psychological services, including psychological assessment and psychotherapy, solely to patients of the FHTs, seeing approximately 175-200 patients per year. Due to high demand, there is routinely a waitlist for services and between assessment and treatment, similar to other specialties. All psychological services at the PTC are provided by approximately eight to 12 Ryerson University clinical psychology graduate students who are completing a one-year practicum placement. This is a mandatory first practicum placement for all Master's students after their first year of courses, though in the early years of the PTC, PhD level students also completed placements there. Students are supervised by one of three main supervisors who are Ryerson University faculty and licensed clinical psychologists, including the clinical director who is the only full-time Psychology staff at the site. As well, all students receive regular group supervision with the clinical director and an additional clinical supervisor. Patients who receive treatment at the PTC first undergo a psychodiagnostic assessment followed by short-term individual psychotherapy for approximately 12 to 15 sessions, typically CBT. Group psychotherapy has also been offered, sometimes in an interprofessional format, though offerings at the PTC have been less consistently provided over the years. Lastly, in recent years, graduate students have had the opportunity to provide psychoeducational assessments for children. 


\section{Pragmatism and Mixed Methodology}

To address the research gaps presented above and examine the unique treatment delivery and student training model at the joint Ryerson University PTC at St. Michael's Hospital's FHTs, an evaluation comprised of two separate but interconnected studies was undertaken that utilized a pragmatic perspective. This approach allows for the use of a range of available methodologies to assess real world social challenges (Creswell, 2009). It also allows for assessing phenomena 'as it happens,' acknowledging that data is subject to change as time progresses and situations alter (Creswell, 2009; Dures, Rumsey, Morris, \& Gleeson, 2011). Pragmatism takes into account feasibility and applicability (Dures et al., 2011), which are at the heart of the proposed research. A mixed-method evaluation with a concurrent triangulation design was employed. This design denotes that quantitative and qualitative approaches are engaged in simultaneously; results from each study are triangulated to provide a more full understanding of the issues at hand (Baskerville, Hogg, \& Lemelin, 2001; Creswell, Fetters, \& Ivankova, 2004). The concurrent nature of data collection is well suited to and more commonly used in primary care settings due in part because it is less time consuming than other designs (Creswell et al., 2004).

\section{Current Studies}

Study 1 examined the effectiveness of individual psychotherapy provided at the PTC through a pilot study assessing treatment changes in diagnoses and psychological symptomatology over time, by following patients from intake to waitlist to individual treatment. Specifically, this study investigated the effectiveness of individual CBT provided by supervised clinical psychology graduate students at the PTC for consenting FHT patients diagnosed with a primary anxiety or depressive disorder as defined by the Diagnostic and Statistical Manual of 
Mental Disorders, fifth edition (DSM-5; American Psychiatric Association, 2013), two of the most common presenting disorders in interdisciplinary and primary care settings (Archer et al., 2012; Bland et al., 1997; Bray et al., 2003; Chomienne et al., 2011; White, 2008). Study 2 explored perspectives on patient care, interprofessionalism, and student training at the PTC using quantitative and qualitative approaches. For Phase 1 of Study 2, two participant groups, FHT health care providers and clinical psychology graduate students at the PTC, completed an online questionnaire comprised of a set of standardized and tailored self-report measures on interprofessionalism, student interprofessional training, and the impact of interprofessionalism on patient care. For Phase 2 of Study 2, participants from the same groups, as well as one patient and PTC clinical supervisors, took part in individual interviews exploring attitudes and experiences relating to mental health care, collaboration, interprofessionalism, and student training at the St. Michael's Hospital FHTs and PTC.

\section{Objectives, Hypotheses, and Research Aims}

\section{Study 1}

Objective. The objective of Study 1 was to investigate the effectiveness of individual psychotherapy provided by supervised Ryerson University graduate clinical psychology students at the PTC through a naturalistic pilot study to examine whether patients are receiving an effective treatment with which they are satisfied. Although the PTC provides individual psychotherapy for a variety of mental disorders, only individual CBT for a primary anxiety or depressive disorder was examined over time, from patients' intake session to time on the waitlist, and then following them through treatment.

Hypothesis and research aims. It was hypothesized that participants would experience a greater reduction in psychological symptoms as a result of individual CBT as compared to their 
time spent on the waitlist after intake. Specifically, it was hypothesized that:

1. Participants would no longer meet full criteria for a DSM-5 diagnosis of a depressive or anxiety disorder.

2. Participants would experience a reduction in overall symptoms of mental health and symptoms of anxiety or depression and from intake to the end of treatment.

There were also three research aims. First, two factors were evaluated in relation to the treatment patients' received, treatment satisfaction and the quality of the therapeutic alliance. Second, potential predictor variables were examined in relation to study outcomes. These aims are as follows:

1. To evaluate patients' self-reported level of satisfaction with the quality of psychological services received at the PTC.

2. To assess the therapeutic alliance between patients and their clinical psychology graduate student therapists.

3. To examine medication use, working alliance, and social support as potential predictors of change in DSM-5 diagnoses, overall mental health symptoms, and symptoms of depression and anxiety.

\section{Study 2}

Objective. The objective of this two-part study was to generally explore patient and clinician perspectives on and satisfaction with the integration of the PTC into FHT in relation to: 1) interprofessional patient care; 2) interprofessionalism among health care providers and students; and 3) student training at the PTC. To explore these perspectives, a set of online questionnaires (Phase 1) and individual interviews (Phase 2) were completed by: 1) Ryerson University clinical psychology graduate students who received training at the PTC; 2) health care 
providers at the St. Michael's Hospital FHTs, and 3) past or present patients of the PTC. Phase 2 also included feedback from clinical supervisors at the PTC. Guiding this exploratory research were a number of key research questions derived from the research literature and examination of the unique features of this new model of mental health care delivery and student training.

\section{Research questions for Phase 1.}

Interprofessional patient care. What are participants' attitudes toward interprofessional primary care teams? What are participant's perspectives on the impact of interprofessional teams on patient care? What are participants' perceptions of their own profession and the profession of others working at the St. Michael's Hospital FHTs in relation to interprofessional collaboration and training? How much contact do health care providers have with professionals from other disciplines? How satisfied are patients with their care at the PTC? What differences, if any, exist between each participant group with regards to participants' experience with interprofessional patient care? And lastly, what differences exist, if any, between each participant group?

Student training. How confident are participants in the graduate students' ability to engage in interprofessional tasks?

\section{Research questions for Phase 2.}

Patient care. To what extent do physical features facilitate or hinder patient care at the PTC/St. Michael's Hospital FHTs? On the whole, what does the PTC add to the St. Michael's Hospital FHTs in relation to mental healthcare? Are participants satisfied with the quality of patient care provided by clinical psychology graduate students at the PTC? Finally, are there any challenges or drawbacks regarding patient care at the PTC?

Interprofessionalism. To what degree is there respect in communication among the team 
members? Do participants experience a hierarchy of health care professionals versus a teambased collaborative approach? Are there any tensions or challenges arising from this new interdisciplinary treatment delivery model/new clinic? What are participants' experiences of interprofessional communication at the FHTs? What are perceived factors that facilitate or hinder interprofessional team-based functioning? What are participants' understanding of interdisciplinary functioning and professional roles at the St. Michael's Hospital FHTs? And lastly, what differences, if any, exist between each participant group with regards to participants' experience with interprofessional patient care?

Clinical psychology graduate student training. Do clinical psychology graduate students have a good understanding of the FHT model and interprofessionalism? What benefits, challenges, or drawbacks do participants perceive in relation to the training of clinical psychology graduate student the PTC? 


\section{STUDY 1: PSYCHOTHERAPY EFFECTIVENESS PILOT STUDY \\ Study Design}

\section{Pragmatism}

This study used a pragmatic lens that suggests knowledge is gained by engaging in the world and interpreting information as it is available and understanding it within the contexts of a particular situation or in this case, the parameters of a research study (Creswell, 2009; Patton, 2002). Pragmatism is a natural fit with real world clinical research because it recognizes the need to explore practical problems using practical means (Creswell, 2009; Dures et al., 2011). This includes using methods that are achievable in order to examine intended outcomes and develop functional knowledge (Creswell, 2009; Dures et al., 2011; Maxcy, 2003). This perspective acknowledges that the truth is what can be known at the time and is also subject to natural changes that occur over time (Creswell, 2009; Dures et al., 2011). This lens was particularly appropriate given that similar to other clinical settings, there were changes to staff, the referral process, and even a new edition of the Diagnostic and Statistical Manual of Mental Disorders (DSM) throughout the course of the project.

\section{Program Evaluation}

To explore the effectiveness of individual CBT provided at the PTC, a program evaluation was conducted. A program evaluation refers to the examination of a program for quality assurance, quality improvement, and accountability purposes through a range of research methods and measurements (Moritsugu, Wong, \& Duffy, 2010). More specifically, this study employed an outcome evaluation to identify whether the PTC is achieving its intended goals (Moritsugu et al., 2010), namely, the reduction of symptoms of anxiety and depression for patients undergoing individual treatment at the PTC. This program evaluation was framed a 
pilot project due to its size, specific focus (i.e. primary anxiety and depression, CBT), and goal of gathering preliminary evidence on the effectiveness of individual CBT provided at the PTC. Ethical Considerations

Ethical approval and permissions. Prior to commencing this study, approval was obtained from the St. Michael's Hospital Research Ethics Board (received August 8, 2014) and the Ryerson University Research Ethics Board (received August 27, 2014). Where applicable, permission was obtained and fees paid for measures used.

Informed consent. The informed consent provided detailed information about the study, including measures used, the voluntary nature of participation, potential risks and benefits, and contact information of the researchers and Ryerson University and St. Michael's Hospital Research Ethics Boards (see Appendix A). Participants were informed that they could withdraw consent at any time or omit questions without penalty. As well, they were told that only aggregate data would be analyzed. Issues of privacy and confidentiality were outlined, including that data would be kept confidential, anonymized, stored in a secure location, and all electronic data would be encrypted and password protected.

Data storage. All data was kept confidential. Identifying information (e.g. patient names) were initially collected to link patients to their files. Upon completed of the data collection process, participants' were given a participant number to link them to their data. Only aggregate data were analyzed. All data are stored on secure servers, electronic files encrypted and password protected, and all paper documents stored in a locked facility at the Health Centre at 80 Bond, a St. Michael's Hospital's hospital facility. As per Canadian Psychological Association Ethical Guidelines, anonymized data will be kept for 10 years. All informed consents are kept separate from participants' data. Only the researchers and staff associated with 
the PTC (e.g. PTC clinical director) have access to the evaluation data.

\section{Method}

\section{Materials}

\section{Structured Clinical Interview for DSM-5 Disorders, Clinician Version (SCID-5-CV;}

First, Williams, Karg, \& Spitzer, 2015). The SCID-5-CV is a clinician administered standardized semi-structured interview used to assess for DSM-5 diagnoses (First et al., 2015). The measure takes approximately two to four hours to complete.

Psychology Training Clinic Master Linking Log. The PTC Master Linking Log (MLL) is a general clinician administered intake measure to gather information on patients' physical health, mental health, and general demographics. It was adapted from other general medical health questionnaires (e. g. Douglas Williams Executive Program, 2012). The demographic portion of the MLL includes questions about participants' age, gender, religion, cultural background, sexual orientation, relationship status, number of children, highest level of education, and current occupation (see Appendix B). The MLL includes sections related to patients' chronic medical conditions, past mental health diagnoses, prescribed psychotropic medication use, and level of social support. The last section is comprised of a checklist of significant stressors patients may have experienced in the past year, such as relationships with others, finances, physical health, the health of others, housing issues, employment or unemployment, and severe mental illness.

\section{Outcome Questionnaire 45.2 (Lambert, Burlingame, Umphress, Hansen,} Vermeersch, Clouse, \& Yancher, 1996; Lambert, Morton, Hatfield, Harmon, Hamilton, Reid, Shimokawa, Christopherson, \& Burlingame, 2004). The OQ-45 is one of the most commonly used outcome measures world-wide (Hatfield \& Ogles, 2004; Lambert, 2010). 
Decades of research have shown that the OQ-45 is a reliable and internally consistent measure that is sensitive to change and demonstrates strong test-retest reliability (Chapman, 2003;

Lambert et al., 1996, 2004; Miller, Duncan, Brown, Sparks, \& Claud, 2003; Mueller, Lambert, \& Burlingame, 1998; Umphress, Lambert, Smart, Barlow, \& Clouse, 1997; Vermeersch, Lambert, \& Burlingame, 2000; Vermeersch, Whipple, Hawkins, Burchfield, \& Okiishi, 2004).

Comprised of 45 items, the OQ-45 examines overall mental health and functioning using three subscales: Symptom Distress, Interpersonal Relations, and Social Role (Lambert et al., 1996, 2004). Symptoms from common mental disorders, including generalized anxiety disorder, panic disorder, major depressive disorder, and substance abuse, comprise the Symptom Distress scale. It includes statements such as "I feel like something is wrong with my mind," "I have thoughts of ending my life," and "after heavy drinking, I need a drink the next morning to get going." Evidence suggests that relationships with others impacts overall life satisfaction (Lambert, 1996), which is why the OQ-45 measure includes the Interpersonal Relations subscale, comprised of statements such as "I have trouble getting along with friends and close acquaintances" and "I feel unhappy in my marriage/significant relationship." The third subscale, Social Role, relates to work, school, and spare time. Statements for this subscale include "I feel stressed at work/school" and "I enjoy my spare time."

Responses to these items are rated on five-point Likert scale ranging from 0 to 4 , where 0 indicates "Never," 1 indicates "Rarely," 2 indicates "Sometimes," 3 indicates "Frequently," and 4 indicates "Almost Always." Nine of the items are reverse scored. A score of 63 denotes the overall clinical cut-off score; scores below 63 indicate participants' symptoms are more like the general community population, whereas scores above 63 indicate symptoms are more like a clinical population (Lambert et al., 1998, 2004). Clinical cut-offs for the factors are as follows: 
36 for Symptom Distress, 15 for Interpersonal Relations, and 12 for Social Role. Along with clinical cut off scores, the OQ-45 includes reliability cut offs to denote when reliable change has occurred for the total score (reduction of 14 or more) and each of the three factors, Symptom Distress (10 or more), Interpersonal Relations (eight or more), and Social Role (seven or more). The OQ-45 takes approximately seven to 15 minutes to complete (see Appendix C). The OQ-45 demonstrated good internal reliability overall $(\alpha=.95)$, and for subscales Symptom Distress $(\alpha=.92)$, Interpersonal Relations $(\alpha=.84)$, and Social Role $(\alpha=.83)$.

\section{Depression and Anxiety Stress Scale-21 (DASS; Lovibond \& Lovibond, 1995a,}

1995b; Antony, Beiling, Cox, Enns, \& Swinson, 1998). Developed to assess key symptoms of Depression, Anxiety, and Stress (Lovibond \& Lovibond, 1995b), the DASS-21 is a self-report measure that is internally consistent and can accurately distinguish between the three factors, perhaps even more so than the extended 42-item DASS (Antony et al., 1998). Participants are asked to rate the items in relation to the past week using a four-point Likert scale, where 1 indicates "Did not apply to me at all" and 4 indicates "Applied to me very much, most of the time." To gauge symptoms of Depression, the DASS-21 includes seven items such as "I felt that life was meaningless" and "I was unable to become enthusiastic about anything." The Anxiety subscale includes seven items such as "I was worried about situations in which I might panic and make a fool of myself" and "I felt scared without any good reason." The Stress subscale is comprised of seven items, such as "I tended to over-react to situations" and "I found it difficult to relax." For more information, see Appendix D. The DASS-21 total demonstrated excellent internal reliability overall $(\alpha=.95)$, as did the subscales Depression $(\alpha=.94)$, Anxiety $(\alpha=.89)$, and Stress $(\alpha=.88)$. 


\section{Client Satisfaction Questionnaire-8 (CSQ-8; Larsen, Attkisson, Hargreaves, \&}

Nguyen, 1979; Attkisson \& Zwick, 1982; Attkisson \& Greenfield, 2004). The CSQ-8 is an eight item self-report measure often used in psychotherapy research or by organizations that deliver psychotherapy, which has been shown to be both reliable and valid (Attkisson \& Zwick, 1982; Attkisson \& Greenfield, 2004). This brief measure is rated on a four-point Likert scale and includes questions such as, "How satisfied are you with the amount of help you received?," with responses ranging from "Quite dissatisfied" to "Very Satisfied." Half of the questions are reverse coded, such as "Have the services you received helped you to deal more effectively with your problems?" with responses ranging from "Yes, they helped a great deal" to "No, they seemed to make things worse." . The CSQ was determined to be a reliable measure $(\alpha=.94)$. It takes approximately three to five minutes to complete (see Appendix E).

\section{Working Alliance Inventory-Short Form (WAI; Horvath \& Greenberg, 1987, 1989;}

Tracey \& Kokotowitc, 1989). Working alliance is a key element in psychotherapy effectiveness. The WAI examines this construct using three subscales: goal agreement, shared task creation, and the bond between psychotherapist and client, also known as Goal, Task, and Bond (Horvath \& Greenberg, 1987, 1989; Tracey \& Kokotowitc 1989). The WAI is a 12-item self-report measure that includes statements such as, "As a result of these sessions I am clearer as to how I might be able to change" and " and I have established a good understanding of the kind of changes that would be good for me," where the blank stands for the name of the psychotherapist. Responses to statements are rated using a five-point Likert scale where 1 indicates "Seldom," 2 indicates "Sometimes," 3 indicates "Fairly Often," 4 indicates "Very Often," and 5 indicates "Always" (see Appendix F). This measure has been used in a variety of psychotherapy contexts, is highly reliable and valid, and is considered particularly appropriate 
for research studies compared with other measures of therapeutic alliance (Hanson, Curry, \& Bandalos, 2002; Horvath \& Greenberg, 1989; Martin, Garske, \& Davis, 2000; Tracey \& Kokotovic, 1989). It is also considered comparable to the 36 -item version (Busseri \& Tyler, 2003). A reliability analysis for the WAI indicated that it was a reliable measure $(\alpha=.94)$. The subscales were also found to be reliable: Bond $(\alpha=.89)$, Task $(\alpha=.90)$, and Goal $(\alpha=.86)$. Scores can range from 12 to 60 . The WAI takes approximately five to seven minutes to complete.

Cognitive therapy scale (CTS; Young \& Beck, 1980). The cognitive therapy scale is used to measure a psychotherapists' fidelity to CBT treatment (see Appendix G). It is comprised of 13 areas divided into three sections: general therapeutic skills (six items, such as therapists' understanding and interpersonal effectiveness), conceptualization, strategy, and techniques (five items, such as guided discovery and strategy for change), and other considerations (two items, including problems that may have arisen and factors that contributed to therapists' departure from standard practices). Each area is assessed on a scale from 0 to 6 where 0 indicates "Poor" and a score of 6 indicates "Excellent." Scores are accompanied by a description of the level at which the skill was demonstrated. For example, for Agenda, 0 equaled "Therapist did not set agenda" and 6 equaled "Therapist worked with patient to set an appropriate agenda with target problems, suitable for the available time; established priorities and then followed the agenda." The measure is completed for the entirety of the audio or videotaped session being assessed, approximately 45 to 60 minutes. Reliability of this measure is seen as moderate, though this is in line with other fidelity assessment measures and continues to be one of the most commonly used CBT fidelity measures (Rakovshik \& McManus, 2010; Vallis, Shaw, \& Dobson, 1986). The CTS was found to be reliable $(\alpha=.96)$. 
Chart reviews. Chart reviews were conducted in the case of missing data or data verification (e.g. number of sessions, DSM-5 diagnoses).

Excel data abstraction form. To capture changes in patients' outcome measures over time, an excel worksheet was created for clinical psychology graduate students (see Appendix H). This form is embedded with formulae for each measure (i.e. OQ-45, DASS-21, CSQ-8, and WAI) to generate factor and overall scores. There are sections for the patient's name, age, number of sessions, attending clinical psychology graduate student, DSM-5 diagnosis, and treatment manual used, if applicable.

\section{Procedure}

Inclusion criteria. Inclusion criteria were as follows: 1) 18 years of age or older at intake session; 2) primary depressive disorder diagnosis, including Major Depressive Disorder, Pervasive Depressive Disorder, Premenstrual Dysphoric Disorder, Other Specified Depressive Disorder, Unspecified Depressive Disorder, or Bipolar (Major Depressive Episode Primary), or a primary anxiety disorder diagnosis of Panic Disorder, Agoraphobia, Social Anxiety Disorder, Specific Phobia, or Generalized Anxiety Disorder, Obsessive-Compulsive Disorder, or PostTraumatic Stress Disorder; and 3) referred to or receive brief individual CBT for anxiety or depression. There were no exclusion criteria related to comorbid conditions or medication use.

Recruitment and standard PTC processes. Purposefully coinciding with the start of this study, the PTC implemented a new standardized assessment process for all patients at the PTC in order to assess patients' mental disorder diagnoses, general mental health symptomatology, and symptoms of depression and anxiety at intake and throughout treatment. To start, all patients who receive treatment at the PTC must undergo a psychodiagnostic intake assessment for in order to refer patients to the most appropriate services, which may or may not 
be at the PTC. At the first assessment, patients either arrived 30 minutes prior to their intake assessment to complete the PTC informed consent (see Appendix I) and assessment battery of self-report screeners (e.g. DASS-21, OQ-45, WAI, CSQ-8, and other measures not reported in this study) in the waiting room or received these documents via mail to complete and bring to their appointment. Patients met with clinical psychology graduate students who explained reviewed that they were students under the supervision of a registered clinical psychologist and reviewed the PTC informed consent, and the limits of confidentiality. After this, students introduced the program evaluation, including its purpose, requirements of the study, and orally reviewing the study informed consent (e.g. details of the study, the voluntary nature, right to withdraw, and confidentiality). Those who consented to participate in the study signed two copies of the informed consent, one for the patient to keep and one for the program evaluation. This was followed by the SCID-5-CV, administered by a clinical psychology graduate student under the supervision of a registered clinical psychologist. The SCID-5-CV took place over one to two sessions that lasted approximately two hours each, depending on case complexity. Data gathered from these assessments were entered into patients' electronic medical records (as per standard practice), as well as into a patient-specific excel data abstraction form. Data were also abstracted into the Master Linking Log. Patients were then placed on a waitlist for treatment at the PTC and data from consenting patients with a primary diagnosis of an anxiety disorder or depressive disorder were entered into the program evaluation. When a space was available, patients were contacted for individual psychotherapy administered by graduate students, primarily CBT. For the most part, participants completed the OQ-45 and DASS-21 at the first session and every third session thereafter. The CSQ-8 and WAI were also completed at the same time points except for the first session. However, as can happen in real world research, 
questionnaires were not always administered at the proposed time intervals and in some cases were missed altogether. At the final session, patients completed all five measures and were reassessed using the SCID-5-CV for current diagnoses only. In some cases, an extra session was booked for the final SCID-5-CV assessment and/or to complete the final self-report measures. All SCID-5-CV assessments were conducted by a different graduate student than the one who provided individual psychotherapy. Data for this study were extracted from consenting participants who underwent individual CBT for primary anxiety or depression from September 2014 to September 2015.

Treatment drop out. Some participants dropped out of treatment. As dictated by protocol at the PTC, attempts are made by clinical psychology graduate students to re-engage patients in treatment by contacting them by telephone and/or mail. Patients' primary care physicians at the St. Michael's Hospital FHTs are also made aware when patients drop out of treatment via the electronic medical record.

Treatment and intake only groups. As a result of clinical psychology graduate students' placement end date, a number of participants who consented to the program evaluation had yet to start treatment. As such, data are available for two groups: 1) treatment group, participants who received individual psychotherapy, and 2) intake only group, participants for whom only intake data was available by end of the data collection period.

CBT treatment fidelity. Pending patient consent, all psychological services administered by clinical psychology graduate students are videotaped for supervisory purposes. Approximately $10 \%$ of total sessions were scored using the CTS by the principal researcher. Videos were convenience sampled due to hospital security features by which the videos are automatically deleted at regular intervals. 


\section{Participants}

A total of 49 participants were screened into the study. One participant withdrew consent at session one (see Figure 1). There were 17 participants for whom only intake data were available for the following reasons: 1) no treatment as of the end of the data collection period; 2) referred to group treatment, and 3) declined treatment. A total of 31 participants comprised the treatment group, all of whom received at least four individual psychotherapy sessions, yielding at least two treatment data points.

Demographics for all participants are summarized in Table 1 with participants divided the treatment or intake only group. No significant differences were found between groups except for level of education, which was higher in the treatment group where more people completed postsecondary education, $t(45)=2.64, p=.011$, Hedge's $g=0.80$. More women took part in the study $(N=29)$ than men $(N=19)$, which is similar to past CBT effectiveness studies for anxiety and depression (e.g. Hunsley et al., 2013). Participants ranged in age from 18 to 67 ( $M=40.77$; $S D=12.57)$. Most identified as heterosexual $(N=39)$, while six participants identified as homosexual, gay, or lesbian. One participant identified at transgender. The majority of participants were married or in a common law relationship $(N=17)$, single $(N=15)$, or in a longterm relationship $(N=10)$. Only $30 \%$ reported having children. Participants were primarily religious $(55 \% ; N=23)$, though $38 \%$ identified as non-religious. Over half of participants indicated their culture/ethnicity as White $(62 \% ; N=26)$, while the remaining $38 \%$ identified having a range of different cultural or ethnic backgrounds. 
Life stressors. Participants endorsed experiencing a number of life stressors in the past year as of the intake. These included stress related to the following areas: employment/unemployment (73\%), finances (51\%), physical health (38\%), relationship with partner/spouse (34\%), relationship with friends or family (30\%), health of a friend or family member (30\%), severe mental health (15\%), housing (6\%), grief/loss $(6 \%)$, moving $(4 \%)$, and school (4\%). One person each reported stress related to immigration, dating/isolation, and power of attorney difficulties. No significant differences were found between groups.

Social support. The majority of participants indicated that there was someone in their life who provided them with social support (89\%). Two participants (4\%) denied having anyone who provided social support, while three participants $(7 \%)$ noted that they occasionally had someone for social support. No significant differences were found between groups.

Past health. Sixty-seven percent of participants had a previous mental disorder diagnosis prior to receiving services at the PTC. The majority of participants experienced at least one chronic illness (58\%) as well. No significant differences were found between groups.

Medication use. The majority of patients referred to the PTC were currently taking medication for mental health purposes at the time of intake ( $75 \%$ : $N=36)$. No significant differences were found between groups. 


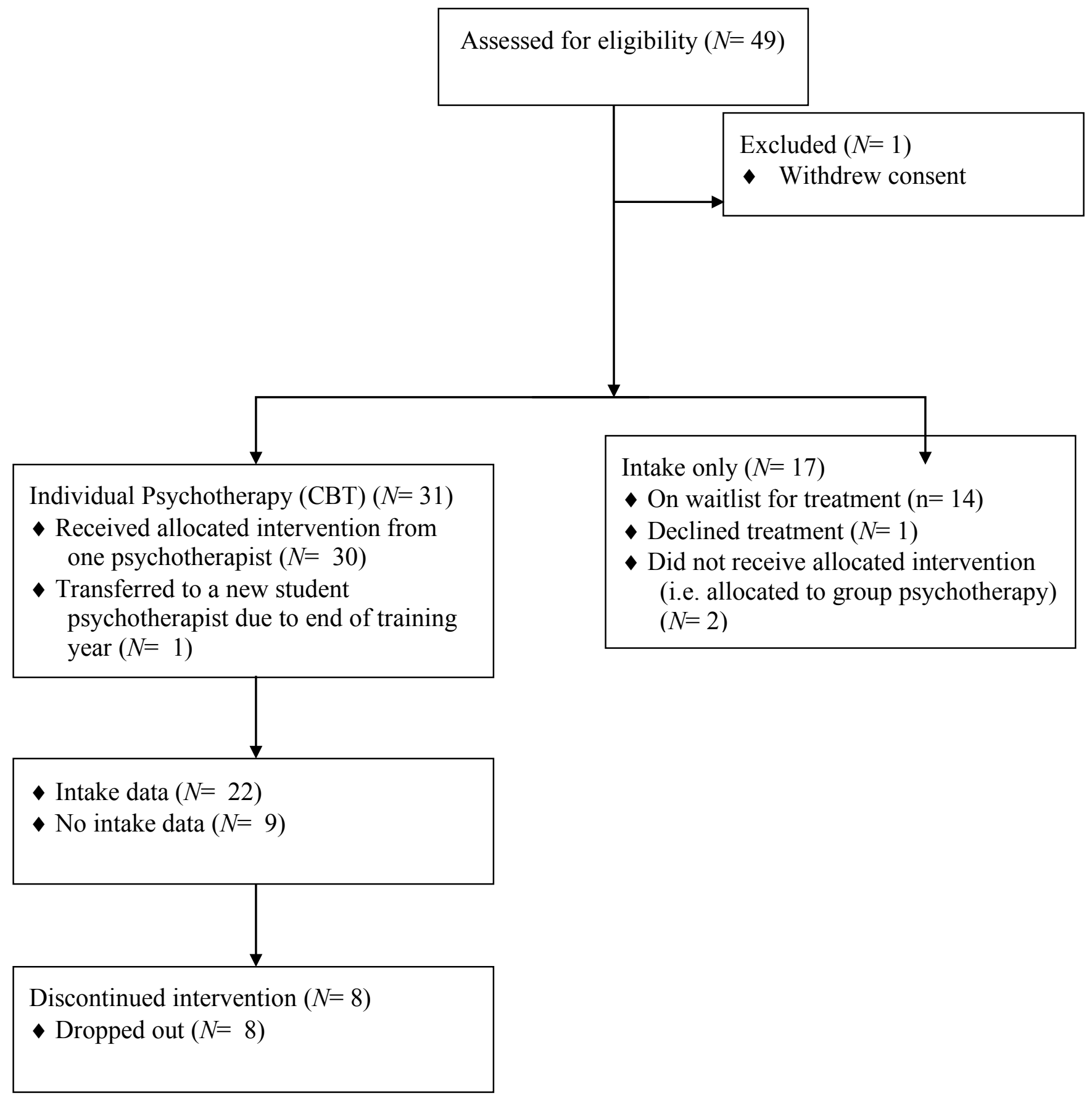

Figure 1. Effectiveness pilot study patient flowchart. 
Table 1

Demographic Information for the Treatment and Intake Only Groups

\begin{tabular}{|c|c|c|}
\hline & $\begin{array}{l}\text { Treatment Group } \\
\qquad \begin{array}{c}(N=31) \\
n(\%)\end{array}\end{array}$ & $\begin{array}{c}\text { Intake Only } \\
\text { Group } \\
(N=17) \\
n(\%)\end{array}$ \\
\hline \multicolumn{3}{|l|}{ Gender } \\
\hline Female & $19(61.29 \%)$ & $10(58.82 \%)$ \\
\hline Male & $12(38.71 \%)$ & $7(41.18 \%)$ \\
\hline \multicolumn{3}{|l|}{ Age } \\
\hline $18-25$ & $3(9.68 \%)$ & $3(17.65 \%)$ \\
\hline $26-30$ & $5(16.13 \%)$ & $3(17.65 \%)$ \\
\hline $31-40$ & $6(19.35 \%)$ & $4(23.53 \%)$ \\
\hline $41-50$ & $8(25.81 \%)$ & $5(29.41 \%)$ \\
\hline $51-60$ & $5(16.13 \%)$ & $1(5.88 \%)$ \\
\hline $61-70$ & $4(12.90 \%)$ & $1(5.88 \%)$ \\
\hline \multicolumn{3}{|l|}{ Relationship Status } \\
\hline Single & $8(25.81 \%)$ & $7(41.18 \%)$ \\
\hline Long-Term Relationship & $6(19.35 \%)$ & $4(23.53 \%)$ \\
\hline Married/Common Law & $11(35.48 \%)$ & $6(35.29 \%)$ \\
\hline Divorced/Separated & $4(12.90 \%)$ & - \\
\hline Widowed & $2(6.25 \%)$ & - \\
\hline \multicolumn{3}{|l|}{ Sexual Orientation } \\
\hline Heterosexual & $29(93.75 \%)$ & $10(66.67 \%)$ \\
\hline Homosexual/Gay/Lesbian & $2(6.45 \%)$ & $4(26.67 \%)$ \\
\hline Transgender & - & $1(6.67 \%)$ \\
\hline \multicolumn{3}{|l|}{ Children } \\
\hline No & $20(64.52 \%)$ & $12(75.00 \%)$ \\
\hline Yes & $11(35.48 \%)$ & $4(25.00 \%)$ \\
\hline \multicolumn{3}{|l|}{ Highest Level of Education Achieved } \\
\hline Some High School & $2(6.67 \%)$ & $3(17.65 \%)$ \\
\hline High School Diploma & $1(3.33 \%)$ & $1(5.88 \%)$ \\
\hline Some College/University & $2(6.67 \%)$ & $6(35.29 \%)$ \\
\hline College/University Degree & $17(56.67 \%)$ & $6(35.29 \%)$ \\
\hline Some Graduate Education/Advanced Certificate or Degree & $1(3.33 \%)$ & - \\
\hline Graduate Education/Advanced Certificate or Degree & $7(23.33 \%)$ & $1(5.88 \%)$ \\
\hline \multicolumn{3}{|l|}{ Religious Affiliation } \\
\hline No & $11(39.29 \%)$ & $8(57.14 \%)$ \\
\hline Yes & $17(60.71 \%)$ & $6(42.86 \%)$ \\
\hline \multicolumn{3}{|l|}{ Cultural/Ethnic Background } \\
\hline Aboriginal & $1(3.33 \%)$ & - \\
\hline Asian & - & $2(20.00 \%)$ \\
\hline Indian/African/West Indies/Caribbean & $2(6.67 \%)$ & - \\
\hline Middle Eastern & $4(13.33 \%)$ & - \\
\hline Spanish/Latin American & $3(10.00 \%)$ & $1(10.00 \%)$ \\
\hline White/European & $20(66.67 \%)$ & $7(70.00 \%)$ \\
\hline
\end{tabular}


Note. Percentage totals may not equal 100 due to rounding. Demographic data is not complete for participants; percentages reflect the available data for each section. No significant differences between groups except for level of education.

\section{Clinical Psychology Graduate Student Psychotherapists}

The PTC practicum takes place from June to May of any given year and includes students completing their first year of the Master's in Clinical Psychology program at Ryerson University. Data collection took place from September 2014 to September 2015, thus spanning two training years. The majority of data collection for this project took place during the September 2014 to May 2015 training year. As a result, 10 of the 11 clinical psychology graduate students who acted as psychotherapists were from the same 2014-2015 training year. There was one graduate student psychotherapist from the June 2015 to May 2016 training year as a result of a client transfer near the end of the data collection period. Pre and post psychological intake assessments were conducted by graduate students from various training years.

\section{Data Analysis Strategy}

Varying analyses were utilized to compare either the intake only and treatment groups, or changes before and after treatment. For continuous data, t-tests were used. For nominal or ordinal data, such as pre-post changes in DSM-5 diagnoses, chi-squared, McNemar, and Wilcoxon Signed Rank tests were used. To examine whether previously documented factors can predict changes in participants' DSM-5 diagnoses as determined using the SCID-5-CV, a binary logistic regression was used. Descriptive statistics were also employed. Effect sizes were calculated where applicable.

Hierarchical linear modeling. There were number of considerations when analyzing the waves of data for the OQ-45 and DASS-21. Both measures were completed repeatedly over time by the same participants, ideally at the same intervals (e.g. intake, session 1, session 4). 
However, as often happens in real-world settings, measures were not always administered at the predetermined intervals. As well, some participants were in treatment longer than others meaning that some participants had more data points than others. Moreover, as hypothesized, social support, medication use, and working alliance must also be taken into account. Therefore, the most appropriate analysis was hierarchical linear modelling (HLM), which can account for all of these issues by tracking waves of data over time. Using a person-period dataset, waves of participant data are tracked over time. HLM provides a more sophisticated statistical model that moves beyond the traditional two data point analysis, thereby providing more detail on the shape of each participants' trajectory of change that is particularly useful for longitudinal studies (Singer \& Willett, 2003). It can also better account for data errors because there are multiple data points, as well as random effects, which are incorporated into the analysis (Singer \& Willett, 2003). Data were available for 31 participants, thereby meeting the threshold of 30 participants or groups needed to run HLM (Kreft \& De Leeuw, 1998; Maas \& Hox, 2005). Restricted maximum likelihood was used due to the study's small sample size (Maas \& Hox, 2005; Raudenbush \& Bryk, 2002). Effect size was calculated using Hedge's $g$, which can be used with HLM and better account for small sample size by using the $t$ distribution (Pustejovsky, Hedges, \& Shadish, 2014).

Missing data. All data were entered into SPSS or excel spreadsheets and carefully checked for errors. Data missing from self-report measures were calculated as indicated in the measures' manual where available, typically an average of available subscale or factor scores. Other missing data, such as descriptive information, number of sessions, and SCID-5-CV diagnoses, were obtained using a chart review of participants' medical records. Missing data imputations were not conducted for missing self-report data at pre-determined assessment 
intervals (e.g. OQ-45 and DASS-21 not administered at session 4). This missing data can be better accounted for by the HLM analysis.

Intention to treat. All analyses for the treatment group are intention to treat analyses. That is, data from participants who dropped out of treatment were retained and not excluded from analyses.

\section{Results}

\section{Pre-Treatment SCID-5-CV Diagnoses}

Pre-treatment SCID-5-CV assessments identified complex diagnoses with four-fifths of participants having two or more DSM-5 diagnoses $(N=38)$ and more than half having three or more diagnoses $(N=25$; see Table 2$)$. Slightly more than half of participants had a primary diagnosis of a depressive disorder (56\%). The remaining half were diagnosed with an anxiety disorder (33\%), obsessive-compulsive disorder $(6 \%)$, or post-traumatic stress disorder $(4 \%)$, of which the latter two were previously classified as anxiety disorders prior to the DSM-5. Secondary diagnoses were predominantly anxiety related $(N=21 ; 57 \%)$ with only five participants who had secondary diagnosis of a depressive disorder (13\%). The remaining third of secondary diagnoses were comprised of complex disorders such as attention deficit hyperactivity disorder, substance use disorder, alcohol use disorder, and anorexia nervosa. Anxiety and depressive disorder remained prominent in tertiary diagnoses $(N=12 ; 48 \%)$, though other mental disorders (e.g. bulimia nervosa, insomnia) were slightly more prevalent $(N=13$; $52 \%)$. No significant differences were found between groups for primary diagnoses $\left(\chi^{2}(11)=\right.$ $11.76, p=.382$, Cramer's $V=0.50)$, secondary diagnoses $\left(\chi^{2}(17)=13.84, p=.678\right.$, Cramer's $V=$ $0.60)$, or tertiary diagnoses $\left(\chi^{2}(14)=15.53, p=.343\right.$, Cramer's $\left.V=0.79\right)$. 
Table 2

SCID-5-CV Diagnoses for Treatment and Intake Only Groups

\begin{tabular}{|c|c|c|c|}
\hline Diagnosis & $\begin{array}{c}\text { Primary } \\
\text { Diagnosis } \\
(N=48) \\
N(\%)\end{array}$ & $\begin{array}{c}\text { Secondary } \\
\text { Diagnosis } \\
(N=37) \\
N(\%)\end{array}$ & $\begin{array}{c}\text { Tertiary } \\
\text { Diagnosis } \\
(N=25) \\
N(\%)\end{array}$ \\
\hline \multicolumn{4}{|l|}{ Depressive Disorder } \\
\hline Major Depressive Disorder & $15(31.25 \%)$ & $4(10.81 \%)$ & $3(12.00 \%)$ \\
\hline Persistent Depressive Disorder & $10(20.83 \%)$ & $1(2.70 \%)$ & $1(4.00 \%)$ \\
\hline Premenstrual Dysphoric Disorder & $1(2.10 \%)$ & - & - \\
\hline Bipolar (Major Depressive Episode Current) & $1(2.10 \%)$ & - & - \\
\hline \multicolumn{4}{|l|}{ Anxiety Disorder } \\
\hline Generalized Anxiety Disorder & $8(16.67 \%)^{1}$ & $3(8.11 \%)^{1}$ & - \\
\hline Social Anxiety Disorder & $6(12.50 \%)$ & $9(24.32 \%)$ & $1(4.00 \%)$ \\
\hline Panic Disorder & $1(2.10 \%)$ & $5(13.51 \%)$ & $1(4.00 \%)$ \\
\hline Specific Phobia & $1(2.10 \%)$ & $2(5.41 \%)$ & $3(12.00 \%)$ \\
\hline Panic Specifier & - & $2(5.41 \%)$ & $3(12.00 \%)$ \\
\hline \multicolumn{4}{|l|}{ Other } \\
\hline Obsessive- Compulsive Disorder & $3(6.25 \%)$ & $1(2.70 \%)$ & $4(16.00 \%)^{1}$ \\
\hline Excoriation (Skin Picking) Disorder & - & $2(5.41 \%)$ & - \\
\hline Illness Anxiety Disorder & - & - & $1(4.00 \%)$ \\
\hline Post-Traumatic Stress Disorder & $2(4.17 \%)$ & $1(2.70 \%)$ & $1(4.00 \%)$ \\
\hline $\begin{array}{l}\text { Other Specified Trauma and Stressor-Related } \\
\text { Disorder }\end{array}$ & - & $1(2.70 \%)$ & - \\
\hline Adjustment Disorder & - & $1(2.70 \%)$ & - \\
\hline Attention Deficit Hyperactivity Disorder & - & $1(2.70 \%)$ & - \\
\hline Substance Use Disorder & - & $2(5.41 \%)$ & $2(8.00 \%)$ \\
\hline Alcohol Use Disorder & - & $1(2.70 \%)$ & $3(12.00 \%)^{1}$ \\
\hline Anorexia Nervosa & - & $1(2.70 \%)$ & - \\
\hline Bulimia Nervosa & - & - & $1(4.00 \%)$ \\
\hline Insomnia & - & - & $1(4.00 \%)$ \\
\hline
\end{tabular}

Note. ${ }^{1}$ Diagnoses include rule out or pre-morbid conditions. Only the first three DSM-5 diagnoses are reported; five participants had four or more diagnoses. No significant differences were found between groups for primary, secondary, or tertiary diagnoses.

\section{Waitlist and Treatment Duration}

On average, participants spent an average of $191.67(S D=54.42)$ days on the waitlist, ranging from 73 days to 310 days. The treatment group spent an average of $189.10(S D=55.74)$ days on the waitlist while those who in the intake only group spent an average of 200.56 days $(S D=51.73)$ on the waitlist. There were no significant differences between groups regarding time spent on the waitlist, $t(38)=-0.55, p=.585$, Hedge's $g=0.21$. Those who received psychotherapy 
spent an average of $103.16(S D=43.42)$ days in treatment.

Sessions. Participants received an average of $10.55(S D=3.69 ;$ Range $=4-16)$

psychotherapy sessions. Those who dropped out of treatment $(N=8)$ received an average of 6.13 $(S D=1.96)$ sessions, while those who remained in treatment $(N=23)$ received an average of 12.09 $(S D=2.78)$ sessions. Not surprising, those who dropped out of treatment received fewer sessions on average than those who remained.

\section{Cognitive Therapy Scale}

Across all participants, 324 psychotherapy sessions took place. Thirty-six sessions were scored using the CTS representing $11.11 \%$ of the all sessions. Videos represented psychotherapy provided by nine of the 11 clinical psychology graduate students $(82.82 \%)$ to 18 of the 31 participants $(58.06 \%)$, ranging from session three to session $15(M=8.94 ; S D=3.48)$. The average CTS score was $57.00(S D=12.85$; Item Mean=4.38), which is above the clinical cut off of 39. The item mean indicates that skill level was "Good" overall. Thus, fidelity to CBT was achieved. Scores ranged from 24 to 72 . Only five of the sessions received a score of 39 or lower, three of which included relationship ruptures and/or non-adherence to treatment. There were no significant differences between patients treated primarily for depression compared to those treated primarily for anxiety, $t(34)=-0.05, p=.958$, Hedge 's $g=0.02$.

\section{Treatment Focus}

Of the 31 participants, the majority were treated primarily for a depressive disorder $(N=11)$ or an anxiety disorder $(N=11)$. Four participants were treated concurrently for depression and anxiety. Two participants received concurrent treatment for depression and another mental disorder (e.g. Alcohol Use Disorder, physical health condition), while one participant received concurrent treatment for an anxiety disorder and another mental disorder 
(e.g. Alcohol Use Disorder, Anorexia Nervosa). One participant primarily received treatment for bereavement in the context of a major depressive episode. Another received primary treatment for Alcohol Use Disorder and concurrent treatment for depression and anxiety.

\section{Hypothesis 1: Diagnostic and Symptom Changes}

It was hypothesized that participants would experience a greater reduction in psychological symptoms as a result of individual CBT as compared to their time spent on the waitlist after intake. Specifically, it was hypothesized that as a result of individual CBT: 1) participants would no longer meet full criteria for a DSM-5 diagnosis of a depressive or anxiety disorder (SCID-5-CV); and 2) participants would experience a reduction in overall mental health symptoms (OQ-45) and symptoms of anxiety or depression (DASS-21) from intake to the end of treatment. It was also hypothesized that previously identified factors might contribute to diagnostic and symptom changes, namely medication use, social support, and working alliance between participants and their graduate student psychotherapist.

Pre-post SCID-5-CV diagnostic changes. Only 20 of the 31participants completed a post treatment SCID-5-CV (current diagnosis being treated only). Of the 11 who did not complete the post treatment SCID-5-CV, eight dropped out of treatment and three declined. Of those who completed both pre and post treatment SCID-5-CV, 16 participants' (80\%) experienced a diagnostic change, either no longer meeting criteria for their primary diagnosis or were in partial remission. Four participants' (20\%) diagnoses remained unchanged (see Table 3). There was a significant difference between groups, $\chi^{2}(1)=7.20, p=.007$, Cramer's $V=0.60$. Medication use, social support, and working alliance have been previously established for accounting for treatment changes. As such a binary logistic regression was conducted to examine the impact of these three factors on pre-post SCID-5-CV diagnostic changes. These 
analyses revealed that these factors did not significantly predict changes in the model (see Table 4). Thus, it appears that medication use, social support, and working alliance did not predict diagnostic changes.

It should be noted that there were limited differences between groups for all of the predictive factors. All of those who did not experience a diagnostic change identified having social support, three of the four were taking medication, and WAI average scores were similar between groups. Moreover, all of those who continued to meet DSM-5 criteria for their primary mental disorder attended between 13 to 14 sessions and did not drop out of treatment. These results indicate that by the end of treatment, the majority of participants no longer fully met criteria for their primary mental disorder, representing a significant change from pre to post treatment that is not predicted by medication use, social support, or working alliance. 
Table 3

Changes in Pre-Post SCID-5-CV Primary Diagnoses Treated

\begin{tabular}{lll}
\hline & \multicolumn{1}{c}{ Pre Treatment } & \multicolumn{1}{c}{ Post Treatment } \\
\cline { 2 - 3 } 1 & \multicolumn{1}{c}{ Major Depressive Disorder } & \multicolumn{1}{c}{ In Full Remission } \\
2 & Major Depressive Disorder & In Full Remission \\
3 & Major Depressive Disorder & In Full Remission \\
4 & Major Depressive Disorder, In Partial Remission & In Full Remission \\
5 & Major Depressive Disorder, In Partial Remission & In Full Remission \\
6 & Major Depressive Disorder and Social Anxiety Disorder & Both in Full Remission \\
7 & Persistent Depressive Disorder and Substance Use Disorder & Both In Full Remission \\
8 & Persistent Depressive Disorder and Generalized Anxiety & Both In Full Remission \\
& Disorder & \\
9 & Bipolar Disorder (Major Depressive Episode Primary) and & Both in Full Remission \\
& Alcohol Use Disorder & \\
10 & Generalized Anxiety Disorder & In Full Remission \\
11 & Generalized Anxiety Disorder & In Full Remission \\
12 & Generalized Anxiety Disorder & In Full Remission \\
13 & Panic Disorder & In Partial Remission \\
14 & Social Anxiety Disorder & In Full Remission \\
15 & Panic Disorder and Rule Out Generalized Anxiety Disorder & Panic Specifier Only \\
16 & Pre-Morbid Obsessive-Compulsive Disorder and Pre-Morbid & No GAD or OCD \\
& GAD & \\
\cline { 2 - 3 } 17 & Specific Phobia Nocial Anxiety Disorder with Panic Specifier & \\
\cline { 2 - 3 } 18 & Social & \\
20 & Pajor Depressive Disorder, In Full Remission & Still Met Full Criteria \\
\hline
\end{tabular}

Table 4

Medication Use, Social Support, and Working Alliance as Predictors for SCID-5-CV Diagnostic Changes

\begin{tabular}{lcccccc}
\hline Predictor & $\beta$ & $\begin{array}{c}\text { Standard } \\
\text { Error }\end{array}$ & $\begin{array}{c}\text { Wald's } \\
\chi^{2}\end{array}$ & $d f$ & $p$ & Exp $(\beta)$ \\
\hline Medication Use & 0.85 & 1.26 & 0.45 & 1 & .501 & 2.33 \\
Social Support & -20.02 & 40193.00 & 0.00 & 1 & 1.00 & 0.00 \\
WAI Average & 0.07 & 0.08 & 0.70 & 1 & .404 & 1.07 \\
\hline
\end{tabular}

Note. WAI = Working Alliance Inventory-Short Form. All analyses were non-significant. 
OQ-45 clinical cut-offs and reliable change. Differences in participants' pre-post total OQ-45 scores ranged from decreasing by 64 to increasing by $17(M=-13.90 ; S D=18.80)$. Prior to treatment, eight participants' total OQ-45 score fell below the clinical cut-off, indicating that their scores were more like those found in a community sample. The remaining 23 participants' scores were above the cut-off, indicating their scores were similar to a clinical sample. At posttreatment, 16 participants experienced a reliable change and nine of those fell below the cut-off, resulting in a total of 17 participants whose scores fell below the clinical cut-off. A related samples McNemar change test showed that this was a statistically significant change from pre to post treatment (see Table 5). It should be noted that seven participants' scores increased by the end of treatment, four of whom dropped out of treatment.

For the Symptom Distress factor, scores from pre to post ranged from a decrease of 35 points to an increase of 10 points $(M=-9.13 ; S D=10.89)$ and 16 participants experienced reliable change from pre to post. Nine of those participants who experienced reliable change shifted from above to below the cut-off, resulting in a total of 17 participants below the cut-off at posttreatment, which was statistically significant. Regarding Interpersonal Relations, six participants experienced reliable change, all of whom had scores shift from above to below the cut-off at post treatment (Range $=-19$ to $6 ; M=-2.81 ; S D=5.19$ ). The least amount of change occurred with the Social Role factor. Only five participants' scores changed from above to below the cut-off and did not represent a statistically significant change (Range $=-14$ to $11 ; M=-1.97 ; S D=4.98$ ). 
Table 5

\begin{tabular}{cccccc} 
Pre-Post Treatment OQ-45 Scores in Relation to Clinical Cut-Offs $(N=31)$ & \\
\hline Above & Below & McNemar & $d f$ & $p$ & Cramer's
\end{tabular}

\begin{tabular}{|c|c|c|c|c|c|c|}
\hline Scale & Cut-Off & Cut-Off & $\left(\chi^{2}\right)$ & & & $V$ \\
\hline OQ-45 Total & & & 7.11 & 1 & $.004 * *$ & 0.48 \\
\hline Pre & 23 & 8 & & & & \\
\hline Post & 14 & 17 & & & & \\
\hline Symptom & & & 7.11 & 1 & $.004 * *$ & 0.48 \\
\hline \multicolumn{7}{|l|}{ Distress } \\
\hline Pre & 23 & 8 & & & & \\
\hline Post & 14 & 17 & & & & \\
\hline Interpersonal & & & 4.17 & 1 & $.041 *$ & 0.37 \\
\hline \multicolumn{7}{|l|}{ Relations } \\
\hline Pre & 20 & 11 & & & & \\
\hline Post & 14 & 17 & & & & \\
\hline Social Role & & & 1.78 & 1 & .180 & 0.24 \\
\hline Pre & 22 & 9 & & & & \\
\hline Post & 17 & 14 & & & & \\
\hline
\end{tabular}

Distress $=36$, Interpersonal Relations $=15$, and Social Role $=12$.

OQ-45 HLM. It was hypothesized that participants would experience a significant reduction in overall mental health symptoms as measured by the OQ-45 over the course of individual CBT. OQ-45 scores (level-1 variable) were explored, taking into account participants' medication use, social support, and working alliance with their graduate student psychotherapist (level-2 predictor variables). For those with both intake and session 1 data, differences in scores while participants were on the waitlist were examined with scores while participants were in treatment using a piecewise HLM analysis. These two time intervals were represented by Time 1 (intake versus session 1) and Time 2 (session 1 to last session). Lastly, differences in OQ-45 scores for those with a primary depressive disorder versus anxiety disorder were explored (level-2 variable). Session numbers acted as the time component, with intake acting as Time 0 (uncentred). Descriptive statistics for HLM can be found in Table 6. 
Table 6

Descriptive Statistics for HLM for the OQ-45, Session Number, and Predictor Variables

\begin{tabular}{lcccc}
\hline Variable & $N$ & $M$ & \multicolumn{1}{c}{$S D$} & Range \\
\hline \multirow{3}{*}{ Sessions } & \multicolumn{3}{c}{ Level-1 } \\
OQ-45 Total & 155 & 5.00 & 4.61 & $0-16$ \\
Intake versus Session1 & 141 & 66.96 & 25.22 & $16-136$ \\
Session 1 versus Last Session & 155 & 0.80 & 0.40 & $0-1^{1}$ \\
& 155 & 4.19 & 4.39 & $0-15$ \\
\cline { 2 - 5 } & \multicolumn{5}{c}{ Level-2 } \\
\cline { 2 - 5 } & 31 & 0.45 & 0.51 & $0-1^{1}$ \\
Depression/Anxiety & 31 & 0.74 & 0.44 & $0-1^{1}$ \\
Medication Use & 28 & 51.93 & 7.77 & $35-60$ \\
Working Alliance & 29 & 1.97 & 0.19 & $1-2 \dagger$ \\
Social Support & \multicolumn{5}{c}{} \\
\hline
\end{tabular}

Note. *indicate nominal data. No intake, primary depression, and no medication use were represented by 0 . †indicates ordinal data.

OQ-45 scores. Graphical and mean score data indicate that participants' OQ-45 scores generally decline over time with subsequent treatment sessions (see Table 7 and Figure 2).

Overall, participants started with a total score of 78.29, which was statistically significantly different from zero, $p<.001$ (Hedge's $g=5.88$; see Table 8). The slope indicates a decrease in OQ-45 score of 1.75 for every one session and this was statistically significantly different from zero, $\mathrm{p}<.001$ (Hedge's $g=2.24$ ). Thus, there was a statistically significant reduction in participants' OQ-45 scores from pre to post treatment (see Figures 3 and 4). 
Table 7

Total OQ-45 Mean Scores by Session

\begin{tabular}{lccc}
\hline Session Number & $M$ & $N$ & $S D$ \\
\hline Intake & 84.65 & 17 & 24.49 \\
Session 1 & 69.85 & 26 & 28.78 \\
Session 2 & 81.13 & 8 & 20.12 \\
Session 3 & 95.50 & 2 & 27.58 \\
Session 4 & 63.35 & 20 & 26.16 \\
Session 5 & 75.33 & 6 & 16.49 \\
Session 6 & 78.00 & 3 & 35.03 \\
Session 7 & $59.15 \dagger$ & 13 & 17.82 \\
Session 8 & $48.29 \dagger$ & 7 & 18.06 \\
Session 9 & 66.29 & 7 & 26.61 \\
Session 10 & $55.75 \dagger$ & 12 & 13.67 \\
Session 11 & $26.00 \dagger$ & 2 & 2.83 \\
Session 13 & $56.63 \dagger$ & 8 & 11.34 \\
Session 14 & $54.13 \dagger$ & 8 & 30.05 \\
Session 15 & $57.00 \dagger$ & 1 & - \\
Session 16 & $59.00 \dagger$ & 1 & - \\
\hline
\end{tabular}

Note. $\uparrow$ Below the OQ-45 clinical cut-off of 63 . 


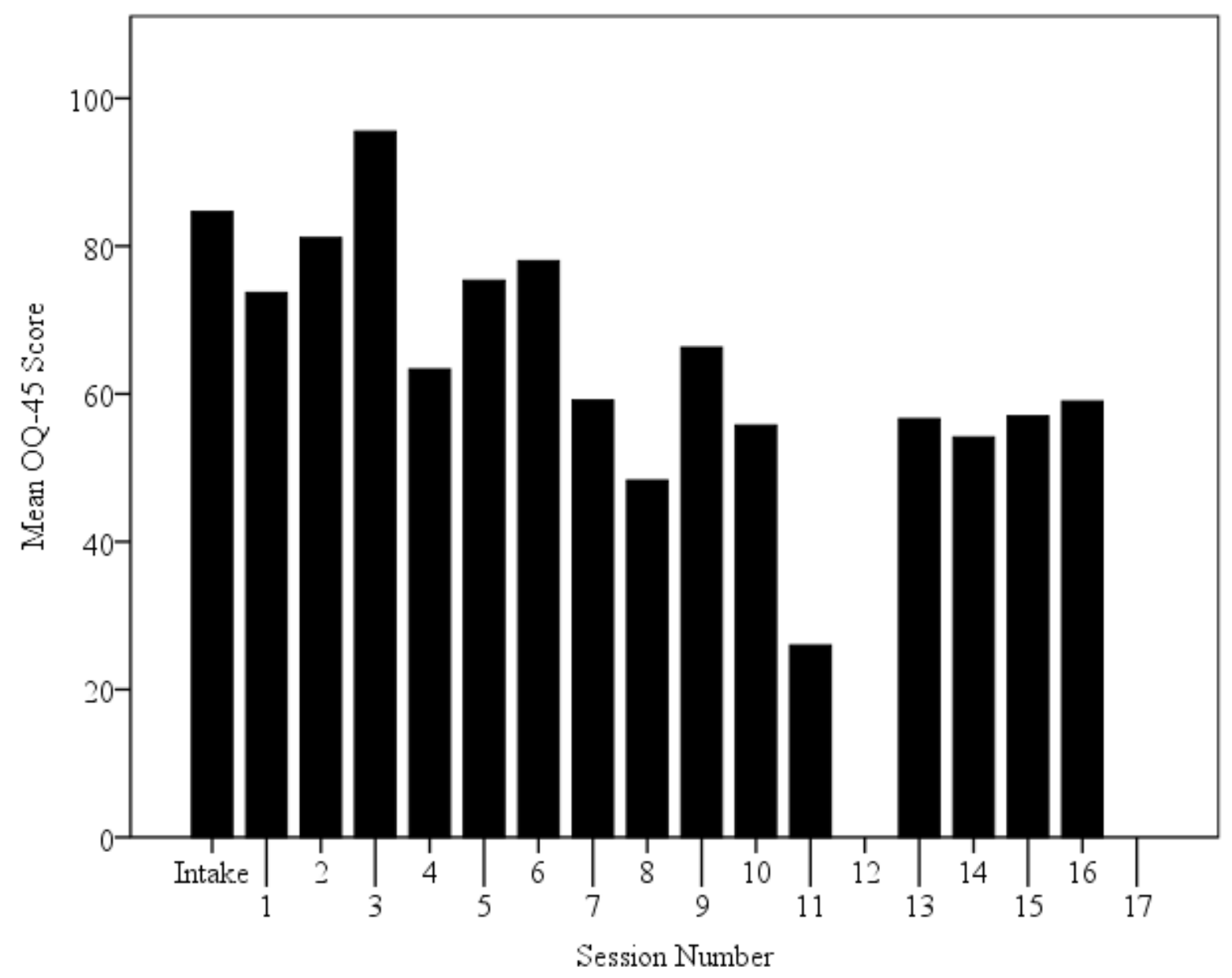

Figure 2. OQ-45 total means by session number. 
Table 8

Results of Hierarchical Linear Modeling for the $O Q-45(N=31)$

\begin{tabular}{|c|c|c|c|c|c|c|c|}
\hline Fixed Effect & $\beta$ & Coefficient & $S E$ & $t$ & $d f$ & $p$-value & Hedge's g \\
\hline \multicolumn{8}{|l|}{ OQ-45 } \\
\hline Intercept & $\beta_{00}$ & 78.29 & 4.78 & 16.38 & 30 & $.001 *$ & 5.88 \\
\hline \multicolumn{8}{|l|}{ Session Number } \\
\hline Slope & $\beta_{10}$ & -1.75 & 0.28 & -6.23 & 30 & $.001 *$ & 2.24 \\
\hline \multicolumn{8}{|c|}{ Waitlist Versus Treatment } \\
\hline Intercept & $\beta_{00}$ & 80.23 & 5.28 & 15.20 & 30 & $.001 *$ & 5.46 \\
\hline Time 1 Slope & $\beta_{10}$ & -4.54 & 3.47 & -1.31 & 30 & .200 & 0.47 \\
\hline Time 2 Slope & $\beta_{20}$ & -1.51 & 0.23 & -6.1 & 30 & $.001 *$ & 2.19 \\
\hline \multicolumn{8}{|l|}{ Depression/Anxiety } \\
\hline \multirow[t]{2}{*}{ Intercept } & $\beta_{00}$ & 85.78 & 6.86 & 12.51 & 29 & $.001 *$ & 4.49 \\
\hline & $\beta_{01}$ & -16.50 & 8.90 & -1.86 & 29 & .074 & 0.67 \\
\hline \multirow[t]{2}{*}{ Slope } & $\beta_{10}$ & -1.92 & 0.43 & -4.43 & 29 & $.001 *$ & 1.59 \\
\hline & $\beta_{11}$ & 0.34 & 0.54 & 0.64 & 29 & .529 & 0.23 \\
\hline \multicolumn{8}{|l|}{ Medication Use } \\
\hline \multirow[t]{2}{*}{ Intercept } & $\beta_{00}$ & 78.18 & 8.93 & 8.77 & 29 & $.001 *$ & 3.15 \\
\hline & $\beta_{01}$ & 0.168 & 10.55 & 0.02 & 29 & .988 & 0.01 \\
\hline \multirow[t]{2}{*}{ Slope } & $\beta_{10}$ & -1.53 & 0.23 & -6.73 & 29 & $.001 *$ & 2.42 \\
\hline & $\beta_{11}$ & -0.29 & 0.43 & -0.68 & 29 & .505 & 0.24 \\
\hline \multicolumn{8}{|c|}{ Working Alliance Inventory } \\
\hline \multirow[t]{2}{*}{ Intercept } & $\beta_{00}$ & 79.02 & 4.76 & 16.60 & 26 & $.001 *$ & 6.27 \\
\hline & $\beta_{01}$ & -0.53 & 0.77 & -0.69 & 26 & .494 & 0.26 \\
\hline \multirow[t]{2}{*}{ Slope } & $\beta_{10}$ & -1.75 & 0.27 & -6.46 & 26 & $.001^{*}$ & 2.44 \\
\hline & $\beta_{11}$ & -0.04 & 0.04 & -1.24 & 26 & .225 & 0.47 \\
\hline \multicolumn{8}{|l|}{ Social Support } \\
\hline \multirow[t]{2}{*}{ Intercept } & $\beta_{00}$ & 56.17 & 56.47 & 1.00 & 27 & .329 & 0.37 \\
\hline & $\beta_{01}$ & 10.93 & 28.61 & 0.38 & 27 & .705 & 0.14 \\
\hline \multirow[t]{2}{*}{ Slope } & $\beta_{10}$ & 0.51 & 2.80 & 0.18 & 27 & .857 & 0.07 \\
\hline & $\beta_{11}$ & -1.17 & 1.43 & -0.82 & 27 & .420 & 0.30 \\
\hline
\end{tabular}

Note. ${ }^{*} p<.001$. Time $1=$ Intake to session 1 . Time $2=$ Session $1-16$. No intake, primary depression, no medication use, average mean WAI, and no social support were the reference groups. All data reported are with robust standard errors except for social support which could not be calculated. 


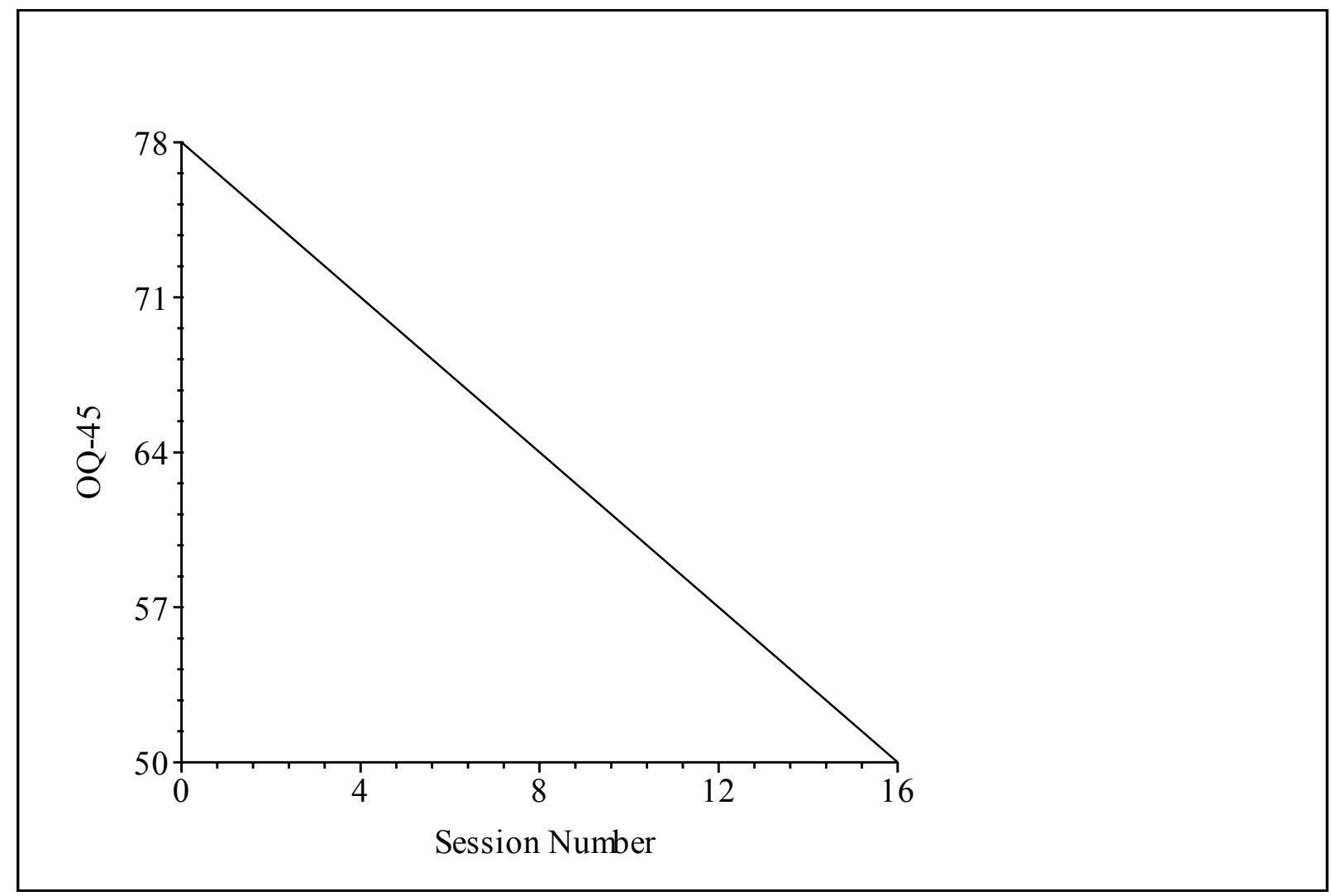

Figure 3. Mean OQ-45 scores by session number. 


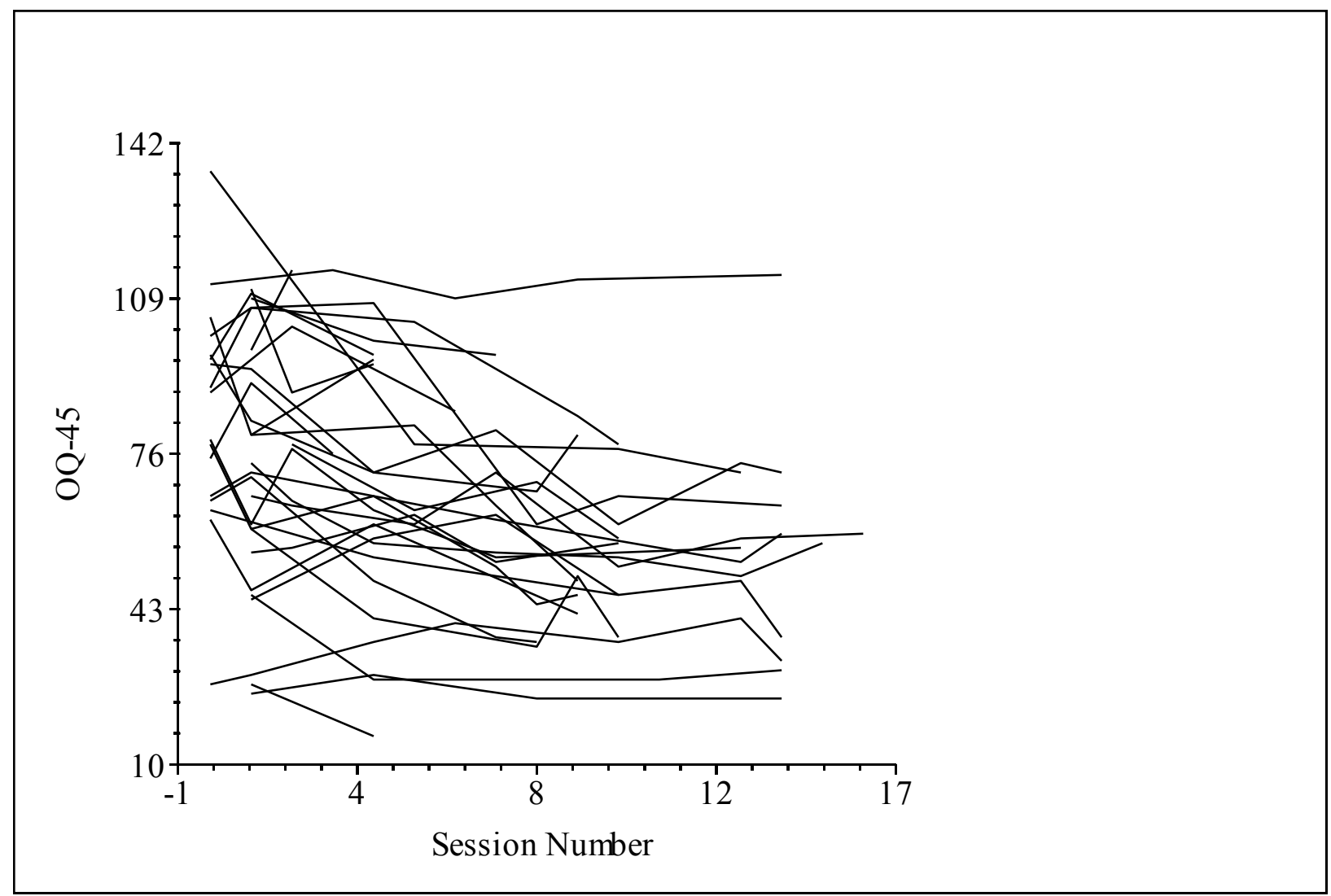

Figure 4. Participants' individual OQ-45 trajectories over time.

Waitlist versus treatment. Results indicate that at intake participants started with a score that was statistically significantly different from 0 score of $80.23, p<.001$. Between intake and session 1, participants experienced a decrease in OQ-45 of 4.54 points but this was not a statistically significant change. Between session 1 and participants' last session, participants experienced a decline on the OQ-45 at a rate of 1.51 points per session, which was statistically significant. In sum, there were no significant differences in participants' scores while they were on the waitlist, though there were significant differences between the start and end of treatment (see Figure 5). 


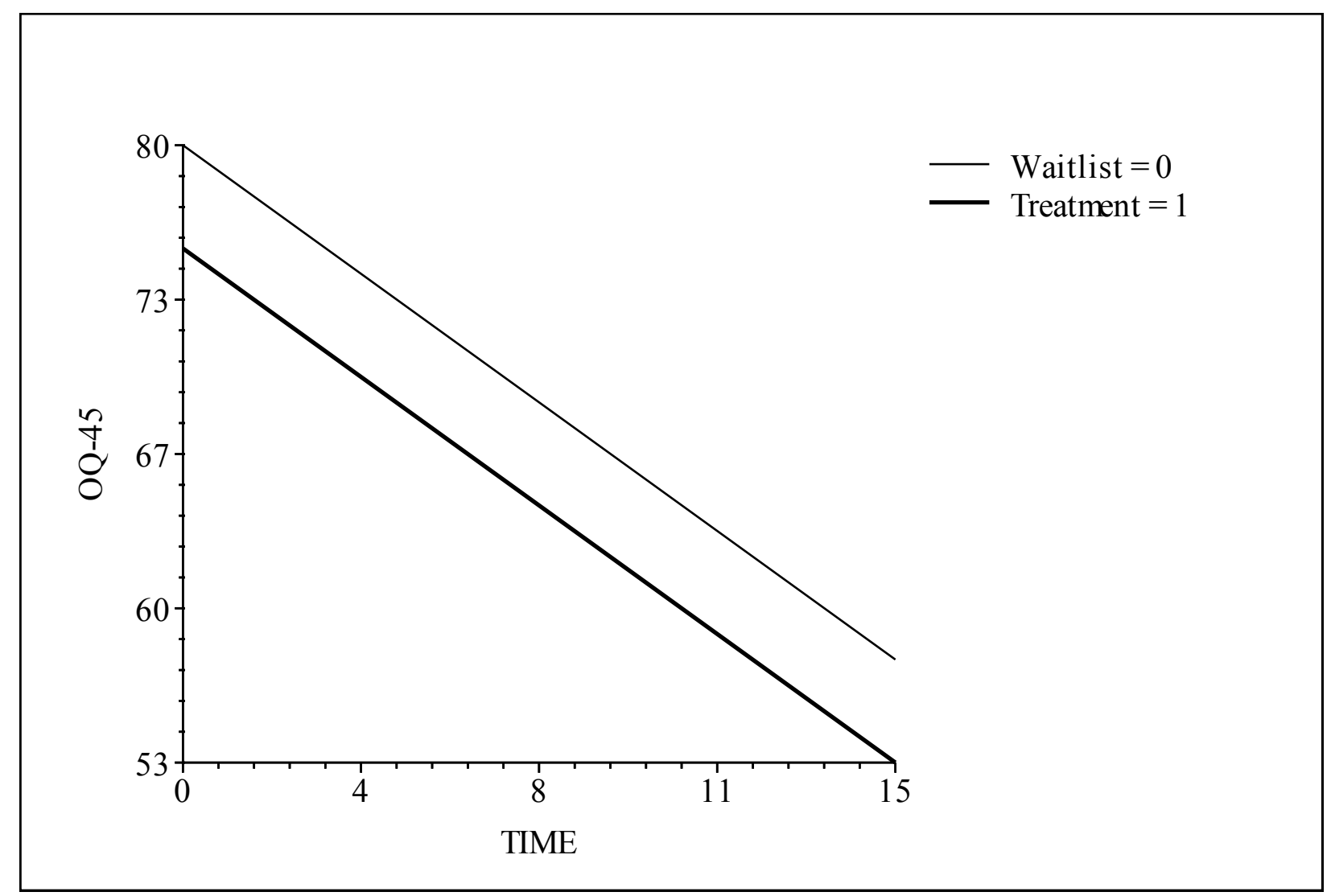

Figure 5. OQ-45 trajectories over time for participants with and without intake data.

Depression versus anxiety. Participants with a primary depressive disorder diagnosis had an average score of 85.78 at intake, which was statistically significantly different from zero, $p<.001$. Individuals with a primary anxiety disorder diagnosis had initial scores of 69.27 that were 16.50 points lower, but this was not statistically significant. Participants with depression experienced a decrease of 1.92 points per session, which was significantly different from zero. Individuals with anxiety experienced a slightly slower rate of 1.58 points per session. However, this was not significantly difference than those with depression. Thus, although this sample was heterogeneously comprised of individuals with a primary depressive and primary anxiety diagnoses, there were no significant differences between the two groups with regards to initial OQ-45 scores or rates of change (see Figure 6). 


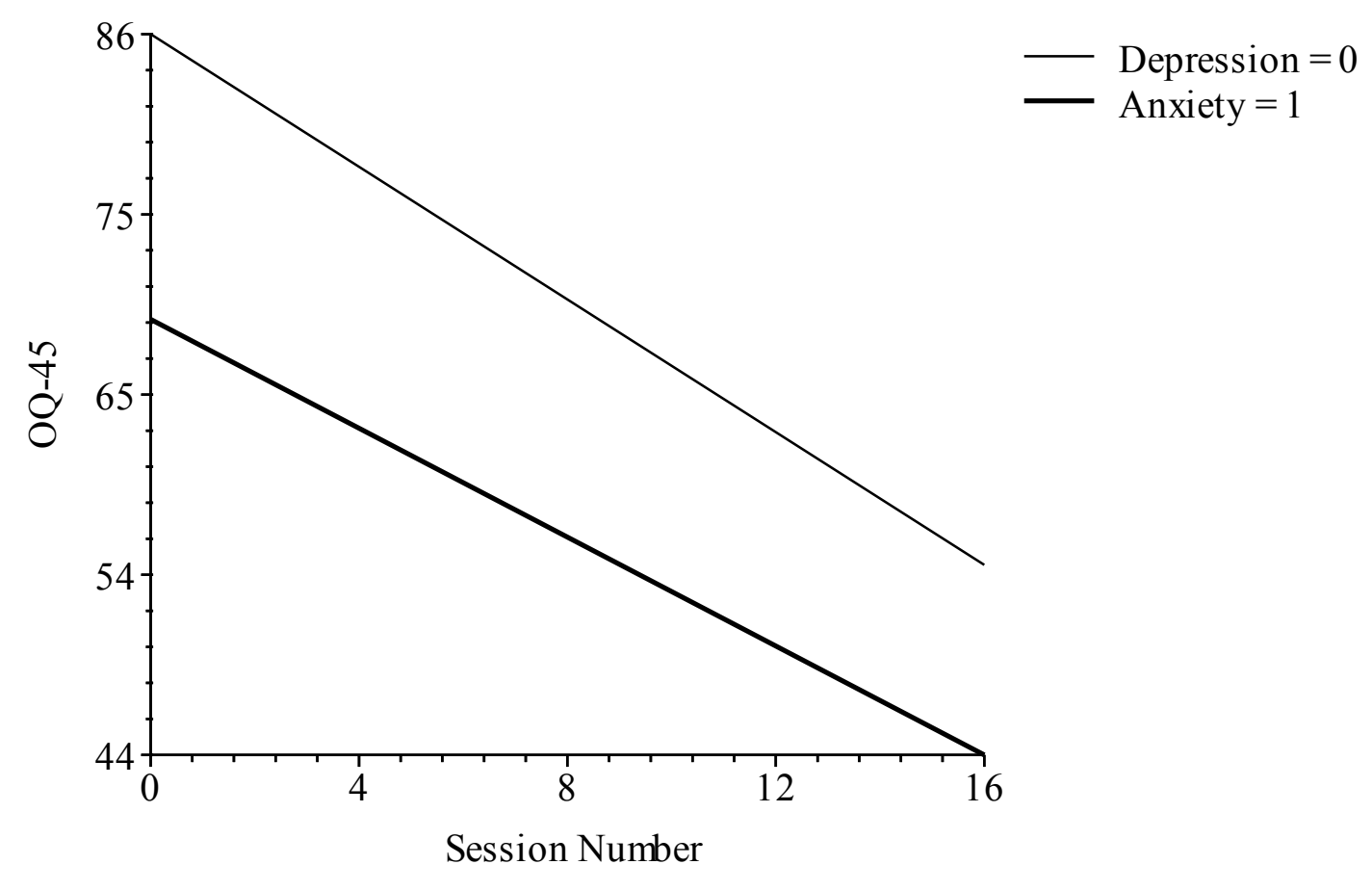

Figure 6. OQ-45 trajectories over time for participants a primary depressive disorder versus primary anxiety disorder.

Medication use. Results indicate participants not taking medication start with an OQ-45 score of 78.18, which was statistically significantly different from zero. Individuals taking medication had higher initial scores of 78.34 ( 0.16 points higher), but this difference was not statistically significant from those not taking medication. Participants not taking medication experienced a decrease in OQ-45 of 1.53 points per one session which was significantly different from zero. Those taking medication experienced a faster decline in scores at 1.82 points per session; however, this difference was not statistically significant. That is, participants taking medication started treatment with higher OQ-45 scores and improved more quickly than those not taking medication, but this difference was not statistically significant (see Figure 7). 


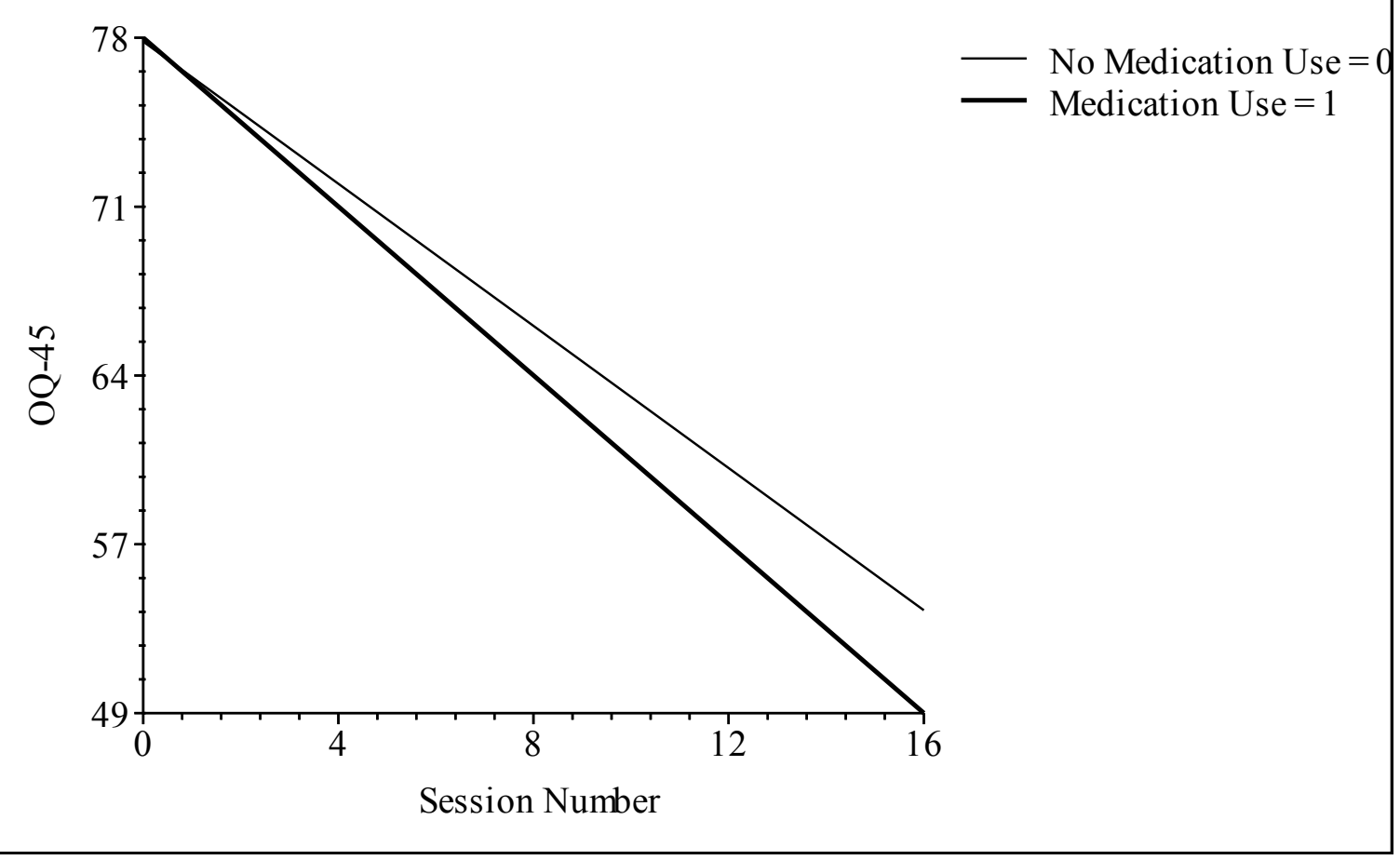

Figure 7. OQ-45 trajectories over time in relation to medication use.

Working alliance with graduate student psychotherapist. The administration of the WAI did not occur every three sessions as intended, and instead it was administered more sporadically and resulted in missing data. Three participants did not complete the WAI at any session. There was no significant difference between WAI scores at pre $(M=51.29 ; S D=9.42)$ and post $(M=54.43 ; S D=7.65), t(13)=-1.89, p=.081$, Hedge's $g=0.37)$. As such, participants' mean WAI scores were used to represent working alliance $(M=52.57 ; S D=7.21)$, which were also not statistically different from pre or post treatment scores. Because WAI scores are virtually never 0 , data were grand mean centred.

Results indicate that participants with average WAI means started with a score of 79.02, which was statistically significantly different from zero. With every increase of 1 point on the WAI, participants' starting OQ-45 scores declined an additional 0.53; however, this difference was not statistically significant. Participants with average WAI mean scores experienced a 
decrease of 1.75 in OQ-45 per session, which was statistically significantly different from zero. With every one point increase on the WAI, participants' OQ-45 scores decreased more quickly at a rate of 1.79 per session, but this was not statistically significant difference. In other words, participants with positive working alliance improve faster than those with poorer working alliance, though this was not a statistically significant difference (see Figure 8).

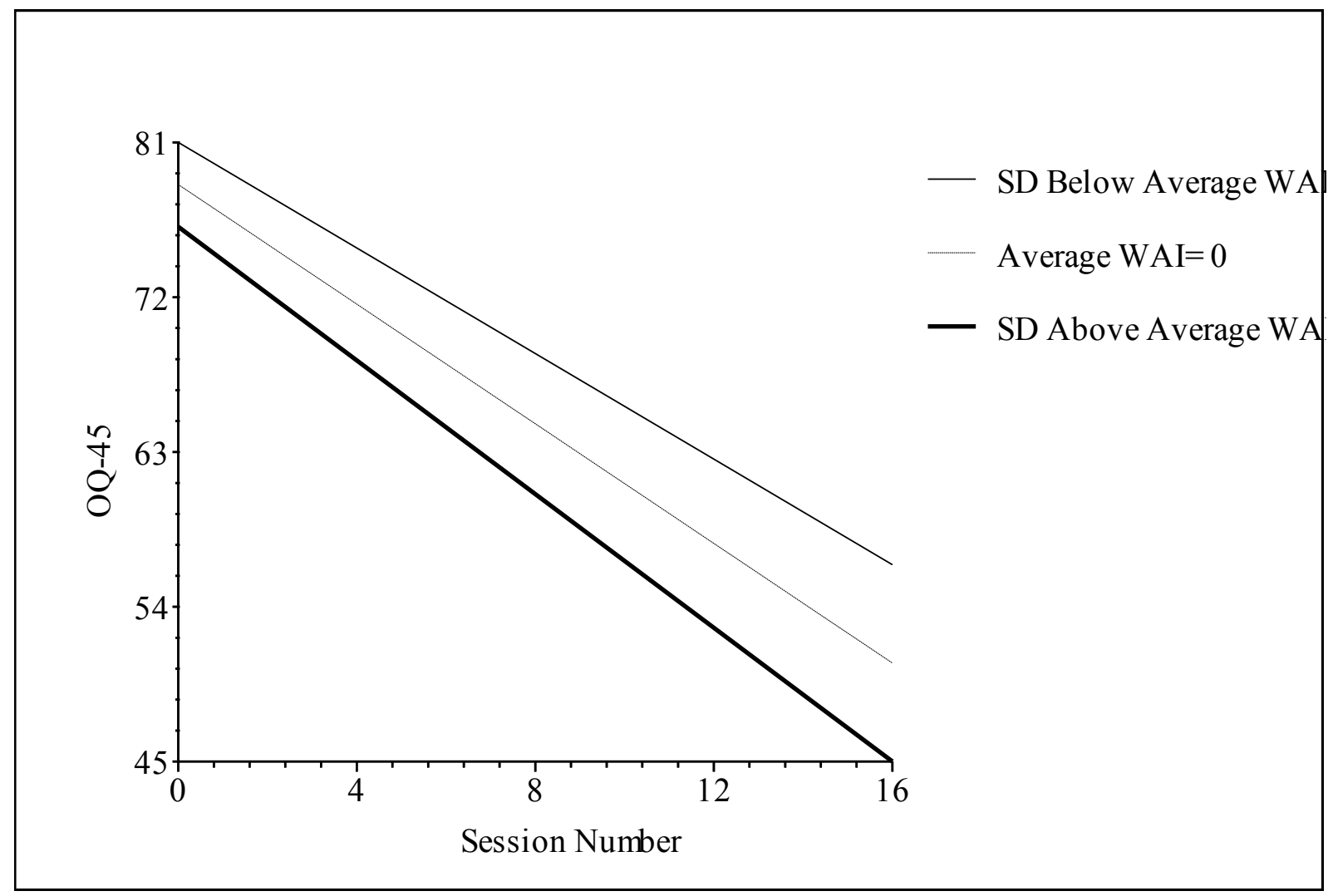

Figure 8. OQ-45 trajectories over time in relation to working alliance with graduate student psychotherapist. $\mathrm{SD}=$ standard deviation.

Social support. Robust standard error data could not be computed for social support, similar to the binary logistic regression. Thus, the less stringent standard error is presented and should be interpreted with caution. As well, data for social support were not available for two individuals. Participants with no social support had an average OQ-45 at start of 56.17, though this was not statistically different from zero. With increased social support, participants 
experienced an increase OQ-45 score of 10.93, but this is not statistically significant. OQ-45 scores increased by 0.51 per session for those without social support, but this was not statistically significantly different from zero. With increased social support, participants experienced a faster decline of 0.66 points per session. However, this was also not statistically significant. This suggests that participants' symptoms of mental health may improve with increased social support. However, it is difficult to determine the actual impact of social support because virtually all participants had some form of it in their lives (see Figure 9). Further research may benefit from exploring more nuanced differences in social support rather than its presence or absence.

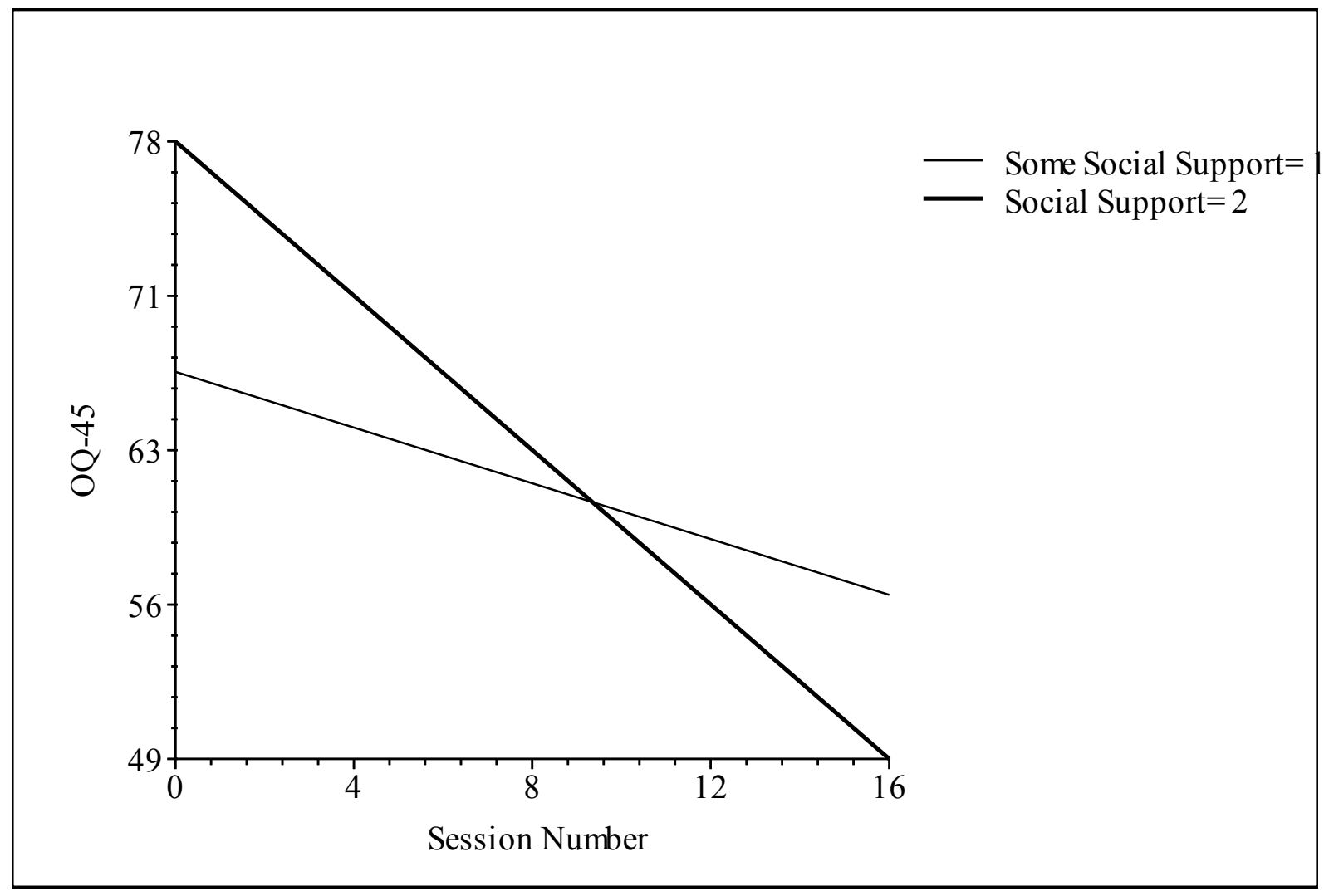

Figure 9. OQ-45 trajectories over time in relation to social support.

DASS-21 clinical cut-offs. From pre to post treatment, participants' overall scores ranged from decreasing by 66 to increasing by $36(M=-11.77 ; S D=24.23)$. Cut-off scores for 
the DASS-21 are particular to each of the three factors, Depression, Anxiety, and Stress, and therefore are examined separately. Although each factor has symptom severity cut-offs indicating either normal, mild, moderate, severe, or extremely severe ranges, the cut-off for each is distinct.

From pre to post treatment, participants experienced changes ranging from a decrease of 24 points to an increase of eight points $(M=-5.37 ; S D=7.32)$ for Depression (see Table 9). A Wilcoxon Signed Ranked Test indicates that changes in severity from pre $(M d n=3.00 ; S D=$ 1.63) to post $(M d n=1.50 ; S D=1.71)$ were statistically significant, $z=3.00, p=.004$. Thus, participants' symptom severity for depression reduced significantly as a result of treatment. For Anxiety, participants' scores changes from pre to post ranged from reducing by 28 points to increasing by 20 points $(M=-3.13 ; S D=9.20)$. With regards to symptom severity, participants did not experience a statistically significant decline from pre $(M d n=2.50 ; S D=1.69)$ to post $(M d n=1.50 ; S D=1.61), z=-1.24, p=.214$. Lastly, with regards to Stress from pre to post treatment, participants experienced a range from a decline of 22 to an increase of 20. Symptom severity from pre $(M d n=2.00 ; S D=1.33)$ to post $(M d n=1.50 ; S D=1.22)$ was not statistically significant, $z=-1.32, p=.187$. In sum, changes in symptom severity from pre to post treatment were significant for Depression and but not for Anxiety or Stress. 
Table 9

Frequency of Pre-Post Treatment DASS-21 Scores in Relation to Clinical Cut-Offs $(N=30)$

\begin{tabular}{lcccccccc}
\hline Factor & Normal & Mild & Moderate & Severe & $\begin{array}{c}\text { Extremely } \\
\text { Severe }\end{array}$ & $z$ & $p$ & $r$ \\
\hline $\begin{array}{l}\text { Depression } \\
\text { Pre }\end{array}$ & 8 & 4 & 7 & 1 & 10 & 3.00 & $.004^{*}$ & 0.39 \\
$\quad$ Post & 15 & 4 & 3 & 0 & 8 & & & \\
$\begin{array}{c}\text { Anxiety } \\
\text { Pre }\end{array}$ & 12 & 3 & 5 & 2 & 8 & -1.24 & .214 & 0.16 \\
Post & 15 & 2 & 4 & 4 & 5 & & & \\
Stress & 10 & 6 & 8 & 3 & 3 & -1.32 & .187 & 0.17 \\
Pre & 15 & 5 & 4 & 6 & 0 & & & \\
Post & 15 & & & & & & & \\
\hline
\end{tabular}

Note. ${ }^{*}$ indicates $p<.01$

DASS-21 HLM. It was hypothesized that participants would experience a significant reduction in symptoms of depression and anxiety as measured by the DASS-21 over the course of individual CBT. DASS-21 scores (level-1 outcome variable) were explored, with medication use, social support, and working alliance used as level-2 predictor variables. Similar to the analyses with the OQ-45, differences in participants' scores while on the waitlist versus in treatment were examined using a piecewise HLM analysis with Time 1 indicating intake versus session 1 and Time 2 indicating session 1 to last session. Time components (i.e. session number) and parameters (e.g. session numbers uncentred, average WAI grand mean centred) were the same as for the OQ-45. For a summary of DASS-21 descriptive HLM statistics, see Table 10 . 
Table 10

Descriptive Statistics for HLM for the DASS-21, Session Number, and Predictor Variables

\begin{tabular}{lcccc}
\hline Variable & $N$ & $M$ & $S D$ & Range \\
\hline \multirow{3}{*}{ Sessions } & \multicolumn{4}{c}{ Level-1 } \\
\cline { 2 - 5 } DASS-21 Total & 152 & 5.00 & 4.61 & $0-16$ \\
Intake Versus Session 1 & 139 & 41.58 & 26.48 & $1-118$ \\
Session 1 versus Last Session & 150 & 0.80 & 0.40 & $0-1$ \\
\cline { 2 - 5 } & 150 & 4.17 & 4.44 & $0-15$ \\
\cline { 2 - 5 } Depression/Anxiety & 31 & 0.45 & 0.51 & $0-1^{1}$ \\
Medication Use & 31 & 0.74 & 0.44 & $0-1^{1}$ \\
Working Alliance & 28 & 52.61 & 7.23 & $35-60$ \\
Social Support & 29 & 1.90 & 0.41 & $0-2 \uparrow$ \\
\hline
\end{tabular}

Note. ${ }^{1}$ indicate nominal data. No intake, primary depression, and no medication use were represented by 0 . $\dagger$ indicates level of social support where $0=$ No, $1=$ Somewhat, and $2=$ Yes.

DASS-21 scores. Participants' mean DASS-21 appear to reduce over time (see Table 11

and Figure 10). Overall, participants started with a total DASS-21 score of 53.64, which was statistically significantly different from zero, $p<.001$ (Hedge's $g=3.78$; see Table 12). The slope indicates a decrease in DASS-21 scores of 1.81 for every session which was statistically significantly different from zero, $\mathrm{p}<.001$ (Hedge's $g=1.79$ ). Thus, there was a statistically significant reduction in participants' DASS-21 scores from pre to post treatment (see Figures 11 and 12). 
Table 11.

Total DASS-21 Mean Scores by Session

\begin{tabular}{lccc}
\hline Session Number & $M$ & $N$ & $S D$ \\
\hline Intake & 52.00 & 16 & 29.52 \\
Session 1 & 51.73 & 26 & 27.58 \\
Session 2 & 57.11 & 9 & 31.29 \\
Session 3 & 48.33 & 3 & 16.74 \\
Session 4 & 41.16 & 19 & 26.40 \\
Session 5 & 55.67 & 6 & 25.09 \\
Session 6 & 48.00 & 3 & 39.85 \\
Session 7 & 32.67 & 12 & 18.06 \\
Session 8 & 24.00 & 7 & 15.14 \\
Session 9 & 38.67 & 6 & 22.97 \\
Session 10 & 31.33 & 12 & 21.38 \\
Session 11 & 14.00 & 2 & 8.49 \\
Session 13 & 31.50 & 8 & 23.95 \\
Session 14 & 21.38 & 8 & 18.45 \\
Session 15 & 26.00 & 1 & - \\
Session 16 & 38.00 & 1 & - \\
\hline
\end{tabular}




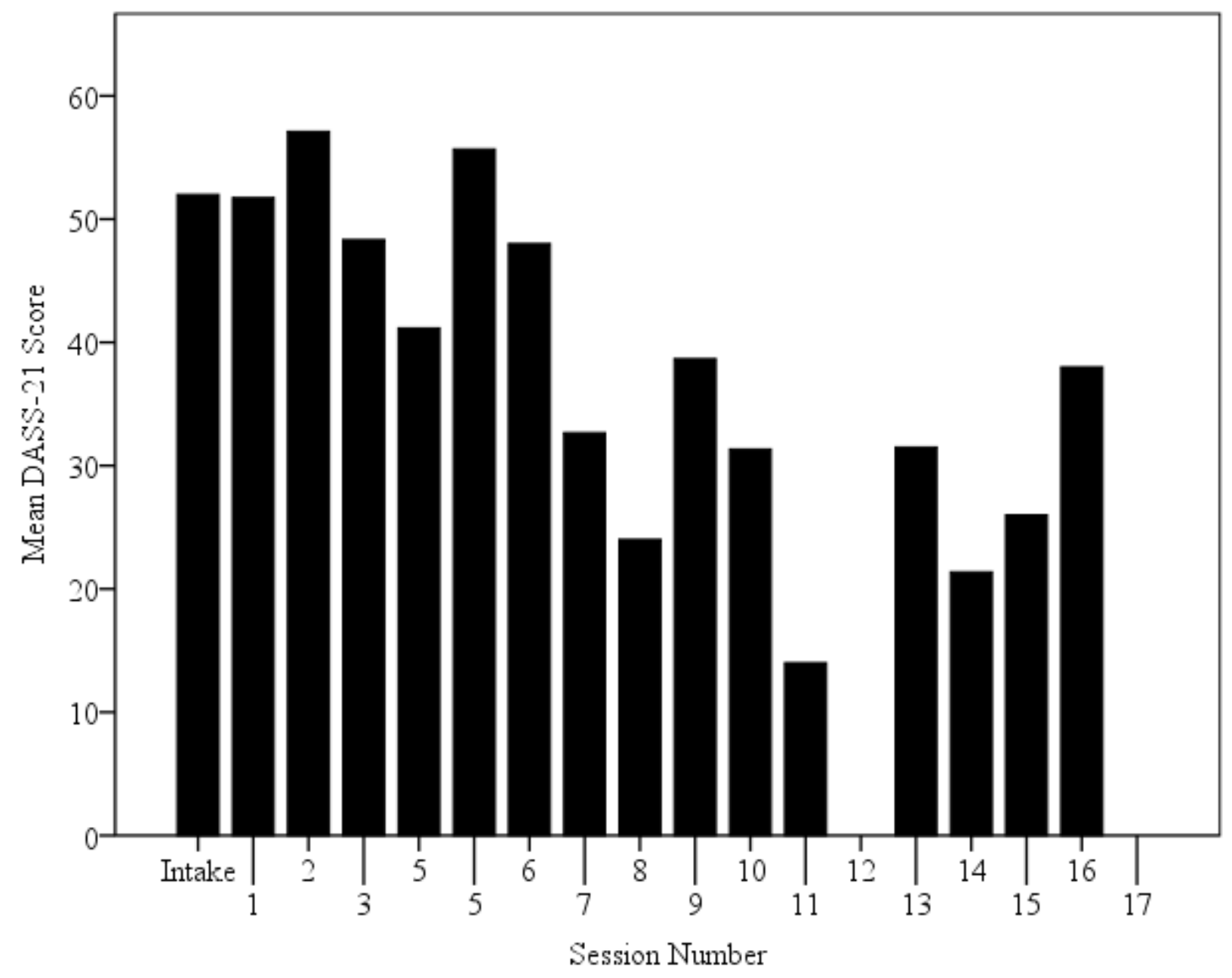

Figure 10. DASS-21 total means by session number. 
Table 12

Results of Hierarchical Linear Modeling for the DASS-21 $(N=31)$

\begin{tabular}{|c|c|c|c|c|c|c|c|}
\hline Fixed Effect & $\beta$ & Coefficient & $S E$ & $t$ & $d f$ & $p$-value & Hedge's $g$ \\
\hline \multicolumn{8}{|l|}{ DASS-21 } \\
\hline Intercept & $\beta_{00}$ & 53.64 & 51.0 & 10.51 & 30 & $.001 * *$ & 3.78 \\
\hline \multicolumn{8}{|l|}{ Session Number } \\
\hline Slope & $\beta_{10}$ & -1.81 & 0.36 & -4.97 & 30 & $.001 * *$ & 1.79 \\
\hline \multicolumn{8}{|c|}{ Waitlist Versus Treatment } \\
\hline Intercept & $\beta_{00}$ & 50.12 & 6.14 & 8.17 & 30 & $.001 * *$ & 2.93 \\
\hline Time 1 Slope & $\beta_{10}$ & 4.18 & 3.73 & 1.21 & 30 & .271 & 0.43 \\
\hline Time 2 Slope & $\beta_{20}$ & -2.02 & 0.34 & -5.92 & 30 & $.001 * *$ & 2.13 \\
\hline \multicolumn{8}{|l|}{ Depression/Anxiety } \\
\hline \multirow[t]{2}{*}{ Intercept } & $\beta_{00}$ & 59.456 & 8.08 & 7.35 & 29 & $.001 * *$ & 2.73 \\
\hline & $\beta_{01}$ & -12.90 & 9.48 & -1.36 & 29 & .184 & 0.49 \\
\hline \multirow[t]{2}{*}{ Slope } & $\beta_{10}$ & -2.06 & 0.60 & -3.41 & 29 & $.002 *$ & 1.22 \\
\hline & $\beta_{11}$ & 0.56 & 0.67 & 0.83 & 29 & .411 & 0.30 \\
\hline
\end{tabular}

Medication Use

Intercept

$\begin{array}{lcccccc}\beta_{00} & 51.78 & 7.71 & 6.72 & 29 & .001 * * & 2.41 \\ \beta_{01} & 2.43 & 9.96 & 0.25 & 29 & .809 & 0.09 \\ \beta_{10} & -1.12 & 0.39 & -2.89 & 29 & .007 * & 1.04 \\ \beta_{11} & -0.87 & 0.60 & -1.45 & 29 & .157 & 0.52\end{array}$

Working Alliance Inventory (WAI)

$\begin{array}{lccccccc}\text { Intercept } & \beta_{00} & 54.04 & 5.27 & 10.25 & 26 & .001 * * & 3.87 \\ & \beta_{01} & -0.46 & 0.74 & -0.62 & 26 & .543 & 0.23 \\ \text { Slope } & \beta_{10} & -1.76 & 0.38 & -4.69 & 26 & .001 * * & 1.77 \\ & \beta_{11} & -0.02 & 0.08 & -0.31 & 26 & .763 & 0.18\end{array}$

Social Support

$\begin{array}{lccccccc}\text { Intercept } & \beta_{00} & 38.68 & 27.17 & 1.42 & 27 & .166 & 0.53 \\ & \beta_{01} & 8.04 & 14.02 & 0.57 & 27 & .571 & 0.21 \\ \text { Slope } & \beta_{10} & 2.50 & 2.59 & 0.96 & 27 & .344 & 0.36 \\ & \beta_{11} & -2.25 & 1.33 & -1.69 & 27 & .103 & 0.63\end{array}$

Note. ${ }^{*} p<.001$. Time $1=$ Intake to session 1 . Time $2=$ Session $1-16$. No intake, primary depression, no medication use, average mean WAI, and no social support were the reference groups. All data reported are with robust standard errors except for social support which could not be calculated. 


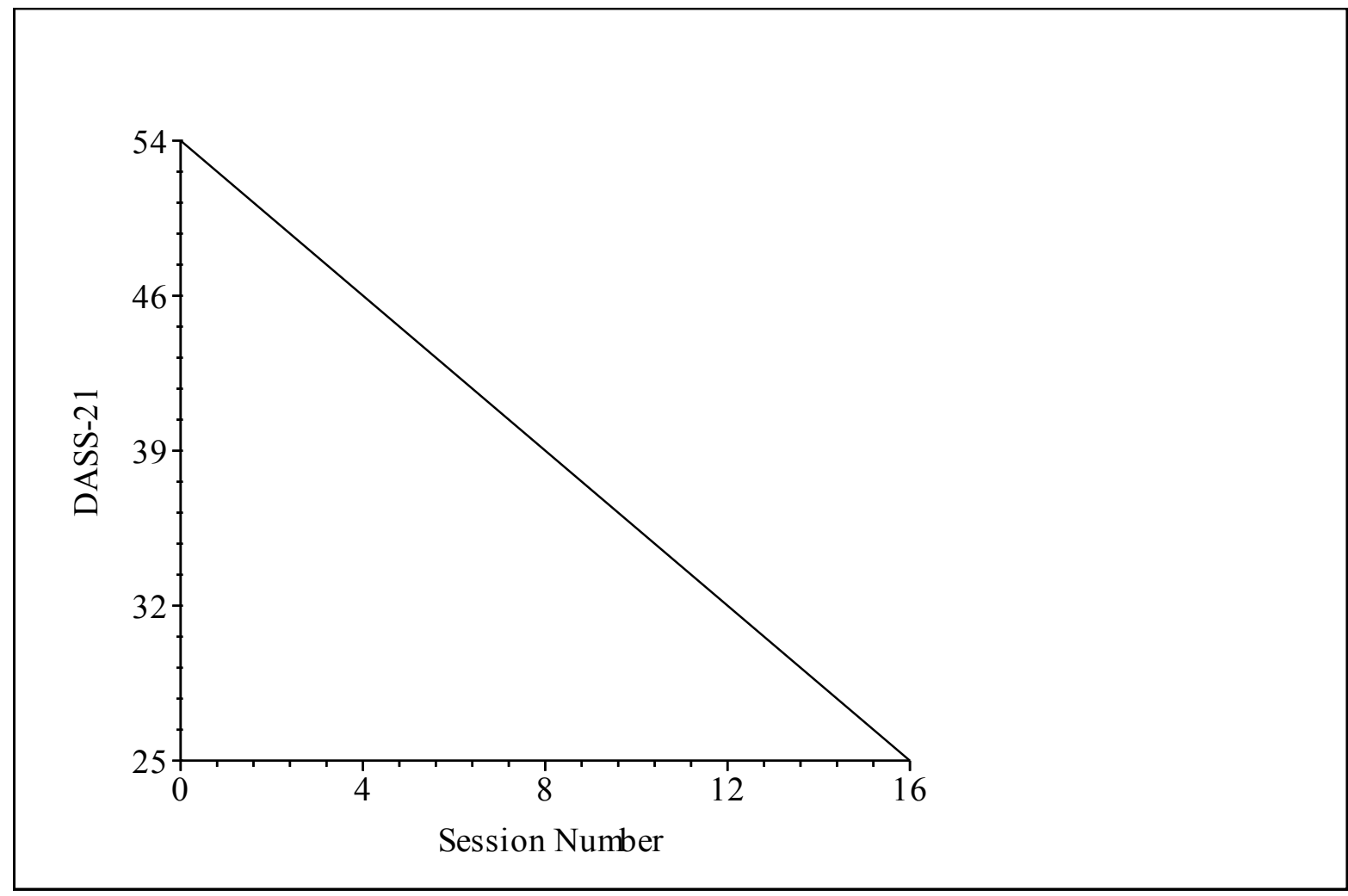

Figure 11. Mean DASS-21 scores by session number.

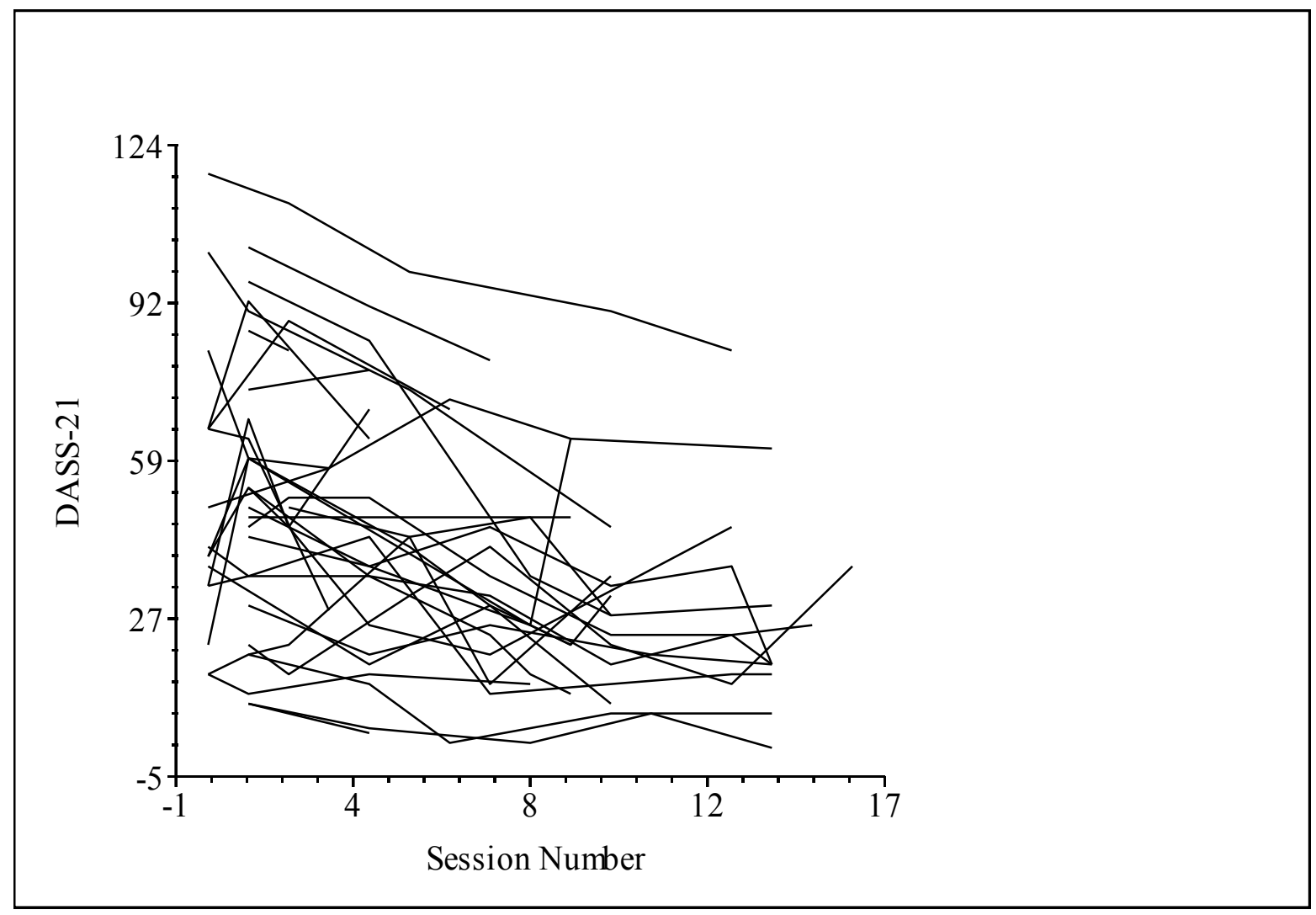

Figure 12. Participants' individual DASS-21 trajectories over time. 
Waitlist versus treatment. Differences in scores while participants were on the waitlist versus in treatment were examined. Participants without intake started with a score of 50.12, which was statistically significantly different from zero. Between intake and session 1 , participants experienced a decrease in DASS-21 of 4.18 points but this was not a statistically significant change. Between session 1 and participants' last session, participants experienced a decline on the OQ-45 at a rate of 2.02 points per session, which was statistically significant. Thus, there were no significant differences in participants' scores while they were on the waitlist, though there were significant differences between pre and post treatment (see Figure 13).

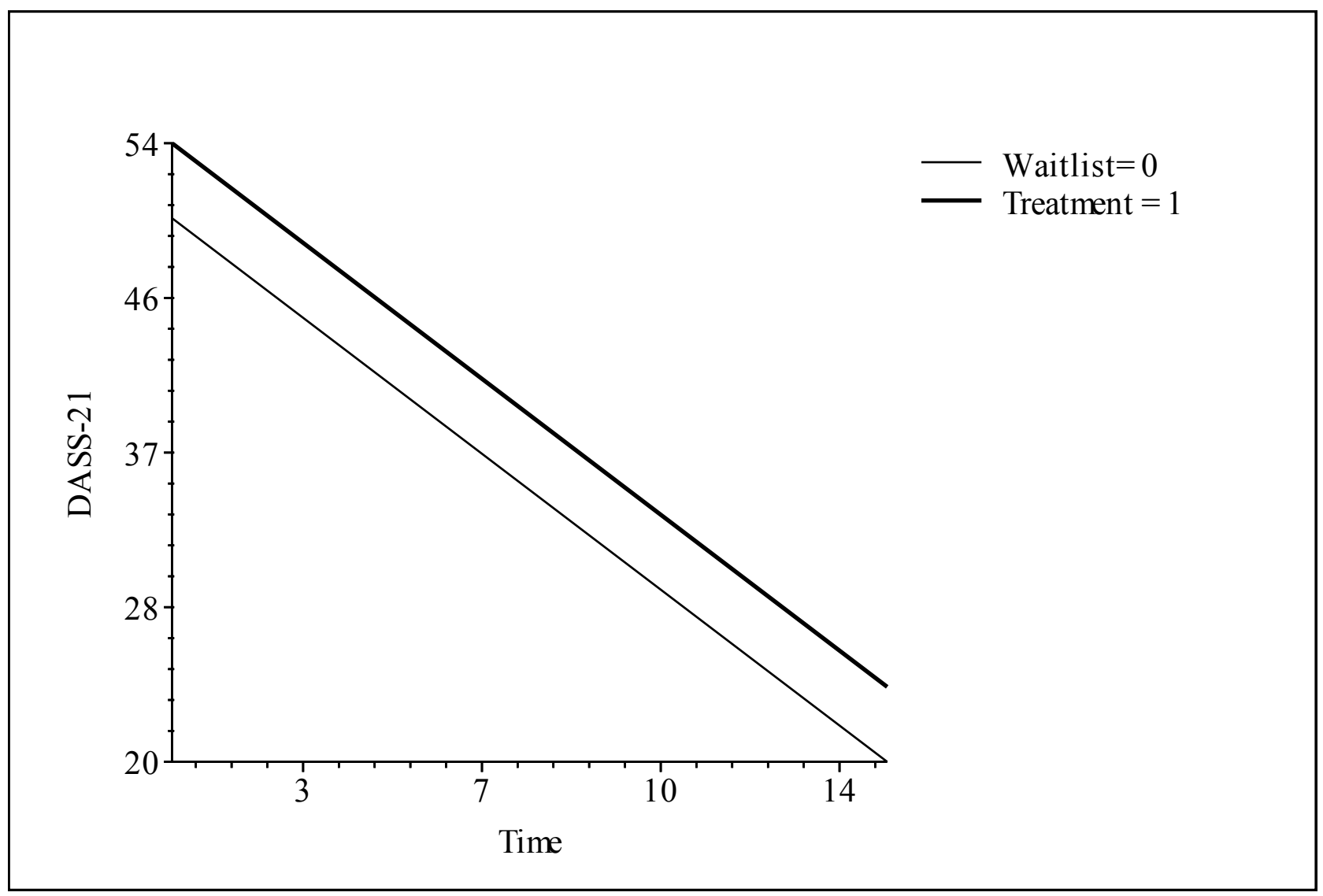

Figure 13. DASS-21 trajectories over time for participants with and without intake data. 
Depression versus anxiety. Individuals with primary depression had an average score of 59.46 at the start, which was statistically significantly different from zero. Those with primary anxiety had initial scores of 46.56 that were 12.90 points lower; however, this difference was not statistically significant. Participants with primary depression experienced a decline on the DASS-21 of 2.06 points per one session, which is statistically significantly different from zero. Similar to the OQ-45, participants with anxiety experienced a slightly slower rate of change by 0.56 points (1.49 per session), but this was not a statistically significantly difference. In sum, there were no significant differences in DASS-21 scores or rate of change between participants with a primary depressive disorder versus primary anxiety disorder (see Figure 14).

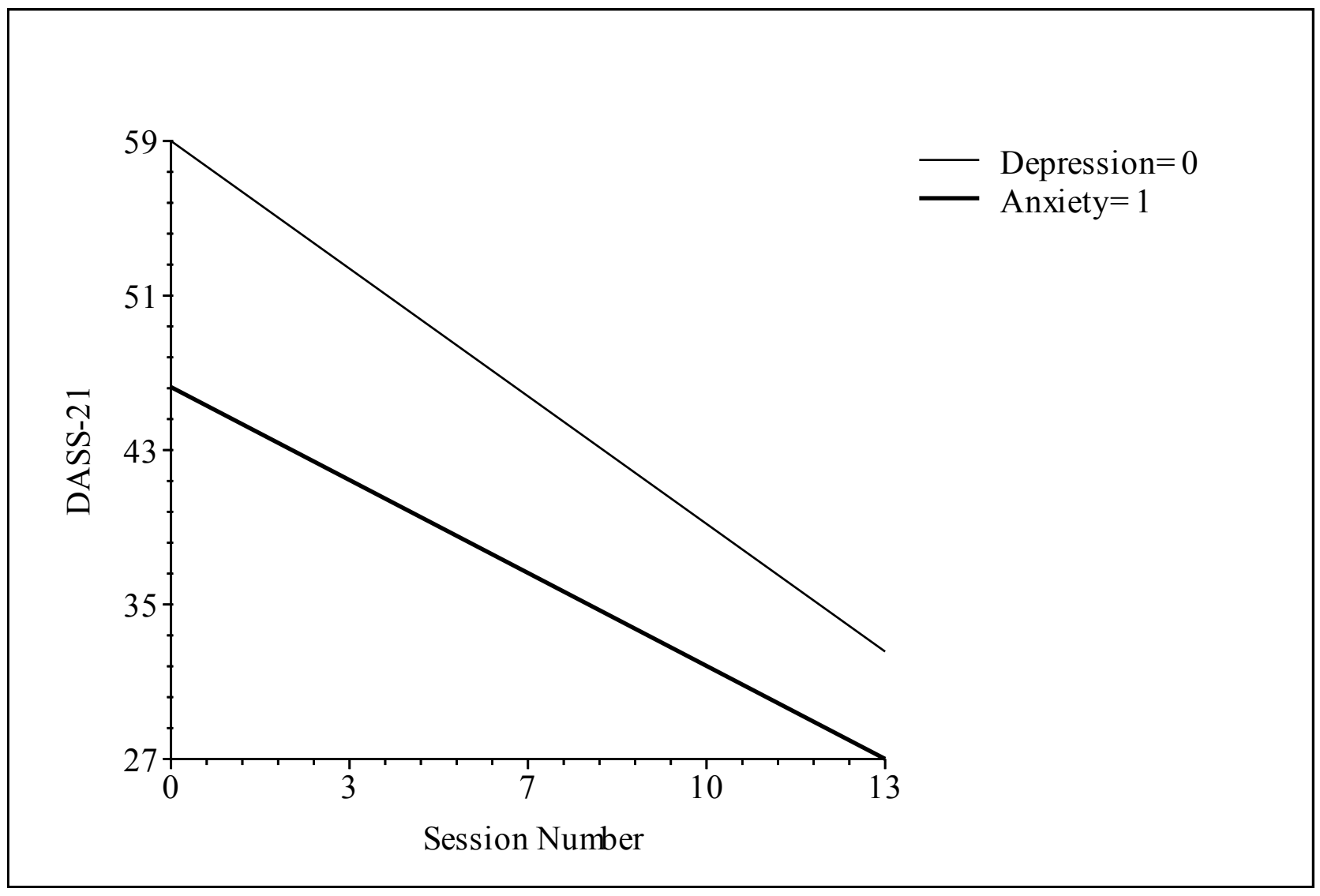

Figure 14. DASS-21 trajectories over time for participants a primary depressive disorder versus a primary anxiety disorder. 
Medication use. Participants who were not taking medication while undergoing CBT started with a DASS-21 score of 51.78, which is statistically significantly different from zero. Participants who were taking medication had higher DASS-21 scores at the start at 54.22, but this difference was not statistically significant. Those not taking medication experienced a decrease on the DASS-21 of 1.12 points per one session, which was statistically significantly difference from zero. Rates of change were faster for those taking medication ( 2.00 points per session), though this difference was not statistically significant. Therefore, medication use does not appear to be a significant predictor of DASS-21 score changes (see Figure 15).

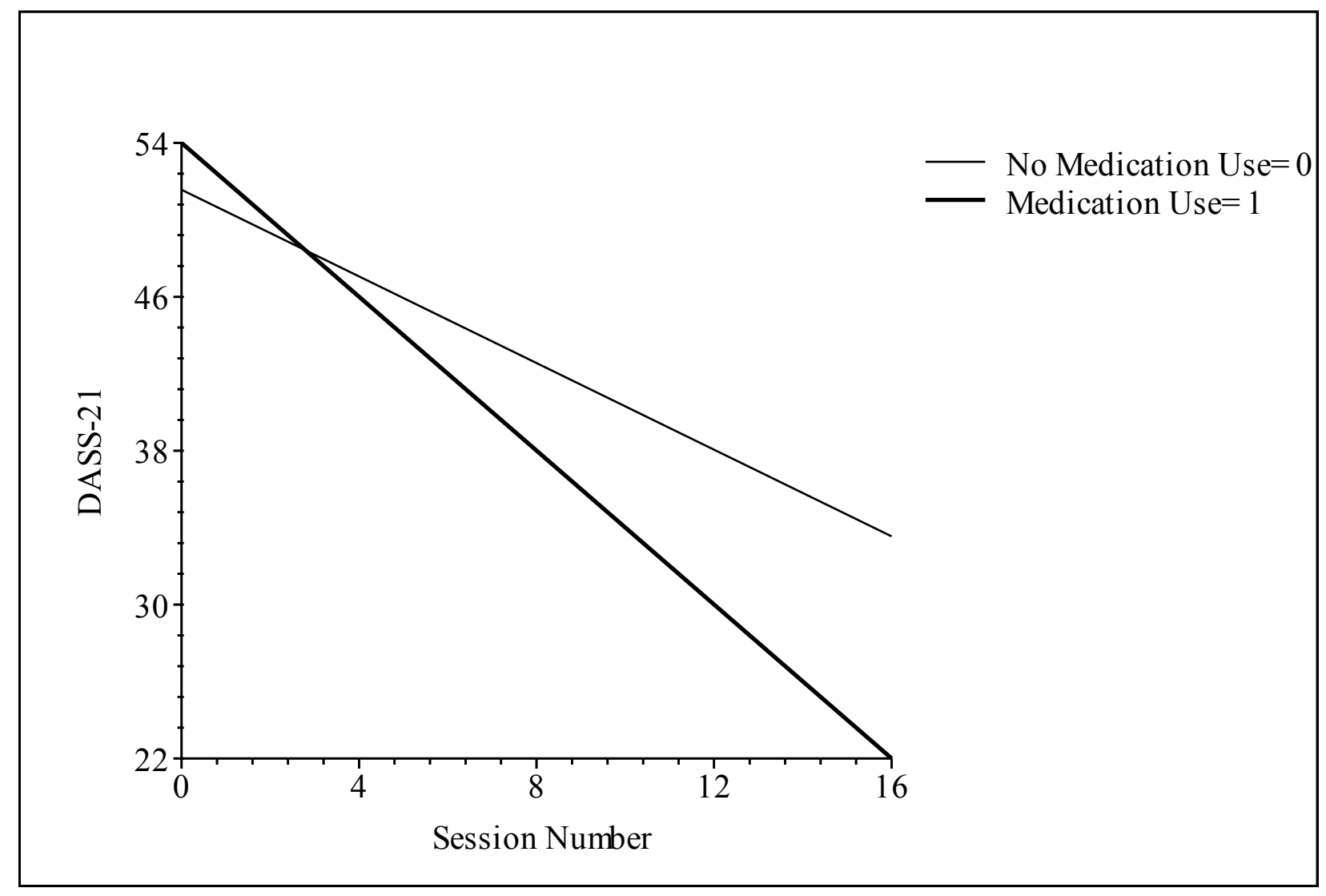

Figure 15. DASS-21 trajectories over time in relation to medication use.

Working alliance with graduate student psychotherapist. As noted above, mean WAI scores were used to represent participants' working alliance with their graduate student psychotherapists. WAI data were missing for three participants. Participants with an average 
WAI means started with a score of 54.04, which was statistically significantly different from zero. As the WAI increased by 1 point, participants' starting DASS- 21 decreased by an additional 0.46 points, although this is not statistically significant. Individuals with average WAI mean scores experienced a decrease of 1.76 per session, which was statistically significantly different from zero. With an increase of 1 point on the WAI, DASS-21 scores decreased by an additional 0.02 points, though this difference was not statistically significant (see Figure 16). Thus, working alliance did not significantly predict change on the DASS-21.

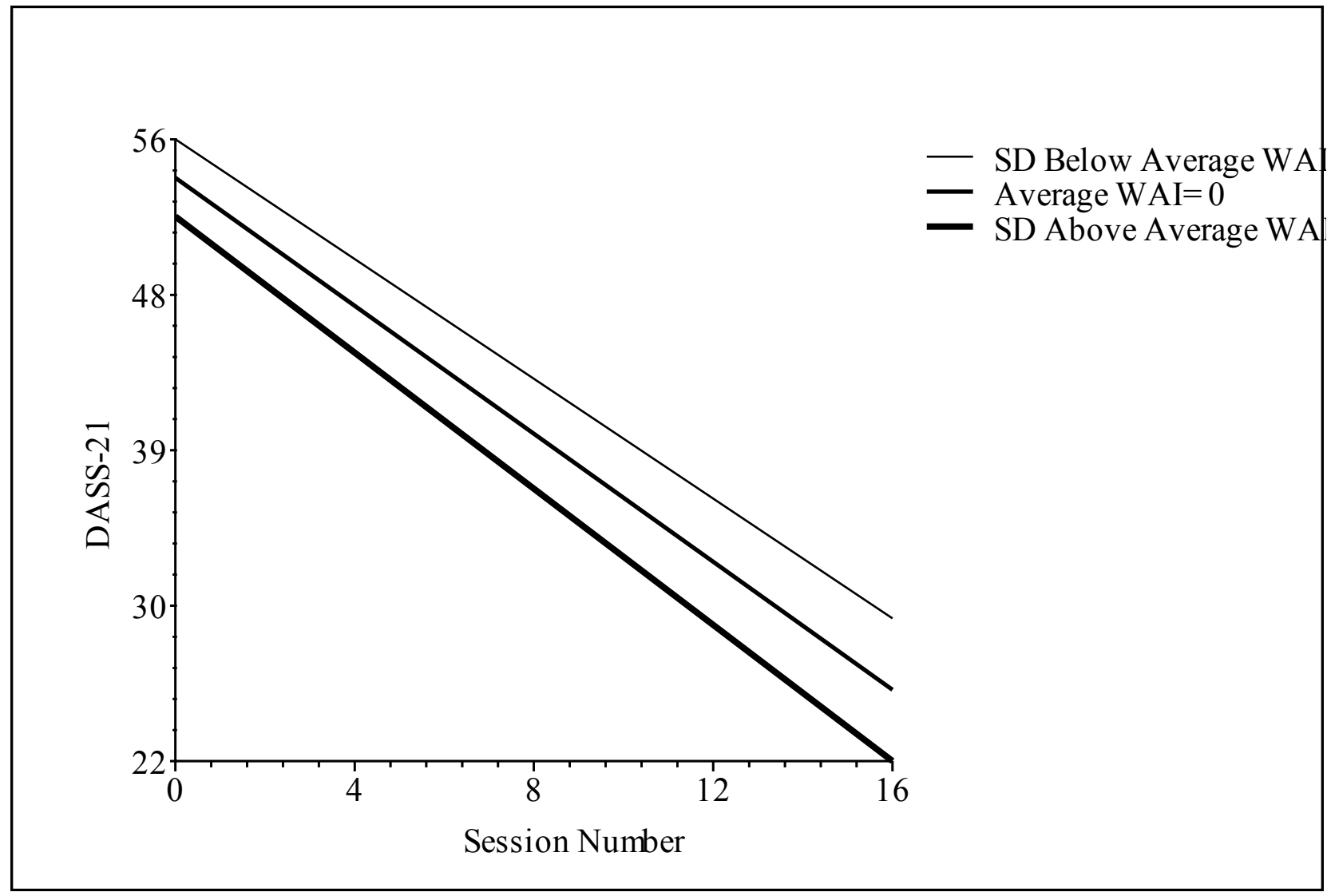

Figure 16. DASS-21 trajectories over time in relation to working alliance with graduate student psychotherapist.

Social support. As with the OQ-45, robust standard error data could not be computed for social support. Final estimation of fixed effects is reported in its place and therefore should be interpreted with caution. It should be noted that social support data were missing for two 
participants. Individuals with no social support had an average DASS-21 at start of 38.68, but this was not statistically significantly different from zero. As social support increased, participants experienced an increase of 8.04 , but this difference was not statistically significant. Participants who reported having no social support had DASS-21 scores increase at a rate of 2.50 per session, though this was not statistically significantly different from zero. Participants with reported greater social support experienced a decline of 0.25 points per session. Again, this was not statistically significant, $p=.103$ (Figure 17).

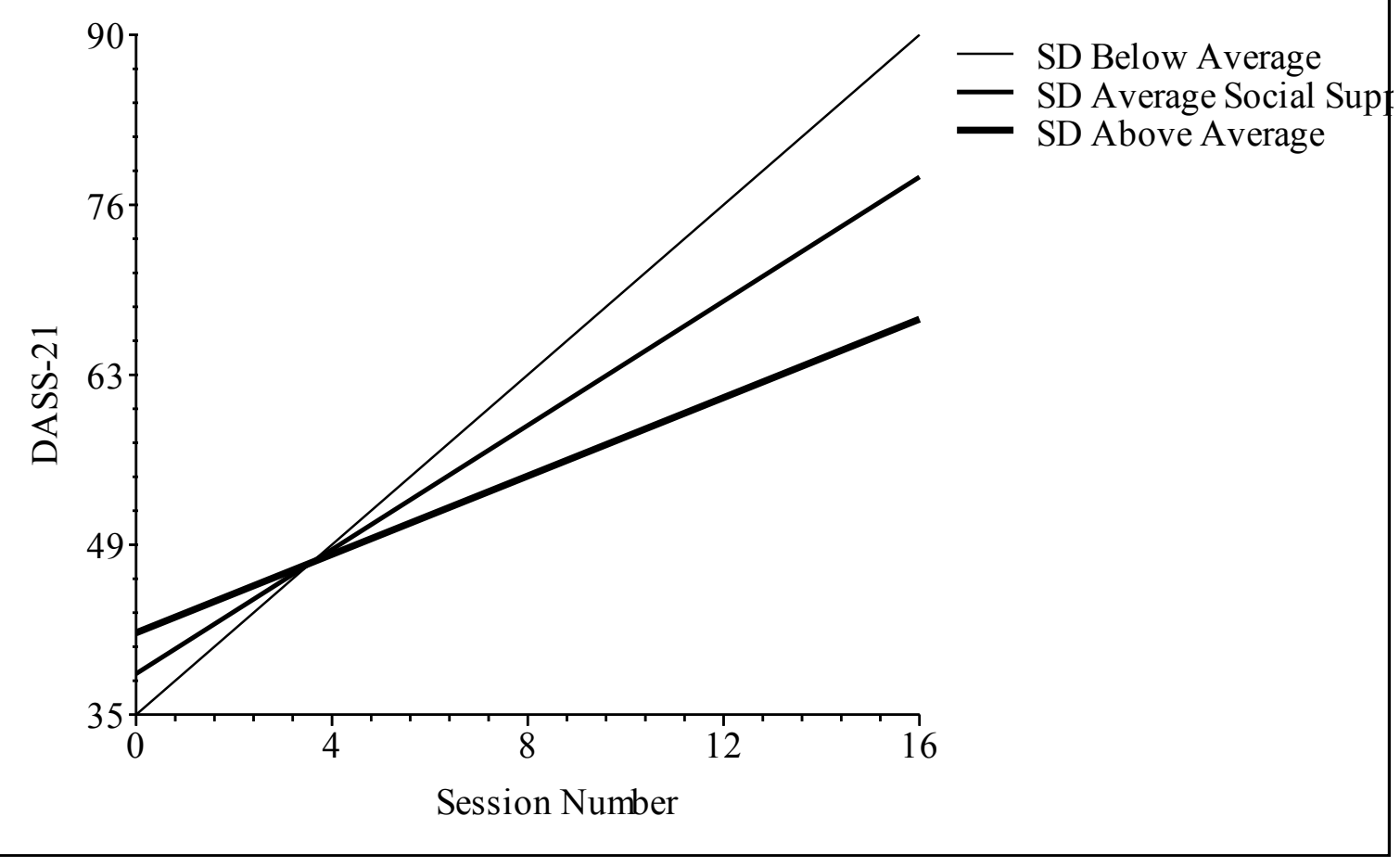

Figure17. DASS-21 trajectories over time in relation to social support.

\section{Hypothesis 2: Therapeutic Alliance and Satisfaction}

For the second hypothesis, it was proposed to examine two factors considered elemental to the psychotherapeutic experience. First, the quality of the therapeutic alliance between patients and their graduate student therapist was evaluated. Second, patients' self-reported 
satisfaction with the quality of psychological services received at PTC was explored.

WAI. As noted above, the WAI was not often provided at regular intervals and score variation from session to session was limited. A $t$-test indicates no significant differences in available scores $(N=19)$ from pre $(M=51.68 ; S D=8.20 ;$ Range $=32-60 ;$ Item Mean $=4.27)$ to post $(M=54.16 ; S D=7.53 ;$ Range $=34-60 ;$ Item Mean $=4.54), t(18)=-1.822, p=.085$, Hedge's $g$ : 0.32). Participants' mean average WAI score, used for the HLM analyses, was 52.57 ( $S D=7.21$; Range $=35-60 ;$ Item Mean $=4.38$ ), which is above the midpoint of 30 and represents $87.62 \%$ of the potential maximum score of 60 . Thus, participants had a positive working alliance with their graduate student psychotherapist. All three factors were highly rated, particularly Goal (representing $91.40 \%$ of the maximum score) and Bond (representing $92.60 \%$ of the maximum score; see table 13). The lowest rated factor was Task (representing $83.05 \%$ of the maximum score), which was still quite high. It appears that participants experienced strong bonds, agreed upon goals, and shared task creation with their graduate student psychotherapist. 
Table 13

Average Ratings for Items on the WAI $(\mathrm{N}=30)$

\begin{tabular}{|c|c|}
\hline Item & $M(S D)$ \\
\hline Goal & $18.28(2.36)$ \\
\hline 4. and I collaborate on setting goals for my therapy. & $4.57(0.78)$ \\
\hline 6. and I are working towards mutually agreed upon goals. & $4.71(0.61)$ \\
\hline 8. and I agree on what is important for me to work on. & $4.61(0.62)$ \\
\hline $\begin{array}{l}\text { 11. and I have established a good understanding of the kind of } \\
\text { changes that would be good for me. }\end{array}$ & $4.39(0.78)$ \\
\hline Task & $16.61(3.08)$ \\
\hline $\begin{array}{l}\text { 1. As a result of these sessions I am clearer as to how I might be able to } \\
\text { change. }\end{array}$ & $3.96(0.82)$ \\
\hline $\begin{array}{l}\text { 2. What I am doing in therapy gives me new ways of looking at my } \\
\text { problem }\end{array}$ & $4.04(0.90)$ \\
\hline $\begin{array}{l}\text { 10. I feel that the things I do in therapy will help me to accomplish the } \\
\text { changes that I want. }\end{array}$ & $4.25(0.95)$ \\
\hline 12. I believe the way we are working with my problem is correct. & $4.36(0.83)$ \\
\hline Bond & $18.52(2.25)$ \\
\hline 3. I believe__ likes me. & $4.46(0.78)$ \\
\hline 5. an I respect each other. & $4.87(0.38)$ \\
\hline 7. I feel that appreciates me. & $4.58(0.70)$ \\
\hline $\begin{array}{l}\text { 9. I feel } \\
\text { approve of. }\end{array}$ & $4.61(0.65)$ \\
\hline Total & $52.99(8.96)$ \\
\hline
\end{tabular}

Note. WAI $=$ Working Alliance Inventory-Short Form. Scores: $1=$ Seldom, $2=$ Sometimes, $3=$ Fairly Often, $4=$ Very Often, and $5=$ Always. Total scores range from 12 to 60 . Based on data from 89 completed WAIs from 30 participants.

CSQ. Similar to the WAI, there was virtually no variability in the CSQ across sessions. It was also not administered reliably at the pre-determined intervals resulting in missing session data. A $t$-test indicates that there were statistically significant differences from pre $(M=28.43$; $S D=3.53)$ to post treatment $(M=29.96 ; S D=3.32), t(22)=-2.71, p=.013$, Hedge's $g=0.45$. However, this may be due the limited variability in scores (the mode rating for all items was 4) and is less clinically useful given there was only a 1.53 point difference in mean CSQ scores from pre to post. The overall average score of $29.74(S D=2.99)$ represents $92.94 \%$, indicating that participants were satisfied with the services they received at the PTC (see Table 14). 
Table 14

Average Ratings for Items on the WAI Groups $(N=30)$

\begin{tabular}{lc}
\hline Item & $M(S D)$ \\
\hline 1. How would you rate the quality of service you received? & $3.87(0.34)$ \\
2. Did you get the kind of service you wanted? & $3.76(0.48)$ \\
3. To what extent has our service met your needs? & $3.47(0.62)$ \\
4. If a friend were in need of similar help, would you recommend our services & $3.81(0.45)$ \\
to him or her? & $3.69(0.64)$ \\
5. How satisfied are you with the amount of help you received? & $3.59(0.59)$ \\
6. Have the services you received helped you to deal more effectively with & \\
your problems? & $3.75(0.46)$ \\
7. In an overall, general sense, how satisfied are you with the service you & \\
received? & $3.80(0.43)$ \\
8. If you were to seek help again, would you come back to our service? & $29.74(2.99)$ \\
Total &
\end{tabular}

Note. WAI $=$ Working Alliance Inventory-Short Form. Ratings range from 1 to 4. Total scores range from 8 to 32 . Based on data from 93 completed CSQs from 30 participants.

\section{Discussion}

Mental illness is highly prevalent in Canada with approximately $20 \%$ of the population living with mental illness each year (Lesage et al., 2006; MHCC, 2012a, 2012b; Smetanin et al., 2011). However, psychotherapy, a well-established treatment for anxiety and depression, is not accessible to most Canadians because of limited government funding and the high cost of private practice (Hunsley, 2002; MHCC, 2012a; Mulvale \& Bourgeault, 2007; Myer \& Payne, 2006; Vasiliadis et al., 2009). One way to improve access is to provide psychotherapy in interprofessional primary care teams, such as FHTs (MHCC, 2012a; OMHLTC, 2011), which appears to be a cost-efficient and effective service delivery model (Cuijpers et al., 2009; Mulvale et al., 2008; Linde et al., 2015; Swenson et al., 2008). However, few FHTs include a clinical psychologist (Cordeiro et al., 2015; Moulding et al., 2009; Mulvale \& Bourgeaut, 2007) and training experiences for graduate students in this emerging area are limited (Bray et al., 2003; Grenier et al., 2008; Masters et al., 2005; Twilling et al., 2000). As well, research on the provision of psychotherapy in an FHT setting is limited (Chomienne at al., 2011; Cordeiro et al., 
2015). The Ryerson University PTC at St. Michael's Hospital's FHTs is a novel mental health care delivery and student training model that provides psychotherapy, predominantly individual CBT, to patients of the FHTs administered by supervised Master's level clinical psychology graduate students at no direct cost to patients. To fill a gap in the literature and study the effectiveness of this model, a pre-post pilot program evaluation was conducted. Specifically, individual CBT provided to patients with a primary diagnosis of a depressive or anxiety disorder, two of the most commonly seen disorders in primary care settings (Archer et al., 2012; Bland et al., 1997; Bray et al., 2003; Chomienne et al., 2011; White, 2008), was examined. Diagnoses and symptom changes were explored, along with the impact of medication use, working alliance, and social support all of which have been previously established as positively impacting mental health outcomes (Bandelow, Seidler-Brandler, Becker, Wedekind, \& Rüther, 2007; Mallinckrodt, 1989; Mann, 2005; Martin et al., 2000; Webb et al., 2011). Client satisfaction was also examined.

\section{Effectiveness of Individual CBT for Anxiety and Depression}

Results from this pilot study suggest that CBT provided by supervised clinical psychology graduate students in an FHT setting is effective for the treatment for depression and anxiety. Participants experienced significant reductions in symptoms related to overall mental health, depression, and anxiety. Eighty percent of participants assessed either no longer met DSM-5 criteria for their primary anxiety or depressive disorder or were in partial remission. In some cases, secondary issues remitted as well. Medication use, working alliance, and social support could not significantly predict these changes, suggesting that these changes are attributable to the treatment provided. Participants largely reported positive working alliance with their graduate student psychotherapists and satisfaction ratings were high overall. These 
findings are similar to those found in other primary care settings (Chomienne at al., 2011;

Cordeiro et al., 2015; Cuijpers et al., 2009; ; Linde et al., 2015; Seekles et al., 2013) and further support the effectiveness of CBT for anxiety and depression (e.g. Hunsley et al., 2014). These findings are particularly striking since treatment was administered solely by novice psychotherapists.

Complex patient population. Participants from this study exemplify the complex and diverse patient population at the PTC. Participants represented a range of ages, cultural backgrounds, sexual orientations, relationship statuses, and educational backgrounds. They experienced a number of life stressors, including challenges with employment, finances, physical health, relationships, the health of others, severe mental health, housing, grief, moving, school, immigration, power of attorney, and dating. Two-thirds had a mental disorder diagnosis prior to treatment at the PTC and slightly more than half had at least one chronic physical illness. Based on the results of a SCID-5-CV assessment, 78\% of participants met DSM-5 criteria for two or more mental disorders, a high rate of comorbidity for a primary care setting (Rodriguez et al., 2004). These comorbidity rates are similar to or higher than those found in other primary care settings (Roca et al., 2009; Rodriguez et al., 2004). Over half of participants had a primary depressive disorder diagnosis while the remaining half had a primary diagnosis of an anxiety disorder, obsessive-compulsive disorder, or post-traumatic stress disorder, with the latter two classified as anxiety disorders in the previous edition of the DSM. Anxiety disorders, followed by depressive disorders, were the most frequent comorbid conditions, though a range of other complex conditions were identified as well, including substance and alcohol use disorders, anorexia and bulimia nervosa, and attention deficit hyperactive disorder.

Individual CBT treatment. CBT fidelity was rated using the CTS. Scores were 
generally above the CTS cut-off, indicating treatment fidelity. On average, clinical psychology graduate students' skill level was good. However, five sessions scored received a score lower than the clinical cut-off. In three of these instances, relationship ruptures or treatment nonadherence were noted. In general, scores obtained were similar to past research involving trainees (e.g. Borkovec et al., 2002; Rakovshik \& McManus, 2010).

The focus of treatment was predominantly anxiety or depression, though in some instances more severe or treatment interfering issues took prominence or were equally targeted in treatment (e.g. alcohol use, bereavement, anorexia nervosa). Participants received between four and 16 sessions of CBT. Those who completed treatment received an average of 12 sessions, while participants who dropped out received an average of six sessions. Treatment duration was similar to or greater than the number of psychotherapy sessions typically provided in primary care settings (Cape et al., 2010; Cordeiro et al., 2015; Cuijpers et al., 2009; Linde et al., 2015; Seekles et al., 2013). In fact, the average number of sessions was more in line with the recommended treatment duration advised for anxiety and depressive disorders (Barlow, 2008; Butler et al., 2006; Cape, Whittington, Buszewicz, Wallace, \& Underwood, 2010; Leahy, Holland, \& McGinn, 2012). Due to the limited number of mental health care providers in FHT settings, it is not always possible for the recommended dose of CBT to be administered. Instead, shorter-term treatments are often provided and there is support for its efficacy (e.g. Cape et al., 2010; Nieuwsma et al., 2012; Roy-Byrne et al., 2010). However, given the range of complexity and symptom severity denoted in this and other FHT studies, it may be beneficial to provide access to both short- and long-term treatment in primary care settings (Blane, Williams, Morrison, Wilson, \& Mercer, 2013), something which the PTC/FHT model provides.

Waitlist. There is some evidence that treatment in primary care teams may be shorter 
than the wait for psychiatric outpatient services (e.g. Kates et al., 2011a). The average time spent waiting from intake to treatment at the PTC was 192 days (approximately half a year), though these times varied widely with some only waiting 73 days (two and a half months) to 310 days (almost one year). At the low end, these wait times were similar to other FHT settings, though the average is much higher (Cordeiro et al., 2015). However, time spent waiting for treatment was similar to other publically funded mental health services and may in fact be shorter than outpatient clinics or other psychiatric services (Anderssen, 2015; Cordeiro et al., 2015). Analyses from this study found no significant differences in OQ-45 or DASS-21 scores between intake and session 1. Thus, participants did not experiences any symptom changes while waiting for treatment.

Diagnostic changes. Eighty-percent of participants who completed a post-treatment SCID-5-CV assessment no longer met full criteria for their primary mental disorder or were in partial remission. There were also instances where participants no longer met criteria for their secondary diagnosis. The remaining participants did not experience a diagnostic change despite receiving a full dose of treatment. Data were not available for those that dropped out of treatment. This study appears relatively unique in its use of a structured diagnostic interview at pre and post treatment. Few published studies examining psychotherapy in primary care utilized standardized diagnostic interviews, instead often relying on clinician expertise, self-report screeners, or clinician administered measures such as the Beck Depression or Anxiety Inventory, Hamilton Depression Rating Scale, and Patient Health Questionnaire (Cape et al., 2010; Cuijpers et al., 2009; Linde et al., 2015; Seekles et al., 2012; Swenson et al., 2008. This is also a strength of the PTC training model that includes training in structured diagnostic interviews, regular use of outcome measures, and other assessments, a key competency area for clinical psychologists 
(Fouad et al., 2009; Krishnamurthy et al., 2004; Rodolfa, et al., 2005).

Overall mental health symptoms. Another strength of this study was the collection of data at multiple time points, not solely before and after treatment. This allowed for changes to be tracked more precisely over time and include information from patients who dropped out. The majority of participants had pre-treatment OQ-45 scores that were above the clinical cut-off, indicating scores were similar to a clinical rather than community population. Participants experienced a statistically significant change in scores from above to below the clinical cut-off by the end of treatment. Half of participants experienced reliable change as indicated by OQ-45 guidelines. Moreover, participants experienced a significant reduction in mental health symptoms over time, further supporting the effectiveness of individual CBT provided in a primary care setting by student trainees. There were no significant differences between those with primary depression versus primary anxiety. Symptom Distress and Interpersonal Relations factors changed significantly from above to below clinical cut-offs by the end of treatment. There were no significant changes in Social Roles scores in relation to the related cut-off. These improvements are similar to and in some cases greater than past research (e.g. higher rates of reliable change, larger effect size) conducted with student learners or in similar environments (Chommienne et al., 2011; Cigrang et al., 2006; Lambert et al., 2002; Nyman, Nafziger, \& Smith, 2010).

Symptoms of depression and anxiety. At intake, the majority of participants' Depression scores on the DASS-21 fell in the moderate to extremely severe range. Participants experienced a significant reduction in symptom severity from pre to post treatment on this factor. Only $50 \%$ of participants' Anxiety scores fell in the same severity range and did not change significantly from pre to post. Over the course of individual CBT, participants' total DASS-21 
scores reduced significantly over time. There were no significant differences in symptom changes over time between patients with a primary diagnosis of an anxiety disorder versus depressive disorder. DASS-21 changes from pre to post were similar to past research, though some previous studies documented a greater reduction of overall symptoms (Bradbury et al., 2008; Dear et al., 2011; Titov et al., 2010; Troeung, Egan, \& Gasson, 2014), which may be related to the lack of significant change in severity for the Anxiety and Stress scales. Nonetheless, this further supports the effectiveness of individual CBT provided in a primary care and student training setting in reducing symptoms of depression and anxiety.

Medication use, working alliance, and social support. Medication use, working alliance, and social support have previously been documented as having an impact on treatment outcomes. Medication is a well-established treatment for anxiety and depression (e.g. Bandelow et al., 2007; Mann, 2005). It is also the most common treatment administered to patients with these issues (Roberge et al., 2011; Roberge et al., 2014). Participants of this study were not excluded for taking medication and in fact three-quarters of participants were taking medication for mental health purposes at the time of intake. This proportion was similar to, though slightly higher than previously documented medication use in primary care settings (Roberge et al., 2014). Patients receiving individual CBT and taking medication experienced faster rates of symptom reduction than patients not taking medication, though this difference was not statistically significant.

Working alliance, particularly strong alliance, has been found to contribute to outcome changes (Martin et al., 2000; Webb et al., 2011). Participants reported having good working alliance with their graduate student psychotherapists and achieved scores similar to past research (e.g. Webb et al., 2011). Similar to medication use, although higher scores on the WAI were 
related to faster symptom reduction, this was not a statistically significant difference.

Social support has also been shown contribute to improvements in mental health (Mallinckrodt, 1989). Almost all participants identified having at least some social support. Those with no social support experienced increased symptomatology over time, whereas those with social support experienced symptom reduction over time. However, this difference was not statistically significant. Moreover, the HLM analysis could not compute robust standard error and therefore these results should be interpreted with caution. It will be important in the future to research more nuanced differences in social support rather than its general presence or absence. In sum, contrary to what was expected, medication use, working alliance, and social support could not predict outcome changes. Thus, the results can likely be attributed to the treatment itself.

Satisfaction. Overall, participants reported being satisfied with the psychological services provided at the PTC throughout their treatment. They indicated satisfaction with the quality of services, would recommend the services to a friend, and would return for services if need be. In general, participants received the services they were looking for, their needs were met, and they were satisfied with the amount of treatment they received. These high ratings are similar to recent research examining the impact of providing psychotherapy in a FHT setting (Chomienne et al., 2011; Cordeiro et al., 2015) and further supports patients satisfaction with and support of Psychology in FHTs.

\section{Limitations and Future Directions}

Due to the real-world nature of this study, a number of challenges arose. First and foremost is the small sample size and related low power, particularly in detecting the influence of predictor variables on treatment outcome. This study was framed as a pilot study due to its size, 
specific focus (i.e. primary anxiety and depression), and goal of gathering preliminary evidence on the effectiveness of individual CBT provided at the PTC. Although the sample satisfied requirements for statistical analyses and large effect sizes were found, replication on a larger scale is needed. It will also be beneficial to compare similarities and differences between different FHTs that deliver psychotherapy, potentially through a multi-site study.

Data were only collected from pre to post treatment. It will be important to track outcomes beyond treatment completion to examine whether patients experience lasting change.

Future research should also examine individual CBT with a comparison or waitlist control group. Although this study did not have a comparison group per se, participants' time on the waitlist acted as a de facto comparison group and no significant differences in symptomatology from intake to the start of treatment were found. A comparison group was initially proposed for this study for which patients would come to the PTC for symptom monitoring every three weeks for 10 weeks. However, it became clear this was not feasible. Patients were unavailable to return to the PTC at regular intervals without treatment and the lack of a researcher regularly onsite also presented a challenge. Future research may want to consider online or mail-based symptom monitoring or employing an onsite research assistant to engage participants in program evaluation efforts.

There were a number of logistical and practical challenges that arose as part of the natural changes that occur at a dynamic work environment such as the PTC and FHTs. During the course of this study, there were changes in personnel and graduate student cohorts, new DSM and SCID editions, and scheduling changes. The implementation of the standardized procedures and measures alone posed numerous challenges, and understandably so. These changes take a great deal of time, resources, and invisible work to implement and there are often competing 
demands (Flottorp, Håvelsrud, \& Oxman, 2003; Richter-Sundberg, Nyström, Krakau, \&

Sandahl, 2015). In retrospect, it would have been beneficial to conduct an implementation study prior to the pilot study. These challenges resulted in delays at various phases of the program evaluation and data was collected for fewer participants than proposed. Nonetheless, all parties involved worked diligently to problem solve these barriers in order to implement the program evaluation (Kirchner et al., 2010) and the resulting study is an admirable first step in documenting the effectiveness of CBT provided in an FHT setting.

Lastly, the PTC is a unique service delivery and treatment model. To this author's knowledge, the PTC is the only training facility integrated fully into a set of FHTs in Canada. Though data from this study adds to the growing body of research documenting the benefits of including Psychology in the FHT teams, it may be difficult to generalize these findings to other settings.

\section{Implications and Conclusions}

Results of this study are hopeful. The findings indicate that individual CBT for anxiety and depression provided by clinical psychology graduate students in an FHT setting is effective irrespective of medication use, working alliance, and potentially social support. Participants reported strong working alliance with their student psychotherapists and satisfaction with services overall. This provides further evidence in support of the inclusion of Psychology in FHTs and other interprofessional primary care models. As FHTs continue to grow in numbers, FHT executive directors may want to consider the inclusion of a psychologist or even partnering with clinical psychology training facilities as a novel way to improve access to clinicians and share resources. This has been echoed by recent mental health strategies that advocate for greater access to psychotherapy through interprofessional primary health care teams (MHCC, 
2012a; OMHLTC, 2011). Physicians regularly refer to psychologists (Roberge et al., 2014) and have expressed a desire to work more collaboratively with them in order to improve the quality of mental health treatment in primary care settings (Chomienne et al., 2011; Cohen \& Peachey, 2014; Peachey et al., 2013). Reports from primary care teams that include a psychologist indicate physicians appreciate having them on the team and are satisfied with their services (Cordiero et al., 2015). Psychologists themselves acknowledge the need for improved access to their services and are open to different compensation packages (Bradley \& Drapeau, 2014). Furthermore, the inclusion of psychologists in FHTs has the potential to remove two of the greatest barriers to mental health treatment by increasing access to and availability of psychologists who can provide CBT, an evidence-based treatment for a range of mental disorders (Gagné, 2005; Kirby \& Keon, 2006). And training graduate students in this area can ensure that future clinical psychologists develop the expertise in working in this relatively new area of psychology. 
STUDY 2: PERSPECTIVES ON PATIENT CARE, INTERPROFESSIONALISM, AND

STUDENT TRAINING AT THE PTC

\section{Study Design}

\section{Concurrent Triangulation Design Model}

To examine the integration of the PTC into the St. Michael's Hospital's FHTs, a mixed methods approach was undertaken, comprised of two phases, one quantitative and one qualitative. More specifically, this study employed a concurrent triangulation explanatory design, meaning that both phases were exploratory in nature and conducted at the same time (Creswell et al., 2004; Creswell, 2009). As with a typical triangulation design, the quantitative and qualitative data are reported separately and then reviewed jointly in the discussion section (Creswell et al., 2004).

\section{Ethical Considerations}

Ethical approval and permissions. This study was reviewed and approved by the St. Michael's Hospital Research Ethics Board (received October 16, 2015) and Ryerson University Research Ethics Board (received November 18, 2015) prior to the start of data collection. Permission was obtained and fees were paid (where required) for all measures and materials used in this study.

Informed consent. In the informed consent, the study was described in detail and potential risks and benefits were outlined (see Appendix J). Participants were told that participation was voluntary and that they could withdraw their consent at any time or decline to answer any question without penalty. They were informed that data collected would be kept confidential and all electronic data encrypted and password protected. Participant data was anonymized and only aggregate data was analyzed. Identifying information was not collected 
(e.g. name, birth date). Lastly, contact information for the researchers and the St. Michael's Hospital Research Ethics Board was provided.

For Phase 1, information on Qualtrics, the online web survey company, was also provided. Participants were made aware that as a company from the United States (US), it is subject to US laws and therefore there is a chance that information may be unintentionally released. Participants indicated their consent by clicking, "Yes I choose to participate-CLICK HERE" to continue or "No, I choose note to participate-PLEASE CLOSE THE WINDOW," which ended the survey.

For Phase 2, participants were given a written informed consent to review and sign. They were reminded that participation was voluntary and were welcomed to ask any questions.

Written debriefing. At the end of each study, participants were provided a written debriefing online (Phase 1) or hard copy version in person (Phase 2) (see Appendix K). The written debriefing provided reviewed background information on the PTC and the study, as well as contact information for the researchers and Ryerson University and St. Michael's Hospital Research Ethics Boards. Participants were also thanked for their participation.

\section{Compensation.}

Phase 1. As incentive to participate, individuals who completed the online questionnaire for Phase 1 were offered the option to be entered into a draw to win one of four $\$ 50$ Chapters/Indigo gift cards (two for the clinical psychology students, one for health care providers, and one for patient participants). In order to protect anonymity, participants who chose to enter their name in the draw were directed to separate survey that was unconnected to their online questionnaire data to enter their contact information and participant type. The four winners were provided the gift card in person or by mail, depending on the participant's 
preference.

Phase 2. As an incentive to participate in one-on-one interviews, participants were provided with two TTC tokens for transportation, light refreshments, and a \$5 Tim Hortons gift card.

Data storage. All data were anonymized and includes no identifying information. The exception to this is contact information for participants who consented to have their name entered into a draw for Phase 1 and contact information for interviewees in Phase 2. This information was destroyed after winners were chosen for Phase 1 and after all interviews took place for Phase 2. All data are stored on secure servers, electronic files are encrypted and password protected, and all paper documents (e.g. informed consents for Phase 2) are stored in a locked facility at Ryerson University. The de-identified data will be kept for 10 years per the regulations from the Canadian Psychological Association Ethical Guidelines. Informed consents will be kept separate from participant data. For Phase 2 interviews, audio-recordings were destroyed after transcription. In the transcriptions, all individuals were identified as 'participant' and identifying information was removed from quotations included in this document. Only the researchers and trained research assistants associated with the Community-Engaged Research in Culture and Health lab have access to the evaluation data.

Qualtrics. Qualtrics is a web survey service housed in the United States. It meets the Health Insurance Portability and Accountability Act health care privacy record standards and is housed on secure servers. All Qualtrics accounts are password protected. In the informed consent, participants were made aware that as a US-based company, Qualtrics is subject to the Patriot Act and as such, their data may be compromised, though the likelihood of this occurring is low. 
Other ethical considerations. The principal researcher is a current clinical psychology graduate student at Ryerson University who completed a year-long practicum at the PTC. As such, there were a number of ethical considerations. To protect anonymity, for the electronic questionnaire, participants were not required to provide their name or demographic information that could lead to their identification (e.g. age, year of study). A trained research assistant with no connection to the PTC conducted all but one interview due to an ongoing relationship with the interviewee. This was conducted by the principal investigator who did not have a previous or current relationship with the interviewee.

Data analysis strategy. Given the exploratory nature of this study, descriptive statistics comprise the majority of the results for Phase 1. Where applicable, $t$-tests and ANOVAs were employed and effect sizes calculated when comparing groups. For Phase 2, a thematic analysis was used to identify codes within the data (see below for more detail).

\section{Phase 1 Online Questionnaire: Method}

\section{Participants}

Thirty-nine participants took part in the online study, including 25 clinical psychology graduate students (64\%), 12 health care providers (31\%), and two patients (5\%) (see Table 15 ). One additional person gave consent then subsequently did not view or complete any other portion of the survey. Seven participants did not complete one or more of the measures in the survey. Of the students, 18 were past practicum students and 7 were being trained at the PTC at the time of data collection. The health care providers were from Medicine, Social Work, and Chiropractic $(N=12)$, the majority of whom were from medicine. Only three of the health care providers were collocated at the same clinic as the PTC (25\%). Of the patients, one patient (50\%) was a past patient of the PTC, while one was a current patient (50\%). Both patients 
indicated that their primary care physician was collocated at the PTC $(N=2 ; 100 \%)$. Both had also received assessment at the PTC, while only one patient participant had received individual psychotherapy; neither had received group psychotherapy at the PTC.

Table 15

Demographic Information for Clinical Psychology Graduate Student, Patient, and Health Care Provider Participants for Online Questionnaire

\begin{tabular}{ccc}
$\begin{array}{c}\text { Graduate } \\
\text { Students } \\
(N=25)\end{array}$ & $\begin{array}{c}\text { Health Care } \\
\text { Provider } \\
(N=12)\end{array}$ & $\begin{array}{c}\text { Patients } \\
(N=2)\end{array}$ \\
\hline$n(\%)$ & $n(\%)$ & $n(\%)$
\end{tabular}

\begin{tabular}{llccc}
\hline Gender & & & & \\
& Female & $23(92 \%)$ & $8(67 \%)$ & $1(50 \%)$ \\
Age & Male & $2(8 \%)$ & $4(33 \%)$ & $1(50 \%)$ \\
& $18-25$ & $7(28 \%)$ & - & - \\
& $26-30$ & $14(56 \%)$ & - & $1(50 \%)$ \\
& $31-40$ & $2(8 \%)$ & $6(50 \%)$ & - \\
& $41-50$ & $2(8 \%)$ & $2(17 \%)$ & $1(50 \%)$ \\
& $51-60$ & - & $2(17 \%)$ & - \\
& $61-70$ & - & $2(17 \%)$ & - \\
Cultural & & & & \\
Background & Asian/African/Black/Caribbean/Other & $1(4 \%)$ & $4(17 \%)$ & - \\
& White/European & $24(96 \%)$ & $8(67 \%)$ & $2(100 \%)$ \\
Highest & & & & \\
Level of & Bachelor's Degree & $8(32 \%)$ & - & $2(100 \%)$ \\
Education & Master's Degree & & & - \\
Completed & Professional Degree & $17(68 \%)$ & $2(17 \%)$ & - \\
& Doctoral Degree & - & $9(75 \%)$ & - \\
\hline
\end{tabular}

Note. Total percentages may not equal 100 as a result of rounding.

\section{Materials}

\section{Attitudes Toward Health Care Teams Scale (ATHCT; Heinemann, Schmitt, Farrell,}

\& Brallier, 1999; Hyer, Fairchild, Abraham, Mezey, \& Fulmer, 2000). This reliable and validated self-report measure (see Heinemann et al., 1999; Hyer et al., 2000) has been used in a variety of interdisciplinary, health care team environments, including St. Michael's Hospital (see 
Kenaszchuk, Reeves, Nicholas, \& Zwarenstein, 2010; Kenaszchuk, MacMillan, van Soeren, \& Reeves, 2011). The 21-item, three-factor (Attitudes Toward Team Value, Attitudes Toward Team Efficiency, and Attitudes Toward Physician Shared Role) version of this measure was used (Fulmer et al., 2005; Kenaszchuk et al., 2010, 2011; Leipzig et al., 2002). Examples of items from the ATHCT, which are rated on a six-point Likert scale from "Strongly Agree" to "Strongly Disagree," are as follows; scores range from 0 to 105 with higher scores indicating more positive beliefs about interprofessional teamwork (Leipzig et al., 2002). To gauge Attitudes Toward Team Value (11 items), items include, "The team approach improves the quality of care to patients" and "Patients receiving team care are more likely than other patients to be treated as whole persons." To explore Attitudes Toward Team Efficiency (five items), statements include, "Working in teams unnecessarily complicates things most of the time" and "Patients are less satisfied with their care when it is provided by a team." Statements for the last factor, Attitudes Toward Physician Shared Role (five items), include, "Physicians are natural team leaders" and "The physician should not always have the final word in decisions made by health care teams." Nine of the items are reverse coded (e.g. items $1,4,6,8,10,12,15,16,18$ ). See Appendix L for more detail. This self-report takes approximately seven to ten minutes to complete. Data from the ATHCT appeared to be highly reliable $(\alpha=.80)$. However, the subscales varied in reliability: Attitudes Toward Team Value ( $\alpha=.84)$, Attitudes Toward Team Efficiency $(\alpha=.63)$, and Attitudes Toward Physician Shared Role $(\alpha=.72)$.

\section{Interprofessional Collaboration Scale (ICS; Kenaszchuk, Reeves, Nicholas, \&}

Zwarenstein, 2010). The ICS was adapted from a nursing questionnaire (see Adams, Bond, Arber, 1995; Lake, 2002) and created to measure interprofessional collaboration beyond traditional physician-nurse teams for use with a range of health care professionals in acute care 
settings, including primary care clinics (Kenaszchuck et al., 2010, 2011). Initial studies have found this self-report measure to be a reliable and valid measure that differs from the constructs tapped into by the ATHCT (Kenaszchuck et al., 2010, 2011). Three factors comprise the ICS (Communication, Accommodation, and Isolation), and includes 13 items scored on a four-point Likert scale ranging from "Strongly Disagree" to "Strongly Agree" (see Appendix M). Five items are reverse coded: item 3 , item 8 , item 11 , item 12 , and item 13 . Statements for the Communication factor (five items) include, "The team has a good understanding about their respective responsibilities" and "Team members anticipate when they will need others' help." The Accommodation factor (five items) includes statements such as, "Team members are willing to discuss individuals' issues" and "Team members would be willing to cooperate with new, agreed upon practices." The third factor, Isolation (three items), is comprised of items such as, "Some individuals would not be willing to discuss new practices with other team members." This measure takes approximately five minutes to complete. A reliability analysis indicated data for the ICS overall were reliable $(\alpha=.79)$, as were the subscales: Communication $(\alpha=.71)$, Accommodation $(\alpha=.68$.$) , and Isolation (\alpha=.62)$.

\section{Interdisciplinary Education Perception Scale (IEPS; Luecht, Madsen, Taugher, \&}

Petterson, 1990). The IEPS examines differing perceptions of a range of health care professionals, including physicians, social workers, chiropractors, pharmacists, and nursing, in relation to interdisciplinary collaboration and training (Hawk et al., 2002; Luecht et al., 1990; Goellen, De Clercq, Huyghens, \& Kerckhofs, 2006). This self-report measure allows health care professionals to evaluate how they view their own profession, as well as the profession of other interdisciplinary team members (Mu, Chao, Jensen, \& Royeen, 2004). The IEPS consists of four subscales (Competence and Autonomy, Understanding Others' Value, Perceived Need for 
Cooperation, and Perception of Actual Cooperation) comprised of 18 items that are rated on a six-point Likert scale where 1 indicates "Strongly Disagree" and 6 indicates "Strongly Agree" (Hawk et al., 2002; Goellen et al., 2006; Luecht et al., 1990; Neill, Hayward, \& Peterson, 2007). The Competence and Autonomy factor includes eight statements such as, "Individuals in my profession are well trained" and "Individuals in other professions respect the work done by my profession" (Luecht et al., 1990; Neill et al., 2007). Understanding Others' Value includes three statements such as, "Individuals in my profession have a higher status than individuals in other professions." The third factor, Perceived Need for Cooperation, is comprised of two statements including, "Individuals in my profession need to cooperate with other professions." The final factor, Perception of Actual Cooperation, consists of five items such as, "Individuals in my profession have good relations with people in other professions" and "Individuals in my profession are willing to share information and resources with other professionals." The measure takes approximately five to seven minutes to complete (see Appendix N).

A reliability analysis for the IEPS indicates that it is reliable $(\alpha=.81)$. However, results for the four factors were mixed; the Competence and Autonomy $(\alpha=.79)$ and Perception of Actual Cooperation $(\alpha=.76)$ factors were highly reliable, whereas the Perceived Need for Cooperation $(\alpha=.28)$ and Understanding Others' Value $(\alpha=.42)$ factors were not. This may be due to inconsistent factors structures that a recent article argues changes based on the sample demographics and therefore, the original four factor structure is not reliable (Vaughan, Macfarlane, Dentry, \& Mendoza, 2014). Because of the different factor structures and manner in which the data are presented across other studies, the item mean is included and data for Perceived Need for Cooperation and Understanding Others' Value are not reported. 


\title{
Self-efficacy for Interprofessional Experiential Learning Revised (SEIEL-R; Mann,
}

\author{
McFetridge-Durdle, Breau, Clovis, Martin-Misener, Matheson, Beanlands, \& Sarria,
}

2012). The SEIEL is a new self-report measure developed for a program called Seamless Care:

An Interprofessional Education Project for Innovative Team-Based Transition Care to measure students' self-efficacy and ability to fulfill their role in an interdisciplinary learning environment (Mann et al., 2012; Seamless Care, 2008). The original measure is comprised of 16 items and two factors, Interprofessional Interaction and Interprofessional Team Evaluation and Feedback, which is rated on a scale from one to ten, where 1 indicates "Very Low Confidence" and 10 indicates "Very High Confidence" (Mann et al., 2012; Seamless Care, 2008). However, given the unique training experience at the PTC, this scale was modified; some words were omitted (e.g. Ryerson students do not currently train with other students, but rather other professionals) and four items were dropped to create the SEIEL-R (see Appendix O). Statements for the SEIEL-R include, "Working with different professions to resolve problems in the team," "Working with different professionals to understand our respective roles in an interprofessional team," and "Interacting with professionals and disciplines different from my own." This selfreport takes about three-to-five minutes to complete.

\section{Client Satisfaction Questionnaire-8 (CSQ-8; Larsen, Attkisson, Hargreaves, \&}

Nguyen, 1979; Attkisson \& Zwick, 1982; Attkisson \& Greenfield, 2004). The CSQ-8 is a reliable and valid measure that has been used across a number of different programs and institutions that provide psychotherapy to assess client satisfaction (Attkisson \& Zwick, 1982; Attkisson \& Greenfield, 2004). This self-report is comprised of eight items that are rated on a four-point Likert scale and takes approximately three to five minutes to complete (see Appendix E). It includes items such as, "How would you rate the quality of service you have received?," 
with responses ranging from "Excellent" to "Poor," and "In an overall, general sense, how satisfied are you with the service you have received?," with responses ranging from "Very Satisfied" to "Quite Dissatisfied." This measure takes approximately one to three minutes to complete.

Contact with Interprofessional Team Measure (CITM). This brief self-report measure was generated to document the frequency with which patients and health care providers interact with other health care providers within the FHTs. The health care provider version is comprised of four items (see Appendix P). For the first two questions, health care providers are asked to rate the frequency with which they receive and provide consultation to FHT health care professionals outside of their field on a scale from one to seven, where 1 indicates "Never" and 7 indicates "Almost Always." The remaining two questions inquire about the percentage of patient cases for which they receive or provide consultation to a range of health care professionals, including students, at the FHTs (e.g. social worker, physician, chiropractor, nurse, etc.). For example, participants are asked, "In the past year, approximately what percentage of patient cases did you provide consultation to another health care professional/student within the Family Health Teams? (Does not have to equal 100\%)." The patient version of this questionnaire comprises three questions (see Appendix Q). To start, patients are asked, "How many different health care professionals have been involved in your patient care at St. Michael's Hospital Family Health Teams (e.g. physician, nurse, nurse practitioner, chiropractor, social worker, clinical psychology student, dietitian, pharmacist, etc.)?" Next, they are asked to rate, "In the past year, how often did you receive care from more than one health care professional from St. Michael's Hospital Family Health Teams?," using a scale from one to five, where 1 indicates "Never" and 5 indicates "Very Often." Lastly, patients are asked to indicate the types of health 
care professionals involved in their care at the FHT in the past year.

Demographics. This brief survey was created to gather general, non-specific demographic information. Participants were asked to identify their participant group and based on this, were directed to a specific set of demographic questions: patients (seven items), health care providers (six items), and clinical psychology graduate students (five items; see Appendix R). All participant versions included items asking participants to choose their age range (e.g. 1825, 26-30, 31-40), gender (e.g. male, female, intersex, transgender), cultural background/ethnic origin (e.g. Aboriginal, Asian/Island Pacific, Black/African/Caribbean, Mixed-Ethnicity, White/European), and highest level of education completed, including some secondary (high) school, no diploma; college, CEGEP, or registered apprenticeship certificate/diploma; bachelor's degree; and professional degree (e.g. MD, LLB). Students and patients were asked to indicate whether they were a past or present student or patient of the PTC, respectively. Patients and health care providers were asked about whether their home FHT was collocated with the PTC, such as "Is your family physician co-located at the same clinic as the Psychology Training Clinic" with responses including "Yes-Psychology Training Clinic is on site (i.e. Health Centre at 80 Bond)," "No-my physician is at a different site," and "I don't know." Patient participants were also asked to check all of the services they received at the PTC, including assessment, individual psychotherapy, and group psychotherapy. Lastly, the health care provider version included an item asking participants to indicate their position at the St. Michael's Hospital FHTs, such as Chiropractor, Nurse, Physician, Dietitian, Psychiatrist, or Social Worker. This survey takes approximately two to five minutes to complete.

\section{Procedure}

Recruitment. Recruitment and data collection took place between February and July 
2015. Three participant groups were recruited for Phase 1:1) past or present patients of the PTC; 2) health care providers at St. Michael's Hospital FHTs, including physicians, nurses, nurse practitioners, and allied health practitioners (e.g. social worker, dietitian, chiropractor, pharmacist); and 3) past or present Ryerson University clinical psychology graduate students who trained at the PTC. Patients were referred to the study by health care providers and graduate students at the PTC, as well as flyers in the FHT waiting rooms. Health care providers and clinical psychology graduate students were recruited through St. Michael's Hospital internal email by administrative staff not associated with the study. Flyers were also placed in staff mailrooms and seating areas (e.g. computer room, lunch room). Materials used to recruit participants included an overview of the study, potential compensation (i.e. a draw for a gift card), a web link to access the study, and researchers contact information.

Online questionnaire. The web link brought participants to the Qualtrics generated online questionnaire. At the start of the electronic questionnaire, participants were directed to the informed consent. Participants had to click the option that they consented to participate in order to move the questionnaire forward; if participants declined consent, they were thanked for their time and the survey closed. Participants then identified whether they were a patient, health care provider, or clinical psychology graduate student, at which time they were directed to a set of questionnaires tailored to the group they chose.

To start, all participants were directed to their specific version of the demographic questionnaire. Patients were directed to the ATHCT, CITM-Patient Version, and CSQ-8. Graduate students were directed to the following questionnaires: ATHCT, CITM-Health Care Provider Version, ICS, IEPS, and SEIEL-R. Health care providers were directed to the ATHCT, CITM-Health Care Provider Version, ICS, and IEPS. After the questionnaires, participants were 
provided a written debriefing and thanked for their participation. Lastly, they were asked about their interest in participating in the Phase 2 interviews and whether they would like to have their name entered their name into a draw to win a $\$ 50$ Chapters/Indigo gift card. Those who chose to enter their name in the draw or participate in an interview were directed to a survey separate from their participant data, where they provided their participant type and contact information. All participants completed the survey within one day of initially logging onto the study (Mean= 53 minutes; Median $=9$ minutes; $S D=230$ minutes). The range is high due to two outliers, participants who completed their survey over several hours; when these outliers are removed, the range is between five and 21 minutes. Names of participants who consented to the draw were entered into one of three draws for each participant group. Winners were chosen at random, contacted, and prizes were awarded. Results for the CSQ-8 were not tabulated for confidentiality reasons because only one patient participant completed this measure.

\section{Phase 1 Online Questionnaire: Results}

\section{Interprofessional Patient Care}

Attitudes Toward Health Care Teams. Overall, it appears that participants hold positive beliefs about interprofessional teamwork with an average of $75.03(S D=10.83)$ out of a possible score of 105 . Yet, this average score represents only $71.66 \%$ of the potential maximum score. These totals varied by participant group with relatively similar scores for clinical psychology graduate students $(M=74.80, S D=11.85)$ and health care providers $(M=74.10$, $S D=8.96)$, while patient scores were higher $(M=83.00, S D=5.66)$. However, with only two patient participants, findings should be interpreted with caution. No significant differences were found between groups, $F(2,35)=0.57, p=.568, r=0.18$. 
With regards to the ATHCT factors, scores were also moderately high: Attitudes Toward Team Values $(M=40.92, S D=7.29)$; Attitudes Toward Team Efficiency $(M=18.34, S D=3.50)$; and Attitudes Toward Physician Shared Role $(M=15.76, S D=4.69)$. For the Attitudes Toward Team Values factor, no significant differences were found between groups, $F(2,35)=0.98$, $p=.385, r=0.23$ (see Table 16 for more detail). No between group differences were found for the Attitudes Toward Team Efficiency factor either, $F(2,35)=1.70, p=.197, r=0.30$. The Attitudes Toward Physician Shared Role factor approached statistical significance, $F(2,35)=2.88, p=.070$, $r=0.38$. Examined more closely, clinical psychology graduate students had significantly higher scores than health care providers, $t(34)=-2.35, p=.025$, Hedge's $g=0.85$. There were no significant differences regarding the patient group.

No group norms exist for the ATHCT or its factors. Moreover, there are at least two other versions of the measure, altered in part to differing factor structures (Heinemann, Schmitt, Farrell, \& Brallier, 1999) and reduced item numbers (Curran, Sharpe, \& Forristall, 2007) making comparisons between studies difficult. Compared to studies that employed the same version of the ATHCT, total and factor scores from this study were generally lower than most (e.g. Giordano et al., 2013; Kenaszchuk et al., 2011; Leipzig et al., 2002), but higher than others (e.g. Robbens et al., 2012). Scores were similar to baseline scores found in pre-post studies examining changes in the ATHCT after the implementation of an interprofessional training intervention (e.g. Fulmer et al., 2005; Giordano et al., 2013). As well, the lack of differences between groups of different disciplines is atypical; often there are differences between health care professionals, particularly when compared to physicians (Giordano et al., 2013; Kenaszchuk et al., 2011; Leipzig et al., 2002). Several studies found that physicians had lower ATHCT scores than other professions, scores that were similar to those found in the health care provider 
group which was comprised mostly of physicians (Fulmer et al., 2005; Giordano et al., 2013; Kenaszchuk et al., 2011; Leipzig et al., 2002). Lastly, it is important to note that there are few published studies examining levels of interprofessionalism with psychologists or psychology students and those that are available are limited in nature. For example, of the studies noted above, only Giordano and colleagues (2013) included students training in couple and family therapy, of which there were only six participants. 
Table 16

Comparison of ATHCT Factors and Scores Between Participant Groups

\begin{tabular}{|c|c|c|c|}
\hline Item & $\begin{array}{l}\text { Graduate } \\
\text { Students } \\
N=25 \\
M(S D)\end{array}$ & $\begin{array}{l}\text { Health Care } \\
\text { Providers } \\
N=12 \\
M(S D)\end{array}$ & $\begin{array}{l}\text { Patients } \\
\qquad \begin{array}{l}N=2 \\
M(S D)\end{array}\end{array}$ \\
\hline Attitudes Toward Team Value & $40.16(7.92)$ & $41.45(5.77)$ & $47.50(4.95)$ \\
\hline $\begin{array}{l}\text { 2. The team approach improves the quality of care to } \\
\text { patients }\end{array}$ & $4.44(0.71)$ & $4.55(0.69)$ & $5.00(0.00)$ \\
\hline $\begin{array}{l}\text { 3. Team meetings foster communication among team } \\
\text { members from difference disciplines }\end{array}$ & $4.04(1.10)$ & $4.36(0.92)$ & $5.00(0.00)$ \\
\hline $\begin{array}{l}\text { 5. Patients receiving team care are more likely than } \\
\text { other patients to be treated as whole persons }\end{array}$ & $3.32(1.31)$ & $3.36(1.12)$ & $5.00(0.00)$ \\
\hline $\begin{array}{l}\text { 7. Working on a team keeps most health professionals } \\
\text { enthusiastic and interested in their job }\end{array}$ & $2.88(0.97)$ & $4.27(0.79)$ & $3.00(1.41)$ \\
\hline $\begin{array}{l}\text { 9. Developing a patient care plan with other team } \\
\text { members avoids errors in delivery care }\end{array}$ & $3.40(1.26)$ & $3.27(1.49)$ & $4.50(0.71)$ \\
\hline $\begin{array}{l}\text { 11. Health professionals working on teams are more } \\
\text { responsive than others to the emotional and financial } \\
\text { needs of patients }\end{array}$ & $2.96(1.21)$ & $2.36(1.43)$ & $3.00(1.41)$ \\
\hline $\begin{array}{l}\text { 14. The give and take among team members help them } \\
\text { make better patient care decisions }\end{array}$ & $4.20(0.82)$ & $4.00(0.78)$ & $5.00(0.00)$ \\
\hline $\begin{array}{l}\text { 17. Hospital patients who receive team care are better } \\
\text { prepared for discharge than other patients }\end{array}$ & $3.80(1.04)$ & $3.91(0.94)$ & $4.00(1.41)$ \\
\hline $\begin{array}{l}\text { 19. The team approach makes the delivery of care more } \\
\text { efficient }\end{array}$ & $3.40(1.19)$ & $3.64(0.92)$ & $5.50(0.71)$ \\
\hline $\begin{array}{l}\text { 20. The team approach permits health professionals to } \\
\text { meet the needs of family caregivers as well as patients }\end{array}$ & $3.52(0.87)$ & $3.82(0.75)$ & $3.50(0.71)$ \\
\hline $\begin{array}{l}\text { 21. Having to report observations to the team helps team } \\
\text { members better understand the work of other health } \\
\text { professionals }\end{array}$ & $4.20(1.16)$ & $3.91(0.75)$ & $5.00(0.00)$ \\
\hline Attitudes Toward Team Efficiency & $17.64(3.65)$ & $19.45(2.73)$ & $21.00(4.24)$ \\
\hline $\begin{array}{l}\text { 1. Working in teams unnecessarily complicates things } \\
\text { most of the time } \dagger\end{array}$ & $4.04(1.02)$ & $4.45(0.69)$ & $5.00(0.00)$ \\
\hline $\begin{array}{l}\text { 8. Patients are less satisfied with their care when it is } \\
\text { provided by a team } \dagger\end{array}$ & $3.72(1.02)$ & $4.36(0.81)$ & $3.00(2.83)$ \\
\hline $\begin{array}{l}\text { 10. When developing interdisciplinary patient care } \\
\text { plans, much time is wasted translating jargon from other } \\
\text { disciplines } \dagger\end{array}$ & $3.28(0.98)$ & $3.82(0.98)$ & $5.00(0.00)$ \\
\hline $\begin{array}{l}\text { 12. Developing an interdisciplinary patient care plan is } \\
\text { excessively time consuming } \dagger\end{array}$ & $3.40(1.32)$ & $3.27(0.91)$ & $5.00(0.00)$ \\
\hline $\begin{array}{l}\text { 15. In most instances, the time required for team } \\
\text { meetings could be better spent in other ways } \dagger\end{array}$ & $3.20(1.29)$ & $3.55(0.93)$ & $3.00(1.41)$ \\
\hline Attitudes Toward Physician's Shared Role & $17.00(4.73)$ & $13.18(3.87)$ & $14.50(3.54)$ \\
\hline $\begin{array}{l}\text { 4. Physicians have the right to alter patient care plans } \\
\text { developed by the team } \dagger\end{array}$ & $2.76(1.56)$ & $2.09(1.30)$ & $2.00(0.00)$ \\
\hline
\end{tabular}


6. A team's primary purpose is to assist physicians in achieving treatment goals for patients $\dagger$

13. The physician should not always have the final word in decisions made by health care teams

\begin{tabular}{ccc}
$3.72(1.31)$ & $3.00(1.18)$ & $1.50(0.71)$ \\
& & \\
$3.96(1.31)$ & $3.36(1.03)$ & $4.50(0.71)$ \\
& & \\
$3.00(1.63)$ & $2.09(1.97)$ & $3.00(1.41)$ \\
$3.56(0.96)$ & $2.64(0.67)$ & $3.50(0.71)$ \\
$74.80(11.85)$ & $74.10(8.96)$ & $83.00(5.66)$ \\
\hline
\end{tabular}

16. The physician has the ultimate legal responsibility

for decisions made by the team ${ }^{\dagger}$

18. Physicians are natural team leaders $\dagger$

ATHCT Total

Note. $\uparrow$ indicates item is reverse coded. ATHCT $=$ Attitude Toward Health Care Teams. Scores: $0=$ Strongly Disagree, $1=$ Moderately Disagree, $2=$ Somewhat Disagree, $3=$ Somewhat Agree, 4=Moderately Agree, 5=Strongly Agree. Total scores range from 0 to 105.

Interprofessional Collaboration Scale. Total scores indicate moderate interprofessional collaboration ( $M=36.94 ; S D=4.13)$, representing $71.04 \%$ of the possible maximum score. A $t$ test indicated significant differences between groups overall scores, $t(32)=3.28, p=.003$, Hedge's $g=1.23$ (see Figure 18). Health care providers reported significantly higher scores $(M=40.10$; $S D=4.36)$ than clinical psychology students $(M=35.63 ; S D=3.29)$, indicating more positive perspectives and experiences with interprofessional team collaboration. In general, participants' scores were highest for the Accommodation factor (representing $75.15 \%$ of the maximum score), while scores for Communication and Isolation were moderately positive, though lower $(69.25 \%$ and $67.17 \%$ of the maximum score, respectively). Significant differences between groups were found for Accommodation and Isolation (see Table 17). For Accommodation, scores by health care providers were significantly higher than those by clinical psychology graduate students, $t(32)=2.11, p=.043$, Hedge's $g=0.79$. Thus, health care providers feel more accommodated by team members than do clinical psychology graduate students. Overall, participants highly endorsed items for this factor indicating that team members are considerate of other team members when treatment planning, were open to reviewing an individuals' issues, worked together to organize patient care, and were open to implementing new, jointly created team practices. Participants also agreed that there was mutual understanding of the responsibilities of 
the various team members, an item related to the Communication factor. No significant differences were found for Communication, $t(32)=1.31, p=.201$, Hedge's $g=0.49$. For Isolation, because Levene's test was significant, the unequal variance statistic was used, indicating that students had significantly higher scores than health care providers, $t(32)=-3.99, p=.002$, Hedge's $g=1.79$. This indicates that students felt more isolated than health care providers. In particular, students largely agreed that some professions consider their work is of greater importance than others and approximately half felt not all team members would be open to exploring new practices as a team, whereas nearly all of the health care providers disagreed with these Isolation items.

Similar to the ATHCT, there are no group norms for the ICS. However, the scores obtained are similar to, though slightly higher than those found in previous research conducted in interprofessional team environments, particularly for health care providers (Gotlib Conn et al., 2014; Kenaszchuk et al., 2011, 2012). Group differences found for Accommodation and Isolation are similar to past research that indicated that physicians experienced less isolation and greater accommodation from team members than other allied health care professionals (Gotlib Conn et al., 2014; Kenaszchuk et al., 2012). Scores for Communication were also similar to past research (Gotlib Conn et al., 2014; Kenaszchuk et al., 2011, 2012). 


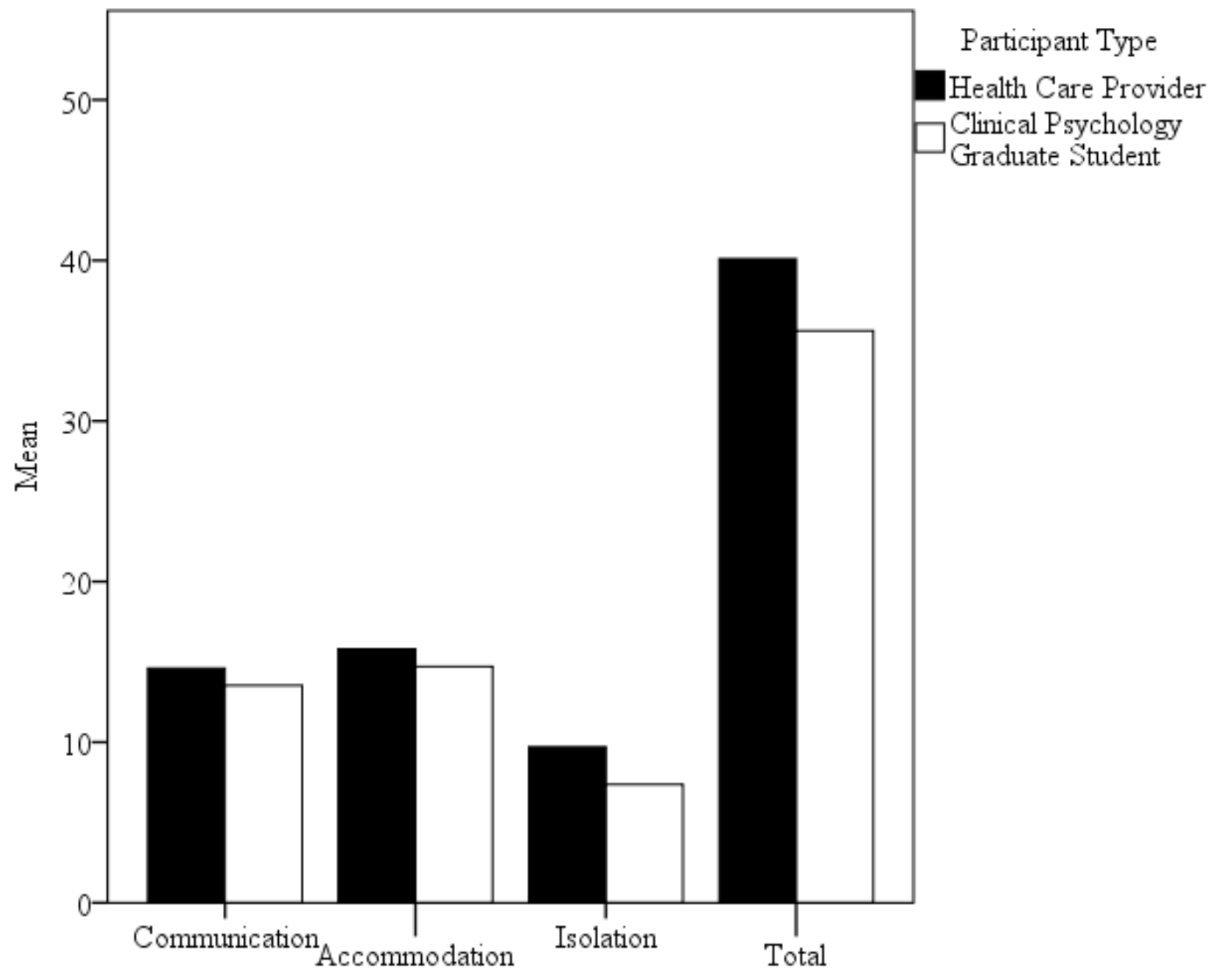

Figure 18. Interprofessional Collaboration Scale total and factor scores. 
Table 17

Comparison of ICS Factors and Scores Between Participant Groups

\begin{tabular}{|c|c|c|c|}
\hline Item & $\begin{array}{c}\text { Graduate } \\
\text { Students } \\
N=24 \\
M(S D)\end{array}$ & $\begin{array}{c}\text { Health Care } \\
\text { Providers } \\
N=10 \\
M(S D)\end{array}$ & $t$ \\
\hline Communication & $13.54(2.19)$ & $14.60(2.07)$ & 0.52 \\
\hline $\begin{array}{l}\text { 1. The team has a good understanding about their respective } \\
\text { responsibilities }\end{array}$ & $2.83(0.64)$ & $3.20(0.42)$ & \\
\hline $\begin{array}{l}\text { 3. I feel that patient treatment and care are not adequately } \\
\text { discussed between and among members } \dagger\end{array}$ & $2.63(0.58)$ & $2.70(0.68)$ & \\
\hline 9. Team members anticipate when they will need others' help & $2.67(0.57)$ & $2.90(0.32)$ & \\
\hline $\begin{array}{l}\text { 10. Important information is always passed between and among } \\
\text { team members }\end{array}$ & $2.54(0.83)$ & $2.60(0.70)$ & \\
\hline 11. Disagreements within the team often remain unresolved $\dagger$ & $2.88(0.68)$ & $3.20(0.63)$ & \\
\hline Accommodation & $14.71(1.37)$ & $15.80(1.40)$ & $2.11 *$ \\
\hline $\begin{array}{l}\text { 2. Team members are usually willing to take into account the } \\
\text { convenience of individuals when planning their work }\end{array}$ & $2.88(0.54)$ & $3.10(0.32)$ & \\
\hline $\begin{array}{l}\text { 4. Individuals on the team share similar ideas about how to treat } \\
\text { patients }\end{array}$ & $2.79(0.42)$ & $2.90(0.32)$ & \\
\hline 5. Team members are willing to discuss individuals' issues & $3.17(0.38)$ & $3.40(0.52)$ & \\
\hline 6. Team members cooperate with the way care is organized & $3.04(0.36)$ & $3.20(0.42)$ & \\
\hline $\begin{array}{l}\text { 7. Team members would be willing to cooperate with new, } \\
\text { agreed upon practices }\end{array}$ & $2.83(0.48)$ & $3.20(0.42)$ & \\
\hline Isolation & $7.38(1.10)$ & $9.70(1.70)$ & $3.99 * *$ \\
\hline 8. Individuals are not usually asked for their opinions $\dagger$ & $2.96(0.62)$ & $3.20(0.42)$ & \\
\hline $\begin{array}{l}\text { 12. Some individuals think their work is more important than } \\
\text { the work of others on the team } \dagger\end{array}$ & $2.00(0.59)$ & $3.20(0.79)$ & \\
\hline $\begin{array}{l}\text { 13. Some individuals would not be willing to discuss new } \\
\text { practices with other team members } \dagger\end{array}$ & $2.42(0.58)$ & $3.30(0.82)$ & \\
\hline Total & $35.63(3.29)$ & $40.10(4.36)$ & $1.24 * *$ \\
\hline
\end{tabular}

Contact with Interprofessional Team Measure: Health care provider version. The

CITM-HCP was administered to health care providers and clinical psychology graduate students.

Significant differences were found between groups on the frequency with which they provide and receive consultation from other health care providers (see Figure 19). Health care providers indicated that on average, they provided a moderate amount of consultation $(M=4.18 ; S D=1.99)$ to other interprofessional team members, whereas students, on average, rarely provided 
consultation $(M=2.38 ; S D=1.53), t(33)=2.95, p=.006$, Hedge's $g=1.07$. It appears that students also did not receive as much consultation, receiving it on average only some of the time ( $M=$ 2.75; $S D=2.31)$, whereas health care providers often received consultation, on average $(M=4.73$; $S D=1.27), t(33)=2.65, p=.012$, Hedge's $g=0.97$ (see tables 18 and 19). However, in each group, the level of interprofessionalism varied, with some not providing or receiving any consultation (particularly in the student group), while others engaged in regular interprofessional consultation, including consulting with at least one other professional in $100 \%$ of their cases.

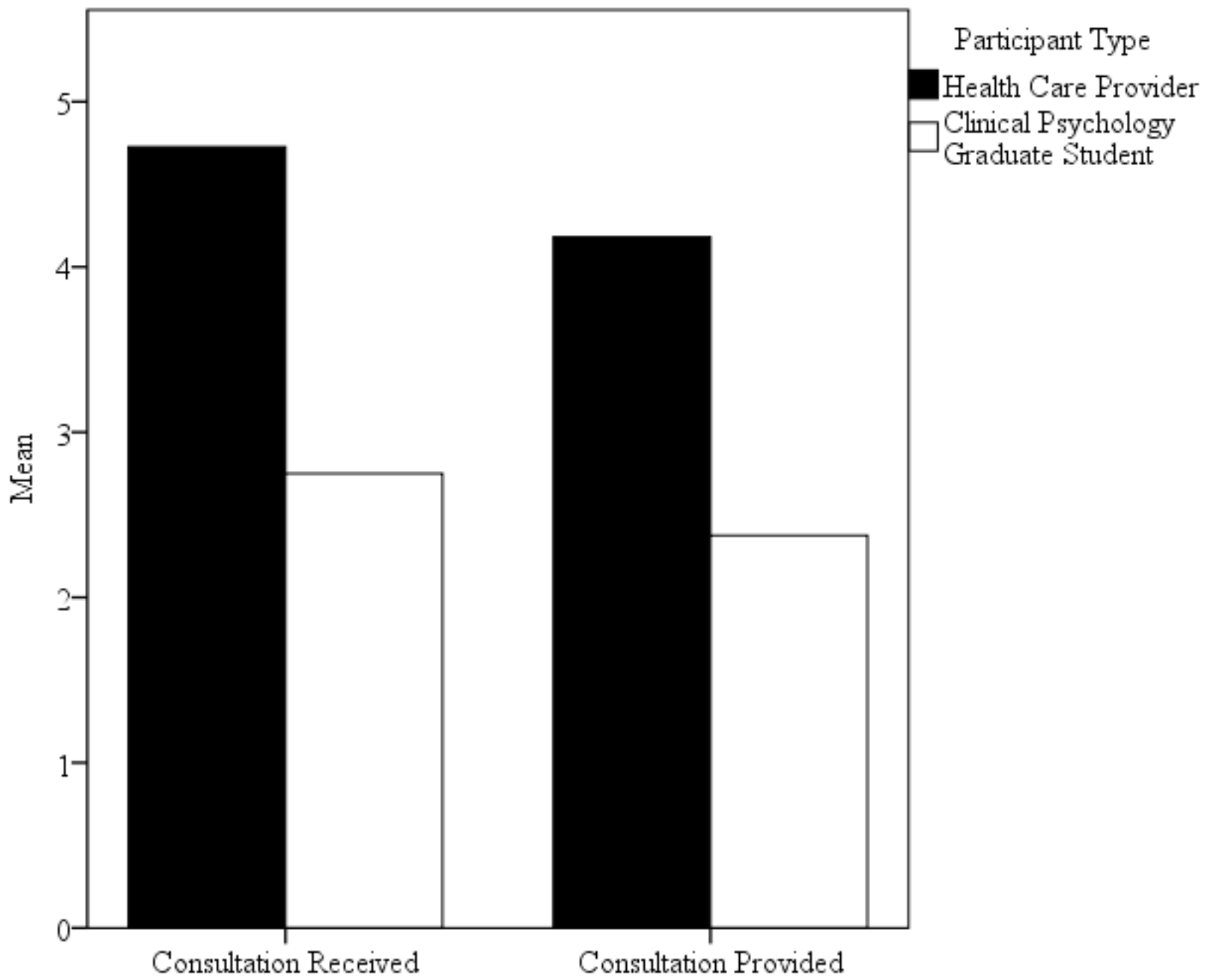

Figure 19. Frequency of consultation provided or received by participants as reported with the Contact with Interprofessional Team Members-Health Care Provider version. 
Table 18

Health Care Provider's Consultation with Other Team Members: Percentage of Patient Cases, CITM-HCP $(\mathrm{N}=12)$

\begin{tabular}{lcccc}
\hline Health Care Provider & \multicolumn{2}{c}{ Provided } & \multicolumn{2}{c}{ Received } \\
\cline { 2 - 5 } & $M(S D)$ & $\begin{array}{c}\text { Max. Value } \\
(\%)\end{array}$ & $\begin{array}{c}\text { Max. Value } \\
(\%)\end{array}$ \\
\hline Administration Staff & $11.27(29.94)$ & 100 & $11.36(15.49)$ & 40 \\
Dental Hygienist/Dentist & $1.18(3.06)$ & 10 & $4.91(7.46)$ & 24 \\
Physician & $33.82(36.16)$ & 100 & $35.09(33.52)$ & 100 \\
Psychiatrist & $0.00(0.00)$ & 0 & $19.45(17.76)$ & 50 \\
Chiropractor & $2.27(5.29)$ & 16 & $23.36(27.09)$ & 100 \\
Social Worker & $0.82(2.40)$ & 8 & $21.36(14.33)$ & 51 \\
Dietitian & $3.27(6.12)$ & 19 & $18.36(12.56)$ & 30 \\
Reception Staff & $3.27(9.05)$ & 30 & $13.91(19.34)$ & 50 \\
Nurse & $32.00(30.33)$ & 91 & $42.82(33.63)$ & 91 \\
Nurse Practitioner & $16.55(16.80)$ & 50 & $17.64(12.24)$ & 32 \\
Pharmacist & $4.00(5.88)$ & 16 & $27.09(19.91)$ & 66 \\
Psychologist & $1.64(3.64)$ & 9 & $16.09(15.07)$ & 48 \\
Other (e.g. Physiotherapist, & $0.91(3.02)$ & 10 & $0.82(2.71)$ & 9 \\
Addictions Counsellor) & & & & \\
\hline Note. ClTM-HCP=Con & & & & \\
\hline
\end{tabular}

Note. CITM-HCP $=$ Contact with Interprofessional Team Members-Health Care Provider version. Percentage of cases ranged from 0 to 100. All categories for health care providers had a minimum value of 0 . Health care providers in this sample included: physicians, chiropractors, and social workers. 
Table 19

Clinical Psychology Graduate Students' Consultation with Other Team Members: Percentage of Patient Cases, CITM-HCP $(\mathrm{N}=25)$

\begin{tabular}{lcccc}
\hline Health Care Provider & \multicolumn{2}{c}{ Provided } & \multicolumn{2}{c}{ Received } \\
\cline { 2 - 5 } & $M(S D)$ & $\begin{array}{c}\text { Max. Value } \\
(\%)\end{array}$ & $M(S D)$ & $\begin{array}{c}\text { Max. Value } \\
(\%)\end{array}$ \\
\hline Administration Staff & $6.64(22.04)$ & 100 & $11.36(15.49)$ & 40 \\
Dental Hygienist/Dentist & $0.00(0.00)$ & 0 & $4.91(7.46)$ & 24 \\
Physician & $11.24(19.59)$ & 81 & $35.09(33.52)$ & 100 \\
Psychiatrist & $5.96(15.74)$ & 60 & $19.45(17.76)$ & 50 \\
Chiropractor & $0.00(0.00)$ & 0 & $23.36(27.09)$ & 100 \\
Social Worker & $8.96(12.42)$ & 41 & $21.36(14.33)$ & 51 \\
Dietitian & $0.80(2.78)$ & 11 & $18.36(12.56)$ & 30 \\
Reception Staff & $5.04(20.21)$ & 100 & $13.91(19.34)$ & 50 \\
Nurse & $0.00(0.00)$ & 0 & $42.82(33.63)$ & 91 \\
Nurse Practitioner & $0.00(0.00)$ & 0 & $17.64(12.24)$ & 32 \\
Pharmacist & $0.00(0.00)$ & 0 & $27.09(19.91)$ & 66 \\
Psychologist & $12.48(26.92)$ & 93 & $16.09(15.07)$ & 48 \\
Other (e.g. Physiotherapist, & $0.00(0.00)$ & 0 & $0.82(2.71)$ & 9 \\
Addictions Counsellor) & & & & \\
\hline Note. CITM-HCP=Cont & & & & \\
\hline
\end{tabular}

Note. CITM-HCP $=$ Contact with Interprofessional Team Members-Health Care Provider version. Percentage of cases ranged from 0 to 100. All categories for health care providers had a minimum value of 0 .

Clinical psychology graduate students. In general, clinical psychology graduate students received more consultation than they provided. Student participants identified receiving varying levels of consultation to all professionals, except nurses, though eight participants indicated receiving no consultation from any team member. Slightly more than half of students indicated consulting with a social worker, while slightly less than half consulted with a physician for at least one case (see Table 20). The next most consulted professionals were psychologists, followed by administrative staff, and psychiatrists. Few students reported receiving consultation from nurses, dental hygienists/dentists, and chiropractic. Contact with other team members for the purpose of providing consultation was lower than consultation received. Student participants indicated providing no consultation to dentists/dental hygienists, chiropractors, nurses, nurse practitioners, or pharmacists and 11 noted providing no consultation to any team member. 
Again, clinical psychology graduate students reported providing the most consultation to physicians, followed by social workers, psychologists, and psychiatrists.

Health care providers. Overall, health care providers reported that they commonly engaged in consultation, though there was some variation among participants. They reported receiving more consultation than they provided to others. The most consultation received by health care providers for at least one case was from pharmacists, followed by physicians, chiropractors, social workers, and nurse practitioners. Fewer received consultation from reception or administrative staff. Health care providers provided the most consultation to physicians, nurses, and nurse practitioners, and least consultation to dental hygienists/dentists, chiropractors, social workers, reception staff, and psychologists, as well as psychiatrists to whom no one indicated providing consultation. Though the small sample makes it difficult to draw conclusions, it should be noted that the two non-physician health care providers indicated higher rates of providing $(M=7.00 ; S D=0.00)$ and receiving consultation $(M=6.50 ; S D=2.12)$ than the nine physicians provided $(M=3.56 ; S D=1.60)$ or received $(M=4.33 ; S D=0.71)$. 
Table 20

Number of Clinical Psychology Graduate Students and Health Care Providers Who Received and Provided Consultation to Another Health Care Provider at the FHT within the Last Year

\begin{tabular}{lcccc}
\hline & \multicolumn{2}{c}{ Graduate Students $(N=25)$} & \multicolumn{2}{c}{ Health Care Providers $(N=11)$} \\
\cline { 2 - 5 } & $\begin{array}{c}\text { Consultation } \\
\text { Received }\end{array}$ & $\begin{array}{c}\text { Consultation } \\
\text { Provided } \\
n(\%)\end{array}$ & $\begin{array}{c}\text { Consultation } \\
\text { Received } \\
n(\%)\end{array}$ & $\begin{array}{c}\text { Consultation } \\
\text { Provided } \\
n(\%)\end{array}$ \\
\hline Administration Staff & $n(\%)$ & $3(12 \%)$ & $6(55 \%)$ & $3(27 \%)$ \\
Dental Hygienist/Dentist & $10(40 \%)$ & $0(0 \%)$ & $5(45 \%)$ & $2(18 \%)$ \\
Physician & $1(4 \%)$ & $11(44 \%)$ & $9(82 \%)$ & $8(73 \%)$ \\
Psychiatrist & $12(48 \%)$ & $5(20 \%)$ & $8(73 \%)$ & $0(0 \%)$ \\
Chiropractor & $8(32 \%)$ & $0(0 \%)$ & $9(82 \%)$ & $2(18 \%)$ \\
Social Worker & $1(4 \%)$ & $8(32 \%)$ & $9(82 \%)$ & $2(18 \%)$ \\
Dietitian & $14(56 \%)$ & $0(0 \%)$ & $8(73 \%)$ & $4(36 \%)$ \\
Reception Staff & $4(16 \%)$ & $3(12 \%)$ & $6(55 \%)$ & $2(18 \%)$ \\
Nurse & $4(16 \%)$ & $0(0 \%)$ & $8(73 \%)$ & $7(64 \%)$ \\
Nurse Practitioner & $0(0 \%)$ & $0(0 \%)$ & $9(82 \%)$ & $8(73 \%)$ \\
Pharmacist & $2(8 \%)$ & $0(0 \%)$ & $10(91 \%)$ & $4(36 \%)$ \\
Psychologist & $2(8 \%)$ & $5(20 \%)$ & $8(73 \%)$ & $2(18 \%)$ \\
\hline
\end{tabular}

Note. FHT=Family health team. Consultation provided or received for at least one patient.

Contact with Interprofessional Team Measure: Patient version. Patient participants

reported that overall, three to four health care providers were involved in their care at the St.

Michael's Hospital FHTs. They indicated that they either occasionally or often received care from more than one health care provider, which included physicians, Social Work, Psychology, and Nursing.

Interdisciplinary Education Perception Scale. No significant differences between clinical psychology graduate students and health care providers were found. The average total score for the IEPS was high $(M=90.94 ; S D=7.90$; Item Mean= 5.05), with health care providers having slightly higher scores $(M=93.70 ; S D=7.68)$ than clinical psychology graduate students $(M=89.68 ; S D=7.85)$, though this was not statistically significant, $t(30)=1.35, p=.187$, Hedge's $g=0.52$. Thus, both groups held positive perspectives on interdisciplinary team collaboration, with the overall average score representing $84.20 \%$ of the potential maximum score (see Table 21). 
Compared to past research, scores obtained in this study are similar to, though somewhat higher than past research, depending on the study (Goelen et al., 2006; Hawk et al., 2002; Lehrer et al., 2015; Luecht et al., 1990; Tsang, Cheung, \& Sakakibara, 2016). Scores were more similar to the baseline scores for at least one study, though the authors of this study noted that baseline scores were higher than expected (Pinto et al., 2012). Results from this study followed a similar pattern as past research with lower scores for Perceived Actual Cooperation $(M=25.81 ; S D=$ 2.68; Item Mean = 5.16). This may indicate that despite generally seeing the need for interprofessional cooperation, this expectation is not be matched by participants' lived experience. Scores for Competence and Autonomy received high scores $(M=41.47 ; S D=4.07)$ that were generally higher than past research. Within this factor, individuals highly endorsed that individuals in their discipline were well-trained and confident in their aims and goals. In contrast, other factor items were rated lower, indicating not being thought of as well by other professionals or experiencing less respect from other team members. 
Table 21

Comparison of IEPS Scores Between Participant Groups (N=32)

\begin{tabular}{|c|c|c|}
\hline Item & $\begin{array}{c}\text { Graduate } \\
\text { Students } \\
N=22 \\
M(S D)\end{array}$ & $\begin{array}{c}\text { Health Care } \\
\text { Providers } \\
N=10 \\
M(S D)\end{array}$ \\
\hline Competence and Autonomy & $40.59(4.18)$ & $43.40(3.20)$ \\
\hline 1. Individuals in my profession are well-trained & $5.45(0.74)$ & $5.90(0.32)$ \\
\hline $\begin{array}{l}\text { 3. Individuals in my profession demonstrate a great deal of } \\
\text { autonomy }\end{array}$ & $5.36(0 . .73)$ & $5.40(0.97)$ \\
\hline $\begin{array}{l}\text { 4. Individuals in other professions respect the work done by } \\
\text { my profession }\end{array}$ & $4.36(1.22)$ & $5.30(0.48)$ \\
\hline $\begin{array}{l}\text { 5. Individuals in my profession are very positive about their } \\
\text { goals and objectives }\end{array}$ & $5.32(0.48)$ & $5.60(0.52)$ \\
\hline $\begin{array}{l}\text { 7. Individuals in my profession are very positive about their } \\
\text { contributions and accomplishments }\end{array}$ & $5.36(0.90)$ & $5.40(0.52)$ \\
\hline $\begin{array}{l}\text { 9. Individuals in other professions think highly of my } \\
\text { profession }\end{array}$ & $4.36(1.09)$ & $5.30(0.48)$ \\
\hline $\begin{array}{l}\text { 10. Individuals in my profession trust each other's professional } \\
\text { judgment }\end{array}$ & $5.14(0.64)$ & $5.10(0.88)$ \\
\hline 13. Individuals in my profession are extremely competent & $5.23(0.69)$ & $5.40(0.52)$ \\
\hline Perceived Need for Cooperation & $10.36(1.50)$ & $10.70(1.57)$ \\
\hline $\begin{array}{l}\text { 6. Individuals in my profession need to cooperate with other } \\
\text { professions }\end{array}$ & $5.68(0.48)$ & $5.70(0.48)$ \\
\hline $\begin{array}{l}\text { 8. Individuals in my profession must depend upon the work of } \\
\text { people in other professions }\end{array}$ & $4.68(1.25)$ & $5.00(1.49)$ \\
\hline Perception of Actual Cooperation & $25.86(2.78)$ & $25.70(2.58)$ \\
\hline $\begin{array}{l}\text { 2. Individuals in my profession are able to work closely with } \\
\text { individuals in other professions }\end{array}$ & $5.36(0.73)$ & $5.20(0.42)$ \\
\hline $\begin{array}{l}\text { 14. Individuals in my profession are willing to share } \\
\text { information and resources with other professionals }\end{array}$ & $5.41(0.73)$ & $5.30(0.95)$ \\
\hline $\begin{array}{l}\text { 15. Individuals in my profession have good relations with } \\
\text { people in other professions }\end{array}$ & $5.14(0.64)$ & $5.20(0.42)$ \\
\hline $\begin{array}{l}\text { 16. Individuals in my profession think highly of other related } \\
\text { professions }\end{array}$ & $4.73(0.88)$ & $4.90(1.10)$ \\
\hline 17. Individuals in my profession work well with each other & $5.23(0.69)$ & $5.10(0.88)$ \\
\hline Understanding Others' Value & $12.86(2.66)$ & $13.90(2.42)$ \\
\hline $\begin{array}{l}\text { 11. Individuals in my profession have a higher status than } \\
\text { individuals in other professions }\end{array}$ & $3.45(1.57)$ & $3.70(1.64)$ \\
\hline $\begin{array}{l}\text { 12. Individuals in my profession make every effort to } \\
\text { understand the capabilities and contributions of other } \\
\text { professions }\end{array}$ & $4.68(1.25)$ & $4.50(1.08)$ \\
\hline $\begin{array}{l}\text { 18. Individuals in other professions often seek the advice of } \\
\text { people in my profession }\end{array}$ & $4.73(1.03)$ & $5.70(0.48)$ \\
\hline Total & $89.68(7.85)$ & $93.70(7.68)$ \\
\hline
\end{tabular}


Note. IEPS $=$ Interdisciplinary Education Perception Scale. Scores: $1=$ Strongly Disagree, $2=$ Moderately Disagree, $3=$ Somewhat Disagree, $4=$ Moderately Agree, and 5= Strongly Agree. Total scores range from 18 to 108 .

\section{Clinical Psychology Graduate Student Training}

\section{Self-Efficacy for Interprofessional Experiential Learning-Revised.}

SEIEL-R factor analysis. Because this measure was altered from its initial 16-item two factor version to 12 items, a principal axis factor analysis with Varimax rotation was conducted. Two factors were imputed based on the a priori two factor model, which was confirmed by the scree plot (see Table 22). This method was deemed more appropriate rather than Kaiser's criterion, which indicated three factor, is more likely to overestimate the number of factors which is particularly problematic in small sample sizes (De Winter, Dodou \& Wieringa, 2009; Field, 2012; Zwick \& Velicer, 1986). The Kaiser-Meyer-Olkin analysis indicated sampling adequacy,

$K M O=.67$, and Bartlett's Test of Sphericity was significant, $\chi^{2}(66)=205.52, p=.000$. Factor 1 accounts for $35.80 \%$ of the variance and appears to be highly reliable $(\alpha=.90$.). Factor 2 is similarly reliable $(\alpha=.88)$ and accounts for $33.47 \%$ of the variance. Reliability for the SEIEL-R overall was high $(\alpha=.93)$.

The two factors extracted were similar to, but did not replicate the two factors found by Mann et al. (2012). This is unsurprising given that items were omitted and wording changed to better reflect the training in a psychology training clinic rather than interdisciplinary classroom learning. The first factor was labelled Interprofessional Understanding and Capabilities and the second factor labelled Interprofessional Team Goals and Feedback. There is some evidence to suggest that because only two factors were extracted and several high factor loadings were found, there may be veracity to this factor structure (De Winter et al., 2009; Fields, 2012; Yong \& Pearce, 2013; Zwick \& Velicer, 1986). Nonetheless, further research of this revised version 
with a greater sample size is needed to determine its reliability (Yong \& Pearce, 2013; Zwick \&

Velicer, 1986).

Table 22

Factor Loadings for the Self-efficacy for Interprofessional Experiential Learning-Revised with Varimax Rotation ( $\mathrm{N}=21)$

\begin{tabular}{|c|c|c|c|}
\hline Scale Item & $\begin{array}{l}\text { Understanding } \\
\text { and } \\
\text { Capabilities }\end{array}$ & $\begin{array}{l}\text { Team Goals } \\
\text { and } \\
\text { Feedback }\end{array}$ & Communalities \\
\hline $\begin{array}{l}\text { 1. Working with different professionals to form } \\
\text { a team. }\end{array}$ & .72 & & .68 \\
\hline $\begin{array}{l}\text { 2. Working with different professions to } \\
\text { resolve problems in the team. }\end{array}$ & .72 & & 67 \\
\hline $\begin{array}{l}\text { 3. Working with different professionals to } \\
\text { develop a realistic appropriate patient care plan. }\end{array}$ & & 63 & .61 \\
\hline $\begin{array}{l}\text { 4. Working with different professionals to } \\
\text { understand our respective roles in an } \\
\text { interprofessional team. }\end{array}$ & .80 & & .76 \\
\hline $\begin{array}{l}\text { 5. Working with different professionals to } \\
\text { understand the benefits to patients of team care. }\end{array}$ & .74 & & .62 \\
\hline $\begin{array}{l}\text { 6. Understanding and discussing the objectives } \\
\text { of interprofessional learning. }\end{array}$ & .88 & & .78 \\
\hline $\begin{array}{l}\text { 7. Interacting with professionals and disciplines } \\
\text { different from my own. }\end{array}$ & & .76 & .70 \\
\hline $\begin{array}{l}\text { 8. Providing feedback to individual } \\
\text { interprofessional team members on their } \\
\text { function and work on the team. }\end{array}$ & & .91 & .83 \\
\hline $\begin{array}{l}\text { 9. Helping the patient to understand the } \\
\text { objectives of the interprofessional learning. }\end{array}$ & & .54 & .54 \\
\hline $\begin{array}{l}\text { 10. Evaluating the quality of the work as an } \\
\text { interprofessional team. }\end{array}$ & 64 & & .73 \\
\hline $\begin{array}{l}\text { 11. Evaluating the degree to which an } \\
\text { interprofessional team has achieved its goals. }\end{array}$ & & .76 & .71 \\
\hline $\begin{array}{l}\text { 12. Communicating effectively with other } \\
\text { members of an interprofessional team. }\end{array}$ & & .73 & .69 \\
\hline
\end{tabular}

SEIEL-R scores. Given that the revised scale includes four fewer items than the original version, average item scores are also presented for a point of comparison with previous research using the SEIEL. Overall, clinical psychology graduate students reported moderate to high confidence in their self-efficacy and ability to work in an interprofessional team and learning 
environment with an average score of $88.62(\mathrm{SD}=13.50$; Item Mean=7.39) and a range of 64 to 108 (see Table 23 for more detail). Scores of 7 or higher are considered to be high, though also average for individuals with previous interprofessional experiences (Oza et al., 2015; Vari et al., 2013). The average for the Interprofessional Understanding and Capabilities factor was 44.90 $(S D=6.91 ;$ Item Mean= 7.29 $)$ and the Interprofessional Team Goals and Feedback was 43.71 $(S D=7.60$; Item Mean=7.48). Of the twenty-five clinical psychology graduate students, only 21 completed this measure. Five of those completed the measure while completing the PTC practicum and the remaining 16 were past PTC practicum students, who have likely received greater clinical training at the point of data collection. When comparing both groups, past students $(M=92.50 ; S D=12.08)$ had significantly higher scores than current students $(M=76.20$; $S D=10.55), t(19)=2.70, p=.014$, Hedge's $g=1.38$. These scores are similar, though slightly lower than those found in past research, with some variability between studies (Mann et al., 2012; Oza et al., 2015; Vari et al., 2013).

The highest rated items, those with a score of 8 or higher, represent participants' confidence in their ability to work with health care providers from different fields, understand the varying roles of team members, engage with team members from different backgrounds, and the ability to effectively communicate with team members. Alternatively, the lowest rated items represent less confidence in giving team members specific feedback on their work, conducting an evaluation of the quality of the teams' interprofessional performance, and whether or not the teams is meeting its intended goals. 
Table 23

Self-Efficacy for Interprofessional Experiential Learning-Revised Scores for Clinical Psychology Graduate Students $(\mathrm{N}=21)$

\begin{tabular}{lcc}
\hline Scale Item & $M(S D)$ & $\begin{array}{c}\text { Score } \\
\text { Range }\end{array}$ \\
\hline $\begin{array}{l}\text { Understanding and Capabilities } \\
\text { 1. Working with different professionals to form a team. }\end{array}$ & $\begin{array}{c}44.90(6.91) \\
7.67(1.77)\end{array}$ & $\begin{array}{c}29-57 \\
2-10\end{array}$ \\
$\begin{array}{l}\text { 2. Working with different professions to resolve } \\
\text { problems in the team. }\end{array}$ & $7.43(1.50)$ & $4-10$ \\
$\begin{array}{l}\text { 4. Working with different professionals to understand } \\
\text { our respective roles in an interprofessional team. }\end{array}$ & $8.10(1.22)$ & $6-10$ \\
$\quad \begin{array}{l}\text { 5. Working with different professionals to understand } \\
\text { the benefits to patients of team care. }\end{array}$ & $7.95(1.12)$ & $6-10$ \\
$\begin{array}{l}\text { 6. Understanding and discussing the objectives of } \\
\text { interprofessional learning. }\end{array}$ & $7.52(1.23)$ & $5-10$ \\
$\begin{array}{l}\text { 10. Evaluating the quality of the work as an } \\
\text { interprofessional team. }\end{array}$ & $6.24(1.48)$ & $3-8$ \\
Team Goals and Feedback & $43.71(7.60)$ & $27-56$ \\
$\begin{array}{l}\text { 3. Working with different professionals to develop a } \\
\text { realistic appropriate patient care plan. }\end{array}$ & $7.86(1.53)$ & $5-10$ \\
$\begin{array}{l}\text { 7. Interacting with professionals and disciplines } \\
\text { different from my own. }\end{array}$ & $8.14(1.28)$ & $6-10$ \\
$\begin{array}{l}\text { 8. Providing feedback to individual interprofessional } \\
\text { team members on their function and work on the team. }\end{array}$ & $5.95(1.96)$ & $2-9$ \\
$\begin{array}{l}\text { 9. Helping the patient to understand the objectives of the } \\
\text { interprofessional learning. }\end{array}$ & $7.00(1.70)$ & $4-10$ \\
$\begin{array}{l}\text { 11. Evaluating the degree to which an interprofessional } \\
\text { team has achieved its goals. }\end{array}$ & $6.76(1.58)$ & $3-9$ \\
$\begin{array}{l}\text { 12. Communicating effectively with other members of } \\
\text { an interprofessional team. }\end{array}$ & $8.00(1.45)$ & $4-10$ \\
Total
\end{tabular}

Note. Scores range from 1 to 10 , where $1=$ Low Confidence and 10= High Confidence. Total scores can range from 12 to 120 .

\section{Correlations Between Measures}

Significant correlations were found between the IEPS and ATHCT $(r=0.36, p=.042)$ and ICP ( $r=0.47, p=.006)$. Thus, there appears to be convergent validity among the measures.

However, a significant correlation was not found between the ATHCT and ICP $(r=0.19, p=.290)$. This may be related to the small sample size. The SEIEL-R was not significantly correlated with any measure. This may be because it was adapted from its original version and further 
examination of the revised measure is warranted. The CITM was informative in nature and does not include a total score. As such, correlations with this measure were not calculated.

\section{Phase 2 Interviews: Methods}

\section{Participants}

Seventeen participants consented to individual interviews, including nine clinical psychology graduate students, four health care providers, three clinical supervisors at the PTC, and one patient. Student participants represented a range of years since the PTC has been open. The four health care provider participants represented four different disciplines. Further identifying information was purposely not collected to protect participants' confidentiality.

\section{Materials}

Interview questions. Research literature on intervention implementation (Cane, O’Connor, \& Michie, 2012; Michie et al., 2005), interdisciplinary FHT functioning (e.g. Goldman et al., 2010; Mulvale \& Bourgeault, 2007; Mulvale et al., 2008), and clinical psychology graduate training programs (e.g. Schulte et al., 2004; Talen et al., 2005) were consulted in the development of contextual factors and interview questions (see Appendix S). Questions were based on three key domains (patient care, interprofessionalism, and the nature of receiving care from a training clinic) and six contextual factors (knowledge, environmental context and resources, team vision and scope of practice, communication, satisfaction, and competency and confidence). For example, to examine patient care focusing on environmental contexts and resources, participants were asked, "To what extent do physical or resource elements (e.g., location of the PTC at the Health Centre at 80 Bond, transportation difficulties) facilitate patient care with regards to services and access to services provided at the PTC?" To investigate interprofessional team functioning and team vision, participants were asked, "When 
thinking about the St. Michael's Hospital FHTs, including the PTC, what are your impressions on whether there is hierarchy of health care professionals versus a team-based, collaborative approach?" And lastly, to explore perspectives on the PTC as a training clinic with regards to knowledge, participants were asked, "Do you feel that graduate students have a good understanding of interdisciplinary functioning and professional roles at the St. Michael's Hospital FHTs?"

Nvivo. Transcripts were coded using Nvivo, an analysis software for qualitative research. It was used to conduct a thematic analysis and identify participant quotations.

\section{Procedure}

Recruitment. Recruitment took place between March and July 2015. Four participant groups were recruited for Phase 2: 1) past or present patients of the PTC; 2) health care providers at St. Michael's Hospital FHTs, including physicians, nurses, nurse practitioners, and allied health practitioners (e.g. social worker, dietitian, chiropractor, pharmacist); 3) past or present Ryerson University clinical psychology graduate students who trained at the PTC; and 4) clinical supervisors at the PTC. Patients were referred to the study by health care providers and graduate students at the PTC, as well as flyers in the FHT waiting rooms. Health care providers, clinical supervisors, and clinical psychology graduate students were recruited through St. Michael's Hospital or Ryerson University internal email by administrative staff not associated with the study or research assistant not associated with the PTC. Flyers were also placed in staff mailrooms and seating areas (e.g. computer room, lunch room). Materials used to recruit participants included an overview of the study, compensation (i.e. light refreshments), and the researchers' contact information. Lastly, some participants were recruited from the online questionnaire in Phase 1. Those who indicated their interest in participating in an interview were 
contacted by a research assistant and a mutually available time was arranged for the interview to take place.

Interviews. Interviews were conducted at Ryerson University, the Health Centre at 80 Bond, or at a health care providers' office by a trained researcher. These were first proposed as focus groups. However, due to scheduling difficulties with participants and requests for one-onone interviews rather than focus group participation, individual interviews were conducted. This provided greater confidentiality and privacy of participants' information.

Prior to starting the interview, participants were provided a written informed consent (see Appendix T), which was reviewed verbally by the research assistant. Questions about the informed consent process and the study in general were encouraged. Light refreshments (e.g. water, juice, granola bars) were also provided. The researcher then asked participants the interview questions relating to patient care, interprofessionalism, and student training at the PTC. At the end of interview, patients were given a written debriefing, as well as compensation for their time (i.e. $\$ 5$ Tim Hortons gift card) and travel expense (two TTC tokens). Interviews ranged in duration from fifteen to fifty-five minutes in length, though the majority took approximately 30 minutes to complete. Each interview was audio-recorded and later transcribed.

Pragmatic lens and thematic analysis. Due to realities of conducting research in a real world context, a pragmatic lens was used to examine participants' responses regarding their experiences at the PTC and St. Michael's Hospital FHT. A pragmatic lens is a theoretical lens by which people can gain and interact with knowledge. Pragmatism posits that knowledge is what is learned as a result of behaviours, consequences, or events and treating information more or less at face value or as intended (Creswell, 2009; Patton, 2002). In this manner, pragmatics allows for flexibility and applicability, and acknowledges that perspectives are subject to natural 
changes over time (Creswell, 2009; Dures et al., 2011). Moreover, this approach extols the benefits of using mixed-methodology to uncover a more full understanding of phenomena, which can lead to more practical or functional usages (Creswell, 2009; Durees et al., 2011; Maxcy, 2003).

Data was analyzed using a thematic analysis. A thematic analysis allows for the identification of themes and patterns in the data in a way that is flexible and retains detail (Boyatzis, 1998; Braun \& Clarke, 2006). Pre-determined themes were derived from the same research literature consulted for the development of interview framework and questions (e.g. communication, satisfaction, and respect among team members). Additional themes were inductively generated during the coding process based on participants' responses (Boyatzis, 1998; Mulvale et al., 2008).

To start, the principal researcher and a research assistant coded the first three transcripts independently in Microsoft Word to achieve consistency among themes and content (Mulvale et al., 2008). The two researchers met to refine the codes and create an initial codebook (Boyatzis, 1998). Researchers then coded four additional transcripts independently and met again and confirmed that the two researchers applied the themes reliably. Through this iterative process, some codes were refined and the codebook was revised. One transcript was then re-coded independently by both raters using the finalized codebook in Nvivo, yielding an agreement average of $97.78 \%$. The principal researcher then recoded all transcripts and codes were iteratively refined.

\section{Phase 2 Interviews: Results}

A final 28 codes were generated, grouped by seven overarching categories. For an overview, see Table 24. Saturation appears to have been met, with no new codes being 
generated near with the addition of later transcripts. Codes occurred frequently among

transcripts, being present in eight or more of the seventeen transcripts.

Table 24

Summary of Interview Themes

Divergent Perspectives Resulting from an Emerging Interprofessional Treatment and Training Model

Mixed feedback

Tension resulting from competing perspectives and demands

Inherent challenges of a new model/clinic

Competing FHT and PTC goals for students

Improvements and changes over time

Physical Features and Collocation of the SMH FHT/PTC

Location and physical features

Collocation of services

Addition of the PTC to the SMH FHTs

Positive addition/essential service

Complements other services

High quality patient care at the PTC

Long wait times

Perspectives on Interprofessionalism and Teamwork

Culture of respect, openness, and equality

Limited hierarchy experienced

Scope of practice

Case by case interprofessional teamwork

Limited direct interprofessional communication

Level of interprofessionalism is person/discipline-driven

Lack of time

Proximity

Benefits of Student Training at the PTC

Positive learning experience overall

Integration of evidence-based learning

Clinical leadership

Resources

Experiential learning about FHTs and interprofessionalism

Drawbacks with Student Training at the PTC

Lack of specific FHT/interprofessional training

Limitations of training opportunities provided at the PTC

Clinical Supervision

Mixed quality of individual supervision

Different supervision modalities 


\section{Overarching Themes}

It is important to first identify a number of themes that overlay the codes that follow on patient care, interprofessionalism, and student training. First, a common theme when looking at the data as a whole was that there were divergent perspectives among individuals, even those from the same participant group, though group differences occurred as well. Participants also identified tensions related to competing demands, differing expectations, and the general difficulties of opening a new St. Michael's Hospital FHT location (i.e. Health Centre at 80 Bond at the time) and the PTC housed within it. As well, participants explained that a number of initial challenges were addressed or were improving with time. Since these varying perspectives were noted across themes, unique codes were created to fully capture the information provided by participants.

\section{Divergent perspectives resulting from an emerging interprofessional treatment and}

\section{training model.}

Mixed feedback. Across participants, patterns of mixed feedback were identified. This highlights differences among individuals and between participant groups. Although there were some areas of agreement sufficient to form consistent codes, participants also voiced divergent feedback, resulting in non-unified or mixed themes, as well as some outliers. For example, participants described mixed experiences with the team-based approach, levels of interprofessional interaction, and quality of student supervision. As well, the majority reported a culture of respect among the team; however, some noted instances where there a lack of respect was experienced.

\section{Team-based functioning.}

Team functioning? I'm not sure that there really is like an established team I guess at St. Mike's... (Participant 8, Student) 
So, it really did feel like it was a team-based approach to patient care. (Participant 1 , Student)

\section{Interprofessional communication.}

From time to time, I kind of go down and also just chat with the students to get a sense of what they are doing, the work that they doing with my patients. So as much as they send up the formal report, just going downstairs and chatting with the supervisor and the students at the same time. Giving them my sense as to what I saw and then hearing from them their more expert opinion has been great. (Participant 13, Health Care Provider)

I would have thought it would have been on the GPs' side of things to come and talk to me and understand what's going on to, you know, understand what they are getting into. And most of the times it seemed like the reverse. (Participant 2, Student)

\section{Culture of respect.}

So I think St. Mike's is excellent in terms of there being a respectful environment. I have had very few instances where I have noticed that there wasn't respect or I felt that my students weren't being treated well or maybe were being treated in terms of other professionals looking down on them or not respecting them. I think that is has been wonderful. And of course students have been very respectful of the different professions as well. But we have had a couple of instances where messages have been sent or a lack of communication or a misunderstanding. Where the communication failed, there has been a lack of respect. (Participant 14, Clinical Supervisor)

Tension resulting from competing perspectives and demands. Participants described a number of understandable challenges arising from the shift from a traditional single-provider model to interprofessional team-based care and the integration of professionals with different backgrounds and levels of experience. For example, tension and differing expectations were noted regarding room use, time spent with patients, and time documenting patient contact, which varied among professionals. Tension also arose due to continued lack of understanding and respect for the varying scopes of practice and certain professionals. As well, difficulties resulting from balancing the needs of student training, the needs of patients with complex mental disorders, and the needs of the FHT model were noted, including: complex patient population 
versus typically first time student psychotherapists; short-term versus long-term treatment due to

waitlist; and perceptions about maximizing treatment room use at the PTC.

\section{Room usage.}

I think there is some space downstairs that I wouldn't say is unused, because it is being used by clinical psychology, but it is not $100 \%$ full all the time. It would be great if we could bring in more clinical trainees and use the space during the time when it is empty. (Participant 13, Health Care Provider)

I think it would be lovely to have a little bit more space, I think when we need flexibility in terms of rooms we don't always have it. So on certain days there isn't an extra room. There isn't always a place for people to sit down and be able to type out notes. There has been talk about losing that larger group room. And I think that is a challenge.

(Participant 14, Clinical Supervisor)

\section{Lack of understanding and respect for other professionals.}

We could be utilized better, I believe. That does come down to not knowing and to some extent lack of respect. You know, let's face it, there is sometimes also a social class aspect to this. People who are [in my field] are often not perceived as professionals with a social class lens, if you like. So we are seen as...it was interesting, I asked a doctor not a long time ago, "so, what would you see as comparable for [individuals in my field] outside the health system?" And their reply was an orderly. And that was quite shocking because often we have first degrees and often Master's education on top along with a lot of additional certifications. And we do actually have a very solid grounding, which isn't readily recognized. (Participant 15 , Health Care Provider)

\section{Varying needs of the clinic, patients, and the FHT model.}

I don't think that has anything to do with it, being a student clinic. I think just because the issues are complex, and there's a lot of comorbidity, and a lot of severity, and it really just pretty difficult to accomplish in 12 to 15 sessions. So, on the one hand, you know, it might be nice to have some flexibility to go beyond that. On the other hand, evidencebased treatments usually are pretty short-term. You don't see people for really extended period. And, I think it's good to be able to, to offer some treatment to a larger number of people than to want treatments, but only be able to see, you know, much smaller percentage of patients in a year. (Participant 5, Clinical Supervisor)

I don't know about changing scopes of practice, if anything it is just about clearly defining what the scope is in a way that is clear so that other professionals know when and what to refer to us and what they can expect of us and what they can't expect of us. And especially, again because we are a training clinic, there are certain things that we won't really treat because we just don't have enough experience and we are still learning. So I think just making it clear that this is what we can offer and this is what we can't 
offer, and this is what we do here and these are the types of things that you should refer to us. (Participant 12, Student)

Inherent challenges of a new model/clinic. Participants outlined a range of challenges inherent to implementing a new model and opening a new clinic (i.e. new FHT location, the PTC), one that is still in the process of finding its way to achieve its mandate and cement its identity. Elements, such as the mental health treatment referral process, scopes of practice, personnel changes, and scheduling problems, were each noted as evolving over time to address initial challenges and new needs that arose. As well, some participants, particularly students, described a lack of clear organization, guidelines, and structure in relation to the PTC, but also interprofessionalism within the St. Michael's Hospital FHTs.

Because obviously interprofessional work is a work in process and family health team model is still fairly new, so we are all still learning about each other's roles and how we can collaborate, what our scope of practice is, and so on. Contributions vary. Sometimes they are also a little porous. So the best person is the person that is available and has the right skills to deal with the issue at the time. So it doesn't necessarily have to be a doctor specifically for a particular issue and different professionals start and then join together. (Participant 15, Health Care Provider)

The second, relatedly actually, challenge, was that, we were often getting referrals for thing that, and for challenges that we could not help people with. So things that were beyond the scope of our practice. Specially, considering most the clinicians there, are first year, so their first practicum. It's not ideal if you're treating patients with schizophrenia, or psychosis, or something along those lines. And then we developed a system to, kind of weed those out, and traffic them towards services that actually help them. And, I think that system been working. (Participant 6, Student)

At that time I think we were also just figuring out like the system, what the setup would be, how the clinic would just run on a daily basis. That was still being sorted out. So, there wasn't really much of a system. We were kind of making things up as we went along it seemed like. (Participant 7, Student)

But yeah, there was a lot of disorganization and I am sure [name of clinical supervisor] felt very overwhelmed cause there were so many of us, and constantly asking questions that [they] didn't have the answer to half the time. (Participant 16, Student) 
Competing FHT and PTC goals for students. Participants described competing demands

of the PTC as a training clinic versus a primary health care clinic providing services to a diverse high needs patient population. Participants reported a mismatch between the complex needs of clients and typically first time practicum students who are seeing clients often for the first time. Respondents noted that although the PTC primarily seeks to provide CBT, due to the complex patient population, treatment can end up being more supportive in nature. As a result, student participants voiced a desire for greater training in CBT-specific skills, supportive therapy (e.g. common factors), and other treatment modalities. As well, respondents noted how FHT and interprofessional training was often secondary to foundational clinical training, namely diagnosing, assessing, and psychotherapy.

I guess one of the challenges that I've experienced, and I know other students in my cohort have, are that the clients that we see are very complex in terms of their comorbidities, their psychosocial stressors. They often have a lot of things going on and this is sort of the very beginning of our training. So, I think one of the challenges is just been being able to actually learn specific sort of CBT skills and learn sort of how to conceptualize a client so that we can help them in the best way that we can. That's definitely been a challenge in terms of clients. (Participant 8 , Student)

I think one of the big challenges is just that the patients we get referred here, on average, I would say are pretty severe and there's a lot of complex comorbidity. And I think that's tough in a training clinic, especially for first practicum placements, because, you know, many people are just trying to figure out how to learn a treatment protocol for the first time. And that can be hard enough with somebody who just has one...defined DSM disorder. And it's so rare here to actually come across that in clients who were referred. So, not just learning how to do treatment but how to manage lots of different things, in the context of, you know, comorbid diagnoses. And then also within just short term treatment. So, even with the, the most severe of clients, typically we have 15 session or less... and you can make a dent in some of the stuff in 15 sessions, but it's, I think, really tough for those reasons...And a lot of students feel like they can't do a really, really good job with many clients given some of those particular, sort of, confines. (Participant 5, Student)

And then, ok so I was saying it is developmental. So, you know we hold students, and we help them out at the level where they are at and then you know, we help them with the level of complexity. But that said, I think when you are first learning CBT or you are first learning DBT, or whatever it may be, it can be challenging when your cases don't 
lend themselves to following a manualized type of approach. And so many times we are having to be creative to draw upon different approaches. And that idea, like I always teach my students about the idea of fidelity with flexibility. And the idea that you need to understand treatment principles and be able to follow treatment principles in an evidenced-based manner, but sometimes we won't be able to follow that evidence-based approach. So, I think that it is great learning because to be honest, I think that that is more representative of what real life is like. But, on the flip side, I think that it is a challenge because I think when you are first starting out one of the things that can be most beneficial is to see the entire, like a full CBT complete protocol from start to finish in the way that it should be under ideal circumstances, so that you have that frame. Because once you have the frame it is much easier to be flexible and to be able to think about how do principles fit into that bigger picture. (Participant 14, Clinical Supervisor)

No. I mean I think the clinic has done an amazing job of... it has two purposes, right? It is part of St. Mike's servicing St. Mike's patients and connecting to doctors, but remembering that it is a training clinic and it has to service the needs of the students. That is not an easy tightrope to walk and so I think it has done a really brilliant job of being part of St. Mike's, but also being a training clinic for students and being aware of its function and purpose and staying true to that. And being very aware that it is there to serve patients as well. So, there are so many different layers of who is getting helped here, you know, it is not an easy sort of mission or mandate. (Participant 11, Health Care Provider)

Improvements and changes over time. Participants noted that there were improvements over time to address a range of challenges and developing issues, including quality of supervision, the referral process, interprofessional communication, and student training. With regards to the PTC specifically, respondents stated that overall, staff were open to and implemented feedback; however, some problems continued to persist. In general, participants relayed a hopeful and positive trajectory toward addressing lingering issues.

Yeah, so communicating with my supervisor in terms of deadlines, I think could have been improved. And, interprofessionally, it was just something we were working on, I think, I think there could have been more, but it's getting better. And even from the beginning of my experience to the end of it, I got more comfortable talking to other professionals and, involving them in the care. So I think, it maybe was just increased comfortability and familiarity, that it became a smoother process. But I definitely saw improvement as time went on. (Participant 4, Student)

I think if anything, the care has only improved. So from my understanding, now they're audio and video recording treatment and assessments and providing feedback. There's a much more structured process. I know the [clinical supervisor name] who's a supervisor 
there and [they are] great in terms of more structured feedback and kind of shaping and guiding. And there are other supervisors there who are kind of filling in, I don't know if they're filling or they supervise specific roles, but I think that, all of that has been sorted out, that not only improves the training of the clinicians, but it's resulting in better patient outcomes or what I hope is better patient outcomes. (Participant 9, Student)

And, when the clinic started seeing clients, or, clinicians were just given clients as they came, you would do a lot more therapy within, you know, up to maybe 25 sessions versus 10 to 15 . Waitlist was huge, clinicians were given things they couldn't handle as a first year practicum student. And that all changed... the scope of practice is very limited to thing we should be treating, and we are capable of treating, and things that we know we can treat. So, I think that because of that, the scope of the general practice of Psychology is somewhat limited, but is still helpful. Because we are doing things that we should be doing, we know we can help clients with. And then if we either something else, we send them to someone else who can help them. (Participant 6, Student)

But now, you know, given our referrals system we're trying to streamline that better and work on that to help the students kind of titrate into those kinds of situations. Initially when I came on, just before I started, you know, they were even taking clients who were in an immediate crisis, which is just I think not something that is beneficial, for both the patient and the student. (Participant 10, Clinical Supervisor)

\section{Patient Care}

The following are elements participants described relating to patient care, including accessing care (physical features and location), the impact of the addition of the PTC to St. Michael's Hospital's FHTs, the ways in which the PTC services complement other FHT services, and the high quality of psychological services, as well as the main drawback, the long wait time for services at the PTC.

\section{Physical features and collocation of the St. Michael's Hospital FHTs/PTC}

Location and physical features. Participants described the St. Michael's Hospital FHT

that houses the PTC as being located centrally downtown, close to the main hospital and associated universities, and generally easily accessible by public transit and cyclists (e.g. bike racks available). Some noted transit issues and associated costs for patients who do not live near the PTC or those associated with other St. Michael's Hospital FHT locations, though some 
compensation for patients in need is available. As well, respondents stated that reception staff were responsive to patients' needs by calling taxis or wheelchair accessible transport when required. The physical space was described as pleasant and positive overall (e.g. good rooms, accessible washrooms, and ramps). The one consistent problem denoted was related to the elevator which was inconsistent and in disrepair for a long period of time, though it was reported to be recently fixed.

Well this particular site is very central. It is very close to all of the main universities. It is right by Dundas Square so the TTC is right on the doorstep, the subway system, the TTC. Accessibility on site too, I have mentioned the familiarity with the building which is quite important to some patients. So they've worked out how long it takes to get here for medical appointments, so it will help them be here for psychology treatment cause they already know how to get here. (Participant 15, Health Care Provider)

It's great. It's nearby St. Mike's, nearby subway. The whole building is like brand new, renovated. All the clinic rooms are really nice. (Participant 17, Patient)

I thought we were ideally situated in the city for most people to access services. I was, I had many clients who would often complain about difficulties getting in, but they were coming in from periphery and I would have someone coming in from, you know, almost [neighbouring city], you know, and some were coming from [Toronto suburb] both complaining about the same issue... So, I don't think I would have solved anyone's problems if we, you know were out in [neighbouring city]. I think, so I think in terms of centralization, I think we're excellent. We're very close to Yonge and Dundas square. (Participant 2, Student)

Okay, so you know there are ramps to the washrooms, which is fantastic. And it's right downtown, close to the subways, which I think is great. And I think the, like the admin staff, the front of house people are so good about calling taxis and, you know, being there for wheel-trans, which I think is also really good. And because it's so, the building...is so close to the main St. Michael's Hospital, so that, also again when it comes to, you know, getting everything done in one day, it might just facilitate, you know, having your blood drawn at the same time as you have you CBT appointment. So that's great. Definitely from my perspective having it right down the road from Ryerson, is amazing. (Participant 4, Student)

[I]t was pretty well laid out I think. I remember they had an elevator that was kind of unlike one I ever seen before. It was kind of like it, it's not like behind a wall, or door. It was just sort of there by the staircase. It seemed kind of cumbersome, to have to use it, or the way in which you had to use it, just seemed a bit, it was kind of like, weird and clunky. And I know a bunch of times it didn't quite work or they had to like call people 
to help have them figure it out how to use it. So it seemed kind of confusing. But I don't know like if that's the standard for that type of elevator. (Participant 7, Student)

So, they can have transportation covered. So I think, I don't think that becomes a barrier, as long as the patient makes it known, I think there's a lot of resources here, that can facilitate, most of those kinds of obstacles to receiving care. (Participant 3, Health Care Provider)

Collocation of services. The collocation of services was frequently described as an asset to improving access to patient care, facilitating interprofessional communication, and increasing referrals. Moreover, it was identified that collocation may provide a sense of reassurance and trust in the professionals collocated at St. Michael's Hospital FHTs.

Just the ease of being referred downstairs. Because it was attached, it was easy for them to refer, easy for me to go, easy to find. (Participant 17, Patient)

Even same day appointments. I notice as a [health care provider], that I sometimes see patients, they have been for a Psychology appointment and they have planned and followed up for a particular routine piece of care that they come to me for. So, that is nice for some people too. (Participant 15, Health Care Provider)

I think that the patients really appreciate that everything is happening in the same location. (Participant 1, Student)

And I think patients, because they are all from the family health team to begin with, have a sense of familiarity with it. I think a good comparator would be chiro. Chiropractic services are also located in the same building just downstairs, on the same floor actually as clinical psychology. And in that collocation, it provides our patients with a level of reassurance. It kind of gives it, your family doctor works with this person, it is not some random person, it is their colleague and they trust what is being done there. There is a shared chart and a shared record, it is part of the same thing. So collocation, the physical location being collocated with the family medicine team department, helps a lot.

(Participant 13, Health Care Provider)

\section{Addition of the PTC to the SMH FHTs.}

Positive addition/essential service. Participants reported that the PTC has been a positive addition to the St. Michael's Hospital FHTs. They noted that the PTC filled a gap in services offered at the St. Michael's Hospital FHTs and improves patients' access to essential, evidencebased psychological services (e.g. treatment, psychotherapy, diagnosing) that they may not 
otherwise have accessed. As well, the PTC was considered a unique and valuable asset, particularly in light of the long waitlist for community services and the fact that patients can access the services at no direct cost. Lastly, including the PTC in the services offered at the St. Michael's Hospital FHT appears to legitimize its services, thereby reducing stigma and increasing the likelihood of patients will access them.

It is just that thankfully psychological services are now available to them. Because otherwise my first question would be simply to any patient that is diagnosed with depression, the first question in management would be, do you have health coverage or do you not? Because if you don't have extended health coverage, you get medication. You do not have the opportunity to go and do the psychotherapy because you can't afford it. So now, when they have that option, I can say well, what would you like? And there are a lot, I would say the majority of patients say as long as they can get the time off work, I don't want to take medication. I don't want those side effects. Doctor, what are the side effects of psychotherapy? My answer to that is none. Right? So it is a bit of a shame in the old days when we didn't have that access. (Participant 13, Health Care Provider)

I think probably, if the clinic wasn't here, it probably would be a pretty big gap, in terms of the rest of the, kind of St. Mike's Family Health Team. We get a lot of referrals of people who are just coming from there GPs. Lots of anxiety and kind of depression coming up in appointments with their GPs and I think that the clinic provides... [a] place to refer those patients on to. The sense that I was getting, is that previously, some of these issues were having to be just managed by GPs, who, probably weren't able to see patients weekly or spend too much time with them. (Participant 5, Clinical Supervisor)

I think that, being able to, even indirectly refer patients with a relatively limited wait time. And with the significant obstacle of ease that would be encountered, outside of the setting, out of the way for people who really need it, and who would least be able to afford it, in the community, in terms of private care. I think that's really nice to see. It's nice to have, in terms of those comorbidities of patients that might have, someone to address them, so efficiently and effectively. Because otherwise in private practice, you can identify, you know, and find sort of psychological, social needs, and yet really be at a loss, especially for patients who couldn't afford it, what to do. And yet feel very badly that these very real needs are not being addressed. (Participant 3, Health Care Provider)

I think [the PTC is] addressing the...underserved mental health needs of those patients. So, I think there's a lot of mental health need amongst that population, specifically the one clinic. There are several St. Mike's clinics, but the one clinic that refers internally, I know referrals come from other sources as well, but that population has a lot of mental health needs that are going under addressed and I think...before the presence of Psychology they had Social Work, which was helpful, but I think it was limited in its 
availability. So they had...social workers and, you know, given that population there's a lot of need such as housing, homelessness, things like that. So, they were, kind of, overworked. So we're able to address, or Psychology is able to address some more of the, kind of, diagnosed problems that some of these individuals are suffering with. (Participant 9, Student)

I think in terms of stigma reduction, if you have a psychologist who is on staff just the same way a dietitian and a physician are on staff, I think it just opens up discussion around integrating mental health into a more mainstream health care delivery system. So, I think it's really important to have it presented to patients as a team and has everyone working together. And it's kind of like we're all equally legitimate members of the team. So, I think in terms of reducing stigma and actually increasing access to mental health services. It's probably one of the best things you can do. (Participant 1 , Student)

I know when [my physician] was referring me to the Psychology Clinic, he seemed very respectful, probably impressed about what they were doing. Like, he considered it a solution, so he definitely considered what they were doing helping the patients. (Participant 17, Patient)

Complements other services. The PTC was described as a complement, as well as alternative to, other St. Michael's Hospital and FHT services and treatments (e.g. medication). Participants reported that the services at the PTC add to the biopsychosocial and holistic model of care provided at St. Michael's Hospital. Moreover, respondents noted that the PTC helped facilitate coordination of care within St. Michael's Hospital and external community services. It was also suggested that engagement in services at the PTC may facilitate engagement in other St. Michael's Hospital FHT services (e.g. motivation for chiropractic services).

We do work in tandem, so there's times in which I will see a patient until they're stabilized enough to do that kind of treatment. Or they might, [the] psychology clinic might see a patient and say, "You know actually, you know we've done this, but you need more work on, we've targeted your social anxiety and now you should go and get some help around how to actualize that, like now with [name of health care provider] to be out in the world and do things." So, we do a lot of partnering. You know I, not everyone can fit the psychology clinic and not everyone should be in my service. So it's a really nice partnership... (Participant 11, Health Care Provider)

I think we spend a lot of time with clients, a lot more time than some of the other services, or almost all of the services offered there I would say. So, we just get to understand on a deeper level what is going with clients. Because we see weekly for an hour and we really kind of delve deeper into the issues that are going on for them. So, I 
think that complements what they are doing. For example, we don't give medication but we do other things, so we can work together in that way. And, I have seen a lot of clients who they don't want to be on medication, they want to get coping strategies to deal with what is going on. So, we can sort of work with the physicians or Social Work or whoever else to kind of provide more wraparound services, I guess. (Participant 12, Student)

Even things as small as, I had a couple of people who were going through chiro at the same time either consecutively or sequentially and individuals who were, like, experiencing depression and who were very anhedonic and low motivation, helping them deal with issues with low mood and motivation made them more receptive and more engaging... I'm willing to say that they were more engaged in the other treatment modalities they were receiving by nature of their mood improving. (Participant 2, Student)

I think the nice thing, there are lots of nice things about 80 Bond, but I think one of the nice things is that we can probably have a lot more physical, start to bring together the physical health with the mental health. So, I think the fact, I am just thinking of one client who had many health concerns, they may bring that up in the psychological context but was so easy to address it there because we had the physicians there. And what they were trying to work on we could be working on in tandem. (Participant 14, Clinical Supervisor)

And even in terms of coordinating community resources, so if we had people come in and then we were able to match them up with other community resources once our work was done with them. (Participant 1, Student)

High quality patient care at the PTC. Participants reported that patients at the PTC are receiving high quality, effective care and are satisfied with the care provided. They noted that students exhibited a strong commitment to quality care and were doing the best work possible given their level of training. Moreover, patients appear to be improving and feeling better as a result of care they received at the PTC. However, student participants relayed that the quality of treatment was dependent on case complexity and level of supervision. Specifically, they relayed that the PTC provides excellent treatment for patients with mood and anxiety disorders, but the quality was less certain for patients with more complex presentations. In relation to supervision, participants reported reduced confidence in the quality of care provided by students who have less supervisory oversight. 
I am really impressed with how things are going. So, I'm very pleased with the therapy and both the students I've worked with. I like them both. (Participant 17, Patient)

Yes, I have had very positive feedback from the patients that I care for that have received care in the department. I haven't, I mean, sometimes patients are challenged by the care, but that I don't see as a bad thing. And I have sufficient understanding of how

Psychology works to know that this is not just sitting and listening, this is about a plan of care that patients need to follow and sometimes they grumble about that. Or even refuse it. But that doesn't make me think that there is something wrong with what is actually happening with their care. (Participant 15, Health Care Provider)

Quite confident actually. I've been pretty surprised, even for a very first practicum placement, how pretty competent students come in...before even seeing their first client, they seem to, I don't know if it's good practical training prior to that point, or if it's just through the courses that they've been taking, or if they just, have good people [and] clinical skills. But, yeah, they're really hasn't been times that I would be hearing a student talking about a client, and me thinking, 'Oh I could done such a better job with this client.' I think when they struggle, it's been because the clients genuinely have been complex, or really severe, or have a lot of comorbidity. (Participant 5, Clinical Supervisor)

I think I am pretty confident but it would also depend on the problem. So, when it came to mood and anxiety related issues, I felt pretty confident. But then when I had, again I keep bringing up the substance abuse. I know motivational interviewing skills, but beyond that I didn't feel at all confident or competent enough to deal with somebody like that. Or like I had another patient with psychosis. Now I would feel confident. At the time I did not feel confident whatsoever. So, I think I feel confident in the CBT model and that treatment model, but I don't feel like it fits for all patients and not at all time periods. (Participant 16, Student)

I think in terms, so to answer your question accurately is that to treat certain generalist problems like mood and anxiety, we are very, very good. My confidence drops when we get to more complex things like PTSD, like eating disorders, like emotion dysregulation, self-harm, substance use, psychosis. It would, you know, I don't know if everyone is equally competent and comfortable doing that kind of work. But mood and anxiety? Boom! You're going to be fine. (Participant 2, Student)

I was somewhat concerned about things that other students were doing who were working with other supervisors. I thought some, it seemed like some people, there was less oversight. I'm not sure why they seemed to be working a bit more independently and making decisions that I wouldn't have been comfortable making. So, but I think that goes back to who you end up with as a supervisor. But, I felt like I was, I was comfortable with the services that I was delivering, but I wasn't necessarily comfortable with the services that other people were delivering. (Participant 1, Student) 
Long wait times. Individuals from each participant group reported that with the high demand for mental health services, there is a long waitlist for psychological treatment at the PTC since its opening. There are also long wait times between time of referral and stages of care (e.g. between referral and assessment, assessment and treatment). These long wait times were seen as a barrier to accessing services at the PTC.

Just the waitlist...I had some trouble because they called me three times and then nobody called me. And then there was, anyways, so it took me a while to get in and get the assessment. And then after the assessment, they put you on another waitlist to get into actually see someone. So, it was a bit of a wait, but it wasn't too bad. (Participant 17, Patient)

I think the biggest challenge out the gate, is just meeting the need. So the need far outweighs the ability to provide. (Participant 10, Clinical Supervisor)

And it can sometimes be a bit of an issue when we need to get patients in sooner rather than later because there is a wait list and there is limited capacity as well. (Participant 13, Health Care Provider)

I would say the biggest challenge would've been the wait list. So not getting people in, fast enough, and that just because we were limited by the number of people there, and number of clinicians there. And the number of referrals we were getting, so it was nice to be getting so many referrals, which showed that we were an important part of the team, and that everyone wanted us to help out their clients, as well. But that was one of the biggest challenges. (Participant 6, Student)

\section{Interprofessionalism}

Below are themes related to key elements of interprofessionalism, including a culture of respect, hierarchy versus team-based care, and scope of practice. Along with this, participants described that interprofessional interactions and patient care at the FHT and PTC occurred more on a case by case basis rather more regularly. Communication, similarly, was seen as lacking particularly regarding direct communication among professionals from different disciplines. A lack of time was identified as a barrier to further interprofessional interactions and the level of interprofessionalism appears to vary based on individual and profession-specific factors. 


\section{Perspectives on interprofessionalism and teamwork.}

Culture of respect, openness, and equality. Overall, participants reported equality among the varying professionals, including students. They described a culture of respect, positivity, and openness to differing professionals' perspectives. Participants noted that most, though not all, health care providers and students had an open door policy and/or attempted to be available for consultation or knowledge sharing if requested. Some conflict was described, though participants stated it was limited in nature (e.g. some health care providers did not appear to value the addition of Psychology).

I love being down here. I love working with the students. I love working with [name of clinical supervisor]. I don't even want to switch to another clinic because I love being in this environment. I love it. (Participant 11, Health Care Provider)

At St. Mike's, I thought, I felt very respected. And I think that the supervision that I had and the guidance that I had and the modelling from supervisors and other more permanent members of the team, respect was always number one. So, I think, yeah, I think that they did a good job of trying to make everyone as equal as possible. And at the very least, respecting everyone's opinion and recognizing that people have different training and different experiences that can all help improve patient care. (Participant 1, Student)

Again, I think I sort of have a lot of experience with respectful communication between providers, social workers, physicians, [the] dentist. There have been sort of rare instances where the opposite is true and that's been unfortunate, but overall, I think it's a very respectful environment to work in. (Participant 8 , Student)

One small example is just me having written a note to a doctor because I needed him to make a referral to addiction and then he wrote back and just kind of said that was a great note, thank you for sending it along, and did what he needed to do and got the client referred to where they needed to go. It is a really small example but I thought it was a really nice response to the message that I had written. And then it got done and the client got what they needed (Participant 12, Student)

Limited hierarchy experienced. Although some participants denied experiencing any

hierarchy or experienced a flattened hierarchy within the FHTs, others reported a hierarchy with the physicians on top. Some described how this may be an artifact of the original single 
physician provider model, while others remarked that it was necessary for one profession or

individual to take on a leadership role for effective team-based care and administrative purposes.

Definitely there's team-based and, people really do collaborate, I mean, with, you know, hallway consults and all kinds of things like that, importantly. And, I haven't really seen any attitude or anything, but, definitely because we're in a hospital setting, there is a hierarchy because of the hospital set, that governs disciplines in the hospital, there's the hierarchy, medicine on the top, in terms of the administrative structure, and, sort of the committees, the health disciplines committees. So, there's hierarchy that way, so, doctors, nurses, the rest of us. (Participant 3, Health Care Provider)

I think, like, kind of... a bit of a hierarchy. I definitely see like the physicians would be on top. I don't think that they are ever, like, differential to the psychologists, all that much... So, there weren't like, you know, questions about like, how do things work, or what you do. It's like oh, this person seems depressed, and I'm not going to do therapy, so here, we'll just get the psychologist to versus psychologists, we're never, you know, never like assumed things about physical health problems. So, yeah there seemed to be a hierarchy there. And it's not even like the, physicians on doing, like, it's just sort of even the way, patients are, or administrative staff. Even you know, like the fact that there on the top floor and you have to like go down to the basement to, you know, see the other people, that kind of stuff. (Participant 7, Student)

So there's a sense that we each have our own purview and - but there always is going to be somewhat of a hierarchy and I don't necessarily feel badly about that. Like I don't have an inferiority complex. Psychiatry is doctors who got an advanced degree. I defer to them quite often for their opinion and viewpoint on treatment as I would to [clinical supervisor]. Like, I consider her someone who has more training and more experience in various areas... The doctors, I defer to the doctor. They're the physicians who kind of manage patient flow. So I mean, I know hierarchy has a bad connotation, but I'm not sure flat organizations structures completely acknowledge sort of expertise and difference. And I don't think there's, as long as there's mutual respect I don't think there's anything wrong with that, you know. (Participant 11, Health Care Provider)

Yeah. I think they tried really hard to have a team-based approach. In my personal experience, I never felt like I was lower or higher than anybody else. So, I think that the St. Mike's Family Health Teams are doing kind of the best job that they can, but there is a sense of, you know the physician has the final say. (Participant 1, Student)

I think that there is a hierarchy of professionals within our health care system. I think our health care system is still very medical model, but that said, in the grand scheme of where you fall on that continuum of medical model, I find just based on my experience that St. Mikes is probably one of the most open, flexible systems that I have worked in. Where I feel that there is certainly a medical model, where physicians are above other professionals and they are sort of the leaders of the hospital, is sort of how I have seen it. But that said, there is a great openness and great interest in hearing from other 
professions and certainly psychology. And I have and my students have been treated with a lot of respect. But, you know, I think there is still a medical model. (Participant 14, Clinical Supervisor)

Scope of practice. Participants reported that professional roles and responsibilities were initially less defined but were becoming more established over time. They stated that when overlap occurs or conflicting professional approaches arise, it is discussed and managed so that each team member is focused on a unique issue or treatment modality (e.g. medication and psychotherapy). However, some noted that duplication of services, conflicting approaches, and role confusion continue to occur.

When I came to [the SMH FHT/PTC], very few referrals came to Social Work, everything was being given to Psychology. And sometimes I would read something and this is clear adjustment difficulty and why is it being sent to Psychology? This person needs to talk about their life and have some strategies and do some grounding, et cetera, et cetera. It made no sense to go through a diagnostic interview and then have treatment for 10 weeks. And so [the clinic supervisor] completely got that and so having a centralized model at the clinic. And it helps them. It didn't help Psychology before to be everything and everyone to the department. It actually undermines the clinic. So, [the clinical supervisor] is very clear on what the clinic does, what they do well, and how to facilitate good training experience for the students which is a big piece of the clinic. (Participant 11, Health Care Provider)

So Psychiatry, mostly they're consulting, it's not to do treatment but to do diagnoses. So sometimes yeah, there is an overlap with the types of diagnoses that they were providing, and that sort of thing. But, we really attempted to catch it, so that they're not providing a service that we can actually do, or that they're not doing a diagnosis at the same time as...down the road like, you know, if they made a referral to us that, then we would do another diagnosis. So not wasting resources, so we're trying more vigilant on that. I think with therapy we do overlap a bit. But again, it's just different training. (Participant 11, Clinical Supervisor)

I mean, if there was going to be duplication of services I would say it would be between Social Work and Psychology, if anything. But even there, I mean I know for myself, I often, if I am going to see a client that has been referred from Social Work, then I will speak with [them]... and just try and figure out what have they done, what are they doing now. I know [name of clinical supervisor] makes a big effort to encourage us to read through a file and have a good understanding of everything that the client is receiving at that time, so that we are not doubling up and we are not overloading them and we are not putting them in a position where they are doing multiple treatments and it can be confusing. So, I don't see a lot of duplication of services, but I do see efforts made to 
avoid that from happening, which is good because there is a huge waitlist and we don't want to be duplicating the services when we could provide it to someone else who needs it. (Participant 12, Student)

I think having clarity in terms of...roles and responsibilities is a really important one, too. We have had instances where doctors were doing psychotherapy at the same time that we were trying to do therapy. And there was a need to have a discussion about that and talk about how it was all done with the best intentions for the patient without a doubt, but that what it actually was resulting was different messages and that that could potentially be confusing for a client. So again, having clarity in terms of who is going to handle what parts of it. And if you are going to be doing some of the psychotherapy, maybe it is limited to this piece of things, whereas all things having to do with this piece are the responsibility of the psych clinic, for example. So I think that there always needs to be clarity in terms of who is doing what. And the other is what the expectation should be in terms of length of service. (Participant 14, Clinical Supervisor)

Case by case interprofessional teamwork. Overall, participants described

interprofessional teamwork that occurred on a case by case basis rather than integrated teambased patient care. For many, it appeared that patient care was conducted separately and each profession worked in relative isolation. However, some participants described a high level of interprofessional teamwork. Yet, across participant groups, respondents noted that there were limited opportunities for interprofessional team meetings to discuss case collaboration, treatment planning, or informal discussions about shared patients' care. Overall, participants wanted more structured opportunities for interprofessional teamwork and recommended more team meetings to more fully engage in team-base care and provide an opportunity to learn about other professions.

So, we work in a building that has like a dentist on site, a social worker, the physicians are upstairs, so there's opportunity to collaborate on patient care if it comes up. But, there's no sort of structured format for that. So there's no, sort of collaborative care team that you're just sort of walking into. You have to sort of learn how to navigate it. On a case by case basis from the start, which has been challenging, but sort of rewarding I guess at the same time. (Participant 8 , Student)

I just think there wasn't that structure set up where they do have... like I don't know if they had, if the doctors had meetings. I am assuming they did amongst themselves...We never really had meetings among the different professions together. So, if you don't have 
that structure or organization put into place as a foundation, you know what I mean? Then I wouldn't be, like doctor so and so, and this nurse, can we meet? It is not in our schedule. And, who am I to kind of impose that on them? If that makes sense. It wasn't built in. (Participant 16, Student)

Yeah, so in addition to just having common areas, you also need scheduled times where people meet. So we have these lunch and learns where we do interprofessional education. We have team meetings where people are mixed again. What we did early on, and we might need to reintroduce this again at some point, but what we did early on was we actually had team meetings were lunch was provided. So, even further extend that idea of that dining room table, right. You gather everyone, everyone is eating, they are talking. Sometimes it is around clinic related stuff, sometimes it is not. (Participant 13, Health Care Provider)

So we have, where we meet weekly as a team, the Psychiatry, Social Work, and myself, and we triage, and discuss each of the referrals, and make decisions about how we either collaboratively or individually will take those referrals. And that's actually been just piloted here, at one of the sites. And we found it, it to be a really effective way of conducting services, cause often times residents, and sometimes physicians don't always know, and it can take up time, you know back and forth, discussing who's the best resource. So, we found that to be really beneficial. (Participant 10, Clinical Supervisor)

Limited direct interprofessional communication. Overwhelmingly, participants reported that interprofessional communication was underutilized overall. They stated that it was limited in nature, less than expected, and needed improvement. Participants noted that more person-toperson communication was preferred, including either over the phone or more desirable, face-toface. It was noted that the electronic medical record (EMR) was the most frequently used form of communication and it facilitated communication overall. However, some felt there was an over-reliance on the EMR as the sole mode of communication. On the other hand, given the time constraints health care providers often face and varying work schedules, the EMR was seen as an asset to initiating and maintaining contact, due in part to its email and instant messaging features.

So, I was just thinking about like most of the communication was just, you know, like saying 'hi' in the hallways, you didn't really get a sense of like who was doing what, and because it was a training facility, like the students were always changing, so that made it more difficult, I think, to make these connections. So yeah, the communication was just 
like, 'oh if there was something that this person needs that might be better served by this other department,' then, then we'll, you know, send an email. And that would be the extent of things. (Participant 7, Student)

I think kind, I think openness of communication would have improved, and thus the lack of it had, you know, hindered, you know, team-based. There wasn't a lot, like it was a team in the trivial sense of the word. Like we're all treating the same person and occasionally we talk to each other. (Participant 2, Student)

Yeah, I think [communication is] a very important part of patient care. Based on my experience there, one that was underutilized or occurred less commonly than we had hoped or been told it would occur. I don't know how things have changed. (Participant 9, Student)

There isn't enough inter-play between physicians and nurses. There is not enough direct communication and discussion about the care of individuals that the physician or the nurse have concerns about. We perhaps over-rely on the EMR. And we really [need] more in-person collaboration and just friendly communication on a person to person basis. That is the way you build trust and a true team. So we need to still move towards that. (Participant 15, Health Care Provider)

Being able to message through the EMR, being able to call and say, oh you are in your office, great I am going to pop up. That has made a big difference. I think what is more difficult is when students are trying to reach people that are not on site and they are not in the clinic. And so it is very hard because they are not there to answer their phone, they are there one day a week. But you know, I think over the couple of years that I have been there I think we have managed to find ways to use reception, to leave very detailed notes if this person calls, book a time to have this telephone call, so that we can work around some of that. But again, it has taken coordination and it takes good communication within our system as well as outside the system as well. (Participant 14, Clinical Supervisor)

So, it is so important for the GP who referred a client to me to know what their client is doing with me. I often had clients come in, and would say, oh yeah, so and so, my doctor mentioned that, they were so proud that I was doing this treatment with you, because they can see the notes in the EMR. So, that not only made the client feel good, it strengthened their relationship with their GP. And, often strengthened their commitment to therapy with me. So, that was really good, it was so good for the GPs to know what we're doing with the clients and show them that we're, working as hard as we can to help their clients make progress in this specific area of their life. I also think that it's good on the flip side, for me to see what is going on with them, and their GP. Because if they're having like, meds changes, that's important for me to know about, because, well, if their depression symptoms increase that week, then I can know, oh maybe it's cause their working on their meds. And we can talk about that. So, I mean the EMR was invaluable. (Participant 6, Student) 
Level of interprofessionalism is person/discipline-driven. Participants described how the level of interprofessional engagement was dependent on the profession, individual characteristics, level of leadership, and level of training. For example, a strong interprofessional relationship was reported between Psychology and Social Work and weak relationship with Psychiatry. For the most part, a positive relationship between Psychology and Physicians was described, though it depended on the physician with newer physicians seeming more receptive to Psychology. Interactions with Chiropractic, Dietetics, Dentistry, and Nursing were noted as generally positive, though limited in frequency. As well, some participants described actively seeking interprofessionalism, while others noting that it was not a priority, suggesting that there are individual differences in a desire to engage with other professions. This may be due in part to level of training; individuals who identified as past versus present upper level student participants appeared to be more inclined to engage interprofessionally. Lastly, those who reported holding leadership positions appeared to be more engaged interprofessionally than others.

So we have a lot of contact with the physicians, the primary care physicians and the social workers, but not, I don't think with psychiatrists. I've never talked to one in terms of patient care. (Participant 8, Student)

I mean like I worked a lot with a social worker who [worked] with trans people...that was actually just helpful for me and my training. (Participant 7, Student)

And you know, slowly integrating us in to, you know, other aspects of treatment and doing collaborations, like with co-facilitating groups with dietitians, and you know, working with Social Work in a variety of different ways, on presentations, professional presentations, and things like that. (Participant 10, Clinical Supervisor)

You get people kind of comfortable in their own skins and comfortable talking with each other so that it is not intimidating for someone to come up and talk to me, for example. I don't consider myself a particularly intimidating person, but if you've never met me and you don't know who I am, only seen my name and the various random titles that I have collected, then sure it might, uh who knows who I am? But because everyone in the 
clinic knows each other it is much easier and things function fairly smoothly. (Participant 13, Health Care Provider)

I think in terms of our role we probably more took the side or were more passive, and because we were also students we didn't want to overstep our boundaries. We felt, well I felt, that it was really up to our supervisor to not only show us the way but to make us known in the clinic to other professions. (Participant 16, Student)

I don't know if, and I also think it depends, really on the student and the patients, and the referring physicians. Because I didn't have a lot of communication with other disciplines, but I know that another student in my cohort worked with a dietitian or a registered nutritionist on an eating group, so she had a lot more experience with that discipline. Another student felt, that they possibly needed to form a patient, so they had a lot of communication with the patient's physician because patient was just very suicidal, so they talked a lot to that person's family doctor. But, so I think it really depends on the student's experience or how much interaction they seek out with the physicians. I just didn't happen to need to do that very often, so maybe my knowledge is more limited than someone else's. (Participant 4, Student)

And I think that, you know, the other thing about the clinic is that you can always just call up a doctor, or walk upstairs and grab them, and people were generally pretty open to that. And that is great. If I had a quick question for someone, then it's nice to not have to go through a whole email process or to wait on responses when I can just pick up a phone and call them really quickly. And I can see their schedule in the EMR, so I would know if they were free. So it's even better. (Participant 6, Student)

I felt that Psychology was, at least, in terms of our students, were playing more of an active role and reaching out to residents and the GPs than was the reverse. It was very rare that a GP came down and talked to me because they saw something. (Participant 2, Student)

Lack of time. A lack of time was described by many participants as a barrier for interprofessional engagement. They noted that although there were some interprofessional activities, there was limited time to attend those that were available or reach out to other members of the FHT.

So, one of the things that I noticed from working in this environment is just, how busy everybody is, and how many meetings they have or don't have. And you know, they're training their students, they're also seeing a high case load. And then most of them, you know, there's a core group of physicians at each clinic that are full-time and then most of them are part time. So they have multiple jobs, so they're consulting too, you know, may be two hospitals, or two settings, or three settings, in a week. And, so, and in that sense, I think it's the meetings, it's the opportunity to do team building through meeting and 
discussing cases, current cases, where everybody, you know, where there are multiple people involved. So, you know, [FHT/PTC clinic] does not seek that out, like they don-, we've tried it, and, the docs don't want to, cause it's just an added meeting. Whereas, other sites do seek that out on occasion. You know, once a month they might have meetings and they discuss current clients and Psychology and Psychiatry are at the table to give their input or answer questions or things like that. But at [FHT/PTC] clinic they don't...do that. (Participant 10, Clinical Supervisor)

Yeah, I mean we were encouraged to always go. Like I think it was every month they would have a grand rounds or something that we were always invited to go to, but just given how many things, how many directions we're being pulled in. And, a lot of us were already spending more time at the clinic than we were supposed to be that those extra things to integrate us into the team just kind of didn't make the cut. (Participant 1, Student)

But I think that the other thing that is very, very helpful is that the load, even with one day a week, the load is very heavy for students. There is a lot of pressure for students to put in hours. And the number of clients that they see is quite a lot for a first practicum, in fact. And I think that they do very well. And as supervisors we were working an inordinate number of hours. Like many more hours than really what we should be allocating to it. (Participant 14, Clinical Supervisor)

So, a few of us were able to, for example, actually talk about interprofessional collaboration and teach other student learners about the role of Psychology in a, sort of as a part of a workshop series. And, they do hold those types of workshops put on by sort of different people within the hospital for student learners I think on a monthly basis. And our director makes sure to send us those emails out and encourage us to attend. But it's not always possible. But, beyond that, and I'm not, yeah, there have been, sort of limited opportunity to sit in on clinical rounds and things like that, but beyond that. (Participant 8 , Student)

I think everybody's busy, so to have formal meetings, doesn't really work well. (Participant 3, Health Care Provider)

Proximity. It appears that proximity may be a moderating factor in relation to collocation and interprofessional communication. For example, participants who were around when the PTC first opened reported that when the PTC was housed on the same floor as nurses and physicians, interprofessionalism was higher and decreased when the PTC moved downstairs. As well, participants noted that interactions were higher with the social worker, whose room is along the 
same corridor as the PTC. Lastly, interprofessionalism was viewed as higher at the FHT where the PTC is collocated and less at the other FHTs.

And I think the proximity of Social Work being located in our hallway, makes us use Social Work more. I think if the physicians were located in our hall way it might be different. See, like I am talking now about the locations which I wouldn't have done otherwise. But I think that physical proximity and collocation breeds communication. And communication helps with integration and providing holistic service which is going to improve our clients overall well-being. (Participant 14, Clinical Supervisor)

I think that [when] you've got many different disciplines together makes it's easier to ask questions. One thing that I think is not so good is that the physicians are upstairs and Psychology is downstairs. I think, when I was there, we were all upstairs even though we were seeing patients in examination rooms, which wasn't so great. At least if you had a question or you wanted to interact with a doctor or a nurse, a physician or a nurse, you could do that really easily by just knocking on someone's door or asking a quick question, you get information about medication. I think that since the division of building the downstairs, even though it's very nice and comforting for patients, we've lost that ability to work as an integrated team that existed before. (Participant 9, Student)

So, definitely I think ideally, it would be wonderful to have a centre that is like circular in nature or all on one level. Because I just think by that, very nature, it kind of, there, it creates a hierarchy, in and of itself. You know, the physicians are upstairs, and we're downstairs, and, so just the opportunity to have hallways conversations is really limited. (Participant 10, Clinical Supervisor)

The geographic layout can sometimes help. If everything were on the same floor then there would be more circulation, more familiarity. That we do try to break down to some extent by having meetings downstairs in the meeting rooms. (Participant 15, Health Care Provider)

\section{Clinical Psychology Graduate Student Training}

These themes pertain specifically to the training of clinical psychology graduate students at the PTC. Benefits of the PTC as a training clinic are reviewed, including positive learning experience, integration of evidence-based learning, clinical leadership, resources, and experiential FHT and interprofessional learning. Drawbacks are also described, including lack of specific FHT and interprofessional training, as well as other general training limitations. 
Participants outlined positive and negative supervisory experiences and the impact it has on the training experience, along with the benefit of having different supervision modalities.

\section{Benefits of student training at the PTC.}

Positive learning experience overall. Overall, the clinical practicum at the PTC was described as a positive learning experience and participants were satisfied with the training they

received. Respondents stated that the training was supportive, gives an edge over other practica, and well-prepares them for future practica and internship. Some noted that training was developmentally appropriate and tailored to students' learning goals, though this sentiment was not shared by all participants. As well, respondents overwhelmingly endorsed the diverse patient population as a key benefit of the experience.

So, well personally that experience really filled the niche for me and filled a gap in my training that I had been missing. And, it was, it gave some really good experiences knowing now that that's something that I want to work in. I like that team approach. I like having people with different expertise coming together, to focus care on one person from multiple perspectives. And, without being there, I might not have known that. And that really shaped where I, like decided to do my future training, so where I decided to apply for internship, where I ended up, ranking and matching. So, that experience was really integral to what I'll be doing in my future. (Participant 6, Student)

Ah yeah, so I think it's really helped in terms of getting my current and subsequent practicum experiences. The, just the amount of diversity that you see, so in terms of culture, in terms of, you know, socioeconomic presentat - different presentations of socioeconomic status, differences in, like, diagnostic presentations, I think the diversity is really one of its strongest features and really helps in starting a foundation and also getting other experiences in the future. (Participant 1, Student)

I think it was really good to start off there. It was my first practicum. Because it wasn't, they were relatively diverse in terms of presentation and complexity cases. So most of the people there, you know, although they had usually some sort of mood or anxiety complaint, had complex histories with varying [socioeconomic status] because it was an urban centre team by people who worked on Bay St. and people who were had [Ontario Disability Support Program]. You know people, I had someone who was...17 and someone who was 66. You know, I saw someone who identified as trans and, you know, everything in between, someone who was coming out and now identifying as gay, every-So I think in terms of the diversity, you know... it was a firm generalist practice. (Participant 2, Student) 
It's a very supportive environment. Like I did not feel anxious, well I felt anxious before going there, but like right off the bat, it was very clear that, you now, it was going to be a supportive learning environment and I really appreciated that. Yeah, I think that's maybe probably the most important thing. (Participant 8 , Student)

Again, thinking about that developmental progression, to be able to give them what they need to go out. And I think when they go out it speaks in terms of practicum matches and our students are doing really well. Internship students are doing really well. And I think we are being recognized that our students, they have seen a lot, they have done a lot, and it has both been experiential in the clinic and in terms of course work. I think the two together are making our students some of the best-prepared clinicians. (Participant 14, Clinical Supervisor)

Integration of evidence-based learning. Participants remarked that the student training at the PTC complements classroom learning and provides a good foundation of knowledge. They noted that they gained exposure to working with patients with mental illness, experience assessing and providing DSM 5 diagnoses, and increasing expertise in providing evidence-based treatment.

I think it's a really unique training opportunity. It's, I think it also sort of really complements what we're learning in our courses. So we can literally, sort of take that theoretical knowledge and apply it in this like practicum that they've set up for us. You know, we're being trained by people who have years of experience in empirically supported treatments. So, I think it's amazing training. (Participant 8, Student)

I think from an experiential teaching perspective, I think it gives us the opportunity to take what we are teaching in our courses and bring to life in the clinic. We don't know when people go off and they do a practicum. I mean we know many of our sites, and many excellent sites, we are very, very fortunate, but this is a way that we can actuallywe know what they are learning in class and we can tie what they are learning in class to what they are doing in the clinic in a way that when you go to a practicum you can't. So I think that and vice versa, when I am teaching. So, when I teach graduate courses that are in treatment, I will say for example, at St. Mikes, dah-dah-dah. Let's tie that to what we are talking about. And that is something that I couldn't do unless I was involved in both sites. (Participant 14, Clinical Supervisor)

I think both the combination of the supervision and then when the courses are offered,... this is one class, but I think this was the first year the class had CBT course before they, in their first semester...[w] hich I think really helped. So, you know, the students who were running the group with me, and then some of the individual treatments, and then, just their ability to take, now, a manual, the diagnosis, the person 
that's sitting in front of you and really flush out a good conceptualization. And, I, you just see it's really, I think facilitated that growth, and it's timed really nice. So, I'm hoping that that will stay 'cause I think it's a really good combination, so their second year, their first semester, they have the CBT. (Participant 10, Clinical Supervisor)

Clinical leadership. Participants praised the clinical supervisors for their open door policy, availability, interprofessional modelling, and encouragement of interprofessional collaboration. They noted that this provided positive scaffolding for working in an interprofessional environment. However, some stated that the competing roles of clinical supervisors did at times interfere with optimal training, particularly since there was only one fulltime psychologist on staff in the FHTs until recently.

I think it is something that [the clinic director] does a lot and she encourages us to do it even if it just means sending a note to the physician to say, I am going to start seeing your client just to let you know. So kind of opening up the lines of communication in that respect even with something small. So she does definitely encourage us to do that. I think it is about for us learning how do you do that. Not only is this our first practicum, but we don't really know what is OK and not OK do to, or where we need to be opening up the lines of communication. So we do look to [the clinic director], or supervisors in general to kind of tell us when it might be good to check in with someone else. (Participant 12, Student)

I would say the relationship between the clinic director and the social worker at our site was a good model of how to build an interprofessional team approach. Like they, I know they consulted a lot on cases and even outside of professional discussions, they just were, seemed like a really good team and really respectful of each other's work. So, I think that they were a good model for us of how to really take advantage of the other people on your team. (Participant 1, Student)

I know that I feel that the director has an open door policy. In fact, I know [the clinic director] does. And if I had a specific question I could make an appointment, I could message her, email [the clinical supervisor]. [The clinical supervisor] is visible and very accessible, friendly person, and I could always go and discuss something if I wished with her. I know [the clinic director] would welcome that. So, that is as much as I need at the moment. (Participant 15, Health Care Provider)

I have never felt like my input or ideas where dismissed. [The clinical supervisor] is very honest and direct, so if [the clinic director] has an issue over something, [the clinical supervisor] will say it. [The clinical supervisor] will be very clear about what [the clinical supervisor] wants and what is good for [the] students... I think we are lucky. (Participant 11, Health Care Provider) 
I think just kind of having, I mean I know at the time my supervisor just had so many competing demands, so I can understand when, you know, conference calls have to happen, or if I need to talk, you know, other people can't always be at my convenience. So I completely understand that, but I think just maybe having someone whose, a little more readily available for consultation, would be helpful. But, I mean I understand that there are limits to that. But, that would be really my only, real, I guess, point of, and area of improvement, would just be availability of supervisors. (Participant 4, Student)

Resources. Many respondents reported that the number of resources, the library, and audio-visual recording for supervision were benefits to student training at the PTC. However, a few participants stated that more materials (e.g. manuals, assessment measures) were needed.

I think that they have really good resources here, for student learning and for supervision. So, having really nice therapy rooms, that are pretty uplifting and modern, having the capacity to video tape sessions, and to watch them like on a computer, during supervision sessions, is really helpful. All kinds of training resources: books available, [the] library, having group supervision with the students, different workshops that they can go to, clinical rounds. So, I think it's pretty top-notch, as a clinical training environment. (Participant 5, Clinical Supervisor)

We had a very big library. Well at the time I didn't think it was very big, but now I do. Big library of materials, so it would be very easy for me to look through that library and find information on problems that I needed or if I had a question that was right there. There was also a digital library. Supervision was, overall, quite good and often, and like the use of tape recording or video recording, it was helpful to receive information. (Participant 2, Student)

I think that also that the rooms are equipped with the audio and visual recording is really important. I found that incredibly helpful, especially being our first practicum, you can really see all the ins and outs of what you're doing and so I think the actual facility is really good for training purposes. (Participant 1, Student)

I think having more manuals at the clinic would be really helpful. (Participant 8 , Student)

I don't remember there being, I didn't do that many assessments so I don't remember ever having a problem with not access to an assessment tool. But I do remember some other students at the time wanting certain tools that were pretty basic that they didn't have access to. But then having access to tools that were not really needed. I think for example the PCL-R, which is the psychopathy checklist, for some reason that stands out. Like I use that in my forensic practice, but you wouldn't really be administering that in a hospital, general mental health setting. So that one stands out. But I personally didn't have any issues, I thought there was appropriate or good access to whatever resources I needed. (Participant 16, Student) 
Experiential learning about FHTs and interprofessionalism. Respondents reported

being comfortable with the training model as is and feel like it facilitates experiential learning about FHTs and interprofessionalism (e.g. gain exposure to other professions, general understanding of FHTs). Health care providers and clinical supervisors, as well as students, described the benefits from learning about the different scopes of practice and the FHT model experientially.

Yes, I think going in, we don't necessarily understand the roles of each department. But, through the experience working there, and through some of the trainings, and the didactics that are given to the student there, we can learn and better understand what specifically Social Work does, what specifically the dietitians do, what specifically the GP's do, even though that's a bit more obvious. And I think, it's part of the training. So going in, I don't think we have the knowledge, coming out, I think we do. (Participant 6, Student)

So, I think that it helps in their training here, by giving them a better sense of the scope of health disciplines where there's overlap for certain problems. By overlap, I mean... like back pain, [a] medical physician would possibly take the medicinal route and take the physical route. And, also how co-morbidities are managed, again, by various disciplines, what there in a discipline, for psychosis, by the family practice doctors who might also be referring patients for Social Work or psychological intervention, here, at the same time. So, I think that way it's very helpful and probably done more effectively than if they were to sit and read about, this is the scope of practice of medicine, and here's someone with neuros-, or psychosis, and here's how they can be managed, or something. I think works better that way, informally though, so maybe not completely. (Participant 3, Health Care Provider)

Besides just interacting directly with clients, I think it has been beneficial to work closely with other health care providers. I hope I get the opportunity to do that at my next practicum, but it's been like a pretty good introduction, I think. And I've learned a little bit about, I guess, what other professions do. So I've also worked with a dietitian and sort of have a general sense I guess of what they do with their clients. And I learned a lot more about Social Work. (Participant 8, Student)

I think just being aware of different perspectives of things. So, maybe the way we do a group is a little bit different from how other professionals might do groups, recognizing that many of the issues that we see here, are multi-factorial, so it's not, sort of, just a Psychology issue, but sometimes it can impact physical health and diet and lifestyle and all those other things. So, I think it gives you a bigger perspective that many of the issues 
that we see really have different contributing factors. And for that reason, many different people have a role in treating them. (Participant 5, Clinical Supervisor)

I think that for myself personally what I have been able to gather from the past several years of having clinical psychology co-located with us is really that deeper sense of appreciation of what clinical psychologists actually do. Let's say if we rewound six, seven years, I don't know that I would have seen any significant difference between what Psychology does and what Social Work does. Psychiatry I would understand, only because from that medical model I rotated through Psychiatry, I have seen what they do, I have experienced it, so that makes sense. But the challenging diagnosis, that is where it has really helped. And I don't know that I would have been previously looking to Psychology for the really challenging mixed diagnoses. Your garden-variety depression and anxiety are fairly straightforward, and in fact declare themselves. Most of the patients know their diagnoses already. But so when you start mixing those things around with a whole bunch of problems like social factors. Are they having difficulty adjusting or is there a mix of a little depression, a little anxiety, some psychoses? It is the difficult cases that really make the case for bringing someone else in. It makes the case for understanding where your own shortcomings are. (Participant 13, Health Care Provider)

\section{Drawbacks with student training at the PTC.}

Lack of specific FHT/interprofessional training. Throughout the interviews, student participants conveyed and reported a lack of knowledge about the FHT model, the full range of services provided at the SMH FHTs, and interprofessionalism, in general. This was in contrast to the more knowledgeable health care provider and PTC clinical supervisor participants who exhibited greater knowledge and understanding. However, this ranged on a continuum with some participants who were more knowledgeable than others. Participants reported that they would have liked more FHT and interprofessional-specific training, increased modeling in this area, and greater opportunities to connect with other professionals.

I would say moderately knowledgeable. I would like to be more knowledgeable. I don't think it's ever been explained to me as a model for care. I think it's more like, 'we have all these services' as opposed to 'this is, kind of like, the theory and model of a family practice and why we think it's more either cost effective or better for care.' I don't think that's ever been, like it seems logical as to why it would be better, but I've never seen that kind of laid out. So, I would even say maybe less than moderately knowledgeable and comfortable. I would say about the same. I'm very open to it. I think it's fan-like it's great. But, more knowledge and more comfort in like how things are conducted day to day would be helpful. (Participant 9, Student) 
So what is the family health team? Is there a model? (Participant 16, Student)

I never thought of that. I mean we did, there was like generic didactic training about the nature of the facility in which we operate. There was no process instruction about how that could influence our practice...I mean there is indirectly in terms of like, how you make referrals, what kinds of problems you speak to these individuals about. That was kind of haphazard...There wasn't, I mean there wasn't a day, again, clear flowchart about how certain things function. (Participant 2, Student)

I wouldn't say that I am that knowledgeable. Other than just what I see when I am there. But beyond just kind of knowing that there are a lot of different services housed in the same place, that we are able to try to work together to support clients, like I don't know a lot more about it. (Participant 12, Student)

No, I think that was a bit of like learning as you go along. But again, like supervision sessions we were reminded, or at least I was, of all the different people that were working there. And how we can think about how they might be used. But it could have been helpful, actually, if we did have, something early on that, wer- someone and came and talked about, like this is what this department supposed to do, this is what they're supposed to do, and so on. So, we just like had a better sense and then maybe like with some cases, so when this happens, you'd refer here, and here and there and this is how you go about doing so. There was a bit of, just sort of figuring things out or asking, or like do I even, do I call for this, do I email, can I just walked into this person's office? Like, what's kind of the social etiquette around that stuff? You just had to like, pick it up as we went along, so maybe a little, little, I don't know, seminar on that, early on would have been helpful. (Participant 7, Student)

Limitations of training opportunities provided at the PTC. Participants noted that there were limited opportunities beyond psychodiagnostic assessment and individual CBT-based psychotherapy. Student respondents reflected that although there is an opportunity to gain child assessment experience, they would have liked to gain experience administering comprehensive, psychoeducational, or Axis II assessments, as well regular group psychotherapy, which was not available to all students. Also, some participants noted that the generalist training and focus on CBT for adult mood and anxiety at this mandated first practicum was a mismatch with their long-term goals to work with children, families, forensic populations, and practice in other treatment modalities (e.g. emotion-focused therapy, interpersonal therapy). 
I don't know that this is a challenge, maybe a drawback. I want to work with children and families. This is an adult intervention. So I know for me, it is not exactly what I see myself doing in the future, but I still think it is incredibly beneficial to have been there. (Participant 12, Student)

Even though I didn't really want to do treatment, at least now I have a sense of, you know, what CBT is, and what other interventions are. But for, I don't know, people who really just want to do child work, for instance, which you can do some child-based psychoassessments, at least you could when I was there. It might just sort of feel like, 'oh I'm doing all this work, and it's not for not, but I'd rather be spending my time, you know, seeing children,' for instance. So, that might be a challenge for some people. (Participant 4, Student)

I probably would have liked to do more assessment because I found it be more treatment focused. But I think that is just the nature of the setting, you don't have all the time in the world to do this comprehensive assessment like you would like to. (Participant 16, Student)

One thing that I was hoping to get out of my training that I didn't end up getting was different assessment experiences. So, it was kind of presented to us at the beginning that if you wanted to do a more comprehensive, like educational assessment then that would be an opportunity and that opportunity wasn't really presented 'til, to me until the very end and then we couldn't get in touch with the family, so I wasn't actually able to have that experience. So I think one drawback is maybe more things are promised than can actually be executed. So, that was, yeah was something that was disappointing about it. (Participant 1, Student)

\section{Clinical supervision.}

Mixed quality of individual supervision. Supervision was described as a key contributing factor to a positive or negative training experience. Some participants reported having excellent supervision, while others reported poor supervision (e.g. checking emails in supervision, not present/on site, lack of structure, not enough oversight). Even students who had a positive supervisory experience reported dissatisfaction that members of their cohort were receiving poor supervision. Some student participants reported providing suggestions for improvements in the supervisory experience, including the need for clear supervisory standards, oversight of supervisors, reduction of inconsistent communication from different supervisors, and lack support for students themselves (e.g. how to limit burnout, manage transference). Lastly, some 
respondents reported that overall, there was a lack of more intense supervision at the start of the

practicum they felt was needed due to the mismatch in training level and complex patient

population.

Well, supervision is just having a sounding board and being able to talk about your ideas and how you've understood things and how you might be able to understand them in different ways, getting ideas about resources to use or different interventions that they have found helpful in their clinical practice. Right down to just getting feedback about from your video. Like, oh, you should not write notes so much, you should lean, just like those kinds of nuts and bolts things have been really helpful. When you are learning you have a lot on your mind and you are just trying to remember how to do the intervention but there is a lot of other things that go into too, in terms of building rapport with your clients and making them comfortable and body language, and all of that. So that has been great from our supervisors. (Participant 12, Student)

And then individual supervision, and for me at the time, that was with [clinical supervisor]. And I liked [their] supervision and I also liked [them]. (Participant 7, Student)

So my experience is that it's kind of luck of the draw in terms of who you get as a supervisor. So, I was really fortunate in who my supervisors were, but I know other students did not have as good as a training experience that I had. And I find that to be pretty problematic and just kind of disappointing for those students who didn't have the same experiences. And I feel like there must be ways that that can be improved and I know my cohort anyways tried to make an effort to make, to inform people of the problems that were there and make some changes. And I'm not sure how well those have been implemented or how much those have been taken up by decision makers. (Participant 1, Student)

I think some supervisors were better than others and I don't think there was a standard for how supervision was being conducted and that bothered me...Y You know, in terms of what kinds of things do you, should you expect in supervision. Like, do supervisors listen to tapes before sessions, or not? How do the supervisors approach you? That kind of stuff. What kinds of things should you be preparing for supervision? So, having those kinds of expectations, I think laid out more clearly would have been excellent. (Participant 2, Student)

The only thing I thought about too was, you know, for students who do have experience in the mental health system as clients themselves, or are coping with mental health concerns, having a more, I don't know if it wasn't accepting, I just feel like it was, I feel like some of the supervisors weren't sure how to manage the students' needs at some points. And speaking for myself personally, and from other people I know who were there who had similar concerns, that you know, bringing up personal complaints and issues and needs that related to mental health in the context of treatment and training, it 
wasn't clear how, it wasn't that they were rejected, it was just it seemed like two isolated silos and it wasn't... clear how, you know, it's natural that these things are being triggered because you're in these situations and so on and so forth. And I think that kind of piece, just, you know, having a clear understanding of how to deal with students' needs. And, I think that's a general issue for individuals in mental health and the burnout there. Cause I was burning out at times there, and so I think addressing students' burn out and how to be supportive in their first practicum or whatever practicum they're in, I think it's something that is, was not really on the books. (Participant 2, Student)

I think it's just maybe coming up you now after being on several practicum interviews and hearing about sort of the stages of being introduced at those places. So, for example, watching somebody do three assessments and then being supervised in the room on three assessments before doing it on your own, whereas they kind of just were like, "start doing assessments now"... Which can be beneficial because, I mean you learn how to sort of think on your feet and think about this stuff yourself. But, I guess having that sort of intense supervision especially upfront would have been very helpful. (Participant 8 , Student)

Different supervision modalities. Participants reported that having access to different supervisors and supervision modalities was beneficial because this provided varying points of view, increased availability of supervisors, and allowed for structured (e.g. weekly scheduled meetings) and unstructured supervision (e.g. open door policy). Student participants spoke positively about their group supervisory experience, enjoyed reviewing cases with their cohort, and appreciated didactic training in a group format. They said they felt supported by and learned from their cohort, most of whom completed the practicum at the same time. Some respondents stated that they appreciated having $\mathrm{PhD}$ level peer supervisors and were disappointed that this element was no longer part of the program.

I think, so intra[professional], it benefits patients indirectly I guess because we are students, we are learning, we really don't have all of the answers all of the time, so to be able to do group supervision and individual supervision is very helpful because we get that kind of support from people who have a lot of experience providing these treatments. ... Doing group supervision gives us more exposure so we get to hear about cases we are not treating but we still get to hear about what they are doing and how they are addressing that, and what is working and what has been challenging, and kind of think about it as a group. So yeah, that is great. (Participant 12, Student) 
Yeah, because the director, I mean [the clinical supervisor] was always there or someone else was always there. And there was always, either talking to like, the same level cohort or senior graduate students who were around. There's always people to check in with and to have around. So I think that was very helpful. (Participant 2, Student)

I had a supervisor who is a faculty in Psychology, here and I could shoot her a quick email, and ask her if I, like, something aside from our scheduled supervision. And, also, I mean, all the students there are so supportive of each other. It's the best part of about that clinic, is the environment, and if you had a bad day, you can walk into someone else's office, and you can talk to them about it, and they would be open to that. So, that's a really important part of the clinic success. And then in terms of, I guess actual client care, I mean, people are always open to chatting, and throwing out ideas, and helping you brainstorm what to do. It's just, it's such an innate a part of the clinic. Like, it's just how it is. And it's how it always will be, I think, because that's what we billed it as. (Participant 6, Student)

Having your cohort member just across the hall is really, really great for social support. I really liked having people that were in the same position as me, that I didn't feel were superior to me or inferior to me to consult with or just to commiserate with, or to experience like joy and pride with and all of your successes with. Because we're all having the same senses of failure and success together. So, it's, and your, you know, doing your assignments at the same time as people, so they really, really identify with what you're going through. So having people, especially if you're new to Toronto, or new to the program, it's like really your core, and they're right there doing the same things you're doing. So, that's maybe, you know, the second most beneficial thing, after just the exposure. (Participant 4, Student)

So...each of the supervisors meets with the students weekly, for an hour. And then we do group supervision once a month... Or I, every other month, every other week, I'm sorry. And I think that those opportunities are really helpful. Like I couldn't imagine that not being as intensive it is, I think that those are really beneficial. So I think the intraprofessional, or supervision, I think it's nice that they have opportunities to meet with multiple providers. So, if I'm here, they can meet with me and even if I'm not providing them supervision, if they have a quick question and their supervisors not available. But also try to be, you know, not get too involved in be respectful of the supervision that's being provided. But if it's a situation that just needs instant communication. And then, what we did this year, was we switched supervisors midpracticum. So were going to look at that and see if that's a helpful model to expand options for, you know, for students, in learning and things like that. (Participant 10, Clinical Supervisor)

I guess one thing that was a challenge that might have been specific to my cohort was we initially had a peer supervision going on and then because of some issue that happened with one of students, that was no longer available to us. And most of us really appreciated that experience and were getting a lot out of that. And it was kind of stopped with no explanation and no ideas around if it would be coming back. And in terms of 
training later on in my time at Ryerson, I was looking forward to potentially being a peer supervisor, but it sounds like, or it looks like that's not an option anymore so that was also a drawback I think. That was a great thing that the clinic offered that it stopped offering. (Participant 1, Student)

\section{Discussion}

Interdisciplinary primary health care teams and access to psychotherapy have been identified as key elements to improving the mental health of Canadians (OMHLTC, 2011; MHCC, 2012a). Substantial reforms have been made to Ontario's primary health care system in the past decade with the establishment of hundreds of FHTs (OMHLTC, 2016). FHTs provide patients with a range of services from various health care disciplines working in collaboration, a move away from the traditional single practitioner model (Farmanova et al., 2013; Rosser et al., 2011; Slater, Nicholas, Leung, \& Lofters, 2016). Including psychologists in this mix can improve access to psychotherapy, a well-documented treatment for a range of mental disorders (Butler et al., 2006; Chambless \& Hollon, 1998; Chambless \& Ollendick, 2001; Health Care, 2002; Hofmann et al., 2012; Hunot et al., 2007; Hunsley, 2002; Hunsley et al., 2014; Myer \& Payne, 2006; Olatunji et al., 2012; UKDH, 2001). Emerging evidence indicates that psychotherapy provided in primary care settings is effective, though there is limited research specifically examining the employment of psychologists in Canadian FHTs (Chomienne at al., 2011; Cordeiro et al., 2015; Cuijpers et al., 2009; Kates et al., 2011; Linde et al., 2015; Seekles et al., 2013). Few psychologists work in primary care settings (Cordeiro et al., 2015; Moulding et al., 2009; Mulvale \& Bourgeaut, 2007) and training opportunities in the area are limited (Bray et al., 2003; Grenier et al., 2008; Masters et al., 2005; Twilling et al., 2000). The present study significantly adds to this growing field by exploring perspectives on interprofessionalism, patient care, and student training at the PTC, a treatment delivery and student training site integrated into St. Michael's Hospital's set of six FHTs. This mixed methods study examined perspectives 
from health care providers, clinical psychology graduate students, PTC clinical supervisors, and patients through an online study (Phase 1) and individual interviews (Phase 2).

\section{Perspectives on Interprofessional Patient Care and Student Training}

This two part study used a concurrent triangulation explanatory design. First, participants completed an online survey comprised of validated and newly generated questionnaires in order to balance generalizability with the refinement needed to evaluate the unique training and treatment delivery model at the PTC (Valentine et al., 2015). Data from these surveys generally indicated positive interprofessional beliefs and experiences, though results indicate there may be room for improvement. This was confirmed by individual interviews. Participants described overall positive experiences and beliefs related to collaborative patient care, interprofessionalism, and student training at the PTC, though there were of course individual differences. Conflicting perspectives were noted as well, highlighting tensions, competing demands, varying expectations, and general challenges related to establishing a new clinic. These tensions are not uncommon when merging traditional solo practitioners into a shared practice, which can lead to tension due to power dynamics and historical biases (Beales, Walji, Papoushek, \& Austin, 2011; Soklaridis et al., 2007). Participants described many of these challenges improving over time. This work adds to the small but growing research exploring interprofessionalism in primary care environments that provide psychotherapy, including Psychology (Chomienne at al., 2011; Cordeiro et al., 2015; Giordano et al., 2013).

Patient care. Overall, the PTC was considered a positive addition to the St. Michael's Hospital FHTs and participants were satisfied with its services. The PTC was reported to fill a gap in services at the FHTs and improved access to high quality, evidence-based psychological services that most patients would not otherwise have had access to due to financial constraints 
and long wait times in the community. Participants described the treatment of anxiety and depressive disorders as being particularly efficacious. Adding psychological services to the FHTs also appeared to legitimize these services and reduce the stigma of accessing them (Cordeiro et al., 2011). Services provided at the PTC were seen as complementary to other FHT services and facilitated referrals to internal FHT programs. The geographical location and the building were said to be accessible, though there were challenges with the elevator at times and for patients who lived across town. The most frequently reported challenge related to the PTC was the long waitlist. Although wait times may be shorter in some primary care settings, some Canadian FHTs reported higher referral rates than outpatient clinics because they are viewed as more accessible (Kates et al., 2011a). This may be due to what one health care provider described as greater trust in in-house services due to shared records and a greater awareness of the quality of service patients are receiving. Furthermore, dissatisfaction with mental health services prior to the opening of the PTC was noted, something that has been described in other primary care settings without mental health services on site (e.g. Vickers et. 2013).

Positive interprofessional perspectives and experiences. Compared to past research, participants reported moderate to high levels of interprofessional patient care and beliefs, achieving scores that range from 75 to $84 \%$ of the measures' total scores. Participants highly endorsed that interprofessionalism improved the quality of patient care, communication between different disciplines, decision making and treatment planning for patients, and overall collaborative patient care. In general, they appreciated the interprofessional team approach to patient care. These sentiments were echoed in interviews; the majority of participants spoke favourably about interprofessionalism and their experiences at the PTC and FHTs. Compared to more established health care providers, clinical psychology graduate students reported lower 
ratings for interprofessional experience and beliefs overall, but not all differences were statistically significant.

Results were similar to past research with some distinctions. To start, some interprofessional scores obtained were higher than past research (Goelen et al., 2006; Gotlib Conn et al., 2014; Hawk et al., 2002; Kenaszchuk et al., 2011, 2012; Robbens et al., 2012). Allied health care professionals and younger physicians may be more open to the team-based model compared more seasoned physicians or those in supervisory positions (Kenaszchuck et al., 2010; Gotleib Conn et al., 2012). This may account for the elevated scores at the St. Michael's FHTs where there is a large proportion of newer physicians and allied health care providers.

Alternatively, in some cases scores obtained by graduate students and health care providers were similar to initial baseline scores prior to the implementation of an interprofessional intervention or education (e.g. Fulmer et al., 2005; Giordano et al., 2013; Pinto et al., 2012). In interviews, graduate students reported receiving limited FHT and interprofessional specific training at the PTC, which may account for this finding. As well, the majority of health care providers were physicians, who have been found to have lower scores than nurses and other allied health professionals (Fulmer et al., 2005; Giordano et al., 2013; Kenaszchuk et al., 2011; Leipzig et al., 2002). On one survey, graduate students indicated greater isolation and less accommodation from other team members compared to health care providers. Health care providers, alternatively, reported more favourable interprofessional views, did not experience isolation, and felt accommodated by other team members. Similar group differences were found in past research that found physicians experienced less isolation and greater accommodation by team members than other allied health care professionals (Gotlib Conn et al., 2014; Kenaszchuk et al., 2012). 
Collocation. Collocation was highlighted as improving access to care, facilitating interprofessional patient care, and increasing referrals to collocated services. These findings were similar to past research at the same set of FHTs (Slater et al., 2016). Collocation of psychologists and physicians has been shown to increase interprofessional satisfaction and improve the quality of mental health care in primary care settings (Chomienne et al., 2011; Clatney et al., 2008; Cohen \& Peachey, 2014; Cordiero et al., 2015; Craven \& Bland, 2006; Farrar et al., 2001; Peachey et al., 2013; Swenson et al., 2008; Vickers et al., 2013; Xyrichis \& Lowton, 2008). However, the fact that certain disciplines are housed on different floors was identified as a barrier to face-to-face consultations, the preferred means of communication (Brown et al., 2009; Slater et al., 2016). Case in point, participants interviewed relayed that collaboration between Psychology and Medicine was greater when they were collocated on the same floor but appeared to reduce once Psychology moved downstairs.

Lack of team meetings. Collocation is not enough to promote interprofessional interactions; this is ideally done through increased contact and communication among team members (Beales, Walji, Papoushek, \& Austin, 2011; Sargeant, Loney, \& Murphy, 2008; Wener $\&$ Woodgate, 2016). Teams who meet regularly typically have greater communication and interaction than those without regular meetings (Mulvale et al., 2008). Thus, it is important to carve out time and space for various disciplines to build relationships and communicate regularly, which may cultivate greater understanding and respect among professionals (Goldman et al., 2010a; Sargeant et al., 2008). In interviews, participants recommended more frequent interprofessional team meetings to improve communication and provide more collaborative patient care. The lack of designated time to interact with and learn from other team members has been reported in other FHT settings due in part to physician fee structures that may financially 
penalize physicians from attending such meetings (Beales et al., 2011). This supports responses from several interviewees who made reference to the fact that team meetings were infrequent due to a lack of availability or willingness from physicians.

Respect. Participants interviewed generally reported a culture of respect, openness, and equality at the PTC and FHTs, though some instances of conflict and lack of respect were identified. This is potentially due to the lack of time and space needed to develop new relationships and challenge historical biases (Goldman et al., 2010a; Sargeant et al., 2008). Survey data supported this generally positive though mixed experience of respect from team members. For example, some respondents felt their work was considered less important or lower status by other disciplines. It was also reported that not all team members appeared open to new interprofessional practices or putting in effort to learn about the scopes of practice of other disciplines, something identified in other FHT settings (Goldman, Meuser, Lawrie, Rogers, \& Reeves, 2010b). However, participants overall reported that their time working at the FHTs led to a greater understanding of the scopes of practice of other professionals with whom some had not previously worked and experienced a limited hierarchy.

Limited hierarchy and scope of practice. Although some participants experienced a hierarchy with the physicians on top, others reported experiencing no hierarchy or a limited one. As one participant pointed out, some hierarchy may be beneficial and this is supported by research indicating the value in having clear leadership and management (Goldman et al., 2010a). Strong physician leadership can also promote the interprofessional model for physicians less amenable to adopting interprofessional practices (Gocan, Laplante, \& Woodend, 2014; Goldman et al., 2010a; Goldman et al., 2010b). It is also recommended that FHTs have clearly defined roles and expectations among team members in order to promote team cohesiveness 
(Gocan et al., 2014; Goldman et al., 2010a; Grumbach \& Bodenheimer, 2004; Xyrichis \& Lowton, 2008). Participants reported that professional roles and responsibilities were initially not well-defined, though became more established over time. However, some noted that there some overlapping scopes of practice remained which resulted in duplication of services or conflicting treatment approaches. Overlapping roles and unclear responsibilities can potentially lead to conflict among team members (Brown et al., 2011). While some conflict between colleagues was reported, participants generally described positive collaboration in order to resolve issues and carve out more defined roles. For example, one health care provider explained not previously understanding what psychologists did but came to appreciate their skill in diagnostic clarification and realized their own shortcomings in this area. This flexibility and collaboration in defining roles can allow health care professionals to adapt to the changing needs of patients and the interprofessional team (Gocan et al., 2014).

Interprofessionalism collaboration and communication. Level of interprofessionalism appeared to be vary based on participants' level of training, discipline, level of leadership, and individual characteristics. For example, strong relationships were reported between Psychology and Social Work, but weaker with Psychiatry. For effective collaboration, it is important to develop specific organizational structures and procedures to promote interprofessional team functioning (Beales et al., 2011; Wener \& Woodgate, 2016). Participants described a lack of clear interprofessional framework at the PTC and FHTs. In fact, some graduate students were unaware of the FHT team-based model. Interprofessional teamwork at the FHTs was generally described as occurring on a case by case basis, often with different health care providers working separately with limited communication or joint treatment planning. Similar experiences at other FHTs have been documented. One study reported that FHT staff described positive 
interprofessional collaboration when it occurred, but noted that it was not a common occurrence (Beales et al., 2011). This matches survey responses that indicated that perceived need for interprofessional cooperation was not matched by participants' lived experience.

Interprofessional collaboration may be impacted by staff and students' part-time schedules, as well as the high turnover of students as they move through their training, which can reduce the frequency of collaboration and communication (Beales et al., 2011). Graduate students echoed this in the interviews noting that their full practicum schedule did not allow for additional time to attend interprofessional activities that were available.

Overall, communication was described as limited in nature, particularly in person communication. Lack of time was cited as the biggest barrier to more integrated care and communication. To work around this issue, EMR use was reported as an efficient way to initiate and maintain interprofessional contact. EMR use has been identified as a means to improve communication and facilitate patient care (Gocan et al., 2014; Goldman et al., 2010a; Mulvale et al., 2008; Ragaz, Berk, Ford, \& Morgan, 2010). However, some participants felt there was an over-reliance on this method and suggested increased team meetings as a way to promote greater interprofessionalism. Nonetheless, participants noted that most team members had an open door policy or attempted to be available for consultation if requested.

Consultation. When interviewed, some graduate students reflected that consultation was not always bi-directional. For example, some students recalled times when they reached out to physicians, but physicians did not in turn reach out for students' expertise. This is in contrast to survey results that indicate health care providers received and provided significantly more consultation than clinical psychology graduate students. In fact, one-third of students indicated receiving no consultation and more than half did not provide any consultation to other team 
members.

The majority of consultations graduate students did engage in occurred with Social Work, physicians, Psychology, and Psychiatry, all of whom have expertise in mental health. Students reported two key reasons why Social Work was so readily accessed for consultation: 1) it was located on the same floor; and 2) there was positive modeling of interprofessional collaboration between PTC clinical supervisors and Social Work. Health care providers, on the other hand, reported that most consultation occurred with the pharmacist, physicians, nurses, and nurse practitioners, disciplines that have more traditionally worked together. Both participant groups reported lower rates of consultation with Dentistry, Chiropractic, and Dietetics, disciplines that have not historically been integrated into primary care settings. These consultation rates match results from a recently published study from the same set of FHTs (Slater, Nicholas, Leung, \& Lofters, 2016). It is possible that low levels of consultation are related to students' low confidence in contacting their more seasoned team members.

Graduate student confidence in interprofessional skills. In general, graduate student participants reported moderate to high confidence in their interprofessional self-efficacy as a result of their training. Scores were similar to though slightly lower than past research, though it varied by the study (Mann et al., 2012; Oza et al., 2015; Vari et al., 2013). It should be noted that scores were significantly higher for student participants in their upper years of graduate training. This is not surprising given that upper year students had additional training at the time of data collection due to progress in their graduate program.

Overall, graduate students reported confidence working as a member of an interprofessional team (i.e. forming a team, resolving problems as a team, creating collaborative care plans, and interacting and communicating with other professionals) and understanding team 
learning objectives. Participants felt less confident in their ability to provide feedback to professionals or patients about interprofessional team functioning or their ability to evaluate the interprofessional FHT model, which is similar to past research (Mann et al., 2012). This may account for students' low level of consultation and matches opinions expressed in Phase 2 for which participants felt less confidence interacting with more established health care providers. Low confidence has previously been reported to negatively impact students' ability to interact with seasoned professionals, though once students bridge this gap, it appears that their confidence increases (Dornan, Boshuzen, King, \& Scherpbier, 2007). Interprofessional training may also improve confidence, though outcomes vary by discipline (Vari et al., 2013). Student expressed interest in greater interprofessional training, which has been recommended to support the growing field of interdisciplinary primary care psychology (Garcia-Shelton \& Vogel, 2002; Linden et al., 2005; Pingitore, 1999; Nash et al., 2012; Schulte et al., 2004; Sladen, 1979). Interprofessional primary health care training is particularly emphasized for students early in their training, such as those training at the PTC (Talen et al., 2005).

Student training at the PTC. Overall, the PTC was described as a unique and positive training experience. PTC supervisors and graduate students reported that they appreciated the interprofessional training environment, working with a diverse patient population, developed a strong foundational clinical skill set that complemented classroom learning, and had access to a range of training useful resources on site (e.g. library, assessment measures, audio-visual recording for supervision). The fact that students in the same cohort completed the placement at the same time was noted as being particularly beneficial because they were able to support and learn from one another.

The experiential learning environment allowed graduate students to gain a better 
understanding of FHTs and interprofessionalism. Experiential learning can improve students' understanding of other team members' scope of practice, as well as gain competence and capacity to work in primary health care settings (McDaniel, Belar, Schroeder, Hargrove, \& Freeman, 2002; Twilling et al., 2000). Student participants described feeling prepared for their next clinical practicum or internship and felt their training gave them an edge over other students. However, a lack of specific interprofessionalism and FHT training was reported that some graduate student participants found disappointing. Throughout the interviews, student participants conveyed a lack of knowledge about services at the St. Michael's Hospital FHTs generally, the FHT model, or elements that promote interprofessionalism, though this varied by participant and some were more knowledgeable than others. As well, the majority of participants had difficulties identifying services beyond what was provided at the PTC or the FHT site where the PTC is located. This lack of awareness of all available FHT resources was also identified in a recent study of FHT health care providers (Slater et al., 2016).

Supervision. Supervisors were lauded for their availability, as well as their positive role modeling and encouragement of interprofessional collaboration. Positive role modeling is key for interprofessional training (McDaniel et al., 2002). However, there were some who noted that the competing demands of the supervisors at times interfered with optimal training. Participants reported that the quality of supervision was dependent on the supervisor and that quality contributed to whether or not students had a positive training experience. Supervision quality has a substantial impact on students' ability to learn (Ladany, Mori, \& Mehr, 2013). Many students reported exemplary supervisory experiences and felt supported. Others described poor supervision for which supervisors checked email during supervision, were unavailable when needed, were disorganized or lacked structure in supervision, and did not provide enough 
oversight. These latter experiences may be considered minimally adequate or inadequate supervision, which one study estimates over $90 \%$ of student trainees experience at some point in their training (Ellis et al., 2014). Some supervision can go beyond being inadequate and can even have lasting harmful effects leading supervisees to feel hurt, confused, and distressed (Ellis, 2001; Ladany et al., 2013; Nelson \& Fredlander, 2001). Participants suggested ways to improve the supervisory experience, including having more clear guidelines as well as increased supervision at the start of the placement particularly due to the complex patient population. Guidelines such as the Ontario Psychological Association's (OPA) Bill of Rights and supervision contracts can guard against negative experiences by creating clear expectations (Bernard \& Goodyear, 2014; Ellis et al., 2014; OPA Working Group on Clinical Supervision, 2015). As well, graduate students reported that some of these challenges were minimized by the presence of different supervisory modalities (e.g. group supervision, peer supervision, $\mathrm{PhD}$ level peer supervisors) and availability of unstructured supervision (e.g. open door policy of clinical supervisors).

\section{Limitations and Future Directions}

The foremost limitation is sample size. Future research would benefit from greater participation from each of the participant groups included in this study. Although both phases included representatives from each intended participant group, there were difficulties recruiting patients and health care providers. There is some literature to suggest that physicians, for example, are difficult to recruit due to time constraints and may benefit from a range of resources to improve participation rates such as personalized and frequent contact, which was not available due to limited resources (Asch, Connor, Hamilton, \& Fox, 2000; Parkinson et al., 2015). Previous affiliation with the researchers and the PTC may, however, have been a factor in the 
higher response rates for clinical psychology graduate students. Future research may benefit from exploring additional recruitment options and incentives to increase the participation of health care providers. It may also be helpful to explore differences among different disciplines, which was not explored in this study due to limited variability among health care professionals' disciplines.

Resources were similarly unavailable to more actively engage patient involvement, which can increase participation rates but is often considered onerous (Callard et al., 2014). Flyers may simply not have been enough to attract attention to the evaluation project and it is unclear whether health care providers actually referred patients to the study. There is also some evidence that primary care providers may be hesitant to refer patients experiencing mental illness to engage in research for fear of interfering with the patient-provider relationship, overburdening a vulnerable population, or perhaps more simply due to the need to prioritize other appointment needs over research engagement (Callard et al., 2014; Mason et al., 2007). Moreover, patients experiencing socioeconomic difficulties, such as those at the PTC, may not trust research generally, have transportation difficulties, lack access to the internet, or fear the stigma associated with mental health issues, all of which may detract from research participation (Woodall, Morgan, Sloan, \& Howard, 2010). It will be important to actively elicit patient engagement in future research to ensure that their perspectives are represented. Lastly, it would be beneficial moving forward to explore whether or not the implementation of an interprofessional intervention leads to greater interprofessional beliefs and practices, especially for graduate students.

\section{Implications and Conclusions}

The addition of the PTC to St. Michael's FHTs was identified as positive and increased 
access to evidence-based psychotherapy. Similar to past research, participants appreciated and were satisfied with the services provided at the PTC (Chomienne et al., 2011; Cordeino et al., 2015). Overall, health care providers and clinical psychology graduate students reported beliefs congruent with the interprofessional FHT model, valued working collaboratively, and experienced a limited hierarchy overall. However, participants identified a number of barriers to fully embracing the team approach, such limited team meetings, limited time, lack of in person communication, and the fact that not all professions were located on the same floor. Students also reported wanting more specific training in the area. Nonetheless, participants reported generally having positive, respectful interactions with team members, frequently used EMR communication, and collaborated on a case-by-case basis.

Training opportunities in interdisciplinary primary care settings are limited (Bray et al., 2003; Grenier et al., 2008; Masters et al., 2005; Soklaridis et al., 2007; Twilling et al., 2000). Graduate students reported benefitting from the training at the PTC, which potentially gives them an edge by providing them experience in the emerging field of primary care psychology (GarciaShelton \& Vogel, 2002; Linden et al., 2005; Pingitore, 1999; Nash et al., 2012; Schulte et al., 2004; Sladen, 1979). Indeed, students described feeling well-prepared for future training experiences and appreciated the interprofessional skills they gained through experiential learning. 


\section{General Discussion}

Despite high rates of mental illness in Canada and the high economic impact, there is limited access to psychotherapy (Cohen \& Peachey, 2014: Lesage et al., 2006; Lim et al., 2008; MHCC, 2012a, 2012b; Smetanin et al., 2011), an effective treatment for a range of mental disorders (Butler et al., 2006; Chambless \& Hollon, 1998; Chambless \& Ollendick, 2001; Health Care, 2002; Hofmann et al., 2012; Hunot et al., 2007; Hunsley, 2002; Hunsley et al., 2014; Myer \& Payne, 2006; Olatunji et al., 2012; UKDH, 2001). In the past decade, the Ontario government has transformed the delivery of primary care with the creation of over 200 interdisciplinary FHTs, a model that may improve access to mental health care, especially if access to psychotherapy is included (Gagné et al., 2006; Kates, 2008; Kates et al., 2011; MHCC, 2012a; Mulvale et al., 2008; OMHLTC, 2011, 2016; Rosser et al., 2011). In fact, this model is proving successful in Australia and the UK where psychotherapy is being recommended as a first-line treatment (Fletcher et al., 2009; Moulding et al., 2009; UKDH, 2001). However, there is limited research on the impact of including psychologists and psychological services in FHTs (e.g. Collier, 2011; Cordeiro et al., 2015). Moreover, there are few training opportunities for clinical psychology graduate students to gain experience in this growing field (Bray et al., 2003; Grenier et al., 2008; Masters et al., 2005; Twilling et al., 2000).

Two studies were conducted to explore the impact of integrating a psychology training clinic into a set of FHTs at St. Michael's Hospital. The findings represent a significant contribution to the FHT and primary care psychology literature by documenting the benefits and effectiveness of adding psychological services to an FHT setting, all while showcasing a positive and valued training experience for clinical psychology graduate students. 


\section{Individual CBT Provided in a FHT and Student Training Clinic}

An ongoing challenge of providing psychotherapy that is covered by Medicare is doing so in a timely fashion. The current study found that participants were on the PTC waitlist between two and a half months to close to one year, with an average duration of six months. This wait is similar to or shorter than other publically funded mental health services; however, it may be higher than other primary care settings (Anderssen, 2015; Cordeiro et al., 2015).

Duration ranged from four to 16 sessions with participants who completed treatment receiving an average of 12 sessions, which is similar to though somewhat greater than other primary care sessions (Cape et al., 2010; Cordeiro et al., 2015; Cuijpers et al., 2009; Linde et al., 2015; Seekles et al., 2013). This suggests that from a planning perspective, waitlist and treatment duration at the PTC are consistent with other similar primary care settings. It is important to note that participants' symptoms did not significantly change while on the waitlist.

With respect to treatment effectiveness, individual CBT provided by clinical psychology graduate students to patients of the FHT was found to lead to significant improvements in symptoms of anxiety and depression despite the fact that clients had complex diagnostic presentations. Eighty percent of participants either no longer met criteria for their primary and sometimes secondary diagnoses, or were in partial remission. Twenty percent experienced no diagnostic change. Symptoms of overall mental health as measured by the OQ-45 reduced significantly from pre to post treatment. Moreover, the shift of scores from above to below the clinical cut-off was also statistically significant. These findings were similar to and in some cases greater than past research using the OQ-45 (e.g. higher rates of reliable change, larger effect size) (Chommienne et al., 2011; Cigrang et al., 2006; Lambert et al., 2002; Nyman, Nafziger, \& Smith, 2010). Participants also experienced a statistically significant decrease on 
the DASS-21 throughout treatment, as well as a significant reduction in depressive symptom severity from pre to post. However, there was not a statistically significant decline in symptom severity for anxiety and stress symptoms. In fact, pre-post changes were similar to, though in some cases lower than past research, which may be accounted for by the limited change in the latter two factors (Bradbury et al., 2008; Dear et al., 2011; Titov et al., 2010; Troeung et al., 2014). Medication use, working alliance, and social support could not predict these changes, though caution is needed in interpreting these finding due to the small sample size and related low power. Participants reported a high level of satisfaction with the services at the PTC and strong working alliance with their graduate student psychotherapists.

\section{Perspectives on Patient Care, Interprofessionalism, and Student Training}

Overall, participants indicated positive interprofessional beliefs and experiences, though some challenges and mixed feedback were reported. The PTC was identified as a positive addition to the St. Michael's Hospital FHTs that complemented other FHT services and was well-received and valued by health care providers and patients. The main drawback voiced was the long waitlist, which may be due to higher referral rates seen in some primary care settings because they may be viewed as more accessible (Kates et al., 2011a).

The collocation of services reportedly increased participants' trust in the services, improved access to psychotherapy, increased referrals, and may have reduced the stigma of accessing mental health services. This supports past research that suggests collocation of psychologists and physicians may improve collaboration between the professions, as well as the quality of mental health care in primary care (Chomienne et al., 2011; Clatney et al., 2008;

Cohen \& Peachey, 2014; Cordiero et al., 2015; Craven \& Bland, 2006; Farrar et al., 2001; Peachey et al., 2013; Swenson et al., 2008; Vickers et al., 2013; Xyrichis \& Lowton, 2008). 
However, the fact that certain disciplines are housed on different floors was identified as a barrier to face-to-face consultations, the preferred means of communication (Brown et al., 2009; Slater et al., 2016). In fact, participants recommended more regular team meetings, which can improve interprofessional communication and collaboration by affording time and space to build relationships among team members (Goldman et al., 2010a; Mulvale et al., 2008; Sargeant et al., 2008). More frequent contact may also challenge historical biases and engender greater understanding and respect for different disciplines (Beales et al., 2011; Sargeant et al., 2008; Wener \& Woodgate, 2016). Tensions between disciplines are not uncommon when forming new interprofessional teams (Beales et al., 2011; Soklaridis et al., 2007). Although respondents typically reported positive interprofessional interactions and experienced a culture of respect, some reported mixed experiences due in part to lingering power dynamics.

Interprofessionalism appeared to be vary by profession and person, with some describing more comfort with the model than others. Respondents typically reported positive interprofessional beliefs that were similar to past research, though scores on some measures were slightly higher than others (e.g. Goelen et al., 2006; Gotlib Conn et al., 2014; Hawk et al., 2002; Kenaszchuk et al., 2011, 2012; Robbens et al., 2012). These differences may be due to the large portion of allied health care professionals and younger physicians who typically have more favourable views of team-based care (Kenaszchuck et al., 2010; Gotleib Conn et al., 2012). Others scores were slightly lower and in some cases more similar to baseline scores prior to a training intervention (Fulmer et al., 2005; Giordano et al., 2013; Pinto et al., 2012). This may be due to limited specific interprofessional training for clinical psychology graduate students. While students reported high confidence in a range of interprofessional domains, lower confidence was reported for providing feedback to other professionals or evaluating the FHT 
model. They also indicated low levels of consultation with their team members. Responses from interviews indicate this may be because students felt less confident interacting with more established health care providers. Low confidence can negatively impact students' ability to interact with seasoned professionals, though interprofessional training may improve their confidence in this skill (Dornan et al., 2007; Vari et al., 2013).

Participants reported experiencing a limited hierarchy, though some experienced one with physicians on top. However, some hierarchy may be beneficial for leadership and accountability purposes (Goldman et al., 2010a). Scopes of practices at the FHTs and PTC were noted as evolving over time. This kind of flexibility may be beneficial to adapt to changing needs of patients and the team (Gocan et al., 2014). Collaboration was said to be on a case by case basis rather than regularly occurring, which has similarly been documented in other FHT settings (Beales et al., 2011). Reasons for this may include part-time schedules and high turnover of students as they complete their practical training (Beales et al., 2011). Interprofessional communication was also described as limited in nature with lack of time and different work schedules as key barriers to engagement. The EMR was identified as a way to work around these barriers (Gocan et al., 2014; Goldman et al., 2010a; Mulvale et al., 2008; Ragaz et al., 2010). However, some participants were concerned that the EMR was relied upon too heavily for interprofessional communication. With such limited research examining curriculum for psychologists in interprofessional settings, this remains an important area to explore.

The PTC was seen as a strong and unique training experience that provided clinical psychology graduate students with a solid foundation and experiential training in the interprofessional FHT model. Students appreciated working with different disciplines and the diverse patient population. The lack of specific interprofessional training was identified as 
problematic and was exemplified in students' lack of knowledge, including limited awareness of services at other FHT sites. However, a study of the same FHTs with a larger sample of health care providers found they similarly did not have a great awareness of services provided at FHTs beyond their own site (Slater et al., 2016). Supervision, a key influence on students' training experience and learning (Ladany et al., 2013), was reported to be mixed with some students describing exemplary supervision while others described minimally adequate or inadequate supervision. This is in fact consistent with other training research which has found that upwards of $90 \%$ of student trainees experiencing inadequate supervision at some point in their training (Ellis et al., 2014). Implementation of clear guidelines such as supervisory contracts or guidelines such as the OPA Bill of Rights can guard against such negative experiences (Bernard \& Goodyear, 2014; Ellis et al., 2014; OPA Working Group on Clinical Supervision, 2015). Graduate students reported a number of positive supervisory experiences, including different supervisory modalities (e.g. group supervision, peer supervision, $\mathrm{PhD}$ level peer supervisors) and unstructured supervision (e.g. open door policy of clinical supervisors). Lessons learned for other primary care psychology training facilities include the need for explicit interprofessional, primary care training, ensuring consistent high quality supervision, and employing multiple levels of supervision.

\section{Strengths and Limitations}

Research, evaluations, and implementing new standard procedures in real-world settings are often difficult due to time constraints, lack of resources, and competing demands (Damschroder et al., 2009; Flottorp et al., 2003; Manca et al., 2014; Richter-Sundberg et al., 2015). However, it is important to know how evidence-based treatments perform in real world situations beyond the control exacted in randomized control trials, including testing interventions 
with complex patient populations (Kazdin, 2008). A key purpose of these studies was just that, to examine the effectiveness of individual $\mathrm{CBT}$ in a real world clinic, one that could potentially be replicated in other FHT and primary health care settings.

The greatest strength of this evaluation was the multifaceted way in which the services and the PTC were examined through three separate but interconnected studies carried out over the same time period. The mixed methodology provides a more solid understanding of the impact of the PTC and its services on patients, health care providers, and clinical psychology graduate students. The inclusion of multiple perspectives was another strength of this study. However, it also highlights the main weakness of these studies, the small sample size.

Although the sample size of Study 1 satisfied the requirements for the pilot study, statistical analyses, and produced large effect sizes, power would have increased with a greater sample size. A comparison group was deemed not-feasible due to challenges recruiting participants to attend symptom monitoring appointments at the PTC due to patients' lack of availability and a lack of resources to ensure a researcher was onsite to foster patient engagement (Callard et al., 2014). These issues similarly impacted Study 2 and patient involvement was even lower. It is possible that graduate students and primary care physicians were hesitant to refer patients experiencing mental health problems to the evaluation for overburdening them, prioritizing other issues, or fear of negatively impacting the patient-provider relationship (Callard et al., 2014; Mason et al., 2007). This may have been the case, as some study measures were not completed as planned by student clinicians (e.g., working alliance inventory), which may be additional evidence of this perceived burden. A lack of trust in research generally, transportation difficulties, lack access to the internet, or stigma associated with accessing mental health services may have also detracted from participation (Woodall et al., 2010). There were also difficulties 
recruiting health care providers, particularly for Study 2 Phase 2. This may be due to busy schedules, lack of sufficient incentives, and limited previous contact with the author (Asch et al., 2000; Parkinson et al., 2015). It could also be taken as a sign of limited previous experience working interprofessionally with Psychology, as well as a fear reporting the limited interprofessional contact. Similarly, previous affiliation may have been a factor in the high response rates for psychology students.

There were numerous logistical and practical challenges evaluating a vibrant and busy clinical environment. As the PTC and St. Michael's Hospital became more established, there were a range of changes. For example, there were staff changes, multiple revisions to the referral process for mental health services, opening of a new FHT clinic, new graduate student cohorts each year, and even new editions of the DSM and SCID. This presented a number of difficulties ranging from time delays (e.g. waiting for the availability of the SCID-5-CV) to adapting the evaluation (e.g. individual interviews versus focus groups as initially proposed). This flexibility was necessary in order to collect clinically useful data, as well as problem solve challenges as they arose (Kirchner et al., 2010).

Finally, there are a number of differences between the PTC and FHTs at St. Michael's Hospital and other FHTs across Ontario. First of all, to the author's knowledge, this primary care mental health care delivery and student training model is the first of its kind in Canada. Second, there are six sites associated with the St. Michael's Hospital's FHTs, providing care to tens of thousands of patients. Therefore, although these results may provide valuable information on the effectiveness of CBT in FHT settings, interprofessional patient care, and student training in an FHT setting, the results may not be generalizable to other primary care and student training settings. 


\section{Future Directions}

To build on this research, it will be important to replicate these findings in other FHT settings. In particular, it will be helpful to include a large sample size, employ a comparison group, and follow participants beyond the end of treatment. It may be especially valuable to conduct a multisite study of the inclusion of Psychology in FHTs. Other areas of focus could include further exploration of wait times as well as a cost-benefit analysis of providing psychotherapy in FHTs. Student training in primary care is another area that warrants further examination, including changes in perspectives of interprofessionalism before and after specific interprofessional and FHT training and the impact of supervision quality on student training. It may be helpful to investigate differences in interprofessional experiences and beliefs between disciplines including Psychology. Lastly, it will be important for future research to have greater patient engagement to ensure that the patient experience is well-captured.

\section{Implications and Conclusions}

In an article in the Globe and Mail, Anderssen (2015) argues passionately and comprehensively that psychotherapy should be a front-line treatment for mental illness in Canada, especially for depressive and anxiety disorders. In the UK and Australia psychotherapy is considered a frontline treatment even before pharmacotherapy, which is publically funded and therefore more accessible than it is in Canada (Anderssen, 2015; Fletcher et al., 2009; Moulding et al., 2009; UKDH, 2001). Yet, psychotherapy is out of reach for many Canadians due to the lack of support in the Canadian Medicare system and the high costs to access it in private practice (Hunsley, 2002; MHCC, 2012a; Myer \& Payne, 2006; Vasiliadis et al., 2009).

Instead, the majority of Canadians seek help from their primary care physician, who consequently may be the only mental health provider they will see for mental health care 
(Fournier et al., 1997; Health Canada, 2002a; Lesage, et al., 2006; Vasiliadis et al., 2009). Truth be told, primary care physicians, nurses, psychiatrists, social workers, and other allied health care professionals traditionally funded through Medicare have been holding the Canadian mental health care system together with wire and string for decades. All things considered, they have been doing an admirable job. Physicians see high rates of individuals with mental illness representing the most frequently billed category in primary care (Health Canada, 2002a). In fact, for decades, upwards of 75 to $80 \%$ of physician appointments focused on a mental health component (Gunn \& Blout, 2009; Sharp \& Morrell, 1989; Simon, 1992; White 2008). Physicians primarily provide pharmacotherapy for mental disorders, a finding supported in this study as well as others (Roberge et al., 2011, 2014). They also often provide psychotherapy, though many feel their training in the area is inadequate, are unclear on what psychotherapy is, and are dissatisfied with what they can offer their patients in the way of mental health treatment (Grenier et al., 2008; Hameed, 2015; Moulding et al., 2009). One reason physicians may overrely on medication or try their hand at psychotherapy is because they know that the majority of mental health services, particularly psychotherapy, are inaccessible to most Canadians; medication and physician provided care is the only reliable option (Anderssen, 2015). This point was echoed in this study by one health care provider who expressed gratitude for the PTC because it meant patients could now be offered a real choice of either medication or psychotherapy.

Physicians and psychologists have both expressed a desire for greater access to psychological services (Cohen \& Peachey, 2014; Kainz, 2002; Mulvale, 2006; Vickers et al., 2013). The inclusion of psychologists in primary health care has been shown to increase interprofessional satisfaction and the quality of mental health care in these settings (Chomienne 
et al., 2011; Clatney et al., 2008; Cohen \& Peachey, 2014; Cordiero et al., 2015; Craven \& Bland, 2006; Farrar et al., 2001; Peachey et al., 2013; Swenson et al., 2008; Vickers et al., 2013; Xyrichis \& Lowton, 2008). These studies similarly found that health care providers, graduate students, and patients were satisfied with the services provided at the PTC, saw it as filling a service gap, and increased access to high quality psychological services. Graduate students delivered effective individual CBT while gaining valuable skills working with a diverse and complex patient population. Patients with anxiety and depression particularly benefitted from these services, two of the most common mental disorders seen in primary care settings (Bland et al., 1997; Bray et al., 2003; White, 2008). The addition of the PTC allowed for patients to receive the recommended number of sessions on average, something not always feasible in primary care settings that tend to employ briefer models of interventions (Cape et al., 2010; Nieuwsma et al., 2012; Roy-Byrne et al., 2010). Moreover, participants reported that the collocation of services increased awareness of the role of Psychology and reduced the stigma associated to accessing psychotherapy. In fact, the greatest drawback reported with regards to the PTC was the long waitlist, which speaks to the confidence health care providers have in referring their patients there, as well as the high demand for psychotherapy seen in other FHT settings (Chomienne et al., 2011; Cordeiro et al., 2015; Kates et al., 2011a).

Thus, the inclusion of Psychology and student trainees into a FHT appears to be a successful, viable option to improve access to effective mental health services. The novel treatment delivery and student training model at the Ryerson University PTC and St. Michael's FHTs appears to improve access to mental health services, can likely be replicated by other FHTs, and may either reduce costs or be cost-neutral meaning that costs are offset by the health and economic benefits gained (Chrisholm et al., 2016; Dobson et al., 2008; Hunsley, 2002; 
Hunsley et al., 2014; Mulvale et al., 2008; Myer \& Payne, 2006; Roberge et al., 2004; Swenson et al., 2008). In fact, St. Michael's Hospital FHTs, including the PTC, have been acknowledged nationally (Health Care Innovation Working Group, 2012) and now internationally by the World Health Organization as a successful, innovative primary care model (World Health Organization, 2016).

Mental health strategies have outlined the need for greater access to psychotherapy through interprofessional primary health care teams (MHCC, 2012a; OMHLTC, 2011). Building partnerships between pre-existing FHTs, hospitals, post-secondary institutions, or professionals such as between Ryerson University and St. Michael's Hospital can streamline resources while meeting the unique needs of a given community (Kates et al., 2011b). St. Michael's Hospital is not alone in making innovative changes to the primary health care model to improve access to psychotherapy. FHTs in Hamilton have substantially increased access to psychotherapy (Kates et al., 2011a). The Ottawa SHARE program is another example of successful partnership and the inclusion of Psychology (Swenson et al., 2008). Yet, few psychologists work in primary health care and those that do typically work part-time (Cordeiro et al., 2015; Moulding et al., 2009; Mulvale \& Bourgeaut, 2007).

These studies are a part of a small but growing body of evidence that demonstrates how including Psychology into primary care can improve mental health care (e.g. Chomienne et al., 2011: Cordeiro et al., 2015). The addition of a fully licensed clinical psychologist can allow for a wide array of services for a range of ages. For example, a recent study examining services at a FHT that employs one full-time found that patients from across the lifespan achieved significant improvements for a range of issues, as well as high satisfaction ratings (Cordeiro et al., 2015). Results from this study showed similarly high levels of satisfaction with the PTC's services. 
There are hundreds of psychologists ready, trained, and licensed to administer psychological assessments and psychotherapy, as well as conduct research and program evaluations, who can be integrated into pre-existing or evolving FHTs should governments and policy makers decide now is the time to act (Cohen \& Peachey, 2014). 


\title{
Appendix A \\ Informed Consent for Study 1
}

Letter of Information and Consent to Participate in a Research Study

\author{
Study Title: The Effectiveness of Psychotherapy Provided by Graduate Students at an \\ Interdisciplinary, Primary Care Clinic
}

\section{Study Team:}

Principal Investigator:

\author{
Dr. Kelly McShane, C.Psych. \\ Ryerson University \\ 416-979-5000 Ext. 2051 \\ kmcshane@psych.ryerson.ca
}

\section{Co-Investigators:}

\author{
Ms. Jen Rouse, M.A. \\ Ryerson University \\ 416-979-5000 Ext. 2187 \\ jrouse@psych.ryerson.ca \\ candice.monson@psych.ryerson.ca
Ryerson University
416-979-5000 Ext. 6631
dbrecher@ryerson.ca \\ Dr. Diana Brecher, C.Psych.
}
Dr. Candice Monson, C.Psych.
Ryerson University
416-979-5000 Ext. 6209

\section{Introduction:}

Before agreeing to take part in this research study, it is important that you read the information in this research consent form. It includes details we think you need to know in order to decide if you wish to take part in the study. If you have any questions, ask a study doctor or study staff. You should not sign this form until you are sure you understand the information. All research is voluntary. You may also wish to discuss the study with your family doctor, a family member or close friend. If you decide to take part in the study, it is important that you are as accurate as possible about your health history and any medications you are taking. This will help prevent unnecessary harm to you. Before you decide whether you will participate, it is important for you to understand why the research study is being done and what it will involve. If there is anything in this consent form that is not clear to you or that you need more information on, please ask your study doctor.

\section{Background:}

The Psychology Training Clinic's (PTC) is the result of an emergent partnership between St. Michael's Department of Family and Community Medicine and Ryerson University. The PTC services are provided by Ryerson University graduate Clinical Psychology students (psychology interns) who are supervised by practiced clinicians registered with The College of Psychologists of Ontario.

The training clinic is committed to evidence-based practice, interprofessional team-based service delivery and training, and the use of technology to best serve patients and promote learning.

The purpose of this study is to determine if individuals experience greater psychological and physical symptom reduction and improved functioning as a result of receiving individual cognitive behavioural therapy (CBT) as compared to time spent on the waitlist for CBT. 
There will be approximately 25-50 participants recruited for this study from St. Michael's Hospital PTC who engage in standard practices at the PTC, including psychological assessment, placement on the waitlist, and undergoing individual CBT

\section{Description of the Research:}

You are being asked to consider participating in this research because you are a patient of the PTC at St. Michael's.

As part of your routine care, you would complete the following assessments and procedures at intake and/or before, during, and after treatment (CBT):

- Structured Clinical Interview for DSM-IV-TR (SCID): A comprehensive semi-structured interview administered by a supervised psychology intern that is approximately two hours in duration.

- Outcome Questionnaire 45.2 (OQ-45): A 45-item self-report questionnaire focusing on overall mental health-related symptoms.

- Depression Anxiety Stress Scale-21 (DASS-21): A 21-item self-report questionnaire focusing on symptoms of depression, anxiety, and stress.

- SF-12 version 2 (SF-12v2): A 12-item self-report questionnaire focusing on mental and physical functioning and impairment.

- Working Alliance Inventory- Short Form (WAI-S): A 12-item self-report questionnaire focusing on the relationship between you and the Psychology Intern providing you treatment.

- Client Satisfaction Questionnaire-8 (CSQ-8): An 8-item self-report questionnaire that focuses on satisfaction with treatment.

All procedures are part of standard clinical practices at the PTC and as such, all data will also be included in your medical record at St. Michael's Hospital. You will not be asked to do anything additional as part of this study.

\section{Potential Risks:}

Occasionally people feel uncomfortable when answering questions about their mental health, stress, or well-being or about past treatment. If you feel uncomfortable answering certain questions, you may choose not to answer those questions. Non-participation or withdrawal from this study will not in any way affect the assessment or treatment that you receive at the PTC or St. Michael's Hospital.

\section{Potential Benefits:}

It is important that patients of the PTC are being provided with effective services. Information gathered through regular evaluations will indicate whether or not patients are receiving effective assessments and treatments from the PTC provided by student learners. Information gained from this study may also add to general knowledge about providing psychological services in a Family Health Team setting.

\section{Privacy and Confidentiality:}

All persons involved in the study, including the study investigators, coordinator and delegates (hereby referred to as 'study personnel'), are committed to respecting your privacy. Information from this study will also be available to your primary care physician and other health care professionals in your circle care at St. Michael's Hospital by way of your electronic medical record. No other persons will have access to your personal health information or identifying information without your consent, unless required by law. The study personnel will make every effort to keep your personal health information private and confidential in accordance with all applicable privacy legislations, including the Personal Health Information Protection Act (PHIPA) of Ontario. Any personal health information or personal information collected about you will be 'de-identified' by replacing your personal identifying information 
with a 'study number'. The research coordinator and principal investigator here at St. Michael's Hospital are in control of the study code key, which is needed to connect your personal health information to you. The link between the study number and your personal identity will be safeguarded by the St. Michael's Hospital study personnel.

Our guidelines include the following: All information that identifies you, both paper copy and electronic information, will be kept confidential and stored and locked in a secure place that only the study personnel will be able to access. Electronic files will be stored securely on hospital or institutional networks. No study data will be sent outside of St. Michael's Hospital.

It is important to understand that despite these protections being in place, there continues to be the risk of unintentional release of information. The principal investigator will protect your records and keep all the information in your study file confidential to the greatest extent possible. The chance that this information will be accidentally released is small.

By signing this form, you are authorizing access to your medical records by the study personnel and the St. Michael's Hospital Research Ethics Board. Such access will be used only for the purpose of verifying the authenticity and accuracy of the information collected for the study, without violating your confidentiality, to the extent permitted by applicable laws and regulations.

Federal and Provincial Data Protection regulations, including the Personal Information Protection and Electronic Documents Act (PIPEDA 2000) and the Personal Health Information Protection Act (PHIPA 2004) of Ontario, protect your personal information. They also give you the right to control the use of your personal information (including personal health information) and require your written permission for this personal information to be collected, used, or disclosed for the purposes of this study, as described in this consent form. You have the right to review and copy your personal information collected in this study. However, if you decide to be in this study or choose to withdraw from it, your right to look at or copy your personal information related to this study will be delayed until after the research is completed.

The Principal Investigator will keep any personal health information about you in a secure and confidential location for 10 years and then destroy it according to St. Michael's Hospital policy. When the results of this study are published, your identity will not be disclosed.

\section{Costs and Reimbursements:}

You will not be paid to participate in this study. The study will not cost you anything to participant, and therefore no reimbursement will be available.

\section{Participation and Withdrawal:}

Participation in any research study is voluntary. If you choose not to participate, you and your family will continue to have access to customary care at St. Michael's Hospital. Your care in the PTC will not be affected. If you decide to participate in this study, you can change your mind without giving a reason, and you may withdraw from the study at any time without any effect on the care you and your family will receive at St. Michael's Hospital. Your place on the CBT wait list or your CBT sessions will not be affected if you decide that you do not want to participate, of if you withdraw from this study. You should contact a member of the study team if you choose to withdraw from participation.

Should you wish to withdraw from the study, the information about you that was collected prior to you withdrawing from the study will not be used and no new information will be collected. No new information will be collected without your permission. 


\section{New Findings:}

The study team is conducting this study to identify areas of improvement for clinical care for PTC patients. The investigators hope to inform the current practice at the PTC. We may learn new things during the study that you may need to know. We can also learn about things that might make you want to stop participating in the study. If so, you will be notified about any new information in a timely manner. You may also be asked to sign a new consent form discussing these new findings if you decide to continue in the research study.

\section{Study Contact:}

If you have any questions about assessment, student learners, or evaluations, please ask. If you have any questions, comments, or concerns later, you may contact Dr. Kelly Horner, C. Psych., (CPTC Clinical Director), at 416-864-3011 Ext. 77625.

\section{Research Ethics Board Contact:}

If you have any questions or concerns about your rights as a research participant, you may contact the Chair of the Research Ethics Board at the following institutions: St. Michael's Hospital Research Ethics Board at 416-864-6060 Ext. 2557, or the Ryerson University Research Ethics Board at 416-979-5042. 


\section{Consent Agreement}

\section{Study Title: The Effectiveness of Psychotherapy Provided by Graduate Students at an Interdisciplinary, Primary Care Clinic}

The research study has been explained to me, and my questions have been answered to my satisfaction. I have been informed of the alternatives to participation in this study. I have the right not to participate and the right to withdraw without affecting the quality of medical care at St. Michael's Hospital for me and for other members of my family. As well, the potential harms and benefits (if any) of participating in this research study have been explained to me.

I have been told that I have not waived my legal rights nor released the investigators, sponsors, or involved institutions from their legal and professional responsibilities. I know that I may ask now, or in the future, any questions I have about the study. I have been told that records relating to me and my care will be kept confidential and that no information will be disclosed without my permission unless required by law. I have been given sufficient time to read the above information.

I consent to participate. I have been told I will be given a signed copy of this consent form.

I also understand that the information collected during the research study will be included in my medical record.

Name of Participant (please print)

Signature of Participant

Date

I have explained to the above Participant the nature and purpose, the potential benefits, and possible risks associated with participation in this research study. I have answered all questions that have been raised.

Name of Individual Obtaining Informed Consent

Signature of Individual Obtaining Informed Consent

Date 


\section{Appendix B \\ Clinical Psychology Training Clinic Master Linking Log}

**Page To Be Detached By Evaluator**

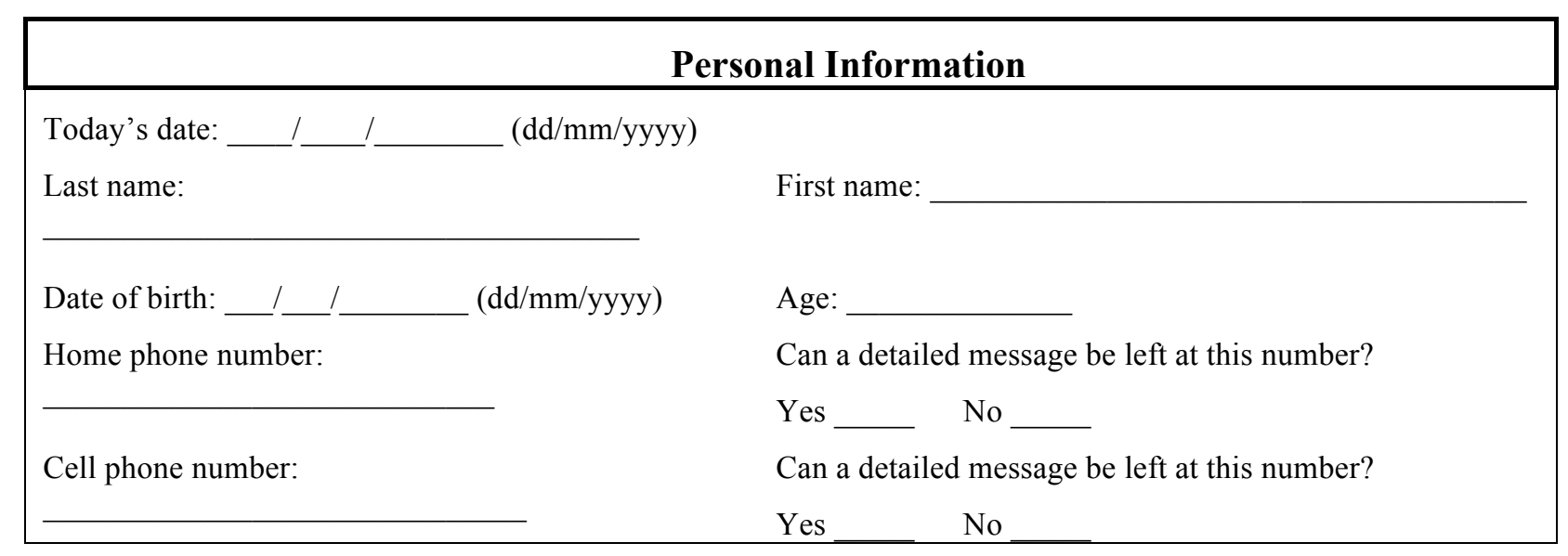

\begin{tabular}{|lc|}
\hline & In Case of Emergency Contact Information \\
\hline Name: & Relation to you: \\
\cline { 1 - 1 } & Can a detailed message be left? \\
\hline Home phone number: & Yes $\quad$ No - \\
\hline Cell phone number: & \\
\hline
\end{tabular}

\section{Quality Improvement Information}

Participant number:

Did the patient decline participation in the evaluation (as noted in the informed consent)?

Yes

$$
\text { No }
$$




\section{General Demographic Information}

What is your current relationship status? Single Long-term relationship Married Divorced/Separated Widowed Other

What sexual orientation do you most identify with?

Heterosexual/Straight Homosexual/Gay/Lesbian

Bisexual Transgender Other

Do you have any children? Yes

No

If yes, how many?:

What is your highest level of education?

Some high school High school diploma Some college/university

College/University degree Graduate education/Advance certificate or degree Other

Is there a religion or spiritual background you identify with? (e.g. Islam, Hinduism, Baha'i, Christianity, Buddhism, Atheist)

Yes If yes, please specify:

No

Is there a cultural/ethnic background you identify with? (e.g. Asian, Middle Eastern, Aboriginal, Russian, Caucasian)

Yes If yes, please specify:

No

\section{General Health Information}

Significant Past and Current Illnesses, Medical Problems, and/or Surgery

To fill this out, please consult the patient's EMR and confirm these diagnoses with the patient during the SCID. If there are discrepancies, please consult with the patient's primary care physician.

Illness/Medical Problem/Surgery

Year(s)

\section{Past/Current Mental Health Diagnoses in the EMR}

To fill this out, please consult the patient's EMR and verify these diagnoses with the patient during the SCID. If there are discrepancies, please consult with the patient's primary care physician.
Mental Illness(es)
Year(s)

\section{Past and Current Medications for Mental Health and Well-Being Purposes}

To fill this out, please consult the patient's EMR and confirm medication use with the patient during the SCID, including vitamins, herbal remedies, or over-the-counter drugs. If there are discrepancies, please consult with the patient's primary care physician.

Medication(s) and Dose

Age or Date Started /Ended

For current medications only

(Helpful? Yes/No/Somewhat)

Current Diagnoses Based on the SCID and DSM-5 
What was the reason for the patient's referral? (See referral form.)

What were the patient's diagnoses based on the DSM-5 and results of the SCID? (Please list all and include symptom severity.)

What are the patient's goals for treatment?

In what areas has the patient experienced significant stress in the past year? (Please list all applicable.)

Relationships with family or friends

Financial

Physical Health

Severe Mental Health

Housing Issues
Relationship with partner/spouse Employment/Unemployment Health of family member or close other Other, please specify:

\section{Social Support Questions}

There are people I can depend on to help me if I really need it. (Please circle one)

1 - Strongly Agree 2 - Agree 3 - Disagree 4 - Strongly Disagree

I have close relationships that provide me with a sense of emotional security and wellbeing. (Please circle one)

1 - Strongly Agree 2 - Agree 3 - Disagree 4 - Strongly Disagree

Thinking of the last two questions, whom can you turn to in your life to receive help or support?

Please list all that apply:

Please enter the scores/results of the MINI screen, OQ-45, DASS-21, SF-12, and SCID into the Session Assessment Tracking excel spreadsheet

Other Assessments Used:

Results of Other Assessments:

Recommendations:

Individual Psychotherapy with (Psychology Intern), under the supervision of

Dr. , C.Psych.

Group Treatment, please specify:

Wait List for Psychotherapy, date patient placed on wait list: (dd/mm/yyyy)

Referral to another team member (e.g. Social Work)

Referral to another community service, please specify:

Emergency Care

Other, please specify: 


\section{Appendix C \\ Outcome Questionnaire 45.2 (OQ-45)}

\section{Outcome Questionnaire $\left(\mathrm{OQ}^{\circledR}{ }^{\circledR}\right.$-45.2)}

Instructions: Looking back over the last week, including today, help us understand how you have been feeling. Read each item carefully and mark the box under the category which best describes your current situation. For this questionnaire, work is defined as employment, school, housework, volunteer work, and so forth. Please do not make any marks in the shaded areas.

$\begin{array}{lcc}\text { Name:_ Age:_ yrs. } & \text { Sex } \\ \text { ID\# } & \text { M } \square \text { F } \square\end{array}$

\begin{tabular}{|c|c|c|c|c|c|c|}
\hline Session \#_ Date _ / / & & & & & Almost & $\begin{array}{lrr}S D & I R & S R \\
\text { DO NOT } & \text { MARK BELOW }\end{array}$ \\
\hline & Never & Rarely & Sometimes & s Frequently & Always & \\
\hline 1. I get along well with others. & $\square 4$ & $\square 3$ & $\square 2$ & $\square 1$ & $\square 0$ & \\
\hline 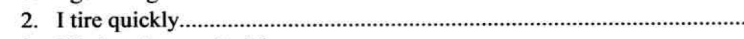 & $\ldots \square 0$ & $\square 1$ & $\square 2$ & $\square 3$ & $\square 4$ & \\
\hline 3. I feel no interest in things. & $\square 0$ & $\square 1$ & $\square 2$ & $\square 3$ & $\square 4$ & \\
\hline 4. I feel stressed at work/school... & $\ldots \square 0$ & $\square 1$ & $\square 2$ & $\square 3$ & $\square 4$ & \\
\hline 5. I blame myself for things. & $\square 0$ & $\square 1$ & $\square 2$ & $\square 3$ & $\square 4$ & \\
\hline 6. I feel irritated. &..$\square 0$ & $\square 1$ & $\square 2$ & $\square 3$ & $\square 4$ & \\
\hline 7. I feel unhappy in my marriage/significant relationship. & $\square 0$ & $\square 1$ & $\square 2$ & $\square 3$ & $\square 4$ & \\
\hline 8. I have thoughts of ending my life. & ... 0 & $\square 1$ & $\square 2$ & $\square 3$ & $\square 4$ & \\
\hline 9. I feel weak. & $\square 0$ & $\square 1$ & $\square 2$ & $\square 3$ & $\square 4$ & \\
\hline 10. I feel fearful. & $\ldots \square 0$ & $\square 1$ & $\square 2$ & $\square 3$ & $\square 4$ & \\
\hline $\begin{array}{l}\text { 11. After heavy drinking, I need a drink the next morning to get } \\
\text { going. (If you do not drink, mark "never") }\end{array}$ & $\square 0$ & $\square 1$ & $\square 2$ & $\square 3$ & $\square 4$ & \\
\hline 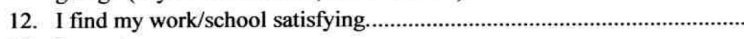 & $\ldots \square 4$ & $\square 3$ & $\square 2$ & $\square 1$ & $\square 0$ & \\
\hline 13. I am a happy person. & $\square 4$ & $\square 3$ & $\square 2$ & $\square 1$ & $\square 0$ & \\
\hline 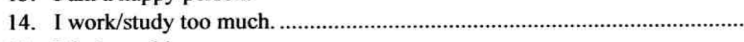 &..$\square 0$ & $\square 1$ & $\square 2$ & $\square 3$ & $\square 4$ & \\
\hline 15. I feel worthless. & $\square 0$ & $\square 1$ & $\square 2$ & $\square 3$ & $\square 4$ & \\
\hline 16. I am concerned about family troubles... &.. .00 & $\square 1$ & $\square 2$ & $\square 3$ & $\square 4$ & \\
\hline 17. I have an unfulfilling sex life. & $\square 0$ & $\square 1$ & $\square 2$ & ㅁ 3 & $\square 4$ & \\
\hline 18. I feel lonely. & ... 0 & $\square 1$ & $\square 2$ & $\square 3$ & $\square 4$ & \\
\hline 19. I have frequent arguments. & $\square 0$ & $\square 1$ & $\square 2$ & $\square 3$ & $\square 4$ & \\
\hline 20. I feel loved and wanted........ &..$\square 4$ & $\square 3$ & $\square 2$ & $\square 1$ & $\square 0$ & \\
\hline 21. I enjoy my spare time. & $\square 4$ & $\square 3$ & $\square 2$ & $\square 1$ & $\square 0$ & \\
\hline 22. I have difficulty concentrating...... &..$\square 0$ & $\square 1$ & $\square 2$ & $\square 3$ & $\square 4$ & \\
\hline 23. I feel hopeless about the future. & $\square 0$ & $\square 1$ & $\square 2$ & $\square 3$ & $\square 4$ & \\
\hline 24. I like myself. & ... 4 & $\square 3$ & $\square 2$ & $\square 1$ & $\square 0$ & \\
\hline 25. Disturbing thoughts come into my mind that I cannot get rid of. & $\square 0$ & $\square 1$ & $\square 2$ & $\square 3$ & $\square 4$ & \\
\hline $\begin{array}{l}\text { 26. I feel annoyed by people who criticize my drinking (or drug use)............. } \\
\text { (If not applicable, mark "never") }\end{array}$ &.. .00 & $\square 1$ & $\square 2$ & $\square 3$ & $\square 4$ & \\
\hline 27. I have an upset stomach. & $\square 0$ & $\square 1$ & $\square 2$ & $\square 3$ & $\square 4$ & \\
\hline 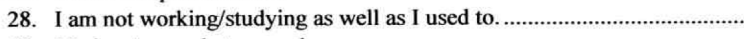 & ... 0 & $\square 1$ & $\square 2$ & $\square 3$ & $\square 4$ & \\
\hline 29. My heart pounds too much. & $\square 0$ & $\square 1$ & $\square 2$ & $\square 3$ & $\square 4$ & \\
\hline 30. I have trouble getting along with friends and close acquaintances. ......... & ... 0 & $\square 1$ & $\square 2$ & $\square 3$ & $\square 4$ & \\
\hline 31. I am satisfied with my life. & $\square 4$ & $\square 3$ & $\square 2$ & $\square 1$ & $\square 0$ & \\
\hline $\begin{array}{l}\text { 32. I have trouble at work/school because of drinking or drug use................ } \\
\text { (If not applicable, mark "never") }\end{array}$ & ... 0 & $\square 1$ & $\square 2$ & $\square 3$ & $\square 4$ & \\
\hline 33. I feel that something bad is going to happen. & $\square 0$ & $\square 1$ & $\square 2$ & $\square 3$ & $\square 4$ & \\
\hline 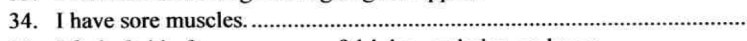 &..$\square 0$ & $\square 1$ & $\square 2$ & $\square 3$ & $\square 4$ & \\
\hline $\begin{array}{l}\text { 35. I feel afraid of open spaces, of driving, or being on buses, } \\
\text { subways, and so forth. }\end{array}$ & $\square 0$ & $\square 1$ & $\square 2$ & $\square 3$ & $\square 4$ & \\
\hline 36. I feel nervous. & 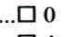 & $\square 1$ & $\square 2$ & $\square 3$ & $\square 4$ & \\
\hline 37. I feel my love relationships are full and complete. & $\square 4$ & $\square 3$ & $\square 2$ & $\square 1$ & $\square 0$ & \\
\hline 38. I feel that I am not doing well at work/school.......... & ... 00 & $\square 1$ & $\square 2$ & $\square 3$ & $\square 4$ & \\
\hline 39. I have too many disagreements at work/school. & $\square 0$ & $\square 1$ & $\square 2$ & $\square 3$ & $\square 4$ & \\
\hline 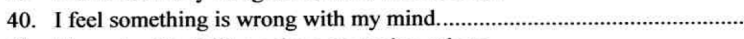 & $\square 0$ & $\square 1$ & $\square 2$ & $\square 3$ & $\square 4$ & \\
\hline 41. I have trouble falling asleep or staying asleep. & $\square 0$ & $\square 1$ & $\square 2$ & $\square 3$ & $\square 4$ & \\
\hline 42. I feel blue & ... $\square 0$ & $\square 1$ & $\square 2$ & $\square 3$ & $\square 4$ & \\
\hline 43. I am satisfied with my relationships with others. & $\square 4$ & $\square 3$ & $\square 2$ & $\square 1$ & $\square 0$ & \\
\hline 44. I feel angry enough at work/school to do something I might regret..... & ... 00 & $\square 1$ & $\square 2$ & $\square 3$ & $\square 4$ & \\
\hline 45. I have headaches. & $\square 0$ & $\square 1$ & $\square 2$ & $\square 3$ & $\square 4$ & \\
\hline \multirow{2}{*}{$\begin{array}{l}\text { Developed by Michael J. Lambert, Ph.D. and Gary M. Burlingame, Ph.D. } \\
\text { O Copyright } 1996 \text { OQ Measures LLC. } \\
\text { All Rights Reserved. License Required For All Uses. }\end{array}$} & \multirow{2}{*}{\multicolumn{5}{|c|}{$\begin{array}{l}\text { OQ MEASURES LLC } \\
\text { E-MAL: INFO@OQMEASURES.COM } \\
\text { WEB: WWW.OQMEASURES.COM } \\
\text { TOLLFRE: 1-888-MH SCORE, (1-888-647-2673) } \\
\text { FAX: 801-990-4236 }\end{array}$}} & + \\
\hline & & & & & & Total $=$ \\
\hline
\end{tabular}




\section{Appendix D \\ Depression, Anxiety, and Stress Scales-21 (DASS 21)}

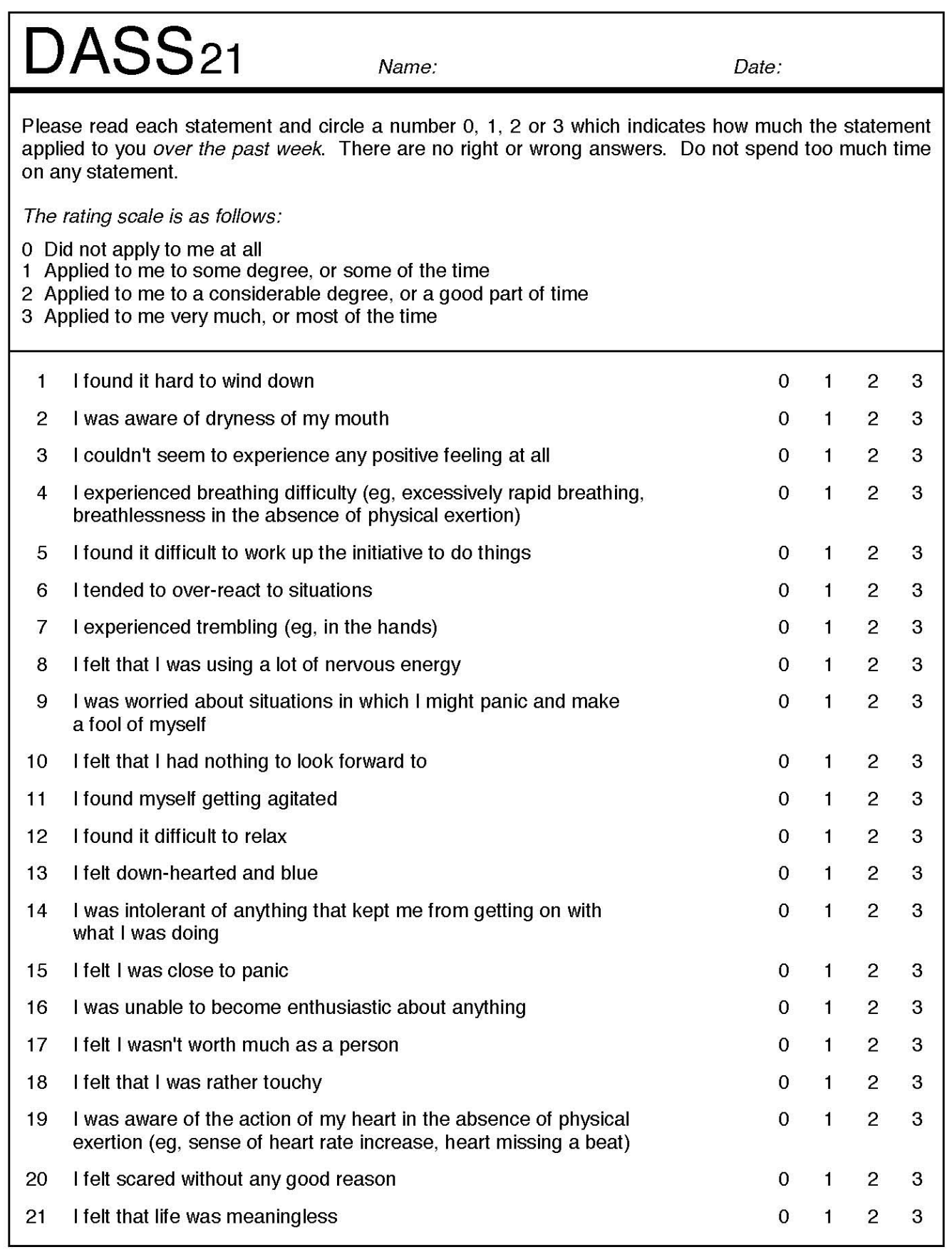




\section{Appendix E \\ Client Satisfaction Questionnaire-8 (CSQ-8)}

CSQ-8

CLIENT SATISFACTION QUESTIONNAIRE

Please help us improve our program by answering some questions about the services you have received. We are interested in your honest opinions, whether they are positive or negative. Please answer all of the questions. We also welcome your comments and suggestions. Thank you very much, we really appreciate your help.

\section{CIRCLE YOUR ANSWERS}

1. How would you rate the quaity of service you have received?
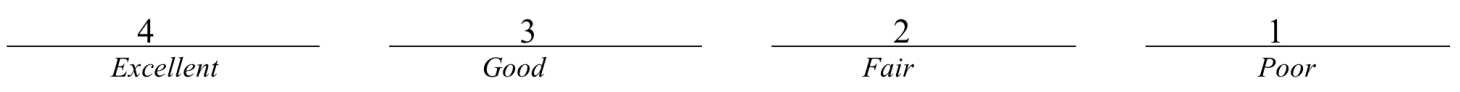

2. Did you get the kind of service you wanted?

$$
\frac{1}{\text { No, definitely not }} \quad \frac{2}{\text { No, not really }}
$$
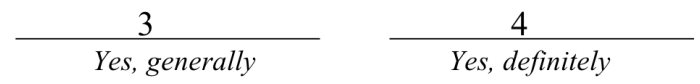

3. To what extent has our program met your needs?

$\frac{4}{\begin{array}{l}\text { Almost all of my needs } \\ \text { have been met }\end{array}}$

$\frac{3}{\text { Most of my needs }}$
have been met

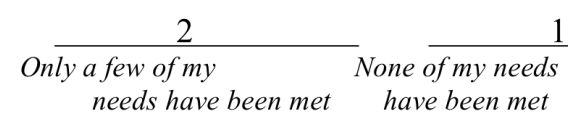

4. If a friend were in need of similar help, would you recommend our program to him or her?

$\frac{1}{\text { No, definitely not }} \quad \frac{2}{\text { No, I don't think so }} \quad \frac{3}{\text { Yes, I think so }} \quad \frac{4}{\text { Yes, definitely }}$

5. How satisfied are you with the amount of help you have received?

$\frac{1}{\text { Quite dissatisfied }} \quad \frac{2}{\begin{array}{c}\text { Indifferent or mildly } \\ \text { dissatisfied }\end{array}} \quad \frac{3}{\text { Mostly satisfied }} \quad \frac{4}{\text { Very satisfied }}$

6. Have the services you received helped you to deal more effectively with your problems?

$\begin{array}{cccc}4 & 3 & 2 & 1 \\ \begin{array}{c}\text { Yes, they helped } a \\ \text { great deal }\end{array} & \begin{array}{l}\text { Yes, they helped } \\ \text { somewhat }\end{array} & \begin{array}{l}\text { No, they really } \\ \text { didn'thelp }\end{array} & \begin{array}{l}\text { No, they seem to } \\ \text { make things worse }\end{array}\end{array}$

7. In an overall, general sense, how satisfied are you with the service you have received?

$\frac{4}{\text { Very satisfied }} \quad \frac{3}{\text { Mostly satisfied }} \quad \frac{2}{\begin{array}{c}\text { Indifferent or mildly } \\ \text { dissatisfied }\end{array}} \quad \frac{1}{\text { Quite dissatisfied }}$

8. If you were to seek help again, would you come back to our program?

$\frac{1}{\text { No, definitely not }} \quad \frac{2}{\text { No, I don't think so }} \quad \frac{3}{\text { Yes, I think so }} \quad \frac{4}{\text { Yes, definitely }}$

1989,1990, Clifford Attkisson, Ph.D. 


\section{Appendix F \\ Working Alliance Inventory-Short Form (WAI-S)}

\section{Working Alliance Inventory - Short Form - Revised}

Instructions: Below is a series of statements about experiences people might have with their therapy or therapist. Some items refer directly to your therapist with an underlined space -- as you read the sentences, mentally insert the name of your therapist in place of in the text. For each statement, please take your time to consider your own experience and then fill in the appropriate bubble.

Important: The rating scale is not the same for all the statements. PLEASE READ CAREFULLY!

1. As a result of these sessions I am clearer as to how I might be able to change.

$\begin{array}{ccccc}\text { (1) } & \text { (2) } & \text { (3) } & \text { (4) } & \text { (5) } \\ \text { Seldom } & \text { Sometimes } & \text { Fairly Often } & \text { Very Often } & \text { Always }\end{array}$

2. What I am doing in therapy gives me new ways of looking at my problem.

$\begin{array}{ccccc}\text { (1) } & \text { (2) } & \text { (3) } & \text { (4) } & \text { (5) } \\ \text { Seldom } & \text { Sometimes } & \text { Fairly Often } & \text { Very Often } & \text { Always }\end{array}$

3. I believe__ likes me.
(5)
(4)
(3)
(2)

$\begin{array}{lllll}\text { Always } & \text { Very Often } & \text { Fairly Often } & \text { Sometimes } & \text { Seldom }\end{array}$

4. ___ and I collaborate on setting goals for my therapy.

$\begin{array}{ccccc}\text { (1) } & \text { (2) } & \text { (3) } & \text { (4) } & \text { (5) } \\ \text { Seldom } & \text { Sometimes } & \text { Fairly Often } & \text { Very Often } & \text { Always }\end{array}$

5. and I respect each other.
(5)
(4)
(3)
(2)

$\begin{array}{lllll}\text { Always } & \text { Very Often } & \text { Fairly Often } & \text { Sometimes } & \text { Seldom }\end{array}$

6. ___ and I are working towards mutually agreed upon goals.
(5)
(4)
(3)
(2)
Always
Very Often
Fairly Often
Sometimes
Seldom

7. I feel that appreciates me.

$\begin{array}{ccccr}\text { (5) } & \text { (4) } & \text { (3) } & \text { (2) } & \text { (1) } \\ \text { Always } & \text { Very Often } & \text { Fairly Often } & \text { Sometimes } & \text { Seldom }\end{array}$

8. _ and I agree on what is important for me to work on.
(1)
(2)
(3)
(4)
(5)

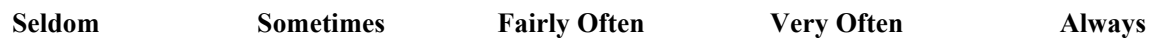

9. I feel ___ cares about me even when I do things that he/she does not approve of.
(5)
(4)
(3)
(2)
(1)

Always Very Often Fairly Often Sometimes Seldom

10. I feel that the things I do in therapy will help me to accomplish the changes that I want.

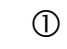

Seldom
(2)

Sometimes
(3)

Fairly Often
(4)

Very Often
(5)

Always 
11. and I have established a good understanding of the kind of changes that would be good for me.

$\begin{array}{ccccc}\text { (1) } & \text { (2) } & \text { (3) } & \text { (4) } & \text { (5) } \\ \text { Seldom } & \text { Sometimes } & \text { Fairly Often } & \text { Very Often } & \text { Always }\end{array}$

12. I believe the way we are working with my problem is correct.

(5)

Always
(4)

Very Often
(3)

Fairly Often
(2)

Sometimes
(1)

Seldom 


\section{Appendix G \\ Cognitive Therapy Scale}

\section{Cognitive Therapy Scale*}

Therapist:

Patient:

Date of session:

Tape ID Number:

Rater:

Date of rating:

Session Number:

( ) Videotape

( ) Audiotape

( ) Live Observation

Directions: For each item, assess the therapist on a scale from 0 to 6 and record the rating on the line next to the item number. Descriptions are provided for even-numbered scale points. If you believe the therapist falls between two of the descriptions, select the intervening odd number $(1,3,5)$. For example, if the therapist set a very good agenda but did not establish priorities, assign a rating of 5 rather than 4 or 6 .

If the descriptions for a given item occasionally do not seem to apply to the session you are rating, feel free to disregard them and use the more general scale below:

$\begin{array}{ccccccc}0 & 1 & 2 & 3 & 4 & 5 & 6 \\ \text { Poor } & \text { Barely } & \text { Mediocre } & \text { Satisfactory } & \text { Good } & \text { Very Good } & \text { Excellent } \\ & \text { Adequate } & & & & \end{array}$

Please do not leave any item blank. For all items, focus on the skill of the therapist, taking into account how difficult the patient seems to be.

\section{Part I. GENERAL THERAPEUTIC SKILLS}

1. AGENDA

0 Therapist did not set agenda.

2 Therapist set agenda that was vague or incomplete.

4 Therapist worked with patient to set a mutually satisfactory agenda that included specific target problems (e.g., anxiety at work, dissatisfaction with marriage).

6 Therapist worked with patient to set an appropriate agenda with target problems, suitable for the available time. Established priorities and then followed the agenda.

\section{FEEDBACK}

0 Therapist did not ask for feedback to determine patient's understanding of, or response to, the session.

2 Therapist elicited some feedback from the patient, but did not ask enough questions to be sure the patient understood the therapist's line of reasoning during the session or to ascertain whether the patient was satisfied with the session.

4 Therapist asked enough questions to be sure that the patient understood the therapist's line of reasoning throughout the session and to determine the patient's reactions to the session. The therapist adjusted his/her behavior in response to the feedback, when appropriate.

6 Therapist was especially adept at eliciting and responding to verbal and non-verbal feedback throughout the session (e.g., elicited reactions to session, regularly checked for understanding, helped summarize main points at end of session).

*This scale is designed to provide a partial evaluation of a cognitive therapist. A separate instrument is being developed to assess, in much greater detail, the quality of the therapist's conceptualization and strategy; the evaluation will be based in part on a case summary and analysis submitted by the therapist.

Furthermore, the scale is not intended to be used for the initial interview or final session with a patient. (C) 1980 J.E. Young \& A.T. Beck 


\section{UNDERSTANDING}

0 Therapist repeatedly failed to understand what the patient explicitly said and thus consistently missed the point. Poor empathic skills.

2 Therapist was usually able to reflect or rephrase what the patient explicitly said, but repeatedly failed to respond to more subtle communication. Limited ability to listen and empathize.

4 Therapist generally seemed to grasp the patient's "internal reality" as reflected by both what the patient explicitly said and what the patient communicated in more subtle ways. Good ability to listen and empathize.

6 Therapist seemed to understand the patient's "internal reality" thoroughly and was adept at communicating this understanding through appropriate verbal and non-verbal responses to the patient ( e.g. , the tone of the therapist's response conveyed a sympathetic understanding to the patient's "message"). Excellent listening and empathic skills.

\section{INTERPERSONAL EFFECTIVENESS}

0 Therapist had poor interpersonal skills. Seemed hostile, demeaning, or in some other way destructive to the patient.

2 Therapist did not seem destructive, but had significant interpersonal problems. At times, therapist appeared unnecessarily impatient, aloof, insincere, or had difficulty conveying confidence and competence.

4 Therapist displayed a satisfactory degree of warmth, concern, confidence, genuineness, and professionalism. No significant interpersonal problems.

6 Therapist displayed optimal levels of warmth, concern, confidence, genuineness, and professionalism, appropriate for this particular patient in this session.

\section{COLLABORATION}

0 Therapist did not attempt to set up a collaboration with the patient.

2 Therapist attempted to collaborate with the patient, but had difficulty either defining a problem that the patient considered important or establishing rapport.

4 Therapist was able to collaborate with patient, focus on a problem that both patient and therapist considered important, and established rapport.

6 Collaboration seemed excellent; therapist encouraged patient as much as possible to take an active role during the session (e.g., by offering choices.) so they could function as a "team".

\section{PACING AND EFFICIENT USE OF TIME}

0 Therapist made no attempt to structure therapy time. Session seemed aimless.

2 Session had some direction, but the therapist had significant problems with structuring or pacing (e.g., too little structure, in flexible about structure, too slowly paced, too rapidly paced).

4 Therapist was reasonably successful at using time efficiently. Therapist maintained appropriate control over flow of discussion and pacing.

6 Therapist used time very efficiently by tactfully limiting peripheral and unproductive discussion and by pacing the session as rapidly as was appropriate for the patient.

\section{Part II. CONCEPTUALIZATION, STRATEGY, AND TECHNIQUE}

\section{GUIDED DISCOVERY}

0 Therapist relied primarily on debate, persuasion, or "lecturing." Therapist seemed to be "cross examining" patient, putting the patient on the defensive, or forcing his/her point of view on the patient. 
2 Therapist relied too heavily on persuasion and debate, rather than a guided discovery. However, therapist's style was supportive enough that patient did not seem to feel attacked or defensive.

4 Therapist, for the most part, helped patient see new perspectives through guided discovery (e.g., examining evidence, considering alternatives, weighing advantages and disadvantages) rather than through debate. Used questioning appropriately.

6 Therapist was especially adept at using guided discovery during the session to explore problems and help patient draw his/her own conclusions. Achieved an excellent balance between skilful questioning and other modes of intervention.

\section{FOCUSING ON KEY COGNITIONS OR BEHAVIORS}

0 Therapist did not attempt to elicit specific thoughts, assumptions, images, meanings, or behaviors.

2 Therapist used appropriate techniques to elicit cognitions or behaviors; however, therapist had difficulty finding a focus or focused on cognitions/behaviors that were irrelevant to the patients key problems.

4 Therapist focused on specific cognitions or behaviors relevant to the target problem. However, therapist could have focused on more central cognitions or behaviors that offered greater promise for progress.

6 Therapist very skillfully focused on key thoughts, assumptions, behaviors, etc. That were most relevant to the problem area and offered considerable promise for progress.

9. STRATEGY FOR CHANGE (Note: For this item, focus on the quality of the therapist's strategy for change, not on how effectively the strategy was implemented or whether change actually occurred.)

$0 \quad$ Therapist did not select cognitive-behavioral techniques.

2 Therapist selected cognitive-behavioral techniques; however, either the overall strategy for bringing about change seemed vague or did not seem promising in helping the patient.

4 Therapist seemed to have a generally coherent strategy for change that showed reasonable promise and incorporated cognitive-behavioral techniques.

6 Therapist followed a consistent strategy for change that seemed very promising and incorporated the most appropriate cognitive-behavioral techniques.

10. APPLICATION OF COGNITIVE-BEHAVIORAL TECHNIQUES (Note: For this item, focus on how skillfully the techniques were applied, not on how appropriate they were for the target problem or whether change actually occurred.)

0 Therapist did not apply any cognitive-behavioral techniques.

2 Therapist used cognitive-behavioral techniques, but there were significant flaws in the way they were applied.

4 Therapist applied cognitive-behavioral techniques with moderate skill.

6 Therapist very skillfully and resourcefully employed cognitive-behavioral techniques.

\section{HOMEWORK}

0 Therapist did not attempt to incorporate homework relevant to cognitive therapy.

2 Therapist had significant difficulties incorporating homework (e.g., did not review previous homework, did not explain homework in sufficient detail, assigned inappropriate homework).

4 Therapist reviewed previous homework and assigned "standard" cognitive therapy homework generally relevant to issues dealt with in session. Homework was explained in sufficient detail.

6 Therapist reviewed previous homework and carefully assigned homework drawn from cognitive therapy for the coming week. Assignment seemed "custom tailored" to help patient incorporate new perspectives, test hypotheses, experiment with new behaviors discussed during session, etc. 
Part III. ADDITIONAL CONSIDERATIONS

12. (a) Did any special problems arise during the session (e.g., non-adherence to homework, interpersonal issues between therapist and patient, hopelessness about continuing therapy, relapse)?

YES NO

(b) If yes:

$0 \quad$ Therapist could not deal adequately with special problems that arose.

2 Therapist dealt with special problems adequately, but used strategies or conceptualizations inconsistent with cognitive therapy.

4 Therapist attempted to deal with special problems using a cognitive framework and was moderately skillful in applying techniques.

6 Therapist was very skillful at handling special problems using cognitive therapy framework.

13. Were there any significant unusual factors in this session that you feel justified the therapist's departure from the standard approach measured by this scale?

YES (Please explain below) NO

Part IV. Overall Ratings and Comments

14. How would you rate the clinician overall in this session, as a cognitive therapist:

$\begin{array}{ccccccc}0 & 1 & 2 & 3 & 4 & 5 & 6 \\ \text { Poor } & \text { Barely } & \text { Mediocre } & \text { Satisfactory } & \text { Good } & \text { Very Good } & \text { Excellent } \\ & \text { Adequate } & & & & & \end{array}$

15. If you were conducting an outcome study in cognitive therapy, do you think you would select this therapist to participate at this time (assuming this session is typical)?

$\begin{array}{ccccc}0 & 1 & 2 & 3 & 4 \\ \text { Definitely } & \text { Probably } & \text { Uncertain } & \text { Probably } & \text { Definitely } \\ \text { Not } & \text { Not } & \text { Borderline } & \text { Yes } & \text { Yes }\end{array}$

1

2

3

Moderately

4

5

6

Not difficult,

very receptive

difficult

Extremely

difficult

16. How difficult did you feel this patient was to work with?

\section{COMMENTS AND SUGGESTIONS FOR THE THERAPIST'S IMPROVEMENT:}




\section{Appendix $\mathrm{H}$}

\section{Excel Data Abstraction Form for Study 1}

\begin{tabular}{|c|c|c|c|c|c|c|c|c|c|c|}
\hline 4 & A & $\mathrm{B}$ & C & D & $E$ & $\mathrm{~F}$ & G & $\mathrm{H}$ & I & J \\
\hline 1 & & Last Name & First Name & & & & & & & \\
\hline 2 & Patient Name: & & & & & & & & & \\
\hline 3 & (Tobe detached for evaluation) & & & & & & & & & \\
\hline 4 & & & & & & & & & & \\
\hline 5 & Colour Coding: & QQ-45 & DASS-21 & Suicide Risk & $\mathrm{SF}-12 \mathrm{v} 2$ & WÁl-S & CSQ-8 & SCID & & \\
\hline 6 & & & & & & & & & & \\
\hline 7 & Participant ID (For evaluation only): & XXXX & & & & & & & & \\
\hline 8 & Patient Age: & $x \times$ & & & & & & & & \\
\hline 9 & Total \# of Individual Sessions (not including assessm & $x X$ & & & & & & & & \\
\hline 10 & Primary Manual Used: & & & & & & & & & \\
\hline 11 & Secondary Manual Used: & & & & & & & & & \\
\hline 12 & Psychology Intern for SCID & & & & & & & & & \\
\hline 13 & Attending Psychology Intern (for Treatment) & & & & & & & & & \\
\hline 14 & Final SCID & & & & & & & & & \\
\hline 15 & $\mathrm{QQ}-45$ & & & & & & & & & \\
\hline 16 & SCORING: & Symptom Distress ( & (SD) - cut off $=$ & $36,+i-10=$ reli & able change & Interpersona & ARelations| & hip $(\mathbb{R})$ - cut & $=15,+i-8=$ & liable ofi \\
\hline 17 & & Social Role - cut of & $f f=12,+1-7=$ & eliable change & & & & & & \\
\hline 18 & & Total Score - Grea & ater than $63=5$ & imilar to a patier & nt sample, less & than $63=\operatorname{Sim}$ & nilar to a cor & mmunity sam & $+i-14=r e$ & ble char \\
\hline 19 & & & & & & & & & & \\
\hline 20 & & Date (ddimmilyyyy & SD & $\mathbb{R}$ & SR & Total & 1 & 2 & 3 & 4 \\
\hline 21 & Intake Assessment & & 0 & 0 & 0 & 0 & & & & \\
\hline 22 & Meet \& Greet & & 0 & 0 & 0 & 0 & & & & \\
\hline 23 & Session 1 & & 0 & 0 & 0 & 0 & & & & \\
\hline 24 & Session 4 & & 0 & 0 & 0 & 0 & & & & \\
\hline 25 & Session 7 & & 0 & 0 & 0 & 0 & & & & \\
\hline 26 & Session 10 & & 0 & 0 & 0 & 0 & & & & \\
\hline 27 & Session 13 (may not apply) & & 0 & 0 & 0 & 0 & & & & \\
\hline 28 & Session 16 (may not apply) & & 0 & 0 & 0 & 0 & & & & \\
\hline 29 & Session 19 (may not apply) & & 0 & 0 & 0 & 0 & & & & \\
\hline 30 & Session 22 (may not apply) & & 0 & 0 & 0 & 0 & & & & \\
\hline 31 & Final Session (List Session\#) & & 0 & 0 & 0 & 0 & & & & \\
\hline 32 & QQ Total PrelPost Haitlist & & 0 & 0 & 0 & 0 & & & & \\
\hline 33 & OQ Total Change PrelPost Treatment & & 0 & 0 & 0 & 0 & & & & \\
\hline 34 & & & & & & & & & & \\
\hline 35 & DASS-21 & & & & & & & & & \\
\hline 36 & SCORING: & Depression-Norm & nal $=0-4$, Mild $=\underset{j}{0}$ & -6, Moderate= & $7-10$, Severe= & 11-13, Extreme & ly Severe= & $14+$ & & \\
\hline 37 & & Anxiety - Normal=0 & $0-3$, Mild $=4-5$ & Moderate $=6-7$ & Severe $=8-9$ & Extremely Sev & vere $=10+$ & & & \\
\hline 38 & & Stress - Normal=0- & -7, Mild $=8-9, M$ & loderate $=10-12$ & Severe $=13-1$ & 6 , Extremely S & Severe $=17+$ & & & \\
\hline 39 & & & & & & & & & & \\
\hline 40 & & Date (ddimmilyyy & Depression & Anviety & Stress & 1 & 2 & 3 & 4 & 5 \\
\hline 41 & Intake Assessment & & 0 & 0 & - & & & & & \\
\hline 42 & Meet \& Greet & & 0 & 0 & 0 & & & & & \\
\hline 43 & Session 1 & & 0 & 0 & 0 & & & & & \\
\hline 44 & Session 4 & & 0 & 0 & 0 & & & & & \\
\hline 45 & Session 7 & & 0 & 0 & 0 & 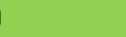 & & & & \\
\hline 46 & Session 10 & & 0 & 0 & 0 & & & & & \\
\hline 47 & Session 13 (maynot apply) & & 0 & 0 & 0 & & & & & \\
\hline 48 & Session 16 (may not apply) & & 0 & 0 & 0 & & & & & \\
\hline 49 & Session 19 (may not apply) & & 0 & 0 & 0 & & & & & \\
\hline 50 & Session 22 (may not apply) & & 0 & 0 & 0 & 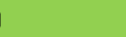 & & & & \\
\hline 51 & Final Session (List Session \#) & & 0 & 0 & 0 & & & & & \\
\hline 52 & DASS-21 Change PrelPost Waitlist & & 0 & 0 & 0 & & & & & \\
\hline 53 & DASS-21 Change PrelPost Treatment & & 0 & 0 & 0 & & & & & \\
\hline 54 & & & & & & & & & & \\
\hline 55 & Suicidality Risk Assessment (from MINN Screen & & & & & & & & & \\
\hline 56 & SCORING & Scoring: 1-8 = Low & $N, 9-16=$ Moder & ate, and $\geq 17=H$ & tigh & & & & & \\
\hline 57 & & Date (ddimmilyyyy & Total & & & & & & & \\
\hline 58 & Intake Assessment & & & & & & & & & \\
\hline 59 & Final Session (List Session\#) & & & & & & & & & \\
\hline 60 & Suicidality Risk Assessment PrelPost & & 0 & & & & & & & \\
\hline
\end{tabular}




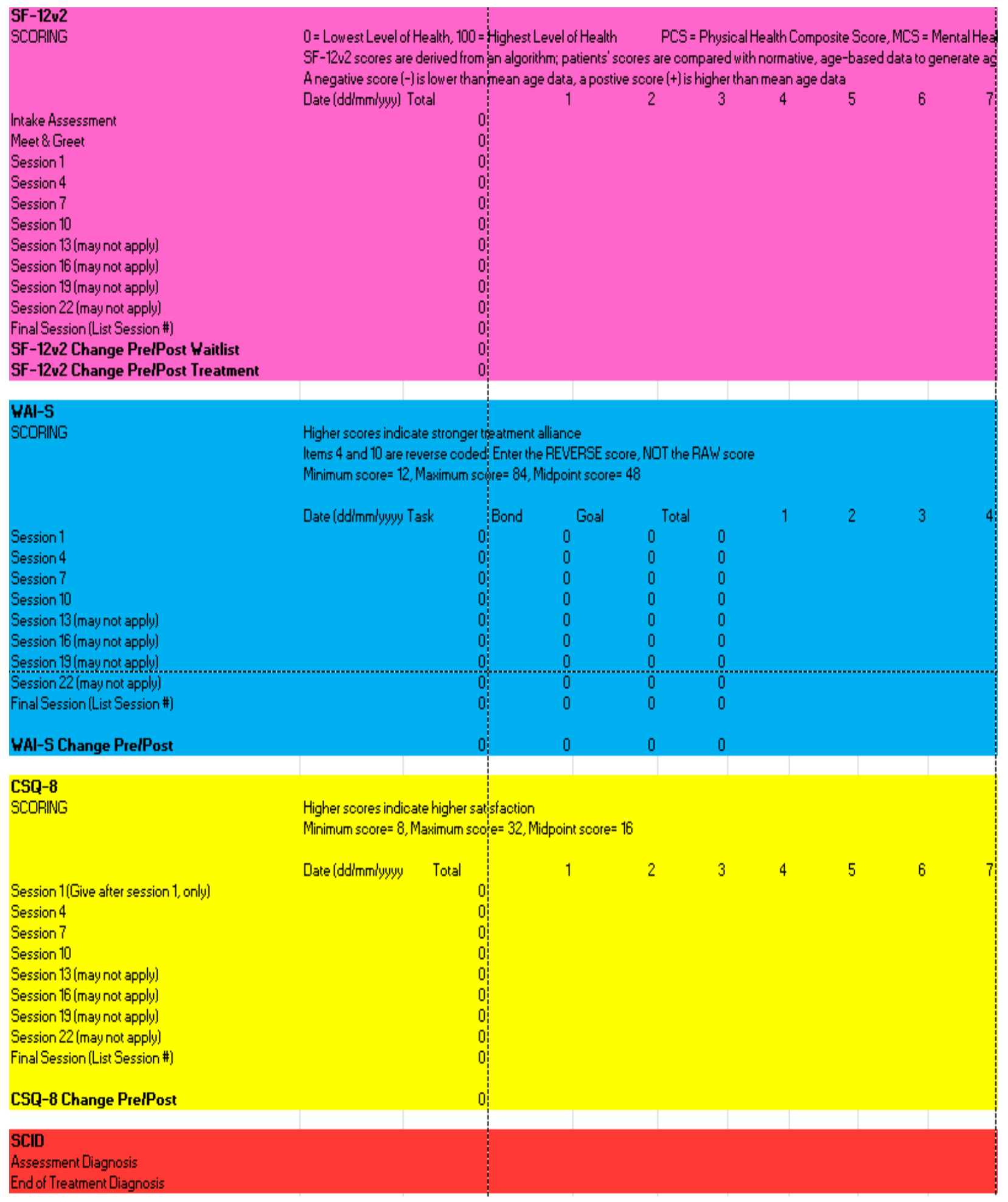


Appendix I

Psychology Training Clinic Informed Consent

\section{St. Michael's}

Inspired Care. Inspiring Science.

RYERSON

UNIVERSITY

\section{DFCM Psychology Training Clinic Information and Consent Form}

The Psychology Training Clinic's (PTC) is the result of an emergent partnership between St. Michael's Department of Family and Community Medicine and Ryerson University. The PTC services are provided by Ryerson University graduate Clinical Psychology students who are supervised by practiced clinicians registered with The College of Psychologists of Ontario.

The training clinic is committed to evidence-based practice, interprofessional team-based service delivery and training, and the use of technology to best serve patients and promote learning.

\section{Assessment and Treatment}

The PTC's primary focus is to provide the highest quality assessment and treatment services to all of our patients. Each patient is provided an initial comprehensive assessment that takes at least two meetings. Upon completion of the assessment, an in-person feedback session is provided to discuss the findings and recommendations for treatment. As part of this assessment your referring physician will also receive written feedback regarding the results of your assessment and recommendations for treatment. Less comprehensive assessments may also be done at other times during the course of treatment or at the end of treatment and serve to further inform treatment options and recommendations.

Please note that we seek to provide services for as many patients as possible. However, not all patients who are assessed are provided services at PTC. The decisions made to offer treatment are based on the types of problems reported and the availability of resources at our clinic. Those who we cannot treat directly will be offered referrals and this information will also be forwarded to your referring physician.

Treatments offered at PTC will all be short term, evidenced-based, with a primary focus in utilizing cognitive behavioural therapy (CBT). Scientific clinical outcome studies have consistently demonstrated that $\mathrm{CBT}$ is an effective form of treatment for many forms of depressive and anxiety disorders. The basic idea in CBT is to focus on the way one thinks, feels and behaves. Treatment is structured, goal-oriented, and collaborative. It is common that in-between sessions the work will continue with a focus on mutually agreed upon goals.

\section{Limits of Confidentiality}

All the information you share with the Department of Family and Community Medicine is kept within the circle of care; there are only specific times information can be shared with others outside of the circle of care.

In the following instances psychologists are required legally/ethically to report information without your consent:

1. If you as the patient were currently at risk for harming yourself.

2. If you were at risk for harming another person.

3. You disclose information that indicates a child's welfare is in danger. 
4. You disclose information that another registered health care professional in Ontario has behaved in a sexually inappropriate manner toward you.

5. If a court were to subpoena your record then your file could be forwarded to the court.

\section{Evaluation Information}

It is important to us that patients of the PTC receive quality, effective treatments, particularly in a learning environment. Like other areas of health care in Canada, to maintain a high standard of clinical practice and to meet the needs of our patient community, we routinely complete quality assessment evaluations of our services and training procedures. As such, patient data and assessment outcomes are monitored and evaluated, which is kept strictly confidential. In addition, we frequently seek feedback from our patients. For our evaluations, only collective data is analyzed and single cases are not individually reported on. Information gathered from these evaluations may be used for presentations, reports, or articles about the PTC and St. Michael's Hospital Family Health Teams. All electronic documents associated with these evaluations are encrypted and password protected.

\section{Potential Risks of Assessment and Evaluation}

Occasionally people feel uncomfortable when answering questions about their mental health, stress, or well-being or about past treatment. If you feel uncomfortable answering certain questions, you may choose not to answer those questions. As well, you may decline to have your data used for evaluation purposes at any time. Non-participation or withdrawal from evaluation will not in any way affect the assessment or treatment that you receive at the PTC or St. Michael's Hospital.

\section{Potential Benefits of Evaluation}

It is important that patients of the PTC are being provided with effective services. Information gathered through regular evaluations will indicate whether or not patients are receiving effective assessments and treatments from the PTC provided by learners. By completing the questionnaires, you may also gain a better understanding of yourself, your mental health challenges, and may make you aware of any health changes since you first began receiving services from the PTC. Information gathered from evaluations at the PTC may not only help guide policy and funding at Ryerson University and St. Michael's Hospital, but may also provide guidance to other Family Health Teams and Canada health care policy. Information gained from evaluations may also add to general knowledge about providing psychological services in a Family Health Team setting.

If you have any questions about assessment, student learners, or evaluations, please ask. If you have any questions, comments, or concerns later, you may contact Dr. Kelly Horner, C. Psych., (CPTC Clinical Director), at 416-864-3011 Ext. 77625, St. Michael's Hospital Research Ethics Board at 416-864-6060 Ext. 2557, or the Ryerson University Research Ethics Board at 416-979-5042.

\section{Consent Agreement}

Your signature below indicates that you have read the information in this agreement and have had a chance to ask any questions you have about the services provided at the PTC. Your signature also indicates that the PTC is fully affiliated with St. Michael's Hospital and that the PTC functions as an assessment, treatment, and training facility. As well, you understand that your assessment results may be used for quality assurance and evaluation purposes, though you may withdrawal your data for evaluation at any time during treatment. If you do not wish to have your data used for evaluation purposes, please check the box below. 
$\square$ I do not want my assessment data to be used for quality assurance and evaluation purposes.

You have been told that by signing this consent agreement you are not giving up any of your legal rights.

Name (please print)

Signature

Date

Witness

Date 


\title{
Appendix J \\ Informed Consent for the Online Questionnaire
}

\section{Letter of Information and Consent to Participate in a Research Study}

\author{
Study Title: Examining Perspectives on Patient Care, Interprofessional Collaboration, and Student Training \\ at the Psychology Training Clinic - Online Questionnaire
}

\author{
Study Team: \\ Principal Investigator: \\ Dr. Kelly McShane, C.Psych. \\ Ryerson University \\ 416-979-5000 Ext. 2051 \\ kmcshane@psych.ryerson.ca \\ Co-Investigators: \\ Ms. Jen Rouse, M.A. \\ Ryerson University \\ 416-979-5000 Ext. 2187 \\ jrouse@psych.ryerson.ca
}

\section{Introduction:}

Before agreeing to take part in this research study, it is important that you read the information in this research consent form. It includes details we think you need to know in order to decide if you wish to take part in the study. If you have any questions, ask a study doctor or study staff. You should not sign this form until you are sure you understand the information. All research is voluntary. Before you decide whether you will participate, it is important for you to understand why the research study is being done and what it will involve. If there is anything in this consent form that is not clear to you or that you need more information on, please ask your study doctor.

\section{Background:}

The Psychology Training Clinic's (PTC) is the result of a partnership between St. Michael's Department of Family and Community Medicine and Ryerson University. The PTC services are provided by Ryerson University graduate Clinical Psychology students (psychology interns) who are supervised by practiced clinicians registered with The College of Psychologists of Ontario.

The training clinic is committed to evidence-based practice, interprofessional team-based service delivery and training, and the use of technology to best serve patients and promote learning.

The purpose of this study is to explore patient, graduate student, and clinician perspectives on and satisfaction with the integration of the Ryerson University Psychology Training Clinic (PTC) into St. Michael's Hospital's interdisciplinary, academic family health teams (FHTs) in relation to: 1) patient care; 2) interprofessionalism (a team of professionals from different fields working together to provide patient care); and 3) the provision of psychological services by Ryerson University clinical psychology interns at the training clinic.

There will be approximately 90 participants recruited for this study from St. Michael's Hospital's FHTs and the PTC: 50 current or former PTC patients, 25 health care providers or administrators at St. Michael's FHTs, and 15 psychology interns.

\section{Description of the Research:}

You are being asked to consider participating in this research because you are either a current or former patient of the PTC, health care provider or administrator at one of St. Michael's FHTs, or psychology intern at the PTC. This research is being done in relation to a doctoral dissertation for co-investigator, Jen Rouse, M.A., at Ryerson University. 
Given Ontario's investment in FHTs and its recent push to improve mental health care in the province, it is of vital importance that patient and clinician perspectives on patient care, interprofessionalism, and student training at an academic FHT providing psychotherapy be undertaken.

The procedures that will be done for research are as follows:

- You are being asked to participate in an online questionnaire that will take approximately 20-30 minutes to complete.

- You will be directed to a specific set of questions tailored to your participant group depending on whether you identify yourself as a patient of the PTC, health care provider or administrator, or psychology intern at St. Michael's FHTs.

- $\quad$ For this survey, you will be asked to provide non-identifying demographic information and complete measures based on three factors: patient care, interprofessionalism, and interdisciplinary student education/training.

- You will be provided with a written debriefing and asked about your interest in participating in a focus group to gather more information on your perspective on patient care, interprofessionalism, and interdisciplinary student training as it relates to the PTC.

- You will be asked to provide your email and phone number should you wish to be entered into a draw for a $\$ 50$ gift card.

- You will also be offered an opportunity to participate in a focus group about your perspectives on patient care, Interprofessionalism, and student training as it relates to the PTC. Contact information for this will be provided at the end of the survey.

\section{Potential Risks:}

Occasionally people feel uncomfortable when answering questions about their health care providers, mental health treatment, peers, co-workers, or superiors, depending on your participation in the study. If you feel uncomfortable answering certain questions, you may choose not to answer those questions. Non-participation or withdrawal from this study will not in any way affect the experience that you have at the PTC or St. Michael's Hospital.

\section{Potential Benefits:}

You may not experience any direct benefits from participation. Alternatively, you may find providing valuable feedback on your experiences with the PTC to be a positive experience. This may lead to changes and improvements to better meet the needs of patients, health care providers, and student learners. Information gained from this study may also add to general knowledge about providing psychological services in a FHT setting, student training, and interprofessionalism.

\section{Privacy and Confidentiality:}

All persons involved in the study, including the study investigators, coordinator and delegates (hereby referred to as 'study personnel'), are committed to respecting your privacy. No one other than the study personnel will have access to transcripts or audio-recordings.

Our guidelines include the following: No identifying information (e.g. name, birth date) will be collected for this study to ensure your anonymity and confidentiality. All files will be kept confidential and stored and locked in a secure place that only the study personnel will be able to access. Electronic files will be stored securely on the Faculty of Arts secure server at Ryerson University. No study data will be sent outside of St. Michael's Hospital or Ryerson University. This online survey is hosted by Qualtrics, a websurvey company located in the USA and as such, is subject to U.S. laws; in particular, the US Patriot Act, which allows authorities access to the records of internet service providers. This questionnaire does not ask for personal identifiers or any information that may be used to identify you. However, if you choose to participate in the survey, you understand that your responses to the survey questions will be stored, and can be accessed, in the USA. The security and privacy policy for Qualtrics can be found at the following link: http://www.qualtrics.com/security-statement/

It is important to understand that despite these protections being in place, there continues to be the risk of unintentional release of information. The principal investigator will protect your records and keep all the information confidential to the greatest extent possible. The chance that this information will be accidentally released is small. 
The Principal Investigator will keep information linked to this study in a secure and confidential location for 10 years and then destroy it according to St. Michael's Hospital policy. When the results of this study are published, your identity will not be disclosed.

\section{Costs and Reimbursements:}

You will not be paid to participate in this study. However, if you can be entered in a draw to win a $\$ 50$ Chapters/Indigo gift cards should you decide to at the end of the study. The odds of winning a gift card are approximately 1 gift card for every 15 to 25 individuals.

\section{Participation and Withdrawal:}

Participation in any research study is voluntary. If you choose not to participate, this will in no way affect the care or working relationship you have at St. Michael's Hospital or the PTC. For patients, the care you and your family receive at St. Michael's Hospital will not be compromised based on your decision to or not to participate. For St. Michael's Hospital and Ryerson University practicum students at the PTC, your decision to participate in this study will not have any impact on your employment, professional standing, or academic evaluation. If you decide to participate in this study, you can change your mind and you may withdraw from the study at any time. You may also decline to answer any question that you may not wish to answer without penalty. However, if you do not finish the study, you will not be entered into the draw.

\section{Study Contact:}

If you have any questions about the following study, please ask. If you have any questions, comments, or concerns later, you may contact Dr. Kelly McShane, C. Psych., at 416-979-5000 Ext. 2051 or Ms. Jen Rouse, M.A., at 416979-5000 Ext. 2187.

\section{Research Ethics Board Contact:}

If you have any questions or concerns about your rights as a research participant, you may contact the Chair of the Research Ethics Board at the following institutions: St. Michael's Hospital Research Ethics Board at 416-864-6060 Ext. 2557.

\section{Consent Agreement}

Study Title: Examining Perspectives on Patient Care, Interprofessional Collaboration, and Student Training at the Psychology Training Clinic - Focus Group

The research study has been explained to me to my satisfaction. I have the right not to participate and the right to withdraw without affecting the quality of medical care or working environment at St. Michael's Hospital or the PTC. As well, the potential harms and benefits (if any) of participating in this research study have been explained to me.

I have been told that I have not waived my legal rights nor released the investigators, sponsors, or involved institutions from their legal and professional responsibilities. I know that I may ask now, or in the future, any questions I have about the study. I have been told that records relating to me will be kept confidential and that no information will be disclosed without my permission unless required by law. I have been given sufficient time to read the above information.

Yes, I choose to participate - CLICK HERE

No, I choose not to participate - PLEASE CLOSE THE WINDOW 


\title{
Appendix K \\ Written Debriefing for Study 2 Phases 1 and 2
}

\section{Study Title: Examining Perspectives on Patient Care, Interprofessional Collaboration, and Student Training at the Psychology Training Clinic}

\begin{abstract}
Background of the Study:
Ontario's mental health strategy indicates that the delivery of mental health care through interdisciplinary family health teams (FHTs) is a promising method that may improve access to psychological services. Fortunately, there have been sweeping reforms to primary care across Ontario with the establishment, many of which have included increased access to mental health services. However, this expansive growth has not been matched by research on the impact of FHTs on health and the integration of health care professionals. There is limited outcome data examining patient satisfaction, interdisciplinary team functioning, and the impact on patient care and mental health. Moreover, there is little-to-no research on the provision of psychotherapy and student training in this emerging field. Given Ontario's immense investment in FHTs and its recent push to improve mental health care in the province, it is of vital importance that patient and clinician perspectives on patient care, interprofessionalism, and student training at an academic FHT providing psychotherapy be undertaken.
\end{abstract}

The Psychology Training Clinic's (PTC) is the result of an emergent partnership between St. Michael's Hospital's Department of Family and Community Medicine and Ryerson University. The PTC services provides psychological services to patients of St. Michael's FHTs, which are provided by Ryerson University graduate Clinical Psychology students (psychology interns) who are supervised by practiced clinicians registered with The College of Psychologists of Ontario.

The training clinic is committed to evidence-based practice, interprofessional team-based service delivery and training, and the use of technology to best serve patients and promote learning. The purpose of this study is to explore patient, graduate student, and clinician perspectives on and satisfaction with the integration of the Ryerson University Psychology Training Clinic (PTC) into St. Michael's Hospital's interdisciplinary, academic FHTs in relation to: 1) patient care; 2) interprofessionalism; and 3) the provision of psychological services by Ryerson University psychology interns at the training clinic.

Contact Information: If you have any questions or concerns about this experiment or your participation in this study you may contact:

\section{Study Contact:}

If you have any questions about the following study, please ask. If you have any questions, comments, or concerns later, you may contact Dr. Kelly McShane, C. Psych., at 416-979-5000 Ext. 2051 or Ms. Jen Rouse, M.A., at 416-979-5000 Ext. 2187.

\section{Research Ethics Board Contact:}

If you have any questions or concerns about your rights as a research participant, you may contact the Chair of the Research Ethics Board at the following institutions: St. Michael's Hospital 
Research Ethics Board at 416-864-6060 Ext. 2557, or the Ryerson University Research Ethics Board at 416-979-5042.

If you would like any information about the results of the study once it is completed, please contact Jen Rouse.

\section{Thank you very much for participating!}




\section{Appendix L \\ Attitudes Toward Health Care Teams Scale (ATHCT)}

\section{Attitudes Toward Health Care Teams Scale (ATHCT)}

We would like to know about your attitudes toward interdisciplinary health care teams and the team approach to care. By interdisciplinary health care team, we mean three or more health professionals (e.g., nurse, physician, social worker) who work together and meet regularly to plan and coordinate treatment for a specific patient population. ${ }^{1}$

\begin{abstract}
"IN MY OPINION":
1.Working in teams unnecessarily complicates things most of the time
\end{abstract}

2. The team approach improves the quality of care to patients

3. Team meetings foster communication among team members from different disciplines

4. Physicians have the right to alter patient care plans developed by the team

5. Patients receiving team care are more likely than other patients to be treated as whole persons

6. A team's primary purpose is to assist physicians in achieving treatment goals for patients

7. Working on a team keeps most health professionals enthusiastic and interested in their jobs

8. Patients are less satisfied with their care when it is provided by a team

9. Developing a patient care plan with other team members avoids errors in delivering care

10. When developing interdisciplinary patient care plans, much time is wasted translating jargon from other disciplines

\begin{tabular}{llllll}
$\begin{array}{l}\text { Strongly } \\
\text { Disagree }\end{array}$ & $\begin{array}{l}\text { Moderately } \\
\text { Disagree }\end{array}$ & $\begin{array}{l}\text { Somewhat } \\
\text { Disagree }\end{array}$ & $\begin{array}{l}\text { Somewhat } \\
\text { Agree }\end{array}$ & $\begin{array}{l}\text { Moderately } \\
\text { Agree }\end{array}$ & $\begin{array}{l}\text { Strongly } \\
\text { Agree }\end{array}$ \\
\hline 0 & 0 & 0 & 0 & 0 & 0 \\
0 & 0 & 0 & 0 & 0 & 0
\end{tabular}

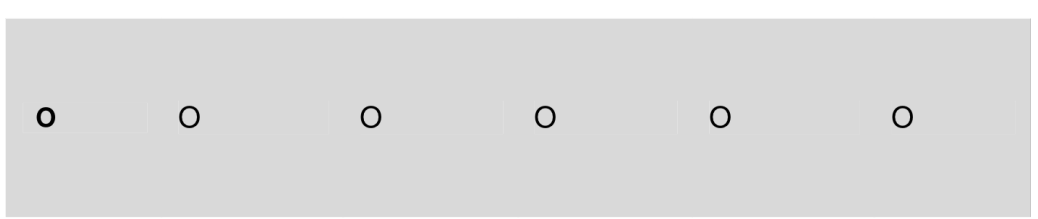
O
$\mathrm{O}$
0
$\mathrm{O}$
$\mathrm{O}$
O

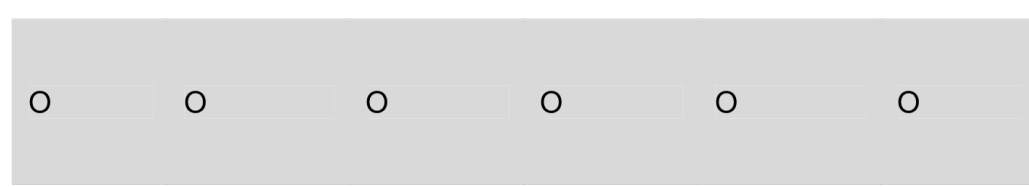

$\begin{array}{llllll}0 & 0 & 0 & 0 & 0 & 0\end{array}$

$\begin{array}{llllll}0 & 0 & 0 & 0 & 0 & 0\end{array}$

$\begin{array}{llllll}0 & 0 & 0 & 0 & 0 & 0\end{array}$

$\begin{array}{llllll}0 & 0 & 0 & 0 & 0 & 0\end{array}$

\footnotetext{
${ }^{1}$ Heinemann, Schmitt and Farrell (1994). Attitudes Towards Interdisciplinary Teams, all rights reserved
} 


\section{Appendix M \\ Interprofessional Collaboration Scale (ICS)}

Interprofessional Collaboration Scale ${ }^{3}$

(Measures communication, accommodation and isolation)

1. The team has a good understanding about their respective responsibilities.

2. Team members are usually willing to take into account the convenience of individuals when planning their work.

3. I feel that patient treatment and care are not adequately discussed between and among team members.

4. Individuals on the team share similar ideas about how to treat patients.

5. Team members are willing to discuss individuals' issues.

6. Team members cooperate with the way care is organized.

7. Team members would be willing to cooperate with new, agreed upon practices.

8. Individuals are not usually asked for their opinions.

9. Team members anticipate when they will need others' help.

10. Important information is always passed between and among team members.

11. Disagreements within the team often remain unresolved.

12. Some individuals think their work is more important than the work of others on the team.

13. Some individuals would not be willing to discuss new practices with other team members.

\begin{tabular}{|c|c|c|c|c|}
\hline $\begin{array}{l}\text { Strongly } \\
\text { Disagree }\end{array}$ & Disagree & Agree & $\begin{array}{c}\text { Strongly } \\
\text { Agree }\end{array}$ & \\
\hline 0 & 0 & 0 & 0 & IPC-C \\
\hline 0 & 0 & 0 & 0 & IPC-A \\
\hline O & 0 & 0 & 0 & IPC-C \\
\hline O & 0 & 0 & 0 & IPC-A \\
\hline O & 0 & 0 & 0 & IPC-A \\
\hline $\mathrm{O}$ & O & O & O & IPC-A \\
\hline O & 0 & 0 & 0 & IPC-A \\
\hline O & O & 0 & 0 & IPC-I \\
\hline 0 & 0 & 0 & 0 & IPC-C \\
\hline O & O & 0 & O & IPC-C \\
\hline O & O & 0 & O & IPC-C \\
\hline $\mathrm{O}$ & O & 0 & 0 & IPC-I \\
\hline 0 & 0 & 0 & 0 & IPC-I \\
\hline
\end{tabular}

IPC-C (Communication), IPC-A (Accomodation), IPC-I (Isolation)

\footnotetext{
${ }^{3}$ Kenaszchuk C, Reeves S, Nicholas D, Zwarenstein M. Validity and reliability of a multiple-group measurement scale for interprofessional collaboration. BMC Health Services Research 2010;10.
} 


\section{Appendix N \\ Interdisciplinary Education Perception Scale (IEPS)}

Using the scale below, (Strongly Disagree-1 to Strongly Agree-6) please rate your perception of your profession and other disciplines.

\begin{tabular}{|c|c|c|c|c|c|c|}
\hline DESCRIPTOR & $\begin{array}{l}\text { Strongly } \\
\text { Disagree } \\
1\end{array}$ & $\begin{array}{l}\text { Moderately } \\
\text { Disagree } \\
2\end{array}$ & $\begin{array}{l}\text { Somewhat } \\
\text { Disagree } \\
3\end{array}$ & $\begin{array}{l}\text { Somewhat } \\
\text { Agree } \\
4\end{array}$ & $\begin{array}{l}\text { Moderately } \\
\text { Agree } \\
5\end{array}$ & $\begin{array}{l}\text { Strongly } \\
\text { Agree } \\
6\end{array}$ \\
\hline $\begin{array}{l}\text { 1. Individuals in my profession are well- } \\
\text { trained. }\end{array}$ & 1 & 2 & 3 & 4 & 5 & 6 \\
\hline $\begin{array}{l}\text { 2. Individuals in my profession are able to } \\
\text { work closely with individuals in other } \\
\text { professions. }\end{array}$ & 1 & 2 & 3 & 4 & 5 & 6 \\
\hline $\begin{array}{l}\text { 3. Individuals in my profession demonstrate a } \\
\text { great deal of autonomy. }\end{array}$ & 1 & 2 & 3 & 4 & 5 & 6 \\
\hline $\begin{array}{l}\text { 4. Individuals in other professions respect the } \\
\text { work done by my profession. }\end{array}$ & 1 & 2 & 3 & 4 & 5 & 6 \\
\hline $\begin{array}{l}\text { 5. Individuals in my profession are very } \\
\text { positive about their goals and objectives. }\end{array}$ & 1 & 2 & 3 & 4 & 5 & 6 \\
\hline $\begin{array}{l}\text { 6. Individuals in my profession need to } \\
\text { cooperate with other professions. }\end{array}$ & 1 & 2 & 3 & 4 & 5 & 6 \\
\hline $\begin{array}{l}\text { 7. Individuals in my profession are very } \\
\text { positive about their contributions and } \\
\text { accomplishments. }\end{array}$ & 1 & 2 & 3 & 4 & 5 & 6 \\
\hline $\begin{array}{l}\text { 8. Individuals in my profession must depend } \\
\text { upon the work of people in other professions. }\end{array}$ & 1 & 2 & 3 & 4 & 5 & 6 \\
\hline $\begin{array}{l}\text { 9. Individuals in other professions think highly } \\
\text { of my profession. }\end{array}$ & 1 & 2 & 3 & 4 & 5 & 6 \\
\hline $\begin{array}{l}\text { 10. Individuals in my profession trust each } \\
\text { other's professional judgment. }\end{array}$ & 1 & 2 & 3 & 4 & 5 & 6 \\
\hline $\begin{array}{l}\text { 11. Individuals in my profession have a higher } \\
\text { status than individuals in other professions. }\end{array}$ & 1 & 2 & 3 & 4 & 5 & 6 \\
\hline $\begin{array}{l}\text { 12. Individuals in my profession make every } \\
\text { effort to understand the capabilities and } \\
\text { contributions of other professions. }\end{array}$ & 1 & 2 & 3 & 4 & 5 & 6 \\
\hline $\begin{array}{l}\text { 13. Individuals in my profession are extremely } \\
\text { competent. }\end{array}$ & 1 & 2 & 3 & 4 & 5 & 6 \\
\hline $\begin{array}{l}\text { 14. Individuals in my profession are willing to } \\
\text { share information and resources with other } \\
\text { professionals. }\end{array}$ & 1 & 2 & 3 & 4 & 5 & 6 \\
\hline $\begin{array}{l}\text { 15. Individuals in my profession have good } \\
\text { relations with people in other professions. }\end{array}$ & 1 & 2 & 3 & 4 & 5 & 6 \\
\hline $\begin{array}{l}\text { 16. Individuals in my profession think highly of } \\
\text { other related professions. }\end{array}$ & 1 & 2 & 3 & 4 & 5 & 6 \\
\hline $\begin{array}{l}\text { 17. Individuals in my profession work well with } \\
\text { each other. }\end{array}$ & 1 & 2 & 3 & 4 & 5 & 6 \\
\hline $\begin{array}{l}\text { 18. Individuals in other professions often seek } \\
\text { the advice of people in my profession. }\end{array}$ & 1 & 2 & 3 & 4 & 5 & 6 \\
\hline
\end{tabular}

Student IEPS - Luecht et al, (1990, Journal of Allied Health, 181-191) with permission. 


\section{Appendix O \\ Self-Efficacy for Interprofessional Experiential Learning-Revised (SEIEL-R) \\ Self-efficacy for Interprofessional Experiential Learning Revised (SEIEL-R)}

Instructions: Using the following scales, please rate your confidence in your ability to carry out some aspects of your role as a student for interprofessional learning: 1 represents very low confidence in your ability and 10 represents very high confidence in your ability.

For your reference interprofessional team refers to a team made up of individuals of different professions.

1. Working with different professionals to form a team.

$\begin{array}{lcccccccc}1 & 2 & 3 & 4 & 5 & 6 & 7 & 8 & \begin{array}{c}9 \\ \text { (Low confidence) }\end{array} \\ \text { (High confidence) }\end{array}$

2. Working with different professions to resolve problems in the team.
12
34
56
$7 \quad 8$
$\begin{array}{cr}9 & 10 \\ \text { (High confidence) }\end{array}$

3. Working with different professionals to develop a realistic appropriate patient care plan.
$\begin{array}{lc}1 & 2 \\ \text { (Low confidence) }\end{array}$
34
56
7
8
$9 \quad 10$
(Low confidence)
4
(High confidence)

4. Working with different profes
12
34
$4 \quad 56$
(Low confidence)

7

5. Working with different professionals to understand the benefits to patients of team care.
$1 \quad 2$
34
56
7
$8 \quad 9 \quad 10$
(Low conidence)

6. Understanding and discussing the objectives of interprofessional learning.
1 (Low confidence)
34
56
7
8
$\begin{array}{cr}9 & 10 \\ \text { (High confidence) }\end{array}$

7. Interacting with professionals and disciplines different from my own.
12
34
56
7
8
$\begin{array}{cr}9 & 10 \\ \text { (High confidence) }\end{array}$

8. Providing feedback to individual interprofessional team members on their function and work on the team.
$1 \quad 2$
3
$\begin{array}{lll}4 & 5 & 6\end{array}$
$7 \quad 8$
$\begin{array}{cc}9 & 10 \\ \text { (High confidence) }\end{array}$

9. Helping the patient to understand the objectives of the interprofessional learning.
$1 \quad 2$
34
56
7
8
$\begin{array}{cc}9 & 10 \\ \text { (High confidence) }\end{array}$

10.Evaluating the quality of the work as an interprofessional team.
12
3
4
56
7
8
$\begin{array}{cr}9 & 10 \\ \text { (High confidence) }\end{array}$

11.Evaluating the degree to which an interprofessional team has achieved its goals. 


$\begin{array}{lcccccccc}1 & 2 & 3 & 4 & 5 & 6 & 7 & 8 & \begin{array}{c}9 \\ \text { (Low confidence) }\end{array} \\ \text { (High confidence) }\end{array}$

12. Communicating effectively with other members of an interprofessional team.

$\begin{array}{lcccccccc}1 & 2 & 3 & 4 & 5 & 6 & 7 & 8 & \begin{array}{c}9 \\ \text { (Low confidence) }\end{array} \\ \text { (High confidence) }\end{array}$




\section{Appendix P}

Contact with Interprofessional Team Measure - Health Care Provider Version

This questionnaire reviews how much contact have you had with health care professionals, including students, other than your own within the St. Michael's Hospital Family Health Teams.

1. In the past year, how frequently did you receive consultation to another health care professional from St. Michael's Hospital Family Health Teams? (Please rate on a scale from 1 to 7 , where $1=$ Never and 7=Almost Always)

$\begin{array}{lllllll}1 & 2 & 3 & 4 & 5 & 6 & 7 \\ \text { Never } & & & & & \text { Almost Always }\end{array}$

2. In the past year, how frequently did you provide consultation to another health care professional from St. Michael's Hospital Family Health Teams? (Please rate on a scale from 1 to 7, where $1=$ Never and 7=Almost Always)

$\begin{array}{lllllll}1 & 2 & 3 & 4 & 5 & 6 & 7\end{array}$

Never Almost Always

3. In the past year, approximately what percentage of patient cases did you receive consultation from another health care professional/student within the Family Health Teams? (Does not have to equal 100\%)

$\%$ Chiropractor

$\%$ Dentist

$\%$ Dietitian

$\%$ Nurse Practitioner

$\%$ Physician

$\%$ Psychologist

$\%$ Other (Please list:

$\%$ Other (Please list:
$\%$ Counsellor (e.g. Addictions, HIV/AIDS)

$\%$ Dental Hygienist

$\%$ Nurse

$\%$ Pharmacist

$\%$ Psychiatrist

$\%$ Social Work

4. In the past year, approximately what percentage of patient cases did you provide consultation to another health care professional/student within the Family Health Teams? (Does not have to equal 100\%)

\% Chiropractor
$\%$ Dentist
$\%$ Dietitian
$\%$ Nurse Practitioner
$\%$ Physician
$\%$ Psychologist
\% Other (Please list:
\% Other (Please list:
$\%$ Counsellor (e.g. Addictions, HIV/AIDS) $\%$ Dental Hygienist $\%$ Nurse $\%$ Pharmacist $\%$ Psychiatrist $\%$ Social Work 


\section{Appendix Q \\ Contact with Interprofessional Team Measure - Patient Version}

This questionnaire reviews how much contact have you had with different health care professionals, including students, with the St. Michael's Hospital Family Health Teams.

1. How many different health care professionals have been involved in your patient care at St. Micheal's Hospital Family Health Teams (e.g. physician, nurse, nurse practitioner, chiropractor, social worker, clinical psychology student, dietitian, pharmacist, etc.)?

2. In the past year, how often did you receive care from more than one health care professional from St. Michael's Hospital Family Health Teams? (Please rate on a scale from 1 to 5, where $1=$ Never and 5=Very Often)

$\begin{array}{lllll}1 & 2 & 3 & 4 & 5 \\ \text { Never } & \text { A Few } & \text { On } & \text { Often } & \text { Very } \\ & \text { Times } & \text { Occasion } & & \text { Often }\end{array}$

3. In the past year, what health care professionals, including students, have been involved in your care at St. Michael's Hospitals Family Health Teams?

\begin{tabular}{ll} 
Chiropractor & Counsellor (e.g. Addictions, HIV/AIDS) \\
Dentist & Dental Hygienist \\
Dietitian & Nurse \\
Nurse Practitioner & Pharmacist \\
Physician & Psychiatrist \\
Psychologist & Social Work \\
Other (Please list: & \\
\hline Other (Please list: & \\
\hline
\end{tabular}




\section{Appendix R \\ Demographic Questions for Online Questionnaire}

\section{Participant Type}

Please Indicate Whether You Are a Patient, St. Michael's Hospital Family Health Team Staff, or Ryerson Psychology Practicum Student

Depending on whether you are a patient, health care provider, administrative, or reception staff, or practicum student, you will be directed to a unique set of questions.

Health Care Provider (e.g. physician, nurse, social worker) or Administrative/Reception Staff at St. Michael's Hospital's Family Health Teams

Clinical Psychology Practicum Student (past or present) at the Psychology Training Clinic

Patient (past or present) at the Psychology Training Clinic

Demographics - Health Care Provider and Administration/Reception Staff

\section{Demographics}

Gathering basic demographic information will help us better understand our data. Remember, you can skip any question that may make you feel uncomfortable answering.

Please indicate your position at St. Michael's Hospital's Family Health Teams. If you are a student/resident, please indicate the position for which you are training.

Administrative Staff

Chiropractor

Dentist

D Dietitian

C Nurse

Nurse Practitioner

Occupational Therapist

Pharmacist

C Physician

Psychiatrist

C Psychologist

Social Worker

Other (Please Indicate)
Age
18- 25
26- 30
31- 40 


$\begin{array}{ll}C & 41-50 \\ C & 51-60 \\ C & 61-70 \\ C & \text { Over } 71 \\ \text { Sex } & \\ C & \text { Male } \\ C & \text { Female } \\ C & \text { Intersex } \\ C & \text { Transgender } \\ C & \text { Other }\end{array}$

Please specify your cultural background/ethnic origin(s):

Aboriginal (e.g., First Nation, Métis, Inuit)

Arab/Middle Eastern

Asian/Island Pacific

C Black/African/Caribbean

C Hispanic/Latin American

Mixed-Ethnicity

White/European

Other

Highest level of education completed:

Some secondary (high) school, no diploma

C Secondary (high) school diploma or equivalent (e.g. GED)

Some college, CEGEP, or registered apprenticeship, no degree/certificate

College, CEGEP, or registered apprenticeship certificate/diploma

Some university credit, no degree/certificate

Bachelor's degree

Master's degree

C Professional degree (e.g. MD, LLB)

Doctoral degree

Are you co-located at the same clinic as the Psychology Training Clinic?

Yes - Psychology Training Clinic is on site (i.e. Health Centre at 80 Bond)

No - I am at a different site 


\section{Introduction:}

\section{Appendix S \\ Interview Questions}

We are here today to evaluate the Clinical Psychology Training Clinic (the PTC) in relation to mental health care delivery. In these interviews, we are hoping to gain insight into your perceptions of and experiences with the PTC in relation to patient care, collaboration among health care professionals, and the PTC as a training clinic. Only a summary of what is discussed will be presented and written up as part of the evaluation of the PTC. So, no names will be attached to specific comments and/or recommendations.

(Review and obtainment of informed consent.) To begin, we'd like to focus on patient care.

\section{Patient Care}

(1) Knowledge:

- Can you list and/or describe the mental health care resources and services provided at St. Michael's Family Health Teams?

i. Probe: The PTC might be considered one service, what services and resources does it offer to the St. Michael's Family Health Teams?

(2) Team Vision/Scope of Practice:

- When thinking about the mental health care provided at St. Michael's Hospital Family Health Teams, what role do you think the PTC is filling?

i. Probe: In what way was does the PTC compliment other services provided in St. Michael's Hospital's Family Health Team? (or duplicate?)

ii. Probe: Have you noticed any challenges with regards to the PTC and patient mental health care? (If so, what have you noticed?)

(3) Environmental Contexts and Resources:

- To what extent do physical or resource elements (e.g., location of the PTC at the Health Centre at 80 Bond, transportation difficulties) facilitate patient care with regards to services and access to services provided at the PTC?

- To what extent do physical or resource elements hinder patient care?

\section{Interprofessionalism:}

(4) Team Vision/Scope of Practice:

- When thinking about the St. Michael's Hospital Family Health Teams, including the PTC, what are your impressions on whether there is hierarchy of health care professionals versus a team-based, collaborative approach?

i. Probe: What are perceived factors that facilitate or hinder team-based functioning?

ii. Probe: Are there procedures or ways of working that foster or encourage interprofessional collaboration?

iii. What are your thoughts and experiences on the changing scopes of practice in a family health team comprised of a number of health care professionals?

(5) Communication:

- To what degree do you feel there is respect in communication and interactions between health care providers? Between providers and patients? 


\section{Training at the PTC}

(6) Knowledge:

- Tell us about your perspectives on, and experiences with the PTC as a training clinic for graduate students.

- How knowledgeable and comfortable are you with the family health team model?

(7) Competency/Confidence:

- Do you feel that graduate students have a good understanding of interdisciplinary functioning and professional roles at the St. Michael's Hospital FHTs?

i. Probe: What benefits have you experienced in relation the PTC as a training clinic?

ii. Probe: What challenges or drawbacks have you experienced in relation to the PTC as a training clinic?

iii. Probe: How confident are you in the treatment provided at the PTC by clinical psychology graduate students?

(8) Satisfaction:

- Are you satisfied with the services (psychological assessment and psychotherapy) provided by the graduate students at the PTC?

i. Probe: What aspects are you satisfied with?

ii. Probe: What aspects could be strengthened or improved? 


\title{
Appendix T \\ Informed Consent for Individual Interviews
}

\author{
Letter of Information and Consent to Participate in a Research Study
}

\section{Study Title: Examining Perspectives on Patient Care, Interprofessional Collaboration, and Student Training at the Psychology Training Clinic - Focus Group}

\section{Study Team:}

Principal Investigator:

Co-Investigators:

\author{
Dr. Kelly McShane, C.Psych. \\ Ryerson University \\ 416-979-5000 Ext. 2051 \\ kmcshane@psych.ryerson.ca
}

\author{
Ms. Jen Rouse, M.A. \\ Ryerson University \\ 416-979-5000 Ext. 2187 \\ jrouse@psych.ryerson.ca
}

\section{Introduction:}

Before agreeing to take part in this research study, it is important that you read the information in this research consent form. It includes details we think you need to know in order to decide if you wish to take part in the study. If you have any questions, ask a study doctor or study staff. You should not sign this form until you are sure you understand the information. All research is voluntary. Before you decide whether you will participate, it is important for you to understand why the research study is being done and what it will involve. If there is anything in this consent form that is not clear to you or that you need more information on, please ask your study doctor.

\section{Background:}

The Psychology Training Clinic's (PTC) is the result of a partnership between St. Michael's Department of Family and Community Medicine and Ryerson University. PTC services are provided by Ryerson University graduate Clinical Psychology students (psychology interns) who are supervised by practiced clinicians registered with The College of Psychologists of Ontario.

The training clinic is committed to evidence-based practice, interprofessional team-based service delivery and training, and the use of technology to best serve patients and promote learning.

The purpose of this study is to explore patient, psychology intern, and clinician perspectives on and satisfaction with the integration of the Ryerson University Psychology Training Clinic (PTC) into St. Michael's Hospital's interdisciplinary, academic family health teams (FHTs) in relation to: 1) patient care; 2) interprofessionalism (a team of professionals from different fields working together to provide patient care); and 3) the provision of psychological services by Ryerson University clinical psychology interns at the training clinic.

There will be approximately 65 participants recruited for this study from St. Michael's Hospital's family health teams and the PTC: 10-20 current or former PTC patients, 15-30 health care providers at St. Michael's FHTs, and $10-15$ psychology interns.

\section{Description of the Research:}

You are being asked to consider participating in this research because you are either a current or former patient of the PTC, health care provider or administrator at one of St. Michael's FHTs, or psychology intern at the PTC. This research is being done in relation to a doctoral dissertation for co-investigator, Jen Rouse, M.A., at Ryerson University. 
Given Ontario's investment in FHTs and its recent push to improve mental health care in the province, it is of vital importance that patient and clinician perspectives on patient care, interprofessionalism, and student training at an academic FHT providing psychotherapy be undertaken.

The procedures that will be done for research are as follows:

- You will be asked to participate in a one-hour audio-recorded focus group.

- Group co-facilitators will lead discussion on 3 key areas of interest: patient care, the PTC as a training clinic, and interprofessionalism.

- You will be asked to provide your perspective on six contextual factors: knowledge, environmental context and resources, team vision/scope of practice, communication, satisfaction, and competency/confidence. For example, questions will be asked about your understanding of role of the PTC within St. Michael's FHTs, elements that hinder or facilitate access to services at the PTC, your perspective on the team-based collaborative approach, and perspectives on the PTC as a training clinic for graduate students.

- Group facilitators will debrief with you at the end of the focus group.

- The audio-recordings will be transcribed and coded. Audio recording will be deleted and no identifying information will be included in the transcripts.

\section{Potential Risks:}

Occasionally people feel uncomfortable when answering questions about their health care providers, mental health treatment, peers, co-workers, or superiors, depending on your participation in the study. If you feel uncomfortable answering certain questions, you may choose not to answer those questions. Non-participation or withdrawal from this study will not in any way affect the experience that you have at the PTC or St. Michael's Hospital.

\section{Potential Benefits:}

You may not experience any direct benefits from participation. Alternatively, you may find providing valuable feedback on your experiences with the PTC to be a positive experience.. This may lead to changes and improvements to better meet the needs of patients, health care providers, and student learners. Information gained from this study may also add to general knowledge about providing psychological services in a FHT setting, student training, and interprofessionalism.

\section{Privacy and Confidentiality:}

All persons involved in the study, including the study investigators, coordinator and delegates (hereby referred to as 'study personnel'), are committed to respecting your privacy. No one other than the study personnel will have access to transcripts or audio-recordings. Please note, that your responses during the focus group cannot be kept anonymous because of the nature of the group meeting. We ask that all participants refrain from sharing the content of the discussion today with anyone else in order to ensure confidentiality of the group's discussion from others who have not participated in the group.

Our guidelines include the following: All information that identifies you, both paper copy and electronic information, will be kept confidential and stored and locked in a secure place that only the study personnel will be able to access. Electronic files will be stored securely on hospital or institutional networks. No study data will be sent outside of St. Michael's Hospital or Ryerson University.

It is important to understand that despite these protections being in place, there continues to be the risk of unintentional release of information. The principal investigator will protect your records and keep all the information confidential to the greatest extent possible. The chance that this information will be accidentally released is small.

The Principal Investigator will keep information linked to this study in a secure and confidential location for 10 years and then destroy it according to St. Michael's Hospital policy. When the results of this study are published, your identity will not be disclosed.

\section{Costs and Reimbursements:}


You will not be paid to participate in this study. The clinic will provide you with tokens to assist you with transportation costs when coming to the clinic for the focus group. As well, light refreshments will be provided at the focus group.

\section{Participation and Withdrawal:}

Participation in any research study is voluntary. If you choose not to participate, this will in no way affect the care or working relationship you have at St. Michael's Hospital or the PTC. For patients, the care you and your family receive at St. Michael's Hospital will not be compromised based on your decision to or not to participate. For St. Michael's Hospital and Ryerson University practicum students at the PTC, your decision to participate in this study will not have any impact on your employment, professional standing, or academic evaluation. If you decide to participate in this study, you can change your mind without giving a reason, and you may withdraw from the study at any time prior to the audio-recording, after which time study personnel will be unable to withdraw the information you provided. You may leave the group or decline participation at any time throughout the focus group and still receive compensation.

\section{Study Contact:}

If you have any questions about the following study, please ask. If you have any questions, comments, or concerns later, you may contact Dr. Kelly McShane, C. Psych., at 416-979-5000 Ext. 2051 or Ms. Jen Rouse, M.A., at 416979-5000 Ext. 2187.

\section{Research Ethics Board Contact:}

If you have any questions or concerns about your rights as a research participant, you may contact the Chair of the Research Ethics Board at the following institutions: St. Michael's Hospital Research Ethics Board at 416-864-6060 Ext. 2557. 


\section{Consent Agreement}

\section{Study Title: Examining Perspectives on Patient Care, Interprofessional Collaboration, and Student Training at the Psychology Training Clinic - Focus Group}

The research study has been explained to me, and my questions have been answered to my satisfaction. I have been informed of the alternatives to participation in this study. I have the right not to participate and the right to withdraw without affecting the quality of medical care or working environment at St. Michael's Hospital or the PTC. As well, the potential harms and benefits (if any) of participating in this research study have been explained to me.

I have been told that I have not waived my legal rights nor released the investigators, sponsors, or involved institutions from their legal and professional responsibilities. I know that I may ask now, or in the future, any questions I have about the study. I have been told that records relating to me and my care will be kept confidential and that no information will be disclosed without my permission unless required by law. I have been given sufficient time to read the above information.

I consent to participate. I have been told I will be given a signed copy of this consent form.

Name of Participant (please print)

Signature of Participant

Date

I have explained to the above Participant the nature and purpose, the potential benefits, and possible risks associated with participation in this research study. I have answered all questions that have been raised.

Name of Individual Obtaining Informed Consent

Signature of Individual Obtaining Informed Consent

Date 


\section{References}

Adams, A., Bond, S., \& Arber, S. (1995). Development and validation of scales to measure organisational features of acute hospital wards. International Journal of Nursing Studies, 32, 612-627. doi: 10.1016/0020-7489(95)00041-1

American Psychiatric Association. (2013). Diagnostic and statistical manual of mental disorders (5th ed.). Washington, DC: Author.

Anderson, G. L., \& Lovejoy, D. W. (2000). Predoctoral training in collaborative primary care: An exam room built for two. Professional Psychology: Research and Practice, 31, 692697. doi: 10.1037/0735-7028.31.6.692

Anderssen, E. (2015, May 22). The case for publicly funded therapy. The Globe and Mail. Retrieved from http://www.theglobeandmail.com/life/the-case-for-publicly-fundedtherapy/article24567332/

Andrews, G., Hall, W., Teeson, M., \& Henderson, S. (1999). The mental health of Australians. Canberra, Australia: Mental Health Branch, Commonwealth Department of Health and Aged Care.

Antony, M. M., Bieling, P. J., Cox, B. J., Enns, M. W., \& Swinson, R. P. (1998). Psychometric properties of the 42 -item and 21-item versions of the depression anxiety stress scales in clinical groups and a community sample. Psychological Assessment, 10, 176-181. doi: $10.1037 / 1040-3590.10 .2 .176$

Archer, J., Bower, P., Gilbody, S., Lovell, K., Richards, D., Gask, L., ... Coventry, P. (2012). Collaborative care for depression and anxiety problems (Article Number: CD006525). Cochrane Database of Systematic Reviews, 10, 1-280. doi: 10.1002/14651858.CD006525.pub2. 
Asch, S., Connor, S. E., Hamilton, E. G., \& Fox, S. A. (2000). Problems in recruiting community-based physicians for health services research. Journal of General Internal Medicine, 15(8), 591-599. doi:10.1046/j.1525-1497.2000.02329.x

Attkisson, C. C., \& Zwick, R. (1982). The client satisfaction questionnaire. Psychometric properties and correlations with service utilization and psychotherapy outcome. Evaluation and Program Planning, 5, 233-237. doi: 10.1016/0149-7189(82)90074-X

Attkisson, C. C., \& Greenfield, T. K. (2004). The UCSF client satisfaction scales: I. The Client Satisfaction Questionnaire-8. In M. Maruish (Ed.), The use of psychological testing for treatment planning and outcome assessment (3rd ed.) (pp. 799-811). Mahwah, NJ: Lawrence Erlbaum Associates.

Bandelow, B., Seidler-Brandler, U., Becker, A., Wedekind, D., \& Rüther, E. (2007). Metaanalysis of randomized controlled comparisons of psychopharmacological and psychological treatments for anxiety disorders. World Journal of Biological Psychiatry, 8(3), 175-187. doi:10.1080/15622970601110273

Barlow, D. H. (2008). Clinical handbook of psychological disorders: A step-by-step treatment manual (4th ed.). New York, NY: The Guilford Press.

Bartram, M. (2012, June 4). The mental health strategy for Canada: Leadership in health system transformation. Lecture presented at the National health Leadership Conference, Halifax, Nova Scotia. Retrieved from: www.nhlc-cnls.ca/assets/Bartram_Final.pdf

Baskerville, N. B., Hogg, W., \& Lemelin, J. (2001). Process evaluation of a tailored multifaceted approach to changing family physician practice patterns improving preventive care. The Journal of Family Practice, 50(3), 241.

Beales, J., Walji, R., Papoushek, C., Austin, Z. (2011). Exploring professional culture in the 
context of family health team interprofessional collaboration. Health and Interprofessional Practice, 1(1), 1-2. doi: 10.7772/2159-1253.1012

Bebbington, P. E., Meltzer, H., Brugha, T. S., Farrell, M., Jenkins, R., Ceresa, C., \& Lewis, G. (2000). Unequal access and unmet need: Neurotic disorders and the use of primary care services. Psychological Medicine, 30, 1359-1367. doi: 10.1017/S0033291799002950

Bernard, J. M., \& Goodyear, R. K. (2014). The fundamentals of clinical supervision (5th ed.). New York, NY: Pearson.

Bland, R. C., Newman, S. C., \& Orn, H. (1997). Help-seeking for psychiatric disorders. Canadian Journal of Psychiatry/Revue Canadienne de Psychiatrie, 42, 935-942. Retrived from https://ww1.cpa-apc.org/Publications/Archives/PDF/1997/Nov/BLAND.PDF

Blane, D., Williams, C., Morrison, J., Wilson, A., \& Mercer, S. (2013). Cognitive behavioural therapy: Why primary care should have it all. The British Journal of General Practice: The Journal of the Royal College of General Practitioners, 63(607), 103-104. doi:10.3399/bjgp13X663235

Borkovec, T. D., Newman, M. G., Pincus, A. L., \& Lytle, R. (2002). A component analysis of cognitive-behavioral therapy for generalized anxiety disorder and the role of interpersonal problems. Journal of Consulting and Clinical Psychology, 70(2), 288-298. doi:10.1037//0022-006X.70.2.288

Borrelli, B., Ogedegbe, G., Resnick, B., Orwig, D., Sepinwall, D., Ernst, D., . . Sharp, D. L. (2005). A new tool to assess treatment fidelity and evaluation of treatment fidelity across 10 years of health behavior research. Journal of Consulting and Clinical Psychology, 73, 852-860. doi: 10.1037/0022-006X.73.5.852

Borrelli, B. (2011). The assessment, monitoring, and enhancement of treatment fidelity in public 
health clinical trials. Journal of Public Health Dentistry, 71, S52-S63. doi: 10.1111/j.17527325.2011.00233.x

Boyatzis, R. E. (1998). Transforming qualitative information: Thematic analysis and code development. Thousand Oaks, CA: Sage Publications, Inc.

Bradbury, C. L., Christensen, B. K., Lau, M. A., Ruttan, L. A., Arundine, A. L., \& Green, R. E. (2008). The efficacy of cognitive behavior therapy in the treatment of emotional distress after acquired brain injury. Archives of Physical Medicine and Rehabilitation, 89(12), S61S68. doi:10.1016/j.apmr.2008.08.210

Bradley, S., \& Drapeau, M. (2014). Increasing access to mental health care through governmentfunded psychotherapy: The perspectives of clinicians. Canadian Psychology/Psychologie Canadienne, 55(2), 80-89. doi:10.1037/a0036453

Braun, A., \& Clarke, V. (2006). Using thematic analysis in psychology. Qualitative Research in Psychology, 3, 77-101. doi: 10.1191/1478088706qp063oa

Bray, J. H., Frank, R. G., McDaniel, S. H., \& Heldring, M. (2003). Education, practice and research opportunities for psychologists in primary care. In R. G. Frank, S. H. McDaniel, J. H. Bray, \& M. Heldring (Eds.), Primary care psychology (pp. 3-21). Washington, DC: APA.

Bray, J. H. (2004). Training primary care psychologists. Journal of Clinical Psychology in Medical Settings, 11, 101-107. doi: 10.1023/B:JOCS.0000025721.17763.d7

Bryan, C. J., Corso, M. L., Corso, K. A., Morrow, C. E., Kanzler, K. E., \& Ray-Sannerud, B. (2012). Severity of mental health impairment and trajectories of improvement in an integrated primary care clinic. Journal of Consulting and Clinical Psychology, 80(3), 396. doi: $10.1037 / \mathrm{a} 0027726$ 
Brown, J. B., Lewis, L., Ellis, K., Stewart, M., Freeman, T. R., \& Kasperski, M. J. (2009). Mechanisms for communicating within primary health care teams. Canadian Family Physician, 55(12), 1216-1222. Retrieved from http://www.cfp.ca/content/55/12/1216.full

Brown, J., Lewis, L., Ellis, K., Stewart, M., Freeman, T. R., \& Kasperski, M. J. (2011). Conflict on interprofessional primary health care teams - Can it be resolved? Journal of Interprofessional Care, 25(1), 4-10. doi:10.3109/13561820.2010.497750

Busija, L., Pausenberger, E., Haines, T. P., Haymes, S., Buchbinder, R., \& Osborne, R. H. (2011). Adult measures of general health and health-related quality of life. Arthritis Care \& Research, 63, S383-412. doi: 10.1002/acr.20541

Busseri, M. A., \& Tyler, J. D. (2003). Interchangeability of the working alliance inventory and working alliance inventory, short form. Psychological Assessment, 15(2), 193-197. doi:10.1037/1040-3590.15.2.193

Butler, A. C., Chapman, J. E., Forman, E. M., \& Beck, A. T. (2006). The empirical status of cognitive behavioral therapy: A review of meta-analyses. Clinical Psychology Review, 26, 17-31. doi: 10.1016/j.cpr.2005.07.003

Callard, F., Broadbent, M., Denis, M., Hotopf, M., Soncul, M., Wykes, T., ...Stewart, R. (2014). Developing a new model for patient recruitment in mental health services: A cohort study using electronic health records. British Medical Journal Open, 4(12), e005654-e005654. doi:10.1136/bmjopen-2014-005654

Canadian Psychiatric Association. (2010). Youth and mental illness. Retrieved from http://publications.cpa-apc.org/browse/documents/20

Cane, J., O'Connor, D., \& Michie, S. (2012). Validation of the theoretical domains framework for use in behaviour change and implementation research. Implementation Science, 7, 37- 
37. doi: 10.1186/1748-5908-7-37

Cape, J., Whittington, C., Buszewicz, M., Wallace, P., \& Underwood, L. (2010). Brief psychological therapies for anxiety and depression in primary care: Meta-analysis and meta-regression. BMC Medicine, 8(1), 38-38. doi:10.1186/1741-7015-8-38

Chambless, D. L., \& Ollendick, T. H. (1998). Defining empirically supported therapies. Journal of Consulting and Clinical Psychology, 66, 7-18. doi: 10.1037/0022-006X.66.1.7

Chambless, D. L., \& Ollendick, T. H. (2001). Empirically supported psychological interventions: Controversies and evidence. Annual Review of Psychology, 52, 685-716. doi: 10.1146/annurev.psych.52.1.685

Chapman, J. E. (2003). Reliability and validity of the progress questionnaire: An adaptation of the outcome questionnaire. Philadelphia, PA: Drexel University.

Chomienne, M.-H., Grenier, J., Gaboury, I., Hogg, W., Ritchie, P., \& Farmanova-Haynes, E. (2011). Family doctors and psychologists working together: Doctors' and patients' perspectives. Journal of Evaluation in Clinical Practice, 17(2), 282-287. doi: 10.1111/j.1365-2753.2010.01437.x

Chrisholm, D. (2006). Dollars, DALYs, and decisions: Economic aspects of the mental health system. Geneva, Switzerland: World Health Organization.

Chrisholm, D., Sweeny, K., Sheehan, P., Rasmussen, B., Smit, F., Cuijpers, P., \& Saxena, S. (2016). Scaling-up treatment of depression and anxiety: A global return on investment analysis. Lancet Psychiatry, 3(5), 415-424. doi: 10.1016/S2215-0366(16)30024-4

Cigrang, J. A., Dobmeyer, A. C., Becknell, M. E., Roa-Navarrete, R. A., \& Yerian, S. R. (2006). Evaluation of collaborative mental health program in primary care; Effects on patient distress and health care utilization. Primary Care and Community Psychiatry, 11(3), 121- 
127. doi: $10.1185 / 135525706 \mathrm{X} 121192$

Clatney, L., Macdonald, H., \& Shah, S. M. (2008). Mental health care in the primary care setting: Family physicians' perspectives. Canadian Family Physician Médecin De Famille Canadien, 54(6), 884-889. Retrieved from http://www.cfp.ca/content/54/6/884.full.pdf+html

Closson, T. (2008). Improving patient care for mental health and addiction services: A hospital perspective. In Psychiatric Patient Advocate Office (Ed.), Honouring the past, shaping the future: 25 years of progress in mental health advocacy and rights protection: Psychiatric Patient Advocate Office 25th anniversary report, 1983-2008 (pp. 41-43). Retrieved from http://site.ebrary.com.ezproxy.lib.ryerson.ca/lib/oculryerson/docDetail.action?docID=1027 6966

Cohen, K. R., \& Peachey, D. (2014). Access to psychological services for Canadians: Getting what works to work for Canada's mental and behavioural health. Canadian Psychology, 55(2), 126-130. doi: 10.1037/a0036499

Collier, R. (2011). Verdict still out on family health teams. Canadian Medical Association Journal, 183, 1131-1132.

Cordeiro, K., Foroughe, M., \& Mastorakos, T. (2015). Primary mental health care in the family health team setting: Tracking patient care from referral to outcome. Canadian Journal of Community Mental Health, 34(3), 1-15. doi: 10.7870/cjemh-2015-021

Craven, M. A., \& Bland, R. (2006). Better practices in collaborative mental health care: An analysis of the evidence base. Canadian Journal of Psychiatry. Revue Canadienne De Psychiatrie, 51, 1-62. Retrieved from http://www.ccmhi.ca/en/products/documents/04_BestPractices_EN.pdf 
Creswell, J. W., Fetters, M. D., \& Ivankova, N. V. (2004). Designing a mixed methods study in primary care. Annals of Family Medicine, 2, 7-12. doi: 10.1370/afm.104

Creswell, J. W. (2009). Research design: Qualitative, quantitative, and mixed methods approaches (3rd ed.). Thousand Oaks, CA: Sage Publications.

Cuijpers, P., van Straten, A., van Schaik, A., \& Andersson, G. (2009). Psychological treatment of depression in primary care: A meta-analysis. British Journal of General Practice, 59 (559), e51-e60. doi:10.3399/bjgp09X395139

Curran, V. R., Sharpe, D., \& Forristall, J. (2007). Attitudes of health sciences faculty members towards interprofessional teamwork and education. Medical Education, 41(9), 892-896. doi:10.1111/j.1365-2923.2007.02823.x

Damschroder, L. J., Aron, D. C., Keith, R. E., Kirsh, S. R., Alexander, J. A., \& Lowery, J. C. (2009). Fostering implementation of health services research findings into practice: A consolidated framework for advancing implementation science. Implementation Science: IS, 4(1), 50-50. doi:10.1186/1748-5908-4-50

Dear, B. F., Titov, N., Schwencke, G., Andrews, G., Johnston, L., Craske, M. G., \& McEvoy, P. (2011). An open trial of a brief transdiagnostic internet treatment for anxiety and depression. Behaviour Research and Therapy, 49(12), 830-837. doi:10.1016/j.brat.2011.09.007

de Winter, J. C. F, Dodou, D., \& Wieringa, P. A. (2009). Exploratory factor analysis with small sample sizes. Multivariate Behavioral Research, 44(2), 147-181. doi:10.1080/00273170902794206

Dobmeyer, A. C., Rowan, A. B., Etherage, J. R., \& Wilson, R. J. (2003). Training psychology interns in primary behavioral health care. Professional Psychology: Research and Practice, 
34, 586-594. doi: 10.1037/0735-7028.34.6.586

Dobson, K. S., Jacobson, N. S., Hollon, S. D., Dimidjian, S., Schmaling, K. B., Kohlenberg, R. J., ... Dunner, D. L. (2008). Randomized trial of behavioral activation, cognitive therapy, and antidepressant medication in the prevention of relapse and recurrence in major depression. Journal of Consulting and Clinical Psychology, 76, 468-477. doi: 10.1037/0022-006X.76.3.468

Dornan, T., Boshuizen, H., King, N., \& Scherpbier, A. (2007). Experience-based learning: A model linking the processes and outcomes of medical students' workplace learning. Medical Education, 41(1), 84-91. doi:10.1111/j.1365-2929.2006.02652.x

Douglas Williams Execute Health Program. (2012). Return patients medical health questionnaire. Gainesville, FL: Author. Retrieved from https://ufandshands.org/sites/default/files/media/douglas-williams-executivehealth/exec_health_return_patient_questionnaire.pdf

Dures, E., Rumsey, N., Morris, M., \& Gleeson, K. (2011). Mixed methods in health psychology: Theoretical and practical considerations of the third paradigm. Journal of Health Psychology, 16, 332-341. doi: 10.1177/1359105310377537

Eisenberg, D., Golberstein, E., \& Gollust, S. E. (2007). Help-seeking and access to mental health care in a university student population. Medical Care, 45, 594-601. 10.1097/MLR.0b013e31803bb4c1

Ellis, M. V. (2001). Harmful supervision, a cause for alarm: Commentary on Nelson \& Friedlander (2001) and Gray et al. (2001). Journal of Counseling Psychology, 48, 401-406. doi:10.1037/0022-0167.48.4.401

Ellis, M. V., Berger, L., Hanus, A. E., Ayala, E. E., Swords, B. A., \& Siembor, M. (2014). 
Inadequate and harmful clinical supervision: Testing a revised framework and assessing occurrence. The Counseling Psychologist, 42(4), 434-472. doi:10.1177/0011000013508656

Farmanova, E., Grenier, J., \& Chomienne, M. (2013). Pilot testing of a questionnaire for the evaluation of mental health services in family health team clinics in Ontario. Healthcare Quarterly (Toronto, Ont.), 16(4), 61. doi: 10.12927/hcq.2014.23657

Farrar, S., Kates, N., Crustolo, A. M., \& Nikolaou, L. (2001). Integrated model for mental health care. Are health care providers satisfied with it? Canadian Family Physician Médecin De Famille Canadien, 47(12), 2483-2488. Retrieved from http://www.cfp.ca/content/47/12/2483.full.pdf

Field, A. P. (2012). Discovering Statistics Using IBM SPSS Statistics: And sex and drugs and rock n' roll (4th ed.). Thousand Oaks, CA: Sage Publications Inc.

First, M. B., Williams, J. B. W., Karg, R. S., \& Spitzer, R. L. (2015). Structured clinical interview for DSM 5 Disorders-Clinician version. Arlington, VA: American Psychiatric Association.

Fletcher, J., Bassilios, B., King, K., Kohn, F., Blashki, G., Burgess, P., \& Pirkis, J. (2009). Evaluating the access to allied psychological services component of the better outcomes in mental health care program (Fourteenth interim evaluation report). Ongoing gains in improving access to mental health care in Australia. Retrieved from http://www.health.gov.au/internet/main/publishing.nsf/content/AA822EBC5AD6C339CA2 5762D001ACCB7/\$File/int14.pdf

Flottorp, S., Håvelsrud, K., \& Oxman, A. D. (2003). Process evaluation of a cluster randomized trial of tailored interventions to implement guidelines in primary care--why is it so hard to change practice? Family Practice, 20(3), 333-339. doi:10.1093/fampra/cmg316 
Frank, R., Bray, J. H., McDaniel, S. H., \& Heldring, M. (2003). Primary care psychology. Washington, DC: American Psychological Association.

Fulmer, T., Hyer, K., Flaherty, E., Mezey, M., Whitelaw, N., Jacobs, M. O., ... Pfeiffer, E. (2005). Geriatric interdisciplinary team training program: Evaluation results. Journal of Aging Health, 17, 443-470. doi: 10.1177/0898264305277962

Fouad, N. A., Grus, C. L., Hatcher, R. L., Kaslow, N. J., Hutchings, P. S., Madson, M. B., ... Crossman, R. E. (2009). Competency benchmarks: A model for understanding and measuring competence in professional psychology across training levels. Training and Education in Professional Psychology, 3(4S), S5-S26. doi:10.1037/a0015832

Fournier, L., LeSage, A. D., Toupin, J., \& Cyr, M. (1997). Telephone surveys as an alternative for estimating prevalence of mental disorders and service utilization: A Montreal catchment area study. Canadian Journal of Psychiatry, 42, 737-743. Retrieved from http://ww1.cpa-apc.org/Publications/Archives/PDF/1997/Sep/FOURNI.PDF

Gagné, M. A. (2005). Advancing the agenda for collaborative mental health care. (Report 1). Retrieved from Canadian Collaborative Mental Health Initiative website: http://www.ccmhi.ca/en/products/documents/01_AdvancingtheAgenda_EN.pdf Gagné, M., Dudgeon, S., \& Kates, N. (2006). Primary mental health care reform: Catch the wave. Healthcare Management Forum, 19, 28-33. doi: 10.1016/S0840-4704(10)60243-8

Gallagher, R. P. (2010). National survey of counseling center directors 2010. Alexandria, VA: International Association of Counseling Service.

Garcia-Shelton, L., \& Vogel, M. E. (2002). Primary care health psychology training: A collaborative model with family practice. Professional Psychology: Research and Practice, 33, 546-556. doi: 10.1037/0735-7028.33.6.546 
Garcia-Shelton, L. (2006). Meeting U.S. health care needs: A challenge to psychology.

Professional Psychology: Research and Practice, 37, 676-682. doi: 10.1037/07357028.37.6.676

Gerdes, J. L., Yuen, E. J., Wood, G. C., \& Frey, C. M. (2001). Assessing collaboration with mental health providers: The primary care perspectives. Families, Systems, \& Health, 19, 429-443. doi: 10.1037/h0089470

Goelen, G., De Clercq, G., Huyghens, L., \& Kerckhofs, E. (2006). Measuring the effect of interprofessional problem-based learning on the attitudes of undergraduate health care students. Medical Education, 40, 555-561. doi: 10.1111/j.1365-2929.2006.02478.x

Giordano, C., Arenson, C., Lyons, K. J., Collins, L., Umland, E., Smith, K., ... Rose, M. (2013). Effect of the health mentors program on student attitudes toward team care. Journal of Allied Health, 42(2), 120.

Gocan, S., Laplante, M. A., \& Woodend, A. K. (2014). Interprofessional collaboration in Ontario's family health teams: A review of the literature. Journal of Research in Interprofessional Practice and Education, 3(3), 1-19. Retrieved from http://www.cfp.ca/content/56/10/e368.full

Goldman, J., Meuser, J., Rogers, J., Lawrie, L., \& Reeves, S. (2010). Interprofessional collaboration in family health teams: An Ontario-based study. Canadian Family Physician Médecin De Famille Canadien, 56(10), e368-e374. Retrieved from http://www.cfp.ca/content/56/10/e368.full.pdf+html

Goldman, J., Meuser, J., Lawrie, L., Rogers, J., \& Reeves, S. (2010b). Interprofessional primary care protocols: A strategy to promote an evidence-based approach to teamwork and the delivery of care. Journal of Interprofessional Care, 24(6), 653-665. 
doi:10.3109/13561820903550697

Gotlib Conn, L., Reeves, S., Dainty, K., Kenaszchuk, C., \& Zwarenstein, M. (2012).

Interprofessional communication with hospitalist and consultant physicians in general internal medicine: A qualitative study. BMC Health Services Research, 12(1), 437-437. doi:10.1186/1472-6963-12-437

Gotlib Conn, L., Kenaszchuk, C., Dainty, K., Zwarenstein, M., \& Reeves, S. (2014). Nursephysician collaboration in general internal medicine: A synthesis of survey and ethnographic techniques. Health and Interprofessional Practice, 2(2), 93-99. doi:10.7772/2159-1253.1057

Grenier, J., Chomienne, M., Gaboury, I., Ritchie, P., \& Hogg, W. (2008). Collaboration between family physicians and psychologists: What do family physicians know about psychologists' work? Canadian Family Physician Médecin De Famille Canadien, 54, 232-233. Retrieved from http://pmcc.web-t.cisti.nrc.ca/picrender.cgi?artid=1281641\&blobtype=pdf

Grenier, J. (2010). Psychology on the front line of primary care. Psynopsis, 32, 3-4. Retrieved from http://www.cpa.ca/cpasite/userfiles/Documents/PsynopsisWinter2010Final.pdf

Grumbach, K., \& Bodenheimer, T. (2004). Can health care teams improve primary care practice? The Journal of the American Medical Association, 291, 1246-1251. doi: 10.1001/jama.291.10.1246

Gunn, J., William B, \& Blount, A. (2009). Primary care mental health: A new frontier for psychology. Journal of Clinical Psychology, 65, 235-252. doi: 10.1002/jclp.20499

Hall, J. G., Bainbridge, L., Buchan, A., Cribb, A., Drummond, J., Gyles, C., ...Solomon, P. (2006). A meeting of minds: Interdisciplinary research in the health sciences in Canada. Canadian Medical Association Journal/Journal De l'Association Medicale Canadienne, 
175(7), 763-771. doi:10.1503/cmaj.060783

Hameed, S. N. (2015). Psychotherapy in family medicine. Electronic Thesis and Dissertation Repository (Paper 2906). Retrieved from

http://ir.lib.uwo.ca/cgi/viewcontent.cgi?article=4416\&context=etd

Hanson, W. E., Curry, K. T., \& Bandalos, D. L. (2002). Reliability generalization of working alliance inventory scale scores. Educational and Psychological Measurement, 62(4), 659673. doi:10.1177/001316402128775076

Hatfield, D. \& Ogles, B. M. (2004). The use of outcome measures by psychologists in clinical practice. Professional Psychology: Research \& Practice, 35, 485-491. doi: 10.1037/07357028.35.5.485

Hawk, C., Buckwalter, K., Byrd, L., Cigelman, S., Dorfman, L., \& Ferguson, K. (2002). Health professions students' perceptions of interprofessional relationships. Academic Medicine, 77, 354-357. doi: 10.1097/00001888-200204000-00020

Health Canada. (2002a). A report on mental illness in Canada. (Cat. No. 0-662-32817-5). Retrieved from http://www.phac-aspc.gc.ca/publicat/miic-mmac/pdf/men_ill_e.pdf

Health Canada (2002b). The economic burden of illness in Canada, 1998. (3rd ed.). (Cat. No. H21-136/1998E). Retrieved from www.atlantique.phac.gc.ca/publicat/ebicfemc98/pdf/ebic1998.pdf

Health Care Innovation Working Group. (2012). From innovation to action: The first report of the health care working group. Retrieved from http://www.pmprovincesterritoires.ca/phocadownload/publications/health_innovation_repo rt-e-web.pdf

Heinemann, G. D., Schmitt, M. H., Farrell, M. P., \& Brallier, S. A. (1999). Development of an 
attitudes toward health care teams scale. Evaluation \& the Health Professions, 22, 123142. doi: $10.1177 / 01632789922034202$

Hofmann, S. G., Asnaani, A., Vonk, I. J. J., Sawyer, A. T., \& Fang, A. (2012). The efficacy of cognitive behavioral therapy: A review of meta-analyses. Cognitive Therapy and Research, 36(5), 427-440. doi:10.1007/s10608-012-9476-1

Horvath, A. O., \& Greenberg, L. S. (1989). Development and validation of the working alliance inventory. Journal of Counseling Psychology, 36, 223-233. doi: 10.1037/00220167.36.2.223

Horvath, A. O., \& Greenberg, L. S. (1987). Development of the Working Alliance Inventory. In L. S. Greenberg \& W. M. Pinsoff (Eds.), The psychotherapeutic process: A research handbook (pp. 529-556). New York, NY: Guilford.

Hunot, V., Churchill, R., Silva de Lima, M., \& Teixeira, V. (2007). Psychological therapies for generalised anxiety disorder (Article Number: CD006525). Cochrane Database of Systematic Reviews, 1, 1-87.

Hunsley, J. (2002). The cost-effectiveness of psychological interventions. Retrieved from http://www.cpa.ca/cpasite/userfiles/Documents/publications/Cost-Effectiveness.pdf

Hunsley, J., Elliott, K., \& Therrien, Z. (2014). The efficacy and effectiveness of psychological treatments for mood, anxiety, and related disorders. Canadian Psychology/Psychologie Canadienne, 55(3), 161-176. doi:10.1037/a0036933

Hyer, K., Fairchild, S., Abraham, I., Mezey, M., \& Fulmer, T. (2000). Measuring attitudes related to interdisciplinary training: Revisiting the Heinemann, Schmitt and Farrell 'attitudes toward health care teams' scale. Journal of Interprofessional Care, 14, 249-249. doi: $10.1080 / 713678570$ 
Jenkins, R., Bebbington, P., Brugha, T., Farrell, M., Lewis, G., \& Meltzer, H. (2003). British psychiatric morbidity survey. International Review of Psychiatry, 15, 14-18. doi: $10.1080 / 0954026021000045903$

Kainz, K. (2002). Barriers and enhancements to physician-psychologist collaboration. Professional Psychology: Research and Practice, 33, 169-175. doi: $10.1037 / 0735-7028.33 .2 .169$

Kates, N., Crustolo, A., Farrar, S., \& Nikolaou, L. (2002). Counsellors in primary care: Benefits and lessons learned. Canadian Journal of Psychiatry/Revue Canadienne De Psychiatrie, 47(9), 857-862. doi: 10.1177/070674370204700907

Kates, N. (2008). Promoting collaborative care in Canada: The Canadian collaborative mental health initiative. Families, Systems, \& Health, 26, 466-473. doi: 10.1037/a0014230

Kates, N., Gagne, M. A., \& Whyte, J. M. (2008). Collaborative mental health care in Canada: Looking back and looking ahead. Canadian Journal of Community Mental Health, 27, 1-4. Retrieved from http://www.metapress.com.ezproxy.lib.ryerson.ca/content/lk6p741156270336/fulltext.pdf

Kates, N., McPherson-Doe, C., \& George, L. (2011a). Integrating mental health services within primary care settings: The Hamilton family health team. The Journal of Ambulatory Care Management, 34(2), 174.

Kates, N., Audet, D., Mazowita, G., Lemire, F., Jayabarathan, A., Bland, R., ...Gervais, M. (2011b). The evolution of collaborative mental health care in Canada: A shared vision for the future. Canadian Journal of Psychiatry, 56(5), I1-I10.

Kazdin, A. E. (2008). Evidence-based treatment and practice: New opportunities to bridge clinical research and practice, enhance the knowledge base, and improve patient care. 
American Psychologist, 63(3), 146-159. doi:10.1037/0003-066X.63.3.146

Kenaszchuk, C., Reeves, S., Nicholas, D., \& Zwarenstein, M. (2010). Validity and reliability of a multiple-group measurement scale for interprofessional collaboration. BioMed Central Health Services Research, 10, 83-83. doi: 10.1186/1472-6963-10-83

Kenaszchuk, C., MacMillan, K., van Soeren, M., \& Reeves, S. (2011). Interprofessional simulated learning: Short-term associations between simulation and interprofessional collaboration. BioMed Central Medicine, 9, 29-29. doi: 10.1186/1741-7015-9-29

Kenaszchuk, C., Conn, L. G., Dainty, K., McCarthy, C., Reeves, S., \& Zwarenstein, M. (2012). Consensus on interprofessional collaboration in hospitals: Statistical agreement of ratings from ethnographic fieldwork and measurement scales. Journal of Evaluation in Clinical Practice, 18(1), 93-99. doi:10.1111/j.1365-2753.2010.01538.x

Kessler, R. C., Berglund, P., Demler, O., Jin, R., Merikangas, K. R., \& Walters, E. E. (2005a). Lifetime prevalence and age-of-onset distributions of DSM-IV disorders in the national comorbidity survey replication. Archives of General Psychiatry, 62, 593-602. doi: 10.1001/archpsyc.62.6.593

Kessler, R. C., Demler, O., Frank, R. G., Olfson, M., Pincus, H. A., Walters, E. E., . . . Zaslavsky, A. M. (2005b). Prevalence and treatment of mental disorders, 1990 to 2003. The New England Journal of Medicine, 352, 2515-2523. doi:

10.1056/NEJMsa043266

Kirby, M., \& Keon, W. (2006). Out of the shadows at last: Transforming mental health, mental illness and addictions services in Canada. Final report of the standing Senate Committee on Social Affairs, Science and Technology. Retrieved from http://www.parl.gc.ca/39/1/parlbus/commbus/senate/com-e/soci-e/rep-e/rep02may06-e.htm 
Kirchner, J., Edlund, C. N., Henderson, K., Daily, L., Parker, L. E., \& Fortney, J. C. (2010).

Using a multi-level approach to implement a primary care mental health (PCMH) program. Families, Systems, \& Health, 28(2), 161-174. doi:10.1037/a0020250

Kreft, I. G. G., \& De Leeuw, J. (1998). Introducing multilevel modeling. Thousand Oaks, CA: SAGE Publications, Inc. doi: 10.4135/9781849209366

Krishnamurthy, R., VandeCreek, L., Kaslow, N. J., Tazeau, Y. N., Miville, M. L., Kerns, R.. . Benton, S. A. (2004). Achieving competency in psychological assessment: Directions for education and training. Journal of Clinical Psychology, 60(7), 725-739. doi: $10.1002 /$ jclp. 20010

Ladany, N., Mori, Y., \& Mehr, K. E. (2013). Effective and ineffective supervision. The Counseling Psychologist, 41(1), 28-47. doi: 10.1177/0011000012442648

Lake, E. T. (2002). Development of the practice environment scale of the nursing work index. Research in Nursing \& Health, 25, 176-188. doi: 10.1002/nur.10032

Lambert, M. J., Burlingame, G. M., Umphress, V., Hansen, N. B., Vermeersch, D., Clouse, G. C., \& Yancher, S. C. (1996). The reliability and validity of a new psychotherapy outcome questionnaire. Clinical Psychology and Psychotherapy, 3, 249-258. doi: 10.1002/(SICI)1099-0879(199612)3:4<249::AID-CPP106>3.0.CO;2-S

Lambert, M. J., Okiishi, J. C., Finch, A. E., \& Johnson, L. D. (1998). Outcome assessment: From conceptualization to implementation. Professional Psychology: Research and Practice, 29, 63-70. doi: 10.1037/0735-7028.29.1.63

Lambert, M. J., Whipple, J. L., Vermeersch, D. A., Smart, D. W., Hawkins, E. J., Nielsen, S. L., \& Goates, M. (2002). Enhancing psychotherapy outcomes via providing feedback on client progress: A replication. Clinical Psychology \& Psychotherapy, 9(2), 91-103. 
doi:10.1002/cpp.324

Lambert, M. J., Morton, J. J., Hatfield, D., Harmon, C., Hamilton, S., Reid, R. C., Shimokawa, K., Christopherson, C., \& Burlingame, G. B. (2004). Administration and Scoring Manual for the OQ-45. Orem, UT: American Professional Credentialing Services.

Lambert, M. (2010). The outcome questionnaire system: A practical application for mental health care settings. In T. Trauer (Ed.), Outcome measurement in mental health: Theory and practice (pp.51-61). New York, NY: Cambridge University Press. doi: 10.1017/CBO9780511760686.007

Larsen, D. L., Attkisson, C. C., Hargreaves, W. A., \& Nguyen, T. D. (1979). Assessment of client/patient satisfaction: Development of a general scale. Evaluation and Program Planning, 2, 197-207. doi: 10.1016/0149-7189(79)90094-6

Leahy, R. L., Holland, S. J., \& McGinn, L. K. (2012). Treatment plans and interventions for depression and anxiety disorders (2nd ed.). New York, NY: The Guilford Press.

Lehrer, M. D., Murray, S., Benzar, R., Stormont, R., Lightfoot, M., Hafertepe, M., ... Maio, A. (2015). Peer-led problem-based learning in interprofessional education of health professions students. Medical Education Online, 20, 1-4. doi:10.3402/meo.v20.28851

Leipzig, R. M., Hyer, K., Ek, K., Wallenstein, S., Vezina, M. L., Fairchild, S., . . Howe, J. L. (2002). Attitudes toward working on interdisciplinary healthcare teams: A comparison by discipline. Journal of the American Geriatrics Society, 50, 1141-1148. doi: 10.1046/j.1532$5415.2002 .50274 . \mathrm{x}$

Lesage, A., Vasiliadis, H. M., Gagne, M.A., Dudgeon, S., Kasman, N., \& Hay, C. (2006). Prevalence of mental illness and related service utilization in Canada: An analysis of the Canadian community health survey (Canadian Collaborative Mental Health Initiative 
Report 9). Retrieved from: www.ccmhi.ca

Lim, K. L., Jacobs, P., Ohinmaa, A., Schopflocher, D., \& Dewa, C. C. (2008). A new population-based measure of the economic burden of mental illness in Canada. Chronic Diseases in Canada, 28, 92-98. Retrieved from http://www.phac-aspc.gc.ca/publicat/cdicmcbc/28-3/pdf/cdic28-3-2eng.pdf

Linde, K., Sigterman, K., Kriston, L., Rücker, G., Jamil, S., Meissner, K., \& Schneider, A. (2015). Effectiveness of psychological treatments for depressive disorders in primary care: Systematic review and meta-analysis. Annals of Family Medicine, 13(1), 56-68. doi:10.1370/afm.1719

Linden, W., Moseley, J., \& Erskine, Y. (2005). Psychology as a health-care profession: Implications for training. Canadian Psychology/Psychologie Canadienne, 46, 179-188. doi: $10.1037 / \mathrm{h} 0087025$

Lovibond, S.H. \& Lovibond, P.F. (1995a). Manual for the Depression Anxiety Stress Scales $\left(2^{\text {nd }}\right.$ ed.). Sydney, Australia: Psychology Foundation.

Lovibond, P.F. \& Lovibond, S.H. (1995b). The structure of negative emotional states: Comparison of the Depression Anxiety Stress Scales (DASS) with the Beck Depression and Anxiety Inventories. Behaviour Research and Therapy, 33, 335-343. doi: 10.1016/0005-7967(94)00075-U

Luecht, R. M., Madsen, M. K., Taugher, M. P., \& Petterson, B. J. (1990). Assessing professional perceptions: Design and validation of an Interdisciplinary Education Perception Scale. Journal of Allied Health 19(2), 181-91.

Maas, C. J. M., \& Hox, J. J. (2005). Sufficient sample sizes for multilevel modeling. Methodology, 1(3), 86-92. doi:10.1027/1614-2241.1.3.86 
Mallinckrodt, B. (1989). Social support and the effectiveness of group therapy. Journal of Counseling Psychology, 36(2), 170-175. doi:10.1037/0022-0167.36.2.170

Manca, D. P., Aubrey-Bassler, K., Kandola, K., Aguilar, C., Campbell-Scherer, D., Sopcak, N., ..., Grunfeld, E. (2014). Implementing and evaluating a program to facilitate chronic disease prevention and screening in primary care: A mixed methods program evaluation. Implementation Science: 9(1), 135-135. doi:10.1186/s13012-014-0135-7

Mann, J.J. (2005). The medical management of depression. New England Journal of Medicine, 353(17), 1819-1934. doi: 10.1056/NEJMra050730

Mann, K., McFetridge-Durdle, J., Breau, L., Clovis, J., Martin-Misener, R., Matheson, T., ... Sarria, M. (2012). Development of a scale to measure health professions students' selfefficacy beliefs in interprofessional learning. Journal of Interprofessional Care, 26, 92-99. doi: $10.3109 / 13561820.2011 .640759$

Martin, D. J., Garske, J. P., \& Davis, M. K. (2000). Relation of the therapeutic alliance with outcome and other variables: A meta-analytic review. Journal of Consulting and Clinical Psychology, 68(3), 438-450. doi:10.1037//0022-006X.68.3.438

Mason, V., Shaw, A., Wiles, N., Mulligan, J., Peters, T., Sharp, D., \& Lewis, G. (2007). GPs' experiences of primary care mental health research: A qualitative study of the barriers to recruitment. Family Practice, 24(5), 518-525. doi:10.1093/fampra/cmm047

Masters, K. S., Stillman, A. M., Browning, A. D., \& Davis, J. W. (2005). Primary care psychology training on campus: Collaboration within a student health center. Professional Psychology: Research and Practice, 36, 144-150. doi: 10.1037/0735-7028.36.2.144

Maxcy, S. (2003). Pragmatic threads in mixed methods research in the social sciences: The search for multiple modes of inquiry and the end of the philosophy of formalism. In A. 
Tashakorri \& C. Teddlie (Eds.), Handbook of mixed methods in social \& behavioral research (pp. 51-90). Los Angeles, CA: SAGE.

McDaniel, S. H., Belar, C. D., Schroeder, C., Hargrove, D. S., \& Freeman, E. L. (2002). A training curriculum for professional psychologists in primary care. Professional Psychology: Research and Practice, 33(1), 65-72. doi:10.1037//0735-7028.33.1.65

Meltzer, H., Bebbington, P., Brugha, T., Farrell, M., Jenkins, R., \& Lewis, G. (2000). The reluctance to seek treatment for neurotic disorders. Journal of Mental Health, 9, 319-327. doi: $10.1080 / \mathrm{jmh} \cdot 9.3 .319 .327$

Mental Health Commission of Canada. (2009). Toward recovery and well-being: A framework for a mental health strategy for Canada. Retrieved from http://www.mentalhealthcommission.ca/English/Pages/Strategy.aspx

Mental Health Commission of Canada. (2012a). Changing directions, changing lives: The mental health strategy for Canada. Calgary, AB: Author. Retrieved from: strategy.mentalhealthcommission.ca/pdf/strategy-images-en.pdf

Mental Health Commission of Canada. (2012b). Why investing in mental health will contribute to Canada's economic prosperity and to the sustainability of our health care system. Retrieved from: strategy.mentalhealthcommission.ca/pdf/case-for-investment-en.pdf

Michie, S., Johnston, M., Abraham, C., Lawton, R., Parker, D., Walker, A., \& Psychological Theory Group. (2005). Making psychological theory useful for implementing evidence based practice: A consensus approach. Quality \& Safety in Health Care, 14, 26-33. doi: 10.1136/qshc.2004.011155

Miller, S. D., Duncan, B. L., Brown, J., Sparks, J. A., \& Claud, D. A. (2003). The outcome rating scale: A preliminary study of the reliability, validity, and feasibility of a brief visual analog 
measure. Journal of Brief Therapy, 2, 91-100. Retrieved from

http://www.scottdmiller.com/uploadedFiles/JBTORSReplication.pdf

Mohr, D. C., Hart, S. L., Howard, I., Julian, L., Vella, L., Catledge, C., \& Feldman, M. D. (2006). Barriers to psychotherapy among depressed and nondepressed primary care patients. Annals of Behavioral Medicine, 32, 254-258. doi:

10.1207/s15324796abm3203_12

Moore R, Mao Y, Zhang J, Clarke K, \& Laboratory Centre for Disease Control. (1997). Economic burden of illness in Canada, 1993. Ottawa, ON: Canadian Public Health Association.

Moritsugu, J., Wong, F. Y., \& Duffy, K. G. (2010). Community psychology (4th ed.) Boston, MA: Allyn \& Bacon.

Moulding, R., Grenier, J., Blashki, G., Ritchie, P., Pirkis, J., \& Chomienne, M. H. (2009). Integrating psychologists into the Canadian health care system: The example of Australia. Canadian Journal of Public Health/Revue Canadienne De Santé Publique, 100(2), 145147.

Mowbray, C. T., Megivern, D., Mandiberg, J. M., Strauss, S., Stein, C. H., Collins, K.,... Lett, R. (2006). Campus mental health services: Recommendations for change. American Journal of Orthopsychiatry, 76, 226-237. doi: 10.1037/0002-9432.76.2.226

Mu, K., Chao, C. C., Jensen, G. M., \& Royeen, C. B. (2004). Effects of interprofessional rural training on students' perceptions of interprofessional health care services. Journal of Allied Health, 33(2), 125-131. 
Mueller, R. M., Lambert, M. J, \& Burlingame, G. M. (1998). Construct validity of the outcome questionnaire: A confirmatory factor analysis. Journal of Personality Assessment, 70, 248-262. doi: 10.1207/s15327752jpa7002_5

Mulvale, G. (2006). Mental health policy and service delivery in Canada: Issues in policy reform, access to care, and use of mental health providers. (Doctoral dissertation, McMaster University). (2007-99240-064). Retrieved from Dissertation Abstracts International. Section B: The Sciences and Engineering.

Mulvale, G., Abelson, J., \& Goering, P. (2007). Mental health service delivery in Ontario, Canada: How do policy legacies shape prospects for reform? Health Economics, Policy, and Law, 2, 363-389. doi: 10.1017/S1744133107004318

Mulvale, G., \& Bourgeault, I. L. (2007). Finding the right mix: How do contextual factors affect collaborative mental health care in Ontario? Canadian Public Policy/Analyse De Politiques, 33, S49-S64. doi: 10.3138/H400-0370-L1P4-K804

Mulvale, G., Danner, U., \& Pasic, D. (2008). Advancing community-based collaborative mental health care through interdisciplinary family health teams in Ontario. Canadian Journal of Community Health, 27, 55-73.

Myhr, G., \& Payne, K. (2006). Cost-effectiveness of cognitive-behavioural therapy for mental disorders: Implication for public health care funding policy in Canada. Canadian Journal of Psychiatry, 51, 662-670. doi: 10.1177/070674370605101006

Nash, J. M., McKay, K. M., Vogel, M. E., \& Masters, K. S. (2012). Functional roles and foundational characteristics of psychologists in integrated primary care. Journal of Clinical Psychology in Medical Settings, 19(1), 93-104. doi:10.1007/s10880-011-9290-z

Neill, M., Hayward, K. S., \& Peterson, T. (2007). Students' perceptions of the interprofessional 
team in practice through the application of servant leadership principles. Journal of Interprofessional Care, 21, 425-425. doi: 10.1080/13561820701443512

Nelson, M. L., \& Friedlander, M. L. (2001). A close look at conflictual supervisory relationships: The trainee's perspective. Journal of Counseling Psychology, 48(4), 384-395. doi:10.1037/0022-0167.48.4.384

Nieuwsma, J. A., Trivedi, R. B., McDuffie, J., Kronish, I., Benjamin, D., \& Williams, J. W. (2012). Brief psychotherapy for depression: A systematic review and meta-analysis. The International Journal of Psychiatry in Medicine, 43(2), 129-151. doi:10.2190/PM.43.2.c

Nyman, S. J., Nafziger, M. A., \& Smith, T. B. (2010). Client outcomes across counselor training level within a multitiered supervision model. Journal of Counseling \& Development, 88(2), 204-209. doi:10.1002/j.1556-6678.2010.tb00010.x

Ontario Ministry of Health and Long-Term Care. (2005a). Guide to interdisciplinary team roles and responsibilities. Family health teams. Advancing primary health care. Retrieved from http://www.health.gov.on.ca/en/pro/programs/fht/docs/fht_inter_team.pdf

Ontario Ministry of Health and Long-Term Care. (2005b). Guide to collaborative team practice. Family health teams. Advancing primary health care. Retrieved from http://www.health.gov.on.ca/en/pro/programs/fht/docs/fht_collab_team.pdf

Ontario Ministry of Health and Long-Term Care. (2011). Open minds, health minds. Ontario's comprehensive mental health and addictions strategy. Retrieved from http://www.health.gov.on.ca/en/public/publications/ministry_reports/mental_health2011/m entalhealth_rep2011.pdf

Ontario Ministry of Health and Long Term Care. (2016, January 22). Family Health Teams. Retrieved from http://www.health.gov.on.ca/en/pro/programs/fht/ 
Ontario Psychological Association Working Group on Clinical Supervision. (2015). OPA Supervisee Bill of Rights. Toronto, ON: Ontario Psychological Association. Retrieved from http://www.psych.on.ca/OPA/media/opa-main/Board-Members/OPA-Bill-of-Rights-forSupervisees-Final.pdf?ext=.pdf

Olatunji, B. O., Davis, M. L., Powers, M. B., \& Smits, J. A. J. (2013). Cognitive-behavioral therapy for obsessive-compulsive disorder: A meta-analysis of treatment outcome and moderators. Journal of Psychiatric Research, 47, 33. doi: 10.1016/j.jpsychires.2012.08.020

Oza, S. K., Boscardin, C. K., Wamsley, M., Sznewajs, A., May, W., Nevins, A., ... Hauer, K. E. (2015). Assessing 3rd year medical students' interprofessional collaborative practice behaviors during a standardized patient encounter: A multi-institutional, cross-sectional study. Medical Teacher, 37(10), 915. doi: 10.3109/0142159X.2014.970628

Parkinson, A., Jorm, L., Douglas, K. A., Gee, A., Sargent, G. M., Lujic, S., \& McRae, I. S. (2015). Recruiting general practitioners for surveys: Reflections on the difficulties and some lessons learned. Australian Journal of Primary Health, 21(2), 254. doi:10.1071/PY13129

Patton, M. Q. (2002). Qualitative research and evaluation methods (3rd ed.). Thousand Oaks, CA: Sage Publications, Inc.

Peachey, D., Hicks, V., \& Adams, O. (2013). An imperative for change: Access to psychological services for Canada. Retrieved from http://www.cpa.ca/docs/File/Position/An_Imperative_for_Change.pdf Pingitore, D. P. (1999). Postdoctoral training in primary care health psychology: Duties, observations, and recommendations. Professional Psychology: Research and Practice, 30, 283-290. doi: 10.1037/0735-7028.30.3.283 
Pinto, A., Lee, S., Lombardo, S., Salama, M., Ellis, S., Kay, T., ... Landry, M. D. (2012). The impact of structured inter-professional education on health care professional students' perceptions of collaboration in a clinical setting. Physiotherapy Canada/Physiothérapie Canada, 64(2), 145-156. doi:10.3138/ptc.2010-52

Pustejovsky, J. E., Hedges, L. V., \& Shadish, W. R. (2014). Design-comparable effect sizes in multiple baseline designs: A general modeling framework. Journal of Educational and Behavioral Statistics, 39(5), 368-393. doi: 10.3102/1076998614547577

Ragaz, N., Berk, A., Ford, D., \& Morgan, M. (2010). Strategies for family health team leadership: Lessons learned by successful teams. Healthcare Quarterly, 13(3), 39-43. doi:10.12927/hcq.2010.21814

Rakovshik, S. G., \& McManus, F. (2010). Establishing evidence-based training in cognitive behavioral therapy: A review of current empirical findings and theoretical guidance. Clinical Psychology Review, 30(5), 496-516. doi:10.1016/j.cpr.2010.03.004

Raudenbush, S. W., \& Bryk, A. S. (2002). Hierarchical linear models: Applications and data analysis methods (2nd ed.). Thousand Oaks, CA: Sage Publications, Inc.

Richter-Sundberg, L., Nyström, M. E., Krakau, I., Sandahl, C. (2015). Improving treatment of depression in primary health care: A case study of obstacles to perform a clinical trial designed to implement practice guidelines. Primary Health Care Research \& Development, 16(2), 188-200. doi:10.1017/S1463423614000243

Robben, S., Perry, M., Van Nieuwenhuijzen, L., Van Achterberg, T., Olde Rikkert, M., Schers, H., ... Melis, R. (2012). Impact of interprofessional education on collaboration attitudes, skills, and behavior among primary care professionals. Journal of Continuing Education in the Health Professions, 32(3), 196-204. doi:10.1002/chp.21145 
Roberge, P., Marchand, A., Reinharz, D., Marchand, L., \& Cloutier, K. (2004). Évaluation économique de la thérapie cognitivocomportementale des troubles anxieux. Canadian Psychology, 45, 202-218. doi: 10.1037/h0086988

Roberge, P., Fournier, L., Duhoux, A., Nguyen, C. T., \& Smolders, M. (2011). Mental health service use and treatment adequacy for anxiety disorders in Canada. Social Psychiatry and Psychiatric Epidemiology, 46(4), 321-330. doi:10.1007/s00127-010-0186-2

Roberge, P., Fournier, L., Menear, M., \& Duhoux, A. (2014). Access to psychotherapy for primary care patients with anxiety disorders. Canadian Psychology/Psychologie Canadienne, 55(2), 60-67. doi:10.1037/a0036317

Roca, M., Gili, M., Garcia-Garcia, M., Salva, J., Vives, M., Garcia Campayo, J., \& Comas, A. (2009). Prevalence and comorbidity of common mental disorders in primary care. Journal of Affective Disorders, 119(1), 52-58. doi:10.1016/j.jad.2009.03.014

Rodolfa, E., Bent, R., Eisman, E., Nelson, P., Rehm, L., \& Ritchie, P. (2005). A cube model for competency development: Implications for psychology educators and regulators. Professional Psychology: Research and Practice, 36(4), 347-354. doi:10.1037/0735-7028.36.4.347

Rodriguez, B. F., Weisberg, R. B., Pagano, M. E., Machan, J. T., Culpepper, L., \& Keller, M. B. (2004). Frequency and patterns of psychiatric comorbidity in a sample of primary care patients with anxiety disorders. Comprehensive Psychiatry, 45(2), 129-137. doi: 10.1016/j.comppsych.2003.09.005

Romanow, R. J., \& Marchildon, G. P. (2003). Psychological services and the future of health care in Canada. Canadian Psychology, 44, 283-295. doi: 10.1037/h0086954

Rosenfield, P. L. (1992). The potential of transdisciplinary research for sustaining and extending 
linkages between the health and social sciences. Social Science \& Medicine, 35(11), 13431357. doi:10.1016/0277-9536(92)90038-R

Rosser, W. W., Colwill, J. M., Kasperski, J., \& Wilson, L. (2011). Progress of Ontario's family health team model: A patient-centered medical home. Annals of Family Medicine, 9(2), 165-171. doi:10.1370/afm.1228

Rouse, J. (2011). Examining the effectiveness of psychotherapy provided at a Canadian university counselling centre: Preliminary results (Unpublished master's thesis). Ryerson University, Toronto, ON.

Rouse, J., McShane, K., \& Monson, C. (2012). Providing psychological services and training in primary care: Ryerson University and St. Michael's Hospital. Psynopsis 34(4), 24.

Roy-Byrne, P., Craske, M. G., Sullivan, G., Rose, R. D., Edlund, M. J., Lang, A. J., ... Stein, M. B. (2010). Delivery of evidence-based treatment for multiple anxiety disorders in primary care: A randomized controlled trial. JAMA, 303(19), 1921-1928. doi:10.1001/jama.2010.608

Sargeant, J., Loney, E., \& Murphy, G. (2008). Effective interprofessional teams: “Contact is not enough" to build a team. Journal of Continuing Education in the Health Professions, 28 (4), 228-234. doi:10.1002/chp.189

Schulte, T. J., Isley, E., Link, N., Shealy, C. N., \& Winfrey, L. L. (2004). General practice, primary care, and health service psychology: Concepts, competencies, and the combinedintegrated model. Journal of Clinical Psychology, 60, 1011-1025. doi: $10.1002 /$ jclp.20032

Seamless Care. (2008). Final Report: June 1, 2005 to March 31, 2008. Seamless care: An interprofessional education project for innovative team-based transition care. Retrieved 
from_https://www.cihc.ca/library/bitstream/10296/188/1/NS_SeamlessCare_FinalReport _2008.pdf

Seekles, W., Cuijpers, P., Kok, R., Beekman, A., van Marwijk, H., \& van Straten, A. (2013).

Psychological treatment of anxiety in primary care: A meta-analysis. Psychological Medicine, 43(2), 1-11. doi:10.1017/S0033291712000670

Seligman, M. E. P. (1995). The effectiveness of psychotherapy: The Consumer Reports study. American Psychologist, 50, 965-974. doi: 10.1037/0003-066X.50.12.965

Sharp, D., \& Morell, D. (1989). The psychiatry of general practice. In P. Williams, G.

Wilkinson, \& K. Rawnsley (Eds.), Scientific approaches to epidemiological psychiatry. London, England: Routledge.

Simon, G. (1992). Psychiatric disorder and functional somatic symptoms as predictors of health care use. Psychiatric Medicine, 10(3), 49-60.

Singer, J. D., \& Willett, J. B. (2009). Applied longitudinal data analysis: Modeling change and event occurrence. New York, NY: Oxford Scholarship Online. doi: 10.1093/acprof:oso/9780195152968.001.0001

Sladen, B. (1979). Health care psychology and graduate education. Professional Psychology, 10, 841-851. doi: 10.1037/0735-7028.10.6.841

Slater, M., Nicholas, E., Leung, F., \& Lofters, A. (2016). Provider perceptions of knowledge exchange and communication in a multisite family health team. Canadian Family Physician, 62 (5), 415. Retrieved from http://www.cfp.ca/content/62/5/415.abstract

Smetanin, P., Stiff, D., Briante, C., Adair, C., Ahmad, S. \& Khan, M. (2011). The life and economic impact of major mental illnesses in Canada: 2011 to 2041. Risk Analytica, on behalf of the Mental Health Commission of Canada. 
Smith, M. L., \& Glass, G.V. (1977). Meta-analysis of psychotherapy outcomes studies. American Psychologist, 32, 752-760. Retrieved from http://colorado.academia.edu/GeneGlass/Papers/234147/MetaAnalysis_of_Psychotherapy_Outcome_Studies

Soklaridis, S., Oandasan, I., \& Kimpton, S. (2007). Family health teams: Can health professionals learn to work together? Canadian Family Physician Médecin De Famille Canadien, 53, 1198-1199. Retrieved from http://www.cfp.ca/content/53/7/1198.full.pdf+html

Statistics Canada. (2003). Canadian community health survey: Mental health and well-being. The Daily, 3 September. Retrieved from http:// www.statcan.gc.ca/daily-quotidien/030903/ dq030903a-eng.htm.nu.ca/PDF/Suicide\%20Prevention\%20Strategy_final.pdf.

Stephens, T., \& Joubert, N. (2001). The economic burden of mental health problems in Canada. Chronic Diseases in Canada, 22, 18-23. Retrieved from http://www.phacaspc.gc.ca/publicat/cdic-mcc/22-1/d_e.html

Storrie, K., Ahern, K., \& Tuckett, A. (2010). A systematic review: Students with mental health problems--a growing problem. International Journal of Nursing Practice, 16(1), 1-6. doi:10.1111/j.1440-172X.2009.01813.x

Swenson, J. R., Aubrey, T., Gillis, K., MacPhee, C., Busing, N., Kates, ... Runnels, V. (2008). Development and implementation of a collaborative mental health care program in a primary care setting: The Ottawa Share Program. Canadian Journal of Community Mental Health, 27, 75-91.

Talen, M. R., Fraser, J. S., \& Cauley, K. (2005). Training primary care psychologists: A model for predoctoral programs. Professional Psychology: Research and Practice, 36, 136-143. 
doi: $10.1037 / 0735-7028.36 .2 .136$

Titov, N., Andrews, G., Johnston, L., Robinson, E., \& Spence, J. (2010). Transdiagnostic internet treatment for anxiety disorders: A randomized controlled trial. Behaviour Research and Therapy, 48(9), 890-899. doi:10.1016/j.brat.2010.05.014

Tracey, T. J., \& Kokotovic, A. M. (1989). Factor structure of the working alliance inventory. Psychological Assessment: A Journal of Consulting and Clinical Psychology, 1, 207-210. doi: 10.1037/1040-3590.1.3.207

Troeung, L., Egan, S. J., \& Gasson, N. (2014). A waitlist-controlled trial of group cognitive behavioural therapy for depression and anxiety in Parkinson's disease. $B M C$ Psychiatry, 14(1), 19-19. doi:10.1186/1471-244X-14-19

Tsang, E. S., Cheung, C. C., \& Sakakibara, T. (2016). Perceptions of interprofessionalism in health professional students participating in a novel community service initiative. Journal of Interprofessional Care, 30(1), 132. doi: 10.3109/13561820.2015.1055717

Turcotte, V. (2005). L'intégration de psychologues dans des équipes multidisciplinaires de première ligne: Facteurs facilitant et obstacles. (Doctoral dissertation, Université Laval). Retrieved from http://theses.ulaval.ca/archimede/fichiers/23243/23243.html

Twilling, L. L., Sockell, M. E., \& Sommers, L. S. (2000). Collaborative practice in primary care: Integrated training for psychologists and physicians. Professional Psychology: Research and Practice, 31, 685-691. doi: 10.1037/0735-7028.31.6.685

Umphress, V. J., Lambert, M. J., Smart, D. W., Barlow, S. H., \& Clouse, G. (1997). Concurrent and construct validity of the Outcome Questionnaire. Journal of Psychoeducational Assessment, 15, 40-55. doi: 10.1177/073428299701500104

United Kingdom Department of Health. (2001). Treatment choice in psychological therapies and 
counselling: Evidence based clinical practice guidelines. Retrieved from http://www.dh.gov.uk/prod_consum_dh/groups/dh_digitalassets/@dh/@en/documents/di gitalasset/dh_4058245.pdf

Valentine, M. A., Nembhard, I. M., \& Edmondson, A. C. (2015). Measuring teamwork in health care settings: A review of survey instruments. Medical Care, 53(4), e16-e30. doi: 10.1097/MLR.0b013e31827feef6

Vallis, T. M., Shaw, B. F., \& Dobson, K. S. (1986). The cognitive therapy scale: Psychometric properties. Journal of Consulting and Clinical Psychology, 54(3), 381-385. doi:10.1037//0022-006X.54.3.381

Vari, P. M., Lash, J., Brown, S. S., Porter, A. G., Trinkle, D., Garber, J. S., ... Vari, R. C. (2013). Collaborative practice education: The effect of an interprofessional teamwork course on students' knowledge and skills. Medical Science Educator, 23(S3), 494-501. doi:10.1007/BF03341672

Vasiliadis, H.M., Lesage, A., Adair, C., \& Boyer, R. (2005). Service use for mental health reasons: Cross-provincial differences in rates, determinants, and equity of access. Canadian Journal of Psychiatry, 50, 614-619. Retrieved from http://ww1.cpaapc.org:8080/Publications/Archives/CJP/2005/september/cjp-sept-05-vasiliadis-7.pdf

Vasiliadis, H. M., Tempier, R., Lesage, A., \& Kates, N. (2009). General practice and mental health determinants of outpatient service use. Canadian Journal of Psychiatry, 54, 468475. Retrieved from http://findarticles.com/p/articles/mi_7486/is_20090701/ai_n32433908/

Vaughan, B., Macfarlane, C., Dentry, T., \& Mendoza, G. (2014). The interdisciplinary education perception scale (IEPS): Which factor structure? Education in Medicine Journal, 6(3) 
doi:10.5959/eimj.v6i3.259

Vermeersch, D. A., Lambert, M. J., \& Burlingame, G. M. (2000). Outcome Questionnaire: Item sensitivity to change. Journal of Personality Assessment, 74, 242-261. doi: 10.1207/S15327752JPA7402_6

Vermeersch, D. A., Whipple, J. L., Hawkins, E. J., Burchfield, C. M., \& Okiishi, J. C. (2004). Outcome Questionnaire: Is it sensitive to changes in counseling center clients? Journal of Counseling Psychology, 51, 38-49. doi: 10.1037/0022-0167.51.1.38

Vickers, K. S., Ridgeway, J. L., Hathaway, J. C., Egginton, J. S., Kaderlik, A. B., \& Katzelnick, D. J. (2013). Integration of mental health resources in a primary care setting leads to increased provider satisfaction and patient access. General Hospital Psychiatry, 35(5), 461-467. doi:10.1016/j.genhosppsych.2013.06.011

Wang, P. S., Berglund, P. A., Olfson, M., \& Kessler, R. C. (2004). Delays in initial treatment contact after first onset of a mental disorder. Health Services Research, 39, 393-416. doi: 10.1111/j.1475-6773.2004.00234.x

Wang, P. S., Lane, M., Olfson, M., Pincus, H. A., Wells, K. B., \& Kessler, R. C. (2005). Twelvemonth use of mental health services in the United States. Results from the national comorbidity survey replication. Archives of General Psychiatry, 62, 629-640. Retrieved from http://archpsyc.amaassn.org/cgi/reprint/62/6/629? maxtoshow=\&hits=10\&RESULTFORMAT=\&fulltext=Tw elve-month + use + of + mental + health

Ware, J.E., Jr., Kosinski, M., Turner-Bowker, D.M., Gandek, B. (2002). How to score version 2 of the SF-12v2® health survey (with a supplement documenting SF-12® health survey) Lincoln, RI: Quality Metric Incorporated. 
Webb, C. A., DeRubeis, R. J., Amsterdam, J. D., Shelton, R. C., Hollon, S. D., \& Dimidjian, S. (2011). Two aspects of the therapeutic alliance: Differential relations with depressive symptom change. Journal of Consulting and Clinical Psychology, 79(3), 279-283. doi:10.1037/a0023252

Wener, P., \& Woodgate, R. L. (2016). Collaborating in the context of co-location: A grounded theory study. BMC Family Practice, 17, 30. doi: 10.1186/s12875-016-0427-x

White, J. (2008). CBT and the challenge of primary care: Developing effective, efficient, equitable, acceptable and accessible services for common mental health problems. Journal of Public Mental Health, 7, 32-41. doi: 10.1108/17465729200800006

Singer, J. D., \& Willett, J. B. (2003). Applied longitudinal data analysis: Modeling change and event occurrence. New York, NY: Oxford University Press.

Woodall, A., Morgan, C., Sloan, C., \& Howard, L. (2010). Barriers to participation in mental health research: Are there specific gender, ethnicity and age related barriers? BioMed Central Psychiatry, 10(1), 103-103. doi:10.1186/1471-244X-10-103

World Health Organization. (2007). What is mental health? Retrieved from http://www.who.int/features/qa/62/en/index.html

World Health Organization. (2016). Enhancing primary healthcare deliver in the inner city in Toronto, Canada. Retrieved from http://www.integratedcare4people.org/practices/231/enhancing-primary-healthcaredelivery-in-the-inner-city-community-in-torontocanada/?utm_source $=$ mailoutinteractive \&utm_medium $=$ email\&utm_campaign $=$ Bringing $\%$ 20LGBTQ\%20history\%20out\%20of\%20the\%20dark

Xyrichis, A., \& Lowton, K. (2008). What fosters or prevents interprofessional teamworking in 
primary and community care? A literature review. International Journal of Nursing Studies, 45, 140-153. doi: 10.1016/j.ijnurstu.2007.01.015

Yong, A. G., \& Pearce, S. (2013). A beginners guide to factor analysis: Focusing on exploratory factor analysis. Tutorials in Quantitative Methods for Psychology, 9(2), 79-94.

Young, J. E., \& Beck, A.T. (1980). Cognitive therapy scale. Bala Cynwyd, PA: Beck Institute for Cognitive Behavior Therapy.

Zwick, W. R., \& Velicer, W. F. (1986). Comparison of five rules for determining the number of components to retain. Psychological Bulletin, 99(3), 432-442. doi:10.1037/00332909.99.3.432 